\title{
ON CONGRUENCE MODULES RELATED TO HILBERT EISENSTEIN SERIES
}

\author{
by \\ Sheng-Chi Shih \\ Copyright (c) Sheng-Chi Shih 2018 \\ A Dissertation Submitted to the Faculty of the \\ DEPARTMENT OF MATHEMATICS \\ In Partial Fulfillment of the Requirements \\ For the Degree of \\ DOCTOR OF PHILOSOPHY \\ In the Graduate College \\ THE UNIVERSITY OF ARIZONA
}




\section{THE UNIVERSITY OF ARIZONA \\ GRADUATE COLLEGE}

As members of the Dissertation Committee, we certify that we have read the dissertation prepared by Sheng-Chi Shih entitled On congruence modules related to Hilbert Eisenstein series and recommend that it be accepted as fulfilling the dissertation requirement for the Degree of Doctor of Philosophy.

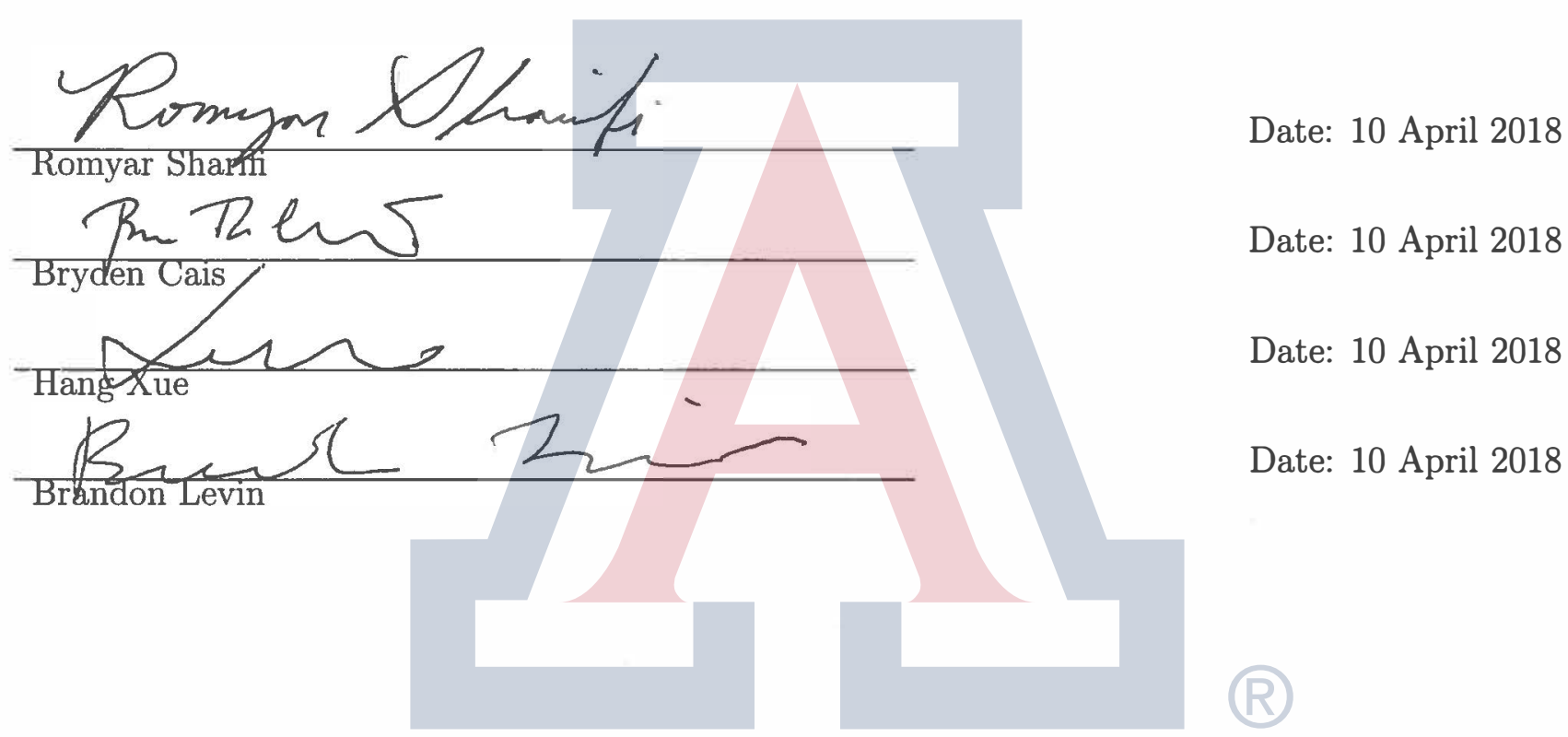

Final approval and acceptance of this dissertation is contingent upon the candidate's submission of the final copies of the dissertation to the Graduate College.

I hereby certify that I have read this dissertation prepared under my direction and recommend that it be accepted as fulfilling the dissertation requirement.

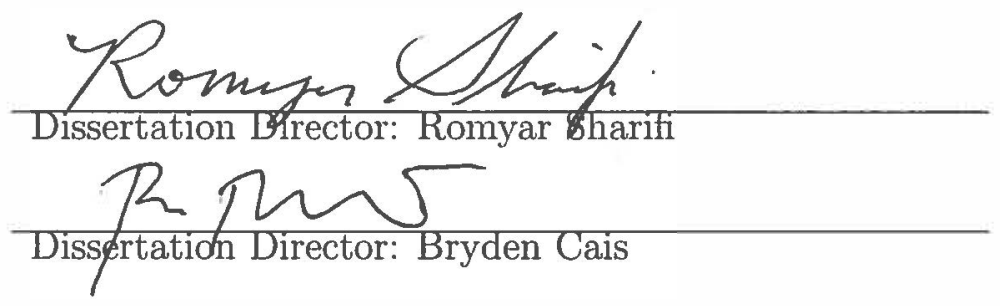

Date: 10 April 2018

Date: 10 April 2018 


\section{STATEMENT BY AUTHOR}

This dissertation has been submitted in partial fulfillment of requirements for an advanced degree at the University of Arizona and is deposited in the University Library to be made available to borrowers under rules of the Library.

Brief quotations from this dissertation are allowable without special permission, provided that accurate acknowledgment of source is made. Requests for permission for extended quotation from or reproduction of this manuscript in whole or in part may be granted by the head of the major department or the Dean of the Graduate College when in his or her judgment the proposed use of the material is in the interests of scholarship. In all other instances, however, permission must be obtained from the author. 


\section{ACKNOWLEDGEMENTS}

First and foremost, I would like to express my gratitude to my advisor, Romyar Sharifi, for the support of my research, for his patience, and for all of his guidance. Also, I would like to thank my committee members: Bryden Cais, Brandon Levin, and Hang Xue for their suggestions on my work and their comments on the earlier version of my thesis.

I would like to thank my friends in the Department of Mathematics at the University of Arizona: Ding Ma, Jun Wang, Lanbo Fang, Yuan Tao, Jinjin Liang, Liang Wu, Miao Zhang, Cody Lee Gunton, for the discussions in the past six years.

Last but not the least, I would like to thank my family: my parents Kuo-Chen Shih, Jin-Yin Lu, and my wife Chia-Yun Tsai for their support throughout my life. 


\section{DEDICATION}

For my wife, Chia-Yun Tsai 
TABLE OF CONTENTS

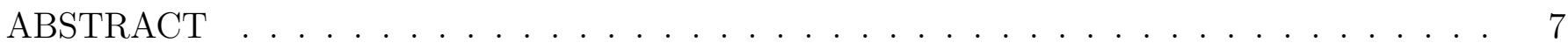

CHAPTER 1 Introduction . . . . . . . . . . . . . . . . . . . . 8

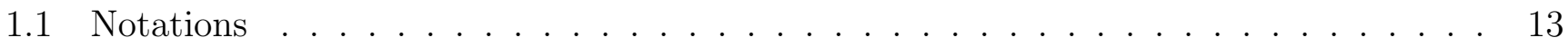

CHAPTER 2 Preliminaries . . . . . . . . . . . . . . . . . . . . 14

2.1 Grossencharacters and Hecke characters . . . . . . . . . . . . . . . . . . . 14

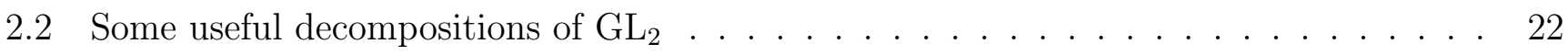

2.3 Local integrals . . . . . . . . . . . . . . . . . . . . . . . . . . . . 24

2.4 Local epsilon factors . . . . . . . . . . . . . . . . . . . . . . . . . . . . . . . . . . . . . . . . . . . . . . . . . . . .

2.5 Character sums . . . . . . . . . . . . . . . . . . . . 29

CHAPTER 3 Hilbert modular forms . . . . . . . . . . . . . . . . . . . . . . 34

3.1 Classical Hilbert modular forms . . . . . . . . . . . . . . . . . . . . . . . . . . 34

3.2 Adelic Hilbert modular forms . . . . . . . . . . . . . . . . . . . . . . . . 37

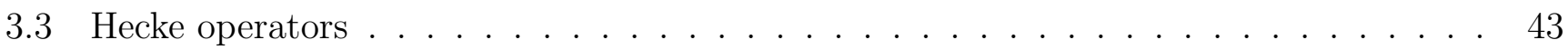

3.4 Cusps of Hilbert modular varieties . . . . . . . . . . . . . . . . . . . . 47

CHAPTER 4 Automorphic forms . . . . . . . . . . . . . . . . . . . . 58

4.1 Non-archimedean sections . . . . . . . . . . . . . . . . . . . . . . . 58

4.2 Archimedean sections . . . . . . . . . . . . . . . . . . 76

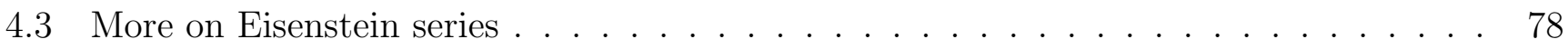

4.4 More on the constant terms of Eisenstein series . . . . . . . . . . . . . . 87

CHAPTER $5 \quad \Lambda$-adic modular forms . . . . . . . . . . . . . . . . . . . . 91

5.1 Definitions . . . . . . . . . . . . . . . . . . . . . . . 91

$5.2 \quad \Lambda$-adic Eisenstein series . . . . . . . . . . . . . . . . . . . . . . . . . . . . . . . . . . . . . . 94

5.3 Control Theorem . . . . . . . . . . . . . . . . . . . . . 99

CHAPTER 6 Hilbert modular varieties . . . . . . . . . . . . . . . . . . . . 102

6.1 Moduli problems with level structures . . . . . . . . . . . . . . . . . . 102

6.2 Geometric modular forms . . . . . . . . . . . . . . . . . . . 106

6.3 Toroidal compactification . . . . . . . . . . . . . . . . . . 111

6.4 Minimal compactification . . . . . . . . . . . . . . . . . . . . 111

6.5 p-adic modular forms . . . . . . . . . . . . . . . . . . . . . . . 114

CHAPTER 7 Main results . . . . . . . . . . . . . . . . . . . . 123

7.1 Proof of Theorem 1.0.1: Part 1 . . . . . . . . . . . . . . . . . . 123

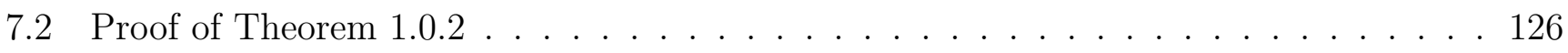

7.3 Proof of Theorem 1.0.1: Part $2 \ldots \ldots \ldots \ldots$. . . . . . . . . . . 132

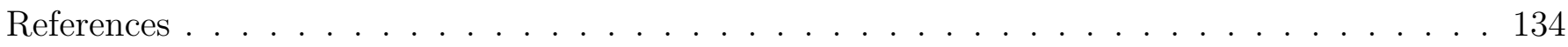




\section{ABSTRACT}

We generalize the work of Ohta on the congruence modules attached to elliptic Eisenstein series to the setting of Hilbert modular forms. Our work involves three parts. In the first part, we construct Eisenstein series adelically and compute their constant terms by computing local integrals. In the second part, we prove a control theorem for one-variable ordinary $\Lambda$-adic modular forms following Hida's work on the space of multivariable ordinary $\Lambda$-adic cusp forms. In part three, we compute congruence modules related to Hilbert Eisenstein series through an analogue of Ohta's methods. 


\title{
CHAPTER 1
}

\author{
Introduction
}

Let $R$ be an integral domain with quotient field $Q(R)$. We consider a short exact sequence of flat $R$-modules

$$
0 \longrightarrow A \stackrel{i}{\longrightarrow} B \stackrel{p}{\longrightarrow} C \longrightarrow 0
$$

Suppose this short exact sequence splits after tensoring with $Q(R)$ over $R$, i.e., we have

$$
0 \longleftarrow A \otimes_{R} Q(R) \leftarrow^{t} B \otimes_{R} Q(R) \leftarrow^{s} C \otimes_{R} Q(R) \longleftarrow 0 .
$$

The congruence module attached to these data is defined by

$$
\mathcal{C}:=C / p(B \cap s(C))
$$

Congruence modules have been studied by many people in different settings. For instance, Harder and Pink $[\mathrm{H}-\mathrm{P}]$ considered the following short exact sequence of $\mathbb{Z}_{p}$-modules

$$
0 \longrightarrow H_{P}^{1}\left(\Gamma, \mathbb{Z}_{p}\right)_{E} \longrightarrow H^{1}\left(\Gamma, \mathbb{Z}_{p}\right)_{E} \longrightarrow \mathbb{Z}_{p} \longrightarrow 0
$$

where $H_{P}^{1}$ is the first parabolic cohomology, the subscript "E" means the Eisenstein component, and $\Gamma$ is a congruence subgroup of $\mathrm{SL}_{2}(\mathbb{Z})$. In [Ohta4], Ohta computed the congruence module associated with the sequence

$$
0 \longrightarrow S^{\text {ord }}(\Gamma ; \Lambda)_{E} \longrightarrow M^{\text {ord }}(\Gamma ; \Lambda)_{E} \stackrel{\text { res }}{\longrightarrow} \Lambda \longrightarrow 0
$$

where res is the residue map, and $M^{\text {ord }}(\Gamma ; \Lambda)$ and $S^{\text {ord }}(\Gamma ; \Lambda)$ are respectively the spaces of ordinary $\Lambda$ adic modular forms and ordinary $\Lambda$-adic cusp forms, where $\Lambda=\mathfrak{o}[|T|]$ for some extension $\mathfrak{o}$ of $\mathbb{Z}_{p}$. In 
this paper, we generalize Ohta's work to the setting of Hilbert modular forms. For the above examples and our main result (Theorem 1.0.1), we require that splittings are Hecke equivariant. Moreover, there exist canonical splittings which are considered in the computation of these congruence modules.

Before we describe our main results, let us mention our motivation, which comes from Sharifi's conjecture [Sha]. Sharifi's conjecture is a refinement of the Iwasawa main conjecture. The main conjecture asserts a relationship between two objects: one is a certain $p$-adic $L$-function and the other is a characteristic polynomial associated with the $p$-part of the class group of the cyclotomic $\mathbb{Z}_{p}$-extension of an abelian extension of $\mathbb{Q}$. Roughly, Sharifi's conjecture predicts that one can obtain the information on the second object from the geometry of modular curves. The main conjecture over $\mathbb{Q}$ was first proved by Mazur and Wiles $[\mathrm{MW}]$ using 2-dimensional Galois representations attached to cusp forms that are congruent to ordinary Eisenstein series. Wiles [Wil3] generalized the method of Mazur-Wiles to the setting of Hilbert modular forms and proved the main conjecture over totally real fields. Combining his previous works [Ohta2] and [Ohta3], Ohta gave a refinement of Mazur-Wiles proof of the main conjecture over $\mathbb{Q}$ examining the action of $\operatorname{Gal}(\overline{\mathbb{Q}} / \mathbb{Q})$ on the Eisenstein component of the cohomology of modular curves. The cohomology of modular curves provides a canonical choice of a lattice in a Galois representation, which plays an important role in Sharifi's work. Our work is a first step to proving the main conjecture over totally real fields along the lines of Ohta's approach and to generalizing Sharifi's conjecture to totally real fields.

To describe our results, we fix some notations first. Let $F$ be a totally real field, and let $\mathcal{O}_{F}$ be its ring of integers. We denote by $\mathfrak{D}$ the different of $F$. We fix an integral ideal $\mathfrak{n}$ of $F$ and an odd rational prime $p$ satisfying the following conditions

H1 $p$ does not divide $N_{F / \mathbb{Q}}(\mathfrak{n} \mathfrak{D}) \phi\left(N_{F / \mathbb{Q}}(\mathfrak{n})\right) h_{F}$, 
$\mathbf{H} \mathbf{2} \mathfrak{n}$ is prime to $\mathfrak{D}$ and does not divide 2 and 3 .

Here $\phi$ is the Euler phi-function, $h_{F}$ is the class number of $F$, and $N_{F / \mathbb{Q}}$ is the norm map from $F$ to $\mathbb{Q}$. Let $\chi_{1}$ and $\chi_{2}$ be primitive narrow ray class characters of conductor $\mathfrak{n}_{1}$ and $\mathfrak{n}_{2}$, respectively. We assume that $\mathfrak{n}_{1} \mathfrak{n}_{2}=\mathfrak{n}$ or $\mathfrak{n} p$, and $\mathfrak{n}_{2}$ is prime to $p$. Let $\Lambda=\mathbb{Z}_{p}\left[\chi_{1}, \chi_{2}\right][|T|]$. We denote by $\mathfrak{M}=\mathfrak{M}\left(\chi_{1}, \chi_{2}\right)\left(\right.$ resp. $\left.\mathfrak{m}=\mathfrak{m}\left(\chi_{1}, \chi_{2}\right)\right)$ the maximal ideal of the Hecke algebra $\mathcal{H}(\mathfrak{n} ; \Lambda)$ (resp. the cuspidal Hecke algebra $h(\mathfrak{n} ; \Lambda))$ containing the Eisenstein ideal $\mathcal{I}\left(\chi_{1}, \chi_{2}\right)\left(\operatorname{resp} . I\left(\chi_{1}, \chi_{2}\right)\right)$ associated to the Eisenstein series $\mathcal{E}\left(\chi_{1}, \chi_{2}\right)$ (see Chapter 5 and Chapter 7 for their definitions). We denote by $M^{\text {ord }}\left(\mathfrak{n}, \chi_{1} \chi_{2} ; \Lambda\right)\left(\right.$ resp. $\left.S^{\text {ord }}\left(\mathfrak{n}, \chi_{1} \chi_{2} ; \Lambda\right)\right)$ the space of $p$-ordinary $\Lambda$-adic modular forms (resp. cusp forms $)$ and denote by $M^{\text {ord }}\left(\mathfrak{n}, \chi_{1} \chi_{2} ; \Lambda\right)_{\mathfrak{M}}\left(\right.$ resp. $\left.S^{\text {ord }}\left(\mathfrak{n}, \chi_{1} \chi_{2} ; \Lambda\right)_{\mathfrak{M}}\right)$ the localization of $M^{\text {ord }}\left(\mathfrak{n}, \chi_{1} \chi_{2} ; \Lambda\right)$ (resp. $\left.S^{\text {ord }}\left(\mathfrak{n}, \chi_{1} \chi_{2} ; \Lambda\right)\right)$ at $\mathfrak{M}$ (see Chapter 4 for the definition of the above spaces). Let $\omega$ be the Teichmüller character. We extend it to a narrow ray class character modulo $p$, also denoted by $\omega$, defined by sending each prime to $p$ integral ideal $\mathfrak{a}$ of $F$ to $\omega\left(N_{F / \mathbb{Q}}(\mathfrak{a})\right)$. The following theorem is the first main result in this article.

Theorem 1.0.1. Assume that $\left(\chi_{1}, \chi_{2}\right) \neq\left(\omega^{-2}, \mathbf{1}\right)$ and $\chi_{1} \chi_{2}^{-1} \omega(\mathfrak{p}) \neq 1$ for some prime ideal $\mathfrak{p} \mid p$. Then the congruence modules attached to the short exact sequences of $\Lambda$-modules

$$
\left\{\begin{array}{l}
0 \longrightarrow S^{\text {ord }}\left(\mathfrak{n}, \chi_{1} \chi_{2} ; \Lambda\right)_{\mathfrak{M}} \longrightarrow M^{\text {ord }}\left(\mathfrak{n}, \chi_{1} \chi_{2} ; \Lambda\right)_{\mathfrak{M}} \stackrel{C_{0}}{\longrightarrow} \Lambda \longrightarrow 0 \\
0 \longrightarrow \mathcal{I}\left(\chi_{1}, \chi_{2}\right) \longrightarrow \mathcal{H}^{\text {ord }}(\mathfrak{n} ; \Lambda)_{\mathfrak{M}} \longrightarrow \Lambda \longrightarrow 0
\end{array}\right.
$$

are both $\Lambda /\left(A\left(\chi_{1}, \chi_{2}\right)\right)$, where $A\left(\chi_{1}, \chi_{2}\right) \in \Lambda$ is a Deligne-Ribet p-adic L-function times an element in $\Lambda$, and the map $C_{0}$ maps each modular form to a formal sum of its constant terms at cusps.

The map $C_{0}$ will be defined in Section 3.4, and the element $A\left(\chi_{1}, \chi_{2}\right)$ is related to the constant terms of the Eisenstein series $\mathcal{E}\left(\chi_{1}, \chi_{2}\right)$, which will be defined in Section 7.1. When $F=\mathbb{Q}$, Theorem 1.0.1 was proved by Ohta [Ohta4]. There are couple of difficulties in the setting of Hilbert 
modular forms. For example, the residue map does not exist, and the class number $h_{F}$ of $F$ is not 1 in general. To overcome those difficulties, we will describe everything adelically, including the space of modular forms and the set of cusps.

From the first short exact sequence in (1.1), we obtain a $\Lambda$-adic cusp form $F_{S}$ which is congruent to the Eisenstein series $\mathcal{E}\left(\chi_{1}, \chi_{2}\right)$ modulo $A\left(\chi_{1}, \chi_{2}\right)$. One can associate to $F_{S}$ a surjective $\Lambda$-module homomorphism

$$
\Psi: h^{\text {ord }}(\mathfrak{n} ; \Lambda)_{\mathfrak{M}} / I\left(\chi_{1}, \chi_{2}\right) \rightarrow \Lambda /\left(A\left(\chi_{1}, \chi_{2}\right)\right) ; T \mapsto C\left(1, T \cdot F_{S}\right)
$$

where $C\left(1, T \cdot F_{S}\right)$ is the first Fourier coefficient of $T \cdot F_{S}$ defined in Chapter 5 . The following is the second main result in this paper.

Theorem 1.0.2. Let the notation be as above. Then we have an isomorphism of $\Lambda$-modules

$$
\Psi: h^{\text {ord }}(\mathfrak{n} ; \Lambda)_{\mathfrak{M}} / I\left(\chi_{1}, \chi_{2}\right) \simeq \Lambda /\left(A\left(\chi_{1}, \chi_{2}\right)\right)
$$

When $F=\mathbb{Q}$, Ohta [Ohta4] proved Theorem 1.0.2 using the Iwasawa main conjecture. We borrow Emerton's idea [E] to give another proof without using the main conjecture. The idea is as follows. To show the injectivity, it suffices to show the existence of a Hecke operator $H \in \mathcal{H}$ such that for each $F \in M^{\text {ord }}\left(\mathfrak{n}, \chi_{1} \chi_{2} ; \Lambda\right)_{\mathfrak{M}}$, we have $C(1, H \cdot F)=C_{\lambda}(0, F)$ for all $\lambda=1, \ldots, h_{F}^{+}$, where $C_{\lambda}(0, F)$ are the constant terms of $F$ defined in Chapter 5. We will give an explicit construction of the Hecke operator $H$ in Chapter 7 . Here $h_{F}^{+}$is the narrow class number of $F$. Note that for all $F \in M^{\text {ord }}\left(\mathfrak{n}, \chi_{1} \chi_{2} ; \Lambda\right)_{\mathfrak{M}}$, we have $C_{1}(0, F)=\ldots=C_{h_{F}^{+}}(0, F)$, so the Hecke operator $H$ does not depend on $\lambda$.

We now give an outline of the thesis. In Chapter 2, we review some background: narrow ray class characters, decomposition of $\mathrm{GL}_{2}$, and local epsilon factors. Also, we write local epsilon characters as character sums, which will be used to prove Theorem 1.0.1. 
In Chapter 3, we review definitions and properties of Hilbert modular forms in both the classical and the adelic settings. Also, we formulate cusps in the adelic language, which plays an important role in stating Theorem 1.0.1.

In Chapter 4, we construct Eisenstein series adelically and compute their constant terms at different cusps. Indeed, one can do this in the classical setting (see [Ohta4] when $F=\mathbb{Q}$ and $[\mathrm{Oz}]$ when $F \neq \mathbb{Q}$ ). One reason we have to do everything adelically is to show that the map $C_{0}$ in Theorem 1.0.1 commutes with Hecke operators, which we can only prove in the adelic setting. In addition, it is difficult to write adelic cusps in the classical setting explicitly since to do so, one has to use the strong approximation for $\mathrm{GL}_{2}$. The way to construct Eisenstein series adelically is to choose certain local induced representations at each place of $F$. We then compute their constant terms by computing local integrals at all places of $F$. In principle, one can obtain the Fourier expansion at all cusps if one can compute all local integrals explicitly. This construction is well-known to experts and has been used to study the arithmetic of Eisenstein series for different algebraic groups such as unitary and symplectic groups (see [Hsieh] for example).

In Chapter 5 , we recall the definition of $\Lambda$-adic modular forms and construct $\Lambda$-adic Eisenstein series as examples. Also, we compute their constant terms at different cusps using results in Chapter 4, which will be used in the proof of Theorem 1.0.1.

In Chapter 6, our goal is to prove a control theorem (Corollary 6.5.5). When $F=\mathbb{Q}$, it was proved by Hida $[\mathrm{H} 2]$. When $F \neq \mathbb{Q}$, one can mimic his idea to deal with the case of one-variable ordinary $\Lambda$-adic Hilbert modular forms under the assumption of Leopoldt's conjecture. Without assuming Leopoldt's conjecture, Wiles [Wil2] proved a control theorem for the space of one-variable ordinary $\Lambda$-adic cusp forms, and Hida [H3] proved a theorem for the space of multivariable ordinary $\Lambda$-adic cusp forms via a different approach. We follow Hida's argument to prove a theorem for the space of 
one-variable ordinary $\Lambda$-adic Hilbert modular forms. This seems to be known to experts; however, we have not found any mention of it in the literature.

In the chapter, we prove Theorem 1.0.1 and Theorem 1.0.2.

\subsection{Notations}

Throughout this paper, we fix a totally real field $F$ with $[F: \mathbb{Q}]=d \geq 1$, and we let $\mathcal{O}_{F}$ be the ring of integers of $F$. We write $\widehat{\mathcal{O}}_{F}=\mathcal{O}_{F} \otimes_{\mathbb{Z}} \widehat{\mathbb{Z}}$, where $\widehat{\mathbb{Z}}=\prod_{p<\infty} \mathbb{Z}_{p}$. We denote by $\mathfrak{D}$ the different of $F$ and $d_{F}=N(\mathfrak{D})$ the discriminant of $F$. Here $N=N_{F / \mathbb{Q}}$ is the norm map form $F$ to $\mathbb{Q}$. We denote by $h_{F}=\left|\mathrm{Cl}_{F}\right|$ (resp. $\left.h_{F}^{+}=\left|\mathrm{Cl}_{F}^{+}\right|\right)$the class number of $F$ (resp. the narrow class number of $F$ ), where $\mathrm{Cl}_{F}$ (resp. $\mathrm{Cl}_{F}^{+}$) is the ideal class group of $F$ (resp. narrow ideal class group).

We fix a set $I=\left\{\tau_{1}, \cdots, \tau_{d}\right\}$ of distinct real embeddings of $F$ into $\mathbb{R}$. For any element $f$ in $F$, by $f \gg 0$, we mean that $f$ is totally positive, i.e., $\tau_{i}(f)>0$ for all $i=1, \ldots, d$. For any subset $A$ of $F$, we denote by $A^{+}$the subset of totally positive elements in $A$, i.e., for any $f$ in $A, f \in A^{+}$if $f \gg 0$.

For each finite place $v$ of $F$, we denote by $F_{v}$ the completion of $F$ at $v, \mathcal{O}_{v}$ its ring of integers, $\mathfrak{p}_{v}$ the maximal ideal of $\mathcal{O}_{\mathfrak{p}_{v}}$, and $\varpi_{\mathfrak{p}_{v}}$ a fixed uniformizer. We denote by $q_{v}$ the cardinality of the residue field $\mathcal{O}_{\mathfrak{p}_{v}} / \varpi_{\mathfrak{p}_{v}}$. Let $\operatorname{val}_{v}$ be the normalized valuation such that $\operatorname{val}_{v}\left(\varpi_{\mathfrak{p}_{v}}\right)=1$. Sometimes, we write $\varpi_{\mathfrak{p}_{v}}$ as $\varpi_{v}$, and $\operatorname{val}_{v}$ as $\operatorname{val}_{\mathfrak{p}_{v}}$ for simplicity. In addition, we will omit $v$ from $\mathfrak{p}_{v}, \varpi_{v}, q_{v}$, and $\operatorname{val}_{v}$ if there is no confusion.

Finally, we fix, once and for all, embeddings of $\overline{\mathbb{Q}}$ in $\overline{\mathbb{Q}_{p}}$ and in $\mathbb{C}_{p}$. 


\section{CHAPTER 2}

\section{Preliminaries}

\subsection{Grossencharacters and Hecke characters}

In this section, we review the definitions of grossencharacters, narrow ray class characters, Hecke characters, and their relationship. We only consider characters over totally real fields. The definition of characters over arbitrary number fields is very similar. One can see [Neu, VII, §6] for more information.

Let $\mathfrak{n}$ be an integral ideal of the totally real field $F$ and denote by $I_{\mathfrak{n}}$ the group of all fractional ideals that are relatively prime to $\mathfrak{n}$. For $a \in \mathcal{O}_{F}$, the congruence $a \equiv 1 \bmod \mathfrak{n} \operatorname{means} \operatorname{val}_{\mathfrak{p}}(a-1) \geq \operatorname{val}_{\mathfrak{p}}(\mathfrak{n})$ for all prime ideals $\mathfrak{p} \mid \mathfrak{n}$ or equivalently, $a-1 \in \mathfrak{n}$. We extend this congruence to the case of elements in $F^{\times}$. Namely, $a \equiv 1 \bmod \mathfrak{n}$ means there exist $b, c \in \mathcal{O}_{F}$ relatively prime to $\mathfrak{n}$ such that $a=\frac{b}{c}$ and $b \equiv c \bmod \mathfrak{n}$. Let

$$
\begin{gathered}
\mathcal{O}(\mathfrak{n})=\left\{a \in \mathcal{O}_{F} \mid((a), \mathfrak{n})=1\right\}, F(\mathfrak{n})=\left\{a \in F^{\times} \mid((a), \mathfrak{n})=1\right\}, \\
\mathcal{O}_{\mathfrak{n}}^{\times}=\left\{u \in \mathcal{O}_{F}^{\times} \mid u \equiv 1 \bmod \mathfrak{n}\right\}, F_{\mathfrak{n}}=\left\{u \in F^{\times} \mid u \equiv 1 \bmod \mathfrak{n}\right\} .
\end{gathered}
$$

Here $((a), \mathfrak{n})=1$ means $(a)+\mathfrak{n}=\mathcal{O}_{F}$. We denote by $P(\mathfrak{n})$ the group of principal fractional ideals (a) with $a \in F(\mathfrak{n})$. The following lemma is obvious.

Lemma 2.1.1. Let the notation be as above. For any nontrivial integral ideal $\mathfrak{n}$ of $F$, there is an isomorphism

$$
\phi:\left(\mathcal{O}_{F} / \mathfrak{n}\right)^{\times} \simeq F(\mathfrak{n}) / F_{\mathfrak{n}} ; a+\mathfrak{n} \mapsto a F_{\mathfrak{n}}
$$




\section{Grossencharacters}

Definition 2.1.2. Let $G$ be a topological group. A character of $G$ is a continuous homomorphism $\chi: G \longrightarrow \mathbb{C}^{\times}$

If the image of $\chi$ is contained in the unit circle $S^{1}$, one calls $\chi$ a unitary character. Some authors refer to characters as quasi-characters and reserve the term "characters" for unitary characters. For our purpose, we only consider characters with image in $S^{1}$, so we will call them characters instead of unitary characters for simplicity.

Note that the set of the characters of $\left(\mathcal{O}_{F} / \mathfrak{n}\right)^{\times}$are in one-to-one correspondence with the set of characters of $F(\mathfrak{n})$ which are trivial on $F_{\mathfrak{n}}$. Indeed, any $a \in F(\mathfrak{n})$ can be written as $\frac{b}{c}$ with $b, c \in \mathcal{O}(\mathfrak{n})$. Thus any character of $\left(\mathcal{O}_{F} / \mathfrak{n}\right)^{\times}$extends to $F(\mathfrak{n})$ by $\chi\left(\frac{b}{c}\right)=\chi(b) \chi(c)^{-1}$.

By writing $\mathfrak{n}=\prod_{\mathfrak{p} \mid \mathfrak{n}} \mathfrak{p}^{e_{\mathfrak{p}}}$, one can decompose each character $\chi$ of $\left(\mathcal{O}_{F} / \mathfrak{n}\right)^{\times}$as

$$
\chi=\prod_{\mathfrak{p} \mid \mathfrak{n}} \chi^{(\mathfrak{p})}: \prod_{\mathfrak{p} \mid \mathfrak{n}}\left(\mathcal{O}_{F} / \mathfrak{p}^{e_{\mathfrak{p}}}\right)^{\times} \longrightarrow S^{1} .
$$

We denote by $I_{\mathfrak{n}}$ the set of fractional ideals of $F$ which are prime to $\mathfrak{n}$. The definition of Grossencharacter is as follows.

Definition 2.1.3. A Grossencharacter $\bmod \mathfrak{n}$ is a character $\chi: I_{\mathfrak{n}} \longrightarrow S^{1}$ for which there exists a pair of characters

$$
\chi_{f}:\left(\mathcal{O}_{F} / \mathfrak{n}\right)^{\times} \longrightarrow S^{1}, \chi_{\infty}:\left(\mathbb{R}^{\times}\right)^{d} \longrightarrow S^{1}
$$

such that

$$
\chi((a))=\chi_{f}(a) \chi_{\infty}(a) \text { for all } a \in \mathcal{O}(\mathfrak{n})
$$

Here we evalute the character $\chi_{\infty}$ of $\left(\mathbb{R}^{\times}\right)^{d}$ on $\mathcal{O}(\mathfrak{n})$ by using the real embeddings of $F$, i.e., we have

$$
\chi_{\infty}(a)=\chi_{\infty}\left(\prod_{i=1}^{d} \tau_{i}(a)\right)
$$


where $\tau_{1}, \ldots, \tau_{d}$ are the distinct real embeddings of $F$ fixed in Chapter 1 .

Lemma 2.1.4. The Grossencharacter $\chi$ determines the pair $\left(\chi_{f}, \chi_{\infty}\right)$ uniquely. We call $\chi_{f}$ the finite part of $\chi$ and $\chi_{\infty}$ the infinite part of $\chi$. Conversely, a pair of characters $\chi_{f}:\left(\mathcal{O}_{F} / \mathfrak{n}\right)^{\times} \longrightarrow S^{1}$ and $\chi_{\infty}:\left(\mathbb{R}^{\times}\right)^{d} \longrightarrow S^{1}$ comes from a Grossencharacter mod $\mathfrak{n}$ if and only if $\chi_{f}(a) \chi_{\infty}(a)=1$ for all $a \in \mathcal{O}_{F}^{\times}$

Proof. For the first assertion, it suffices to show $\chi_{\infty}$ is uniquely determined by $\chi$. For any $a=\frac{b}{c} \in F_{\mathfrak{n}}$, we have $\chi_{f}(b)=\chi_{f}(c)$, which implies that $\chi_{\infty}(a)=\chi((a))$ and $\chi_{\infty}=\chi_{F_{\mathfrak{n}}}$. By the weak approximation theorem, we know that $F_{\mathfrak{n}}$ is dense in $\left(\mathbb{R}^{\times}\right)^{d}$. Thus $\chi_{\infty}$ is uniquely determined by $\chi$.

For the second assertion, we suppose that there exists a pair of characters $\left(\chi_{f}, \chi_{\infty}\right)$ satisfying $\chi_{f}(a) \chi_{\infty}(a)=1$ for all $a \in \mathcal{O}_{F}^{\times}$. We define $\chi_{0}: \mathcal{O}(\mathfrak{n}) \longrightarrow S^{1}$ by

$$
\chi_{0}(a)=\chi_{f}(a) \chi_{\infty}(a)
$$

Then we extend $\chi_{0}$ to $F(\mathfrak{n})$ by

$$
\chi_{0}\left(a b^{-1}\right)=\chi_{0}(a) \chi_{0}(b)^{-1}
$$

One can define $\chi_{0}$ on $P(\mathfrak{n})$ by setting $\chi_{0}\left(\left(a b^{-1}\right)\right)=\chi_{0}\left(a b^{-1}\right)$. It is obvious that $\chi_{0}$ is a character of $P(\mathfrak{n})$. Let $\operatorname{Ind}_{P(\mathfrak{n})}^{I_{\mathfrak{n}}} \chi_{0}:=\mathbb{C}\left[I_{\mathfrak{n}}\right] \otimes_{\mathbb{C}[P(\mathfrak{n})]} \chi_{0}$ be the induced representation. Since $I_{\mathfrak{n}}$ is an abelian group, $\operatorname{Ind}_{P(\mathfrak{n})}^{I_{\mathfrak{n}}} \chi_{0}$ decomposes into one-dimensional representations $\chi$ with finite part $\chi_{f}$ and infinite part $\chi_{\infty}$. Note that $\chi$ need not be unique. It is easy to see that the quotient of any such $\chi$ is a character of the finite group $I_{\mathfrak{n}} / P(\mathfrak{n})$.

Proposition 2.1.5. Let $\chi$ be a Grossencharacter $\bmod \mathfrak{n}$, and let $\mathfrak{m}$ be an integral ideal of $F$ dividing n. Then the following are equivalent.

1. $\chi$ is the restriction of a Grossencharacter $\chi^{\prime} \bmod \mathfrak{m}$. 
2. $\chi_{f}$ factors through $\left(\mathcal{O}_{F} / \mathfrak{m}\right)^{\times}$.

For a proof, see [Neu, Proposition 6.2].

A Grossencharacter $\chi \bmod \mathfrak{n}$ is called primitive if it is not the restriction of a Grossencharacter character $\chi^{\prime} \bmod \mathfrak{m}$ for any proper ideal $\mathfrak{m}$ dividing $\mathfrak{n}$. The conductor of $\chi$ is the smallest divisor $\mathfrak{f}$ of $\mathfrak{n}$ such that $\chi_{f}$ factors through $\left(\mathcal{O}_{F} / \mathfrak{f}\right)^{\times}$.

Next we describe characters on $\left(\mathbb{R}^{\times}\right)^{d}$.

Proposition 2.1.6. Every character $\chi$ of $\left(\mathbb{R}^{\times}\right)^{d}$ is given by

$$
\chi(x)=\prod_{j=1}^{d} \operatorname{sgn}\left(x_{j}\right)^{r_{j}}\left|x_{j}\right|^{i s_{j}}
$$

for some $r_{j} \in\{0,1\}$ and $s_{j} \in \mathbb{R}$ uniquely determined by $\chi$.

Proof. Since $\left(\mathbb{R}^{\times}\right)^{d} \simeq\{ \pm 1\}^{d} \times\left(\mathbb{R}^{+}\right)^{d}$, and characters of $\{ \pm 1\}^{d}$ are of the form $\prod_{j=1}^{g} \operatorname{sgn}^{r_{j}}$ for some $r_{j} \in\{0,1\}$, it is enough to describe characters of $\left(\mathbb{R}^{+}\right)^{d}$, where $\mathbb{R}^{+}$is the set of all positive real numbers. It is well-known that all characters of $\mathbb{R}$ are of the form $x \mapsto e^{i s x}$ for some $s \in \mathbb{R}$. The assertion follows from the isomorphism $\mathbb{R}^{+} \simeq \mathbb{R} ; x \mapsto \log |x|$.

Corollary 2.1.7. Let $\chi$ be a Grossencharacter with finite part $\chi_{f}$ and infinite part $\chi_{\infty}$, and let the notation be as above. Then the following are equivalent:

1. $\chi$ is of finite order.

2. $\chi_{\infty}$ is of finite order.

3. $\chi_{\infty}$ has finite image.

4. $s_{j}=0$ for all $j=1, \ldots, d$. 
5. $\chi_{\infty}$ is trivial on $\left(\mathbb{R}^{+}\right)^{d}$.

ProOF. It follows from Proposition 2.1.6 that (2)-(5) are equivalent. It remains to show (1) and (2) are equivalent. We suppose that $\chi$ is of finite order. Then every element $a \in F_{\mathfrak{n}}$ satisfies $\chi_{\infty}(a)=\chi((a))$. Since $F_{\mathfrak{n}}$ is dense in $\left(\mathbb{R}^{\times}\right)^{d}, \chi$ is of finite order implies $\chi_{\infty}$ is of finite order.

Conversely, we assume that $\chi_{\infty}$ is of finite order. Let $m$ be the cardinality of $I_{\mathfrak{n}} / P(\mathfrak{n})^{+}$, where $P(\mathfrak{n})^{+}=\left\{(a) \mid a \in F_{\mathfrak{n}}^{+}\right\}$, where $F_{\mathfrak{n}}^{+}$is the set of totally positive elements in $F_{\mathfrak{n}}$. It is known that $m$ is finite. Then for any $\mathfrak{a} \in I_{\mathfrak{n}}, \mathfrak{a}^{m}=(a)$ for some $a \in F_{\mathfrak{n}}^{+}$so $\chi_{\infty}(a)=1$ by $(5)$, and it is clear that $\chi_{f}(a)=1$. Hence, $\chi(\mathfrak{a})^{m}=1$ for all $\mathfrak{a} \in I_{\mathfrak{n}}$ so $\chi$ is of finite order.

\section{Narrow ray class characters}

Definition 2.1.8. A modulus $\mathfrak{n}$ of $F$ is a formal product of all places of $F$

$$
\mathfrak{n}=\prod_{\mathfrak{p}} \mathfrak{p}^{e_{\mathfrak{p}}}=\mathfrak{n}_{\infty} \cdot \mathfrak{n}_{f}
$$

where

1. $e_{\mathfrak{p}} \in \mathbb{Z}_{\geq 0}$ and $e_{\mathfrak{p}}=0$ for almost all $\mathfrak{p}$.

2. $e_{\mathfrak{p}} \in\{0,1\}$ for real places $\mathfrak{p}$ of $F$.

Here $\mathfrak{n}_{\infty}=\prod_{\mathfrak{p} \mid \infty} \mathfrak{p}^{e_{\mathfrak{p}}}$ is a subset of the real places of $F$, and $\mathfrak{n}_{f}=\prod_{\mathfrak{p}<\infty} \mathfrak{p}^{e_{\mathfrak{p}}}$ is an integral ideal of $F$.

Given a modulus $\mathfrak{n}$, we define $I_{\mathfrak{n}}=I_{\mathfrak{n}_{f}}$ and $P_{\mathfrak{n}}$ as the subgroup of all principal ideals $(a)$ with $a \in F^{\times}$such that $a \equiv 1 \bmod \mathfrak{n}_{f}$ and $\tau_{\mathfrak{p}}(a)>0$ for all real embeddings $\tau_{\mathfrak{p}}$ with $\mathfrak{p} \mid \mathfrak{n}_{\infty}$

The ray class group modulo $\mathfrak{n}$ is defined as $\mathrm{Cl}_{\mathfrak{n}}=I_{\mathfrak{n}} / P_{\mathfrak{n}}$. If $e_{\mathfrak{p}}=1$ for all $\mathfrak{p} \mid \infty$, we call $\mathrm{Cl}_{\mathfrak{n}}$ the narrow ray class group modulo $\mathfrak{n}$ and denote it by $\mathrm{Cl}_{\mathfrak{n}_{f}}^{+}$. We see that $\mathrm{Cl}_{F}=\mathrm{Cl}_{\mathcal{O}_{F}}$ and $\mathrm{Cl}_{F}^{+}=\mathrm{Cl}_{\mathcal{O}_{F}}^{+}$. By a narrow ray class character mod $\mathfrak{n}$ we mean a character of $\mathrm{Cl}_{\mathfrak{n}_{f}}^{+}$, the narrow ray class group modulo 
$\mathfrak{n}$. A narrow ray class character $\chi$ modulo $\mathfrak{n}$ is said to be primitive if it does not factor through $\mathrm{Cl}_{\mathfrak{m}_{f}}^{+}$ for any integral ideal $\mathfrak{m}_{f}$ dividing $\mathfrak{n}_{f}$. In such a case, we call $\mathfrak{n}_{f}$ the conductor of $\chi$.

Proposition 2.1.9. The narrow ray class characters mod $\mathfrak{n}$ are exactly the Grossencharacters $\chi$ mod $\mathfrak{n}$ of finite order. Moreover, the conductor of a narrow ray class character is the same as the conductor of the corresponding Grossencharacter.

PROOF. Let $\chi$ be a Grossencharacter $\bmod \mathfrak{n}$ of finite order. To see $\chi$ is a narrow ray class character, it suffices to show that $\chi$ is trivial on $P_{\mathfrak{n}}$. We have seen that $\chi_{f}(a)=1$ for $a \in F_{\mathfrak{n}}($ Lemma 2.1.1 and the definition of $\left.\chi_{f}\right)$, and $\chi_{\infty}(a)=1$ for $a \in F^{+}$(Corollary 2.1.7) and hence, $\chi((a))=1$ for all $a \in P_{\mathfrak{n}}$. Therefore, $\chi$ is a narrow ray class character $\bmod \mathfrak{n}$.

Conversely, let $\chi$ be a narrow ray class character $\bmod \mathfrak{n}$. To show $\chi$ is a Grossencharacter, we have to find its finite part $\chi_{f}$ and its infinite part $\chi_{\infty}$. Note that by weak approximation theorem, we have

$$
F_{\mathfrak{n}} / F_{\mathfrak{n}}^{+} \simeq\left(\mathbb{R}^{\times}\right)^{d} /\left(\mathbb{R}^{+}\right)^{d} \simeq\{ \pm 1\}^{d}
$$

Then the restriction of $\chi$ to $F_{\mathfrak{n}} / F_{\mathfrak{n}}^{+}$induces a character $\chi_{\infty}:\left(\mathbb{R}^{\times}\right)^{d} \longrightarrow S^{1}$. We define

$$
\chi_{f}(a)=\chi((a)) \chi_{\infty}^{-1}(a)
$$

for $a \in \mathcal{O}(\mathfrak{n})$. Since $\chi_{\infty}(a)=\chi((a))$ for all $a \in F_{\mathfrak{n}}, \chi_{f}$ is a character of $F(\mathfrak{n}) / F_{\mathfrak{n}} \simeq\left(\mathcal{O}_{F} / \mathfrak{n}\right)^{\times}$. Therefore, $\chi$ is a Grossencharacter. The last assertion follows directly from the correspondence described above.

\section{Hecke characters}

Definition 2.1.10. A Hecke character $\chi$ is a character of the idele class group

$$
\chi: \mathbb{A}_{F}^{\times} / F^{\times} \longrightarrow S^{1}
$$


The following lemma is easily proven.

Lemma 2.1.11. Let $\chi_{\mathfrak{p}}$ be a character of $F_{\mathfrak{p}}^{\times}$. Then $\operatorname{ker} \chi_{\mathfrak{p}}$ contains $1+\varpi_{\mathfrak{p}}^{m} \mathcal{O}_{\mathfrak{p}}$ for a sufficiently large $m \in \mathbb{Z}_{\geq 0}$

If $f$ is the smallest positive integer satisfying Lemma 2.1.11, we call $\mathfrak{p}^{f}$ the conductor of $\chi_{\mathfrak{p}}$. A character $\chi_{\mathfrak{p}}$ is unramified if its conductor is $\mathcal{O}_{\mathfrak{p}}$, i.e., $\chi_{\mathfrak{p}}\left(\mathcal{O}_{\mathfrak{p}}^{\times}\right)=1$.

Lemma 2.1.12. Let $\chi$ be a Hecke character. There exists a family of continuous characters $\chi_{\mathfrak{p}}$ : $F_{\mathfrak{p}}^{\times} \longrightarrow S^{1}$ for the places $\mathfrak{p}$ of $F$ such that

(1) for almost all finite places $\mathfrak{p}$ of $F, \chi_{\mathfrak{p}}$ is unramified,

(2) if $x \in F^{\times}$, then $\prod_{\mathfrak{p}} \chi_{\mathfrak{p}}(x)=1$, and

(3) if $x=\left(x_{\mathfrak{p}}\right) \in \mathbb{A}^{\times}$, then $\chi(x)=\prod_{\mathfrak{p}} \chi_{\mathfrak{p}}\left(x_{\mathfrak{p}}\right)$.

The above products run through all places of $F$.

Conversely, any family of characters $\left\{\chi_{\mathfrak{p}}\right\}$ that satisfies (1) and (2) determines a unique Hecke character $\chi$ satisfying (3).

Proof. Let $\chi$ be a Hecke character. We define $\chi_{\mathfrak{p}}=\left.\chi\right|_{F_{\mathfrak{p}}}$. Since the topology of $F_{\mathfrak{p}}^{\times}$is the subspace topology of $\mathbb{A}_{F}^{\times}$, it follows from the continuity of $\chi$ that $\chi_{\mathfrak{p}}$ is also continuous. By the same argument as in Lemma 2.1.11, we see that the kernel of $\chi$ contains an open subgroup of $\mathbb{A}_{F}^{\times}$. Then $\chi_{\mathfrak{p}}$ is unramified for almost all finite places $\mathfrak{p}$. The properties (2) and (3) follow from the definition of Hecke characters and the construction of $\chi_{\mathfrak{p}}$.

Now suppose we are given a family of continuous characters $\left\{\chi_{\mathfrak{p}}\right\}$ satisfying (1) and (2). We define $\chi(x)=\prod_{\mathfrak{p}} \chi_{\mathfrak{p}}\left(x_{\mathfrak{p}}\right)$ for all $x=\left(x_{\mathfrak{p}}\right) \in \mathbb{A}_{F}^{\times}$. Then $\chi$ is a character of $\mathbb{A}_{F}^{\times}$and trivial on $F^{\times}$by $(2)$. Since 
$\chi_{\mathfrak{p}}$ is continuous for all $\mathfrak{p} \mid \infty$ it follows from (1) that $\chi$ is continuous at 1 , and hence, it is continuous everywhere.

Let $\mathfrak{n}=\prod_{\mathfrak{p} \mid \mathfrak{n}} \mathfrak{p}^{e_{\mathfrak{p}}}$ be an integral ideal of $F$, and let $\mathbb{A}_{f, \mathfrak{n}}^{\times}=\prod_{\mathfrak{p}<\infty}\left(1+\varpi_{\mathfrak{p}}^{e_{\mathfrak{p}}} \mathcal{O}_{\mathfrak{p}}\right)$, where $e_{\mathfrak{p}}=0$ if $\mathfrak{p} \nmid \mathfrak{n}$. Then a Hecke character $\chi$ with conductor $\mathfrak{n}$ is a character of $C_{\mathfrak{n}}=\mathbb{A}^{\times} / F^{\times} \mathbb{A}_{f, \mathfrak{n}}^{\times}$. Recall that we have a short exact sequence [Neu, Proposition 6.13, p. 482]

$$
1 \rightarrow F(\mathfrak{n}) / \mathcal{O}_{\mathfrak{n}}^{\times} \stackrel{i}{\rightarrow} I_{\mathfrak{n}} \times\left(\mathcal{O}_{F} / \mathfrak{n}\right)^{\times} \times\left(\mathbb{R}^{\times}\right)^{d} / \mathcal{O}_{\mathfrak{n}}^{\times} \stackrel{(\theta, \phi, \psi)}{\rightarrow} C_{\mathfrak{n}} \rightarrow 1
$$

where the map $i$ is defined by $i(a)=\left((a)^{-1}, a \bmod \mathfrak{n}, a \bmod \mathcal{O}_{\mathfrak{n}}^{\times}\right)$, the map $\theta: I_{\mathfrak{n}} \longrightarrow C_{\mathfrak{n}}$ is defined by $\theta(\mathfrak{a})=\left(\varpi_{\mathfrak{p}}^{\operatorname{val}_{\mathfrak{p}}(\mathfrak{a})}\right)$, the map $\phi:\left(\mathcal{O}_{F} / \mathfrak{n}\right)^{\times} \longrightarrow C_{\mathfrak{n}}$ is defined by $\phi(a)=\left(a_{\mathfrak{p}}\right)$ with

$$
a_{\mathfrak{p}}= \begin{cases}a & \text { if } \mathfrak{p} \nmid \mathfrak{n} \\ 1 & \text { if } \mathfrak{p} \mid \mathfrak{n} \\ \tau_{\mathfrak{p}}(a) & \text { if } \mathfrak{p} \mid \infty\end{cases}
$$

and the map $\psi:\left(\mathbb{R}^{\times}\right)^{d} / \mathcal{O}_{\mathfrak{n}}^{\times} \longrightarrow C_{\mathfrak{n}}$ is defined by $\psi(b)=b^{-1}$. One can use this short exact sequence to show that the following proposition holds.

\section{Proposition 2.1.13.}

1. For every integral $\mathfrak{n}$, the map $\chi \mapsto \chi^{*}=\chi \circ \theta$ is bijective between the set of all Hecke characters with conductor $\mathfrak{n}$ and the set of all Grossencharacters with conductor $\mathfrak{n}$.

2. For each $\mathfrak{p} \nmid \mathfrak{n}$, we have $\chi_{\mathfrak{p}}(x)=\chi^{*}\left(\mathfrak{p}^{\operatorname{val}_{\mathfrak{p}}(x)}\right)$ for $\mathfrak{p}<\infty$ and $\chi_{\mathfrak{p}}=\left(\chi_{\infty}^{*}\right)^{-1}$ for $\mathfrak{p} \mid \infty$.

3. If $\mathfrak{p} \mid \mathfrak{n}$, then $\left.\chi_{\mathfrak{p}}\right|_{\mathcal{O}_{\mathfrak{p}}^{\times}}=\left(\chi_{f}^{*(\mathfrak{p})}\right)^{-1}$, where $\chi^{*(\mathfrak{p})}$ is the restriction of $\chi_{f}^{*}$ to $\left(\mathcal{O}_{F} / \mathfrak{p}^{\mathrm{val}_{\mathfrak{p}}(\mathfrak{n})}\right)^{\times}$.

For a proof see [Neu, Corollary 6.14] 
Proposition 2.1.14. A character $\chi_{0}$ of $(\mathcal{O} / \mathfrak{n})^{\times}$lifts to a Hecke character $\chi$ of finite order with conductor dividing $\mathfrak{n}$ and satisfying

$$
\chi_{0}(a)=\prod_{\mathfrak{p} \mid \mathfrak{n}} \chi_{\mathfrak{p}}(a), a \in F(\mathfrak{n})
$$

if and only if $\chi_{0}$ is trivial on $\left(\mathcal{O}_{F}^{\times}\right)^{+}$. Moreover, if $\chi_{0}$ can be lifted, then there are exactly $h_{F}^{+}$liftings.

ProOF. The first assertion follows from the above discussion. To see there are exactly $h_{F}^{+}$liftings, we observe that if $\chi_{1}$ and $\chi_{2}$ are two different liftings, then $\chi_{1} \chi_{2}^{-1}$ is a character of the narrow class group. Thus the assertion follows.

\subsection{Some useful decompositions of $\mathrm{GL}_{2}$}

In this subsection, we review decompositions of $\mathrm{GL}_{2}$ for later use. We denote by $B$ the subgroup of $\mathrm{GL}_{2}$ consisting of all upper-triangular matrices.

Proposition 2.2.1 (Iwasawa decomposition for $\mathrm{GL}_{2}(\mathbb{R})$ ). Let the notation be as above. Then we have

$$
\mathrm{GL}_{2}(\mathbb{R})=B(\mathbb{R}) \mathrm{SO}_{2}(\mathbb{R})
$$

Proof. Given any $g=\left(\begin{array}{ll}a & b \\ c & d\end{array}\right) \in \mathrm{GL}_{2}(\mathbb{R})$, we have

$$
\left(\begin{array}{ll}
a & b \\
c & d
\end{array}\right)=\left(\begin{array}{cc}
\frac{\operatorname{det} g}{\sqrt{c^{2}+d^{2}}} & \frac{a c+b d}{\sqrt{c^{2}+d^{2}}} \\
0 & \sqrt{c^{2}+d^{2}}
\end{array}\right)\left(\begin{array}{cc}
\frac{d}{\sqrt{c^{2}+d^{2}}} & \frac{-c}{\sqrt{c^{2}+d^{2}}} \\
\frac{c}{\sqrt{c^{2}+d^{2}}} & \frac{d}{\sqrt{c^{2}+d^{2}}}
\end{array}\right) .
$$

This completes the proof.

For the remainder of this chapter, we let $K$ be a finite field extension of $\mathbb{Q}_{p}$, and we let $\mathcal{O}$ be the ring of integers of $K$ with uniformizer $\varpi$ and maximal ideal $\mathfrak{p}$. We denote by $q$ the cardinality of the residue field $\mathcal{O} / \varpi=: k$. We denote by val the normalized valuation satisfying $\operatorname{val}(\varpi)=1$ and denote by $|\cdot|$ the absolute value defined by $|\varpi|=q^{-\operatorname{val}(\varpi)}=q^{-1}$. 
Lemma 2.2.2 (Iwasawa decomposition for $\mathrm{GL}_{2}(K)$ ). Let the notation be as above. Then we have

$$
\mathrm{GL}_{2}(K)=B(K) \mathrm{GL}_{2}(\mathcal{O})
$$

Moreover, for each $\left(\begin{array}{ll}a & b \\ c & d\end{array}\right) \in \mathrm{GL}_{2}(K)$, we have

$$
\left(\begin{array}{ll}
a & b \\
c & d
\end{array}\right)=\left\{\begin{array}{cc}
\left(\begin{array}{cc}
a-\frac{b c}{d} & b \\
0 & d
\end{array}\right)\left(\begin{array}{cc}
1 & 0 \\
\frac{c}{d} & 1
\end{array}\right) & \text { if }|c| \leq|d|(\text { i.e., } \operatorname{val}(c) \geq \operatorname{val}(d)) \\
\left(\begin{array}{cc}
-\frac{a d}{c}+b & a \\
0 & c
\end{array}\right)\left(\begin{array}{cc}
0 & 1 \\
1 & \frac{d}{c}
\end{array}\right) & \text { if }|c| \geq|d| \text { (i.e., } \operatorname{val}(c) \leq \operatorname{val}(d)) .
\end{array}\right.
$$

Proof. For any $\left(\begin{array}{ll}a & b \\ c & d\end{array}\right) \in \mathrm{GL}_{2}(K)$, if $\operatorname{val}(c) \leq \operatorname{val}(d)$, then

$$
\left(\begin{array}{ll}
a & b \\
c & d
\end{array}\right)\left(\begin{array}{cc}
1 & 0 \\
-c / d & 1
\end{array}\right)=\left(\begin{array}{cc}
a-b c d^{-1} & b \\
0 & d
\end{array}\right)
$$

If $\operatorname{val}(c) \geq \operatorname{val}(d)$, then

$$
\left(\begin{array}{ll}
a & b \\
c & d
\end{array}\right)\left(\begin{array}{ll}
0 & 1 \\
1 & 0
\end{array}\right)=\left(\begin{array}{ll}
b & a \\
d & c
\end{array}\right)=\left(\begin{array}{cc}
b-a d c^{-1} & a \\
0 & c
\end{array}\right)\left(\begin{array}{cc}
1 & 0 \\
d / c & 1
\end{array}\right)
$$

Thus the assertion follows.

For $N \in \mathbb{Z}_{\geq 0}$, we let

$$
K_{1}\left(\mathfrak{p}^{N}\right)=\left\{\left(\begin{array}{ll}
a & b \\
c & d
\end{array}\right) \in \mathrm{GL}_{2}(\mathcal{O}) \mid c \equiv 0 \bmod \mathfrak{p}^{N}, d-1 \equiv 0 \bmod \mathfrak{p}^{N}\right\}
$$

Lemma 2.2.3. Let the notation be as above. For any $N \in \mathbb{N}$, we have

$$
\mathrm{GL}_{2}(\mathcal{O})=\bigcup_{i=0}^{N} B(\mathcal{O}) \gamma_{i} K_{1}\left(\mathfrak{p}^{N}\right)
$$

where $\gamma_{i}=\left(\begin{array}{rr}1 & 0 \\ \varpi^{i} & 1\end{array}\right)$ for $0 \leq i<N$ and $\gamma_{N}=I_{2}$, the identity matrix.

ProOF. We follow the argument in [Sch]. Given any $g=\left(\begin{array}{ll}a & b \\ c & d\end{array}\right) \in \mathrm{GL}_{2}(\mathcal{O})$, if $c$ is a unit, then we have

$$
\left(\begin{array}{ll}
a & b \\
c & d
\end{array}\right)=\left(\begin{array}{cc}
\frac{a d-b c}{c} & a+\frac{(b c-a d)\left(1+\varpi^{N}\right)}{c} \\
0 & c
\end{array}\right)\left(\begin{array}{ll}
1 & 0 \\
1 & 1
\end{array}\right)\left(\begin{array}{cc}
1+\varpi^{N} & \left(1+\varpi^{N}\right) c^{-1} d-1 \\
-\varpi^{N} & 1-\varpi^{N} c^{-1} d
\end{array}\right) .
$$


If $\operatorname{val}(c)=j>0$, then we have

$$
\left(\begin{array}{ll}
a & b \\
c & d
\end{array}\right)=\left(\begin{array}{cc}
(a d-b c) c^{-1} \varpi^{j} & b \\
0 & d
\end{array}\right)\left(\begin{array}{cc}
1 & 0 \\
\varpi^{j} & 1
\end{array}\right)\left(\begin{array}{cc}
\varpi^{-j} c d^{-1} & 0 \\
0 & 1
\end{array}\right)
$$

Note that $g \in \mathrm{GL}_{2}(\mathcal{O})$ implies that $\operatorname{det} g$ is a unit in $\mathcal{O}$, i.e., $\operatorname{val}(\operatorname{det} g)=0$. On the other hand, $\operatorname{det} g=a d-b c$ and $\operatorname{val}(a d-b c) \geq \min \{\operatorname{val}(a d), v(b c)\}$ with equality when $\operatorname{val}(a d) \neq v(b c)$. It follows from $\operatorname{val}(c)=j$ that $\operatorname{val}(b c) \geq j$. Thus $\operatorname{val}(a d)=0$, i.e., $a$ and $d$ are units in $\mathcal{O}$. Therefore, the above decomposition makes sense.

\subsection{Local integrals}

In Chapter 4, we will construct Eisenstein series adelically and compute their constant terms at different cusps. This involves to computing local integrals. In this section, we compute some integrals as background for Chapter 4 .

We denote by $\operatorname{Tr}=\operatorname{Tr}_{K / \mathbb{Q}_{p}}$ the trace map from $K$ to $\mathbb{Q}_{p}$. For $a \in \mathbb{Q}_{p}$, we denote by $[a]_{p}$ the fractional part of $a$. In other words, if one writes $a=\sum_{i=-m}^{\infty} a_{i} p^{i}$, then $[a]_{p}=\sum_{i=-m}^{-1} a_{i} p^{i}$.

We fix the additive character $\psi: K^{\times} \longrightarrow \mathbb{C}^{\times}$defined as

$$
\psi(x)=e^{-2 \pi i[\operatorname{Tr}(x)]_{p}}
$$

We say that $\psi$ is of conductor $\varpi^{-r}$ if $r$ is the smallest positive integer such that $\psi\left(\varpi^{-r} \mathcal{O}_{v}\right)=1$ or equivalently, $r$ is the smallest positive integer such that $\operatorname{Tr}\left(\varpi^{-r}\right) \in \mathbb{Z}_{p}$. Indeed, $\mathfrak{p}^{-r}$ is the inverse different of $K$. For $u \in K^{\times}$, we define the additive character $\psi_{u}: K \longrightarrow \mathbb{C}^{\times}$by setting $\psi_{u}(x)=\psi(u x)$ for all $x \in K$. Note that the conductor of $\psi_{u}$ is $\varpi^{-r-\operatorname{val}(u)}$.

We denote by $d x$ be the normalized additive Haar measure such that the volume of $\mathcal{O}$ is 1.

Proposition 2.3.1. Let the notation be as above. Then we have 
(1) $\int_{\mathcal{O} \times} 1 d x=\frac{q-1}{q}$

(2) $\int_{\varpi^{k} \mathcal{O}^{\times}}|x|^{s} d x=\frac{q-1}{q} \times q^{-k(s+1)}$ for $k \in \mathbb{Z}$ and $s \in \mathbb{C}$.

(3) For $u \in K$, we define $\gamma(u)=\int_{\mathcal{O}} \psi_{u}(-x) d x$. Then we have

$$
\gamma(u)= \begin{cases}1 & \text { if } u \in \varpi^{-r} \mathcal{O} \\ 0 & \text { otherwise. }\end{cases}
$$

(4) Let the notation be as in 3. Then we have $\int_{\varpi^{k} \mathcal{O}} \psi_{u}(-x) d x=q^{-k} \gamma\left(u \varpi^{k}\right)$ for $u \in K$ and $k \in \mathbb{Z}$.

Proof.

1. By writing $\mathcal{O}^{\times}$as $\cup_{u \in k} \times u+\varpi \mathcal{O}$ and by substitution, we have

$$
\int_{\mathcal{O}^{\times}} 1 d x=\sum_{u \in k^{\times}} \int_{u+\varpi \mathcal{O}} 1 d x=\sum_{u \in k^{\times}} q^{-1} \int_{\mathcal{O}} 1 d x=\frac{q-1}{q} .
$$

2. By substitution, we have

$$
\int_{\varpi^{k} \mathcal{O} \times}|x|^{s} d x=|\varpi|^{k(s+1)} \int_{\mathcal{O}^{\times}} 1 d x=q^{-k(s+1)} \frac{q-1}{q} .
$$

3. If $u \in \varpi^{-r} \mathcal{O}$, then we have $\psi_{u}(x)=1$ for all $x \in \mathcal{O}$. Thus the assertion follows from the first assertion. Now, we suppose that $u \notin \varpi^{-r} \mathcal{O}$. That is $u \in \varpi^{-n} \mathcal{O}^{\times}$for some positive integer $n>r$. Then the conductor of $\psi_{u}$ is $\varpi^{n-r}$, and hence, we have

$$
\int_{\mathcal{O}} \psi_{u}(-x) d x=\sum_{y \in \mathcal{O} / \varpi^{n-r} \mathcal{O}} \psi_{u}(-y) \int_{\varpi^{n-r} \mathcal{O}} 1 d x
$$

Since $\psi_{u}$ is a nontrivial character of $\mathcal{O} / \varpi^{n-r} \mathcal{O}$, we have $\sum_{y \in \mathcal{O} / \varpi^{n-r} \mathcal{O}} \psi_{u}(-y)=0$, from which it follows that $\int_{\mathcal{O}} \psi_{u}(-x) d x=0$.

4. The assertion follows from the substitution that $x=\varpi^{k} y$ for $y \in \mathcal{O}$ and the third assertion. 


\subsection{Local epsilon factors}

Local epsilon factors show up naturally in the construction of Eisenstein series. In this subsection, we review the definition of local epsilon factors and their properties. The main reference for this section is Tate's thesis [Cas-Fro].

Let the notation be as in the previous section. Let $\theta$ be a character of $K^{\times}$. If $\theta$ is unramified then the local epsilon factor $\varepsilon(s, \theta, \psi)$ is defined to be 1 . If $\theta$ is ramified of conductor $\varpi^{e}$, then $\varepsilon(s, \theta, \psi)$ is defined as [Tate]

$$
\varepsilon(s, \theta, \psi)=\int_{K^{\times}}|x|^{-s} \theta^{-1}(x) \psi(x) d x .
$$

This integral converges absolutely when $\operatorname{re}(s)>3 / 2$ and has analytic continuation to all $s$ without having any zeros [B, Proposition 3.1.9].

For a positive integer $n$, we put $U^{(n)}:=\mathcal{O}^{\times} /\left(1+\varpi^{n} \mathcal{O}\right)$. Assume that $\theta$ is a ramified primitive character of conductor $\varpi^{e}$. We set

$$
\tau(\theta):=\sum_{x \in U^{(e)}} \theta(x) \psi\left(\varpi^{-e-r} x\right) .
$$

Note that the definition of $\tau(\theta)$ does not depends on the choice of representatives $x$. This follows from an observation that

$$
\psi\left(\varpi^{-e-r}\left(x+\varpi^{e}\right)\right)=\psi\left(\varpi^{-e-r} x+\varpi^{-r}\right)=\psi\left(\varpi^{-e-r} x\right) .
$$

The following lemma is well-known, and one can use it to write local epsilon factors as character sums. This will be addressed in next section.

Lemma 2.4.1. Let the notation be as above. Assume that the character $\theta$ is ramified of conductor 
$\varpi^{e}$. Then we have

$$
\int_{\varpi^{m} \mathcal{O}^{\times}} \theta(x) \psi(x) d x= \begin{cases}\left|\varpi^{-r}\right| \theta\left(\varpi^{-r-e}\right) \tau(\theta) & \text { if } m=-r-e \\ 0 & \text { if } m \neq-r-e .\end{cases}
$$

ProOF. We follow the computation in [H2, p. 259]. If $m=-r-e$, then we have

$$
\begin{aligned}
\int_{\varpi^{-r-e} \mathcal{O}^{\times}} \theta(x) \psi(x) d x & =\left|\varpi^{-r-e}\right| \int_{\mathcal{O}^{\times}} \theta\left(\varpi^{-r-e} x\right) \psi\left(\varpi^{-r-e} x\right) d x \\
& =\left|\varpi^{-r-e}\right| \theta\left(\varpi^{-r-e}\right) \sum_{x \in U^{(e)}} \theta(x) \int_{1+\varpi^{e} \mathcal{O}} \psi\left(\varpi^{-r-e} x h\right) d h \\
& =\left|\varpi^{-r-e}\right| \theta\left(\varpi^{-r-e}\right) \sum_{x \in U^{(e)}} \theta(x) \int_{\varpi^{e} \mathcal{O}} \psi\left(\varpi^{-r-e} x(H+1)\right) d H \\
& =\left|\varpi^{-r}\right| \theta\left(\omega^{-r-e}\right) \sum_{x \in U^{(e)}} \theta(x) \int_{\mathcal{O}} \psi\left(\omega^{-r-e} x\left(\varpi^{e} H+1\right)\right) d H \\
& =\left|\varpi^{-r}\right| \theta\left(\varpi^{-r-e}\right) \sum_{x \in U^{(e)}} \theta(x) \psi\left(\varpi^{-r-e} x\right) \int_{\mathcal{O}} \psi\left(\varpi^{-r} x H\right) d H \\
& ={ }^{(*)}\left|\varpi^{-r}\right| \theta\left(\varpi^{-r-e}\right) \sum_{x \in U^{(e)}} \theta(x) \psi\left(\varpi^{-r-e} x\right) \\
& =\left|\varpi^{-r}\right| \theta\left(\varpi^{-r-e}\right) \tau(\theta) .
\end{aligned}
$$

Here the equality $(*)$ is obtained by Proposition 2.3.1(3).

Now we assume $m \neq-r-e$. By substituting $x=\varpi^{m} y$ for $y \in \mathcal{O}^{\times}$, we have

$$
\int_{\varpi^{m} \mathcal{O}^{\times}} \theta(x) \psi(x) d x=\left|\varpi^{m}\right| \theta\left(\varpi^{m}\right) \int_{\mathcal{O}^{\times}} \theta(y) \psi\left(\varpi^{m} y\right) d y .
$$

To show the assertion, it suffices to show that $\int_{\mathcal{O}^{\times}} \theta(x) \psi\left(\varpi^{m} x\right) d x=0$. 
If $m<-r-e$, then we have

$$
\begin{aligned}
\int_{\mathcal{O}^{\times}} \theta(x) \psi\left(\varpi^{m} x\right) d x & =\sum_{x \in U^{(e)}} \theta(x) \int_{1+\varpi^{e} \mathcal{O}} \psi\left(\varpi^{m} x h\right) d h \\
& =\sum_{x \in U^{(e)}} \theta(x) \int_{\varpi^{e} \mathcal{O}} \psi\left(\varpi^{m} x(H+1)\right) d H \\
& =\sum_{x \in \mathcal{O}^{\times} / 1+\varpi^{e} \mathcal{O}} \theta(x) \psi\left(\varpi^{m} x\right) \int_{\varpi^{e} \mathcal{O}} \psi\left(\varpi^{m} x H\right) d H \\
& =\left|\varpi^{e}\right| \sum_{x \in U^{(e)}} \theta(x) \psi\left(\varpi^{m} x\right) \int_{\mathcal{O}} \psi\left(\varpi^{m+e} x y\right) d y .
\end{aligned}
$$

Since we have $m+e<-r$, Proposition 2.3.1(3) implies that

$$
\int_{\mathcal{O}} \psi\left(\varpi^{m+e} x y\right) d y=0
$$

Finally, we consider the case $m>-r-e$.

$$
\begin{aligned}
\int_{\mathcal{O}^{\times}} \theta(x) \psi\left(\varpi^{m} x\right) d x & =\sum_{x \in U^{(e-1)}} \int_{1+\varpi^{e-1} \mathcal{O}} \theta(x n) \psi\left(\varpi^{m} x n\right) d n \\
& =\sum_{x \in U^{(e-1)}} \int_{\varpi^{e-1} \mathcal{O}} \theta(x(y+1)) \psi\left(\varpi^{m} x(y+1)\right) d y \\
& =\sum_{x \in U^{(e-1)}} \theta(x) \psi\left(\varpi^{m} x\right) \int_{\varpi^{e-1} \mathcal{O}} \theta(y+1) \psi\left(\varpi^{m} x y\right) d y \\
& =\left|\varpi^{e-1}\right| \sum_{x \in U^{(e-1)}} \theta(x) \psi\left(\varpi^{m} x\right) \int_{\mathcal{O}} \theta\left(\varpi^{e-1} y+1\right) \psi\left(\varpi^{m+e-1} x y\right) d y .
\end{aligned}
$$

Since we have $m+e-1 \geq-r$, we know that $\psi\left(\varpi^{m+e-1} x y\right)=1$ for all $x \in U^{(e-1)}$ and $y \in \mathcal{O}$.

Hence, we have

$$
\int_{\mathcal{O}} \theta\left(\varpi^{e-1} y+1\right) \psi\left(\varpi^{m+e-1} x y\right) d y=\int_{\mathcal{O}} \theta\left(\varpi^{e-1} y+1\right) d y .
$$

To complete the proof, we show that $\int_{\mathcal{O}} \theta\left(1+\varpi^{e-1} y\right) d y=0$. It is easy to see that

$$
\int_{1+\varpi^{e-1} \mathcal{O}} \theta(y) d y=\left|\varpi^{e-1}\right| \int_{\mathcal{O}} \theta\left(1+\varpi^{e-1} y\right) d y
$$


Moreover, we have

$$
\int_{1+\varpi^{e-1} \mathcal{O}} \theta(y) d y=\sum_{x \in 1+\varpi^{e-1} \mathcal{O} / 1+\varpi^{e} \mathcal{O}} \theta(x) \int_{1+\varpi^{e} \mathcal{O}} 1 d y
$$

Since $\theta$ is a nontrivial character of conductor $\varpi^{e}$, we have

$$
\sum_{x \in 1+\varpi^{e-1} \mathcal{O} / 1+\varpi^{e} \mathcal{O}} \theta(x)=0,
$$

and hence, $\int_{\mathcal{O}} \theta\left(1+\varpi^{e-1} y\right) d y=0$. This completes the proof.

\subsection{Character sums}

The computation of congruence modules associated to Eisenstein series involves proving certain properties of character sums. In order to make our computations easy to follow, we prove those properties in this section.

Let the notation be as before. Let $\theta_{1}$ and $\theta_{2}$ be two ramified primitive characters of $K^{\times}$of conductor $\mathfrak{p}^{e_{1}}$ and $\mathfrak{p}^{e_{2}}$, respectively. We set

$$
J_{a}\left(\theta_{1}, \theta_{2}, \varpi^{k}\right):=\sum_{x \in U^{(k)}} \theta_{1}(x) \theta_{2}(a-x)
$$

for $a \in \mathcal{O}$ and $k \geq \max \left\{e_{1}, e_{2}\right\} \in \mathbb{Z}_{>0}$. It is easy to see that $J_{a}\left(\theta_{1}, \theta_{2}, \varpi^{k}\right)=J_{a}\left(\theta_{2}, \theta_{1}, \varpi^{k}\right)$, and $J_{a}\left(\theta_{1} \theta, \theta_{2}, \varpi^{k}\right)=J_{a}\left(\theta_{1}, \theta_{2}, \varpi^{k}\right)$ if $\theta$ is unramified.

In order to simplify the proof of Lemma 2.5.2, we will sometimes view the character $\theta_{2}$ as a character of $\left(\mathcal{O} / \varpi^{e_{2}} \mathcal{O}\right)^{\times}$by the isomorphism $U^{\left(e_{2}\right)} \simeq\left(\mathcal{O} / \varpi^{e_{2}} \mathcal{O}\right)^{\times}$. One can extend it to a character of $\mathcal{O} / \varpi^{e_{2}} \mathcal{O}$ by setting $\theta_{2}(x)=0$ if $x \equiv 0 \bmod \varpi$. Then we have $\tau\left(\theta_{2}\right)=\sum_{x \in \mathcal{O} / \varpi^{e_{2} \mathcal{O}}} \theta_{2}(x) \psi\left(\varpi^{-e_{2}-r} x\right)$.

Lemma 2.5.1. Let the notation be as above. Then we have

$$
\sum_{y \in \mathcal{O} / \varpi^{e_{2} \mathcal{O}}} \theta_{2}(y) \psi\left(\varpi^{-r-e_{2}} x y\right)= \begin{cases}\theta_{2}^{-1}(x) \tau\left(\theta_{2}\right) & \text { if } x \in \mathcal{O}^{\times} \\ 0 & \text { if } x \in \varpi \mathcal{O} .\end{cases}
$$


Proof. The assertion is well-known for Dirichlet characters. We repeat the proof for the reader's convenience. If $x \in \mathcal{O}^{\times}$, one has

$$
\sum_{y \in \mathcal{O} / \varpi^{e_{2} \mathcal{O}}} \theta_{2}(y) \psi\left(\varpi^{-r-e_{2}} x y\right)=\sum_{t \in \mathcal{O} / \varpi^{e_{2} \mathcal{O}}} \theta_{2}\left(t x^{-1}\right) \psi\left(\varpi^{-r-e_{2}} t\right)=\theta_{2}^{-1}(x) \tau\left(\theta_{2}\right)
$$

Now we assume that $\operatorname{val}(x)=g>0$ and write $x=\varpi^{g} x^{\prime}$ for some $x^{\prime} \in \mathcal{O}^{\times}$. If $e_{2}<g$, then $\psi\left(\varpi^{-r-e_{2}+g} x^{\prime} y\right)=1$ for all $x^{\prime} \in \mathcal{O}^{\times}$and $y \in \mathcal{O} / \varpi^{e_{2}} \mathcal{O}$. It is easy to see that

$$
\sum_{y \in \mathcal{O} / \varpi^{e_{2} \mathcal{O}}} \theta_{2}(y) \psi\left(\varpi^{-r-e_{2}} x y\right)=\sum_{y \in \mathcal{O} / \varpi^{e_{2} \mathcal{O}}} \theta_{2}(y) \psi\left(\varpi^{-r-e_{2}+g} x^{\prime} y\right)=0
$$

If $e_{2} \geq g$, then we have

$$
\begin{aligned}
\sum_{y \in \mathcal{O} / \varpi^{e_{2} \mathcal{O}}} \theta_{2}(y) \psi\left(\varpi^{-r-e_{2}} x y\right) & =\sum_{y \in \mathcal{O} / \varpi^{e_{2} \mathcal{O}}} \theta_{2}(y) \psi\left(\varpi^{-r-e_{2}+g} x^{\prime} y\right)\left(\text { set } s=x^{\prime} y\right) \\
& =\theta_{2}^{-1}\left(x^{\prime}\right) \sum_{s \in \mathcal{O} / \varpi^{e_{2} \mathcal{O}}} \theta_{2}(s) \psi\left(\varpi^{-r-e_{2}+g} s\right) \\
& =\theta_{2}^{-1}\left(x^{\prime}\right) \sum_{s \in \mathcal{O} / \varpi^{e_{2}-g} \mathcal{O}}\left(\sum_{\substack{s^{\prime} \in \mathcal{O} / \varpi^{e_{2} \mathcal{O}} \\
s^{\prime} \equiv s \bmod \varpi^{e_{2}-g}}} \theta_{2}\left(s^{\prime}\right)\right) \psi\left(\varpi^{-r-e_{2}+g} s\right) .
\end{aligned}
$$

We claim that

$$
\sum_{\substack{s^{\prime} \in \mathcal{O} / \varpi^{e_{2} \mathcal{O}} \\ s^{\prime} \equiv s \bmod \varpi^{e_{2}-g}}} \theta_{2}\left(s^{\prime}\right)=0
$$

for all $s \in \mathcal{O} / \varpi^{e_{2}-g} \mathcal{O}$. We set

$$
S=\left\{s \in \mathcal{O} / \varpi^{e_{2}} \mathcal{O} \mid s \equiv 1 \bmod \varpi^{e_{2}-g}\right\}
$$

Since $\theta_{2}$ is primitive, $\sum_{s \in S} \theta_{2}(s)=0$. Therefore, we have

$$
\sum_{\substack{s^{\prime} \in \mathcal{O} / \varpi^{e_{2}} \\ s^{\prime} \equiv s \operatorname{Ood} \varpi^{e_{2}-g}}} \theta_{2}\left(s^{\prime}\right)=\sum_{s^{\prime} \in s S} \theta_{2}\left(s^{\prime}\right)=0
$$

for all $s \in \mathcal{O} / \varpi^{e_{2}-g} \mathcal{O}$. This completes the proof. 
Lemma 2.5.2. Let the notation be as above.

1. If $\theta_{2}$ is ramified, then $\varepsilon\left(s, \theta_{2}^{-1}, \psi\right)=|\varpi|^{s\left(r+e_{2}\right)-r} \theta_{2}\left(\varpi^{-r-e_{2}}\right) \tau\left(\theta_{2}\right)$.

2. We have $\tau\left(\theta_{2}\right) \overline{\tau\left(\theta_{2}\right)}=N\left(\mathfrak{p}^{e_{2}}\right)$.

3. Assume that both $\theta_{1}$ and $\theta_{2}$ are ramified, and assume that $e_{1}=e_{2}$. Let $\varpi^{s}$ be the conductor of $\theta_{1}^{-1} \theta_{2}$. Furthermore, if $1 \leq s \leq e_{1}$, then

$$
J_{\varpi^{e_{1}-s}}\left(\theta_{1}^{-1}, \theta_{2}, \varpi^{e_{1}}\right)=\tau\left(\theta_{1}^{-1}\right) \tau\left(\theta_{2}\right) / \tau\left(\theta_{1}^{-1} \theta_{2}\right)
$$

4. Assume that both $\theta_{1}$ and $\theta_{2}$ are ramified. If $e_{1} \neq e_{2}$, then we have

$$
\tau\left(\theta_{1}^{-1}\right) \tau\left(\theta_{2}\right)=\tau\left(\theta_{1}^{-1} \theta_{2}\right) \times \begin{cases}\sum_{x \in U^{\left(e_{2}\right)}} \theta_{2}(x) \theta_{1}^{-1}\left(1-\varpi^{e_{1}-e_{2}} x\right) & \text { if } e_{1}>e_{2} \\ \sum_{x \in U^{\left(e_{1}\right)}} \theta_{1}^{-1}(x) \theta_{2}\left(1-\varpi^{e_{2}-e_{1}} x\right) & \text { if } e_{1}<e_{2}\end{cases}
$$

ProOF.

1. Note that

$$
\int_{\varpi^{-r-e_{2} \mathcal{O} \times}} \theta_{2}(x)|x|^{-s} \psi(x) d x=|\varpi|^{s\left(r+e_{2}\right)} \int_{\varpi^{-r-e_{2} \mathcal{O} \times}} \theta_{2}(x) \psi(x) d x .
$$

Then the assertion follows from Lemma 2.4.1.

2. We have

$$
\tau\left(\theta_{2}\right) \overline{\tau\left(\theta_{2}\right)}=\sum_{x \in \mathcal{O} / \varpi^{e_{2} \mathcal{O}}} \theta_{2}^{-1}(x) \tau\left(\theta_{2}\right) \psi\left(-\varpi^{-r-e_{2}} x\right) .
$$

By Lemma 2.5.1, we have

$$
\theta_{2}^{-1}(x) \tau\left(\theta_{2}\right)=\sum_{y \in \mathcal{O} / \varpi^{e_{2} \mathcal{O}}} \theta_{2}(y) \psi\left(\varpi^{-r-e_{2}} x y\right)
$$


for all $x \in U^{\left(e_{2}\right)}$. Thus, we have

$$
\begin{aligned}
\tau\left(\theta_{2}\right) \overline{\tau\left(\theta_{2}\right)} & =\sum_{x \in \mathcal{O} / \varpi^{e_{2}} \mathcal{O}} \sum_{y \in \mathcal{O} / \varpi^{e_{2} \mathcal{O}}} \theta_{2}(y) \psi\left(\varpi^{-r-e_{2}} x y\right) \psi\left(-\varpi^{-r-e_{2}} x\right) \\
& =\sum_{y \in \mathcal{O} / \varpi^{e_{2}} \mathcal{O}} \theta_{2}(y) \sum_{x \in \mathcal{O} / \varpi^{e_{2}} \mathcal{O}} \psi\left(\varpi^{-r-e_{2}}(y-1) x\right) \\
& =N\left(\mathfrak{p}^{e_{2}}\right) .
\end{aligned}
$$

The last equality holds since when $y=1$ the second sum is $N\left(\mathfrak{p}^{e_{2}}\right)$ and when $y \neq 1$ then second sum is 0 .

3. For a proof, see [Jun, Theorem 2.5].

4. Since the first and the second cases are equivalent, we only deal with the second case. Assume that $e_{1}>e_{2}$. Then we have

$$
\begin{aligned}
\tau\left(\theta_{1}^{-1}\right) \tau\left(\theta_{2}\right) & =\left(\sum_{x \in U^{\left(e_{1}\right)}} \theta_{1}^{-1}(x) \psi\left(\varpi^{-r-e_{1}} x\right)\right)\left(\sum_{y \in U^{\left(e_{2}\right)}} \theta_{2}(y) \psi\left(\varpi^{-r-e_{2}} y\right)\right) \\
& =\left(\sum_{x \in U^{\left(e_{1}\right)}} \theta_{1}^{-1}(x) \psi\left(\varpi^{-r-e_{1}} x\right)\right)\left(N\left(\varpi^{e_{1}-e_{2}}\right)^{-1} \sum_{y \in U^{\left(e_{1}\right)}} \theta_{2}(y) \psi\left(\varpi^{-r-e_{2}} y\right)\right) \\
& =N\left(\varpi^{e_{1}-e_{2}}\right)^{-1} \sum_{x, y \in U^{\left(e_{1}\right)}} \theta_{1}^{-1}(x) \theta_{2}(y) \psi\left(\varpi^{-r-e_{1}}\left(x+\varpi^{e_{1}-e_{2}} y\right)\right) \quad\left(\text { let } z=x+\varpi^{e_{1}-e_{2}} y\right) \\
& =N\left(\varpi^{e_{1}-e_{2}}\right)^{-1} \sum_{y, z \in U^{\left(e_{1}\right)}} \theta_{1}^{-1}\left(z-\varpi^{e_{1}-e_{2}} y\right) \theta_{2}(y) \psi\left(\varpi^{-r-e_{1}} z\right) \quad(\text { let } y=w z) \\
& =N\left(\varpi^{e_{1}-e_{2}}\right)^{-1} \sum_{w, z \in U^{\left(e_{1}\right)}} \theta_{1}^{-1}\left(z-\varpi^{e_{1}-e_{2}} w z\right) \theta_{2}(w z) \psi\left(\varpi^{-r-e_{1}} z\right) \\
& =N\left(\varpi^{e_{1}-e_{2}}\right)^{-1} \sum_{w \in U^{\left(e_{1}\right)}} \theta_{1}^{-1}\left(1-\varpi^{e_{1}-e_{2}} w\right) \theta_{2}(w) \sum_{z \in U^{\left(e_{1}\right)}} \theta_{1}^{-1} \theta_{2}(z) \psi\left(\varpi^{-r-e_{1}} z\right) \\
& =\sum_{w \in U^{\left(e_{2}\right)}} \theta_{1}^{-1}\left(1-\varpi^{e_{1}-e_{2}} w\right) \theta_{2}(w) \times \tau\left(\theta_{1}^{-1} \theta_{2}\right) .
\end{aligned}
$$


The next lemma will be used in the proof of Lemma 4.1.5.

Lemma 2.5.3. Let the notation be as above.

1. If $e_{1}>e_{2}$, then we have

$$
\int_{\mathcal{O}^{\times}} \theta_{1}\left(1+\varpi^{e_{1}-e_{2}} x\right) \theta_{2}(x) d x=|\varpi|^{e_{2}} \sum_{x \in U^{\left(e_{2}\right)}} \theta_{2}(x) \theta_{1}\left(1+\varpi^{e_{1}-e_{2}} x\right) .
$$

2. If $e_{1}=e_{2}$, then we have

$$
\int_{\mathcal{O}^{\times}} \theta_{1}\left(\varpi^{k-e_{1}}+x\right) \theta_{2}(x) d x=|\varpi|^{e_{1}} \sum_{x \in U^{\left(e_{1}\right)}} \theta_{2}(x) \theta_{1}\left(\varpi^{k-e_{1}}+x\right)
$$

for all positive integers $k \geq e_{1}$.

ProOF. Since the computations for both assertions are essentially the same, we will only prove the first assertion. Assume that $e_{1}>e_{2}>0$. Then we have

$$
\begin{aligned}
\int_{\mathcal{O}^{\times}} \theta_{1}\left(1+\varpi^{e_{1}-e_{2}} x\right) \theta_{2}(x) d x & =\sum_{x \in U^{\left(e_{2}\right)}} \theta_{2}(x) \int_{1+\varpi^{e_{2} \mathcal{O}}} \theta_{1}\left(1+\varpi^{e_{1}-e_{2}} x y\right) d y \\
& =\sum_{x \in U^{\left(e_{2}\right)}} \theta_{2}(x) \theta_{1}\left(1+\varpi^{e_{1}-e_{2}} x\right) \int_{\mathcal{O}} 1 d\left(\varpi^{e_{2}} z\right) \\
& =\left|\varpi^{e_{2}}\right| \sum_{x \in U^{\left(e_{2}\right)}} \theta_{2}(x) \theta_{1}\left(1+\varpi^{e_{1}-e_{2}} x\right)
\end{aligned}
$$

Note that the second equality is obtained by letting $y=1+\varpi^{e_{2}} z$ and by the observation that

$$
\theta_{1}\left(1+\varpi^{e_{1}-e_{2}} x y\right)=\theta_{1}\left(1+\varpi^{e_{1}-e_{2}} x+\varpi^{e_{1}} z\right)=\theta_{1}\left(1+\varpi^{e_{1}-e_{2}} x\right)
$$

for all $x \in \mathcal{O}^{\times}$and $z \in \mathcal{O}$. 


\section{CHAPTER 3}

Hilbert modular forms

In this chapter, we first review the definitions of classical Hilbert modular forms, adelic Hilbert modular forms and the Hecke action. We refer the reader to [Shi] for more details. Then we discuss the adelic formulation of cusps and the Hecke action on the set of cusps in the last subsection.

\subsection{Classical Hilbert modular forms}

Throughout this paper, we denote by $\mathbf{H}=\{z \in \mathbb{C} \mid \operatorname{im}(z)>0\}$ the complex upper half plane. Let

$$
\mathrm{GL}_{2}(F)^{+}=\left\{\gamma \in \mathrm{GL}_{2}(F) \mid \operatorname{det} \gamma \gg 0\right\}
$$

be the group of $2 \times 2$ matrices with totally positive determinant. Recall that $\tau_{1}, \ldots, \tau_{d}: F \hookrightarrow \mathbb{R}$ are fixed distinct real embeddings of $F$. The Hilbert modular group $\Gamma(1)$ is defined as

$$
\Gamma(1)=\mathrm{GL}_{2}\left(\mathcal{O}_{F}\right)^{+}:=\mathrm{GL}_{2}\left(\mathcal{O}_{F}\right) \cap \mathrm{GL}_{2}(F)^{+}
$$

For any integral ideal $\mathfrak{n}$ of $F$, let

$$
\Gamma(\mathfrak{n})=\left\{\gamma \in \mathrm{GL}_{2}\left(\mathcal{O}_{F}\right)^{+} \mid \gamma \equiv I_{2} \bmod \mathfrak{n}\right\}
$$

where $I_{2}$ is the $2 \times 2$ identity matrix. A subgroup $\Gamma \subset \mathrm{GL}_{2}(F)^{+}$is called a congruence subgroup if it contains $\Gamma(\mathfrak{n})$ for some nonzero integral ideal $\mathfrak{n}$ and is commensurable to $\Gamma(1)$. Recall that two subgroups $\Gamma_{1}$ and $\Gamma_{2}$ of some ambient group are commensurable if $\Gamma_{1} \cap \Gamma_{2}$ is of finite index in $\Gamma_{1}$ and in $\Gamma_{2}$. 
Let $\mathfrak{b}$ be a fractional ideal, and let $\mathfrak{n}$ be an integral ideal of $F$. We are interested in the congruence subgroups $\Gamma=\Gamma_{0}(\mathfrak{b}, \mathfrak{n}), \Gamma_{1}(\mathfrak{b}, \mathfrak{n})$, and $\Gamma_{1}^{1}(\mathfrak{b}, \mathfrak{n})$ which are defined by

$$
\begin{gathered}
\Gamma_{0}(\mathfrak{b}, \mathfrak{n})=\left\{\left(\begin{array}{ll}
a & b \\
c & d
\end{array}\right) \in \mathrm{GL}_{2}(F)^{+} \mid a, d \in \mathcal{O}_{F}, b \in \mathfrak{b}^{-1}, c \in \mathfrak{b} \mathfrak{n}, a d-b c \in \mathcal{O}_{F}^{\times}\right\}, \\
\Gamma_{1}(\mathfrak{b}, \mathfrak{n})=\left\{\left(\begin{array}{cc}
a & b \\
c & d
\end{array}\right) \in \Gamma_{0}(\mathfrak{b}, \mathfrak{n}) \mid d-1 \in \mathfrak{n}\right\},
\end{gathered}
$$

and

$$
\Gamma_{1}^{1}(\mathfrak{b}, \mathfrak{n})=\left\{\left(\begin{array}{ll}
a & b \\
c & d
\end{array}\right) \in \Gamma_{1}(\mathfrak{b}, \mathfrak{n}) \mid a-1 \in \mathfrak{n}\right\}
$$

The following lemma was proved by Dimitrov and Tilouine [DT, Lemma 1.4].

Lemma 3.1.1. Let the notation be as above. If $\mathfrak{n}$ is prime to $N(\mathfrak{b} \mathfrak{d})$ and if $\mathfrak{n}$ does not divide 2 and 3 , then the congruence subgroup $\Gamma_{1}(\mathfrak{b} \mathfrak{D}, \mathfrak{n})$ does not contain any nontrivial element of finite order.

Next we review the definition of classical Hilbert modular forms of parallel weight. Let $\chi_{0}$ : $\left(\mathcal{O}_{F} / \mathfrak{n}\right)^{\times} \longrightarrow \mathbb{C}^{\times}$be a character of finite order modulo $\mathfrak{n}$. This character induces a character on $\Gamma=\Gamma_{0}(\mathfrak{b}, \mathfrak{n})$ by setting

$$
\chi_{0}(\gamma)=\chi_{0}(d)
$$

where $\gamma=\left(\begin{array}{ll}a & b \\ c & d\end{array}\right) \in \Gamma$.

Let $f: \mathbf{H}^{d} \longrightarrow \mathbb{C}$ be a function. For $k \in \mathbb{Z}_{\geq 0}$ and $\gamma \in \mathrm{GL}_{2}(F)^{+}$, we define the slash operator as

$$
f \|_{k} \gamma(z):=(\operatorname{det} \gamma)^{k / 2} j(\gamma, z)^{-k} f(\gamma z)
$$

where

$$
(\operatorname{det} \gamma)^{k / 2}=\prod_{i=1}^{d} \tau_{i}(\operatorname{det} \gamma)^{k / 2}
$$

and similarly,

$$
j(\gamma, z)=\prod_{i=1}^{d}\left(\tau_{i}(c) z_{i}+\tau_{i}(d)\right) .
$$

Here $\gamma z=\left(\tau_{1}(\gamma) z_{1}, \ldots, \tau_{d}(\gamma) z_{d}\right)$, and $\tau_{i}(\gamma) z_{i}$ is the Mobius action on the upper half plane for all $i$. 
Definition 3.1.2. Let $\chi_{0}$ be a character of $\left(\mathcal{O}_{F} / \mathfrak{n}\right)^{\times}$. A Hilbert modular form of level $\Gamma_{0}(\mathfrak{b}, \mathfrak{n})$, (parallel) weight $k$ and character $\chi_{0}$ is a holomorphic function $f: \mathbf{H}^{d} \rightarrow \mathbb{C}$ such that

$$
f \|_{k} \alpha(z)=\chi_{0}(\alpha) f(z)
$$

for all $\alpha \in \Gamma_{0}(\mathfrak{b}, \mathfrak{n})$ and that is holomorphic at the cusps $\Gamma_{1}(\mathfrak{b}, \mathfrak{n}) \backslash \mathbb{P}^{1}(F)$.

It follows from the above definition that every Hilbert modular form $f$ satisfies $f(z)=f(z+a)$ for $a \in \mathfrak{b}^{-1}$. Hence one obtains the Fourier expansion

$$
f=\sum_{\mu \in \mathfrak{b} \mathfrak{D}^{-1}} c(\mu, f) e^{2 \pi i \operatorname{tr}(\mu z)},
$$

where $\operatorname{tr}(\mu z)=\sum_{i=1}^{d} \tau_{i}(\mu) z_{i}$. Note that when $F \neq \mathbb{Q}$, the holomorphicity at cusps automatically holds by the Koecher Principle which asserts that if $F \neq \mathbb{Q}$, then for all modular forms $f$, the Fourier coefficient $c(\mu, f)$ is non-zero if and only if

$$
\mu=0 \text { or } \mu \gg 0
$$

Therefore, we have

$$
f=\sum_{\mu \in\left(\mathfrak{b} \mathfrak{D}^{-1}\right)+\cup\{0\}} c(\mu, f) e^{2 \pi i \operatorname{tr}(\mu z)} .
$$

Definition 3.1.3. We say that a Hilbert modular form $f$ is a cusp form if the constant term in its Fourier expansion at each cusp vanishes, i.e., the constant term of $f \|_{k} \gamma$ vanishes for all $\gamma \in \mathrm{GL}_{2}(F)$.

For $R=\mathbb{C}$ or $\mathbb{Z}$, we denote by $M_{k}\left(\Gamma_{0}(\mathfrak{b}, \mathfrak{n}), \chi_{0} ; R\right)\left(\operatorname{resp} . S_{k}\left(\Gamma_{0}(\mathfrak{b}, \mathfrak{n}), \chi_{0} ; R\right)\right)$ the space of Hilbert modular forms (resp. cusp forms) of level $\Gamma_{0}(\mathfrak{b}, \mathfrak{n})$, weight $k$ and character $\chi_{0}$ whose Fourier coefficients are all in $R$. One can also define Hilbert modular forms of level $\Gamma_{1}(\mathfrak{b}, \mathfrak{n})$ or level $\Gamma_{1}^{1}(\mathfrak{b}, \mathfrak{n})$ in the same way. We denote by $M_{k}\left(\Gamma_{1}(\mathfrak{b}, \mathfrak{n}) ; R\right), M_{k}\left(\Gamma_{1}^{1}(\mathfrak{b}, \mathfrak{n}) ; R\right), S_{k}\left(\Gamma_{1}(\mathfrak{b}, \mathfrak{n}) ; R\right)$, and $S_{k}\left(\Gamma_{1}^{1}(\mathfrak{b}, \mathfrak{n}) ; R\right)$ the corresponding spaces. 
For any commutative ring $A$, we define

$$
M_{k}\left(\Gamma_{0}(\mathfrak{b}, \mathfrak{n}), \chi_{0} ; A\right):=M_{k}\left(\Gamma_{0}(\mathfrak{b}, \mathfrak{n}) ; \chi_{0}, \mathbb{Z}\right) \otimes_{\mathbb{Z}} A
$$

and define $S_{k}\left(\Gamma_{0}(\mathfrak{b}, \mathfrak{n}), \chi_{0} ; A\right), M_{k}\left(\Gamma_{1}(\mathfrak{b}, \mathfrak{n}) ; A\right), S_{k}\left(\Gamma_{1}(\mathfrak{b}, \mathfrak{n}) ; A\right), M_{k}\left(\Gamma_{1}^{1}(\mathfrak{b}, \mathfrak{n}) ; A\right)$, and $S_{k}\left(\Gamma_{1}^{1}(\mathfrak{b}, \mathfrak{n}) ; A\right)$ in the same manner.

\subsection{Adelic Hilbert modular forms}

Let $\mathbb{A}_{F}$ be the adele ring of $F$, and let $\mathbb{A}_{F, f}$ be the finite adele ring. For any finite place $v$ of $F$ and any integral ideal $\mathfrak{n}$ of $\mathcal{O}_{F}$, we define

$$
\begin{aligned}
& K_{0, v}(\mathfrak{n})=\left\{\left(\begin{array}{ll}
a & b \\
c & d
\end{array}\right) \in \mathrm{GL}_{2}\left(\mathcal{O}_{F_{v}}\right) \mid c \in \mathfrak{n}_{v},\right\}, \\
& K_{1, v}(\mathfrak{n})=\left\{\left(\begin{array}{ll}
a & b \\
c & d
\end{array}\right) \in K_{0, v}(n) \mid d-1 \in \mathfrak{n}_{v}\right\},
\end{aligned}
$$

and

$$
K_{v}^{1}(\mathfrak{n})=\left\{\left(\begin{array}{ll}
a & b \\
c & d
\end{array}\right) \in K_{0, v}(n) \mid a-1 \in \mathfrak{n}_{v}\right\}
$$

Let $K_{0}(\mathfrak{n})=\prod_{v<\infty} K_{0, v}(\mathfrak{n})$, and let $K_{1}(\mathfrak{n})=\prod_{v<\infty} K_{1, v}(\mathfrak{n})$. The group $K_{v}^{1}$ will only be used in the proof of Corollary 3.4.3. Each character $\chi_{0}$ of $(\mathcal{O} / \mathfrak{n})^{\times}$induces a character, also denoted by $\chi_{0}$, of $K_{0}(\mathfrak{n})$ by setting

$$
\chi_{0}\left(\left(\begin{array}{ll}
a & b \\
c & d
\end{array}\right)\right)=\chi_{0}\left(d_{\mathfrak{n}} \bmod \mathfrak{n}\right)
$$

where $d_{\mathfrak{n}}$ denotes the $\mathfrak{n}$-part of $d$.

Let $\mathrm{GL}_{2}^{+}(\mathbb{R})$ be the subgroup of $\mathrm{GL}_{2}(\mathbb{R})$ consisting all matrices with totally positive determinant, and let $K_{\infty}^{+}=\left(\mathbb{R}_{+}^{\times} \mathrm{SO}_{2}(\mathbb{R})\right)^{d}$. Note that $K_{\infty}^{+}$is the stabilizer of $(i, \ldots, i) \in \mathbf{H}^{d}$ in $\left(\mathrm{GL}_{2}^{+}(\mathbb{R})\right)^{d}$. The Hilbert modular variety for $K=K_{0}(\mathfrak{n})$ or $K_{1}(\mathfrak{n})$ is defined as

$$
Y_{K}=\mathrm{GL}_{2}(F) \backslash \mathrm{GL}_{2}\left(\mathbb{A}_{F}\right) / K_{\infty}^{+} K
$$


Definition 3.2.1. Let $k \in \mathbb{Z}_{\geq 0}$, and let $\chi_{0}$ be a character of $(\mathcal{O} / \mathfrak{n})^{\times}$. An adelic Hilbert modular form of weight $k$, level $K_{0}(\mathfrak{n})$, and character $\chi_{0}$ is a function $f: \mathrm{GL}_{2}\left(\mathbb{A}_{F}\right) \longrightarrow \mathbb{C}$ such that the following properties hold:

1. $f(\gamma g \kappa)=\chi_{0}(\kappa) f(g)$ for all $\gamma \in \mathrm{GL}_{2}(F), g \in \mathrm{GL}_{2}\left(\mathbb{A}_{F}\right)$, and $\kappa \in K_{0}(\mathfrak{n})$.

2. $f(g a)=(\operatorname{det} a)^{\frac{k}{2}} j(a, i)^{-k} f(g)$ for all $a \in\left(\mathrm{GL}_{2}^{+}(\mathbb{R})\right)^{d}$ and $g \in \mathrm{GL}_{2}\left(\mathbb{A}_{F}\right)$.

3. For $x \in \mathrm{GL}_{2}\left(\mathbb{A}_{F, f}\right)$, we define a function $f_{x}: \mathbf{H}^{d} \longrightarrow \mathbb{C}$ by

$$
f_{x}(z)=(\operatorname{det} g)^{-\frac{k}{2}} j(g, i)^{k} f(x g)
$$

for $g=\left(g_{j}\right) \in\left(\mathrm{GL}_{2}^{+}(\mathbb{R})\right)^{d}$ and $z=\left(z_{j}\right) \in \mathbf{H}^{d}$ such that $g_{j} i=z_{j}$ for $j=1, \ldots, d$. Then $f_{x}$ is a holomorphic function for all $x$.

An adelic Hilbert modular form $f$ is called a cusp form if we have

$$
\int_{F \backslash \mathbb{A}_{F}} f\left(\left(\begin{array}{cc}
1 & x \\
0 & 1
\end{array}\right) g\right) d x=0
$$

for almost all $g \in \mathrm{GL}_{2}\left(\mathbb{A}_{F}\right)$.

We denote by $M_{k}\left(K_{0}(\mathfrak{n}), \chi_{0} ; \mathbb{C}\right)$ the space of adelic Hilbert modular forms of weight $k$, level $K_{0}(\mathfrak{n})$, and character $\chi_{0}$ and denote by $S_{k}\left(K_{0}(\mathfrak{n}), \chi_{0} ; \mathbb{C}\right)$ the subspace of cusp forms. The following lemma is well-known [Shi, §1].

Lemma 3.2.2. Let the notation be as above. Then the space $M_{k}\left(K_{0}(\mathfrak{n}), \chi_{0} ; \mathbb{C}\right)$ is trivial unless $\chi_{0}$ satisfies the condition

$$
\chi_{0}(u)=\operatorname{sgn}(u)^{k}
$$


for all $u \in \mathcal{O}_{F}^{\times}$, where $\operatorname{sgn}(u)=\prod_{i=1}^{d} \operatorname{sgn}\left(\tau_{i}(u)\right)$ for $u \in F^{\times}$and

$$
\operatorname{sgn}(r)= \begin{cases}1 & \text { if } r>0 \\ -1 & \text { if } r<0\end{cases}
$$

for $r \in \mathbb{R}^{\times}$.

Proof. Suppose there is an element $f \neq 0$ in $M_{k}\left(K_{0}(\mathfrak{n}), \chi_{0} ; \mathbb{C}\right)$. For each $u \in \mathcal{O}_{F}^{\times}$, we have

$$
f\left(\left(\begin{array}{ll}
u & 0 \\
0 & u
\end{array}\right) g\right)=f\left(g\left(\begin{array}{ll}
u & 0 \\
0 & u
\end{array}\right)\right)
$$

for all $g \in \mathrm{GL}_{2}\left(\mathbb{A}_{F}\right)$. Here, on the left-hand side, we view $\left(\begin{array}{ll}u & 0 \\ 0 & u\end{array}\right) \in \mathrm{GL}_{2}(F)$; while on the right-hand side, we view $\left(\begin{array}{ll}u & 0 \\ 0 & u\end{array}\right) \in \mathrm{GL}_{2}\left(\mathbb{A}_{F}\right)$ via the diagonal embedding. It follows from Definition 3.2.1 that the left-hand side is $f(g)$, and the right-hand side is

$$
\chi_{0}(u)\left(\prod_{j=1}^{d} \tau_{j}\left(u^{2}\right)^{k / 2} \tau_{j}(u)^{-k}\right) f(g)=\chi_{0}(u) \operatorname{sgn}(u)^{k} f(g),
$$

for all $g \in \mathrm{GL}_{2}\left(\mathbb{A}_{F}\right)$. Thus $\chi_{0}$ has to satisfy $\chi_{0}(u)=\operatorname{sgn}(u)^{k}$ for all $u \in \mathcal{O}_{F}^{\times}$.

By strong approximation, the determinant map induces a bijection [Shi, §2]

$$
\mathrm{GL}_{2}(F) \backslash \mathrm{GL}_{2}\left(\mathbb{A}_{F}\right) / K\left(\mathrm{GL}_{2}^{+}(\mathbb{R})\right)^{d} \simeq F^{\times} \backslash \mathbb{A}_{F}^{\times} /\left(\operatorname{det} K \cdot \mathbb{R}_{+}^{d}\right) \simeq \mathrm{Cl}_{F}^{+}
$$

for $K=K_{1}(\mathfrak{n})$ or $K_{0}(\mathfrak{n})$. Let $\delta \in \mathbb{A}_{F, f}^{\times}$be such that $\delta_{v}=\varpi^{\text {val }_{v}(\mathfrak{D})}$ for $v<\infty$. Throughout this paper, we fix a set $\left\{t_{\lambda}\right\}_{\lambda=1, \ldots, h_{F}^{+}}$of representatives of the narrow class group of $F$ for some $t_{\lambda} \in \mathbb{A}_{F, f}^{\times}$such that $t_{\lambda}$ is prime to $\mathfrak{n}$ for $\lambda=1, \ldots, h_{F}^{+}$. Here we also denote by $t_{\lambda}$ the fractional ideal $t_{\lambda} \mathcal{O}_{F}$. We set

$$
x_{\lambda}=\left(\begin{array}{cc}
t_{\lambda} \delta & 0 \\
0 & 1
\end{array}\right) \in \mathrm{GL}_{2}\left(\mathbb{A}_{F, f}\right) .
$$

Then by (3.3), we see that $\left\{x_{\lambda}\right\}_{\lambda=1}^{h_{F}^{+}}$is a set of representatives of the double cosets $\mathrm{GL}_{2}(F) \backslash \mathrm{GL}_{2}\left(\mathbb{A}_{F}\right) / K\left(\mathrm{GL}_{2}^{+}(\mathbb{R})\right)^{d}$. We obtain

$$
\mathrm{GL}_{2}\left(\mathbb{A}_{F}\right)=\coprod_{\lambda=1}^{h_{F}^{+}} \mathrm{GL}_{2}(F) x_{\lambda}^{-1} K\left(\mathrm{GL}_{2}^{+}(\mathbb{R})\right)^{d}
$$


Again by (3.3), the equality (3.4) still holds if one removes $\delta$ from $x_{\lambda}$ for $\lambda=1, \ldots, h_{F}^{+}$. The reason we involve $\delta$ here is because it makes the computation in Chapter 4 simpler.

The following proposition is well-known [Shi, §2].

Proposition 3.2.3. Let the notation be as above. Then we have

$$
\Gamma_{0}\left(t_{\lambda} \mathfrak{D}, \mathfrak{n}\right)=x_{\lambda}^{-1}\left(\mathrm{GL}_{2}^{+}(\mathbb{R})\right)^{d} K_{0}(\mathfrak{n}) x_{\lambda} \cap \mathrm{GL}_{2}(F)
$$

and the map

$$
\begin{gathered}
\coprod_{\lambda=1}^{h_{F}^{+}} \Gamma_{0}\left(t_{\lambda} \mathfrak{D}, \mathfrak{n}\right) \backslash\left(\mathrm{GL}_{2}^{+}(\mathbb{R})\right)^{d} / K_{\infty}^{+} \longrightarrow \mathrm{GL}_{2}(F) \backslash \mathrm{GL}_{2}\left(\mathbb{A}_{F}\right) / K_{0}(\mathfrak{n}) K_{\infty}^{+} ; \\
\Gamma_{0}\left(t_{\lambda} \mathfrak{D}, \mathfrak{n}\right) g_{\infty} K_{\infty}^{+} \mapsto \mathrm{GL}_{2}(F) g_{\infty} x_{\lambda}^{-1} K_{0}(\mathfrak{n}) K_{\infty}^{+}
\end{gathered}
$$

is bijective. In particular, we have $Y_{K_{0}(\mathfrak{n})}=\coprod_{\lambda=1}^{h_{F}^{+}} \Gamma_{0}\left(t_{\lambda} \mathfrak{D}, \mathfrak{n}\right) \backslash \mathbf{H}^{d}$. Moreover, the same assertion also holds for $\Gamma_{1}\left(t_{\lambda} \mathfrak{D}, \mathfrak{n}\right)$ and $K_{1}(\mathfrak{n})$.

Proof. The first assertion follows from the definition of $\Gamma_{0}\left(t_{\lambda} \mathfrak{D}, \mathfrak{n}\right)$. For the second assertion, it follows from (3.4) that every element of $\mathrm{GL}_{2}\left(\mathbb{A}_{F}\right)$ is of the form $\mathrm{GL}_{2}(F) x_{\lambda}^{-1} g_{\infty} K_{0}(\mathfrak{n})$ for some $\lambda=1, \ldots, h_{F}^{+}$and for some $g_{\infty} \in\left(\mathrm{GL}_{2}^{+}(\mathbb{R})\right)^{d}$. Thus the map (3.5) is surjective. It remains to check that the map is injective. For simplicity, we write $\Gamma_{0}\left(t_{\lambda} \mathfrak{D}, \mathfrak{n}\right)$ as $\Gamma_{\lambda}$ and write $K_{0}(\mathfrak{n})$ as $K$. Suppose that $\Gamma_{\lambda} g_{\infty}$ and $\Gamma_{\lambda} g_{\infty}^{\prime}$ map to the same coset. Then there exist $\gamma \in \mathrm{GL}_{2}(F)$ and $k \in K$ such that $g_{\infty}^{\prime} x_{\lambda}^{-1}=\gamma g_{\infty} x_{\lambda}^{-1} k$. Thus, the finite part and infinite part of $\gamma$ satisfy

$$
\gamma_{f}=x_{\lambda}^{-1} k^{-1} x_{\lambda} \text { and } \gamma_{\infty}=g_{\infty}^{\prime} g_{\infty}^{-1}
$$

The former implies that $\gamma$ satisfies the congruence properties of $\Gamma_{\lambda}$, and the latter implies that $\gamma$ is totally positive. Hence, $\gamma \in \Gamma_{\lambda}$. 
The following proposition states the relationship between classical Hilbert modular forms and adelic Hilbert modular forms. See [Shi, §2] for a proof.

Proposition 3.2.4. Let $\chi_{0}$ be a character of $(\mathcal{O} / \mathfrak{n O})^{\times}$. If $\mathfrak{n}$ is prime to $\mathfrak{D}$, then there exist isomorphisms of complex vector spaces

$$
M_{k}\left(K_{0}(\mathfrak{n}), \chi_{0} ; \mathbb{C}\right) \simeq \bigoplus_{\lambda=1}^{h_{F}^{+}} M_{k}\left(\Gamma_{0}\left(t_{\lambda} \mathfrak{D}, \mathfrak{n}\right), \chi_{0, \lambda} ; \mathbb{C}\right)
$$

and

$$
S_{k}\left(K_{0}(\mathfrak{n}), \chi_{0} ; \mathbb{C}\right) \simeq \bigoplus_{i=1}^{h_{F}^{+}} S_{k}\left(\Gamma_{0}\left(t_{\lambda} \mathfrak{D}, \mathfrak{n}\right), \chi_{0, \lambda} ; \mathbb{C}\right),
$$

where $\chi_{0, \lambda}(\gamma)=\chi_{0}\left(x_{\lambda} \gamma x_{\lambda}^{-1}\right)$ for all $\gamma \in K_{0}(\mathfrak{n})$.

ProOF. Let $\left(f_{1}, \ldots, f_{h_{F}^{+}}\right) \in \prod_{\lambda} M_{k}\left(\Gamma_{0}\left(t_{\lambda} \mathfrak{D}, \mathfrak{n}\right), \chi_{0, \lambda} ; \mathbb{C}\right)$. We define a function $f \in M_{k}\left(K_{0}(\mathfrak{n}), \chi_{0} ; \mathbb{C}\right)$ by

$$
f\left(\alpha x_{\lambda}^{-1} u \kappa\right)=\left.f_{\lambda}\right|_{k} u(i) \chi_{0, \lambda}(\kappa) \text { for } \alpha \in \mathrm{GL}_{2}(F), u \in\left(\mathrm{GL}_{2}^{+}(\mathbb{R})\right)^{d}, \kappa \in K_{0}(\mathfrak{n})
$$

It follows from (3.4) that $f$ is well-defined.

Conversely, for any $f \in M_{k}\left(K_{0}(\mathfrak{n}), \chi_{0} ; \mathbb{C}\right)$, we associate $f_{\lambda}$ for $x_{\lambda}$ by Definition 3.2.1.

Remark 3.2.5. One can define the spaces of classical modular forms of level $\Gamma_{1}(\mathfrak{b} \mathfrak{D}, \mathfrak{n})$ and adelic modular forms of level $K_{1}(\mathfrak{n})$ in the same manner. The above discussion is also true by a similar argument.

From now on, we assume that $\mathfrak{n}$ is prime to $\mathfrak{D}$. Recall that $t_{1}, \ldots, t_{h_{F}^{+}}$are prime to $\mathfrak{n}$. Hence $\mathfrak{n}$ is prime to $t_{\lambda} \mathfrak{D}$ for all $\lambda=1, \ldots, h_{F}^{+}$. By Proposition 3.2.4, each $f \in M_{k}\left(K_{0}(\mathfrak{n}), \chi_{0} ; \mathbb{C}\right)$ can be written as a vector $\left(f_{1}, \ldots, f_{h_{F}^{+}}\right)$. We know that each $f_{\lambda}$ admits a Fourier expansion, namely,

$$
f_{\lambda}(z)=\sum_{\mu \in t_{\lambda}^{+} \cup\{0\}} c\left(\mu, f_{\lambda}\right) e^{2 \pi \operatorname{tr}(u z)}
$$


We call $c\left(\mu, f_{\lambda}\right)$ the unnormalized Fourier coefficients of $f$, and we define the normalized Fourier coefficients as follows. Each integral ideal $\mathfrak{m}$ of $F$ must be in one of the narrow ideal classes, say $\left(t_{\lambda} \mathfrak{D}\right)^{-1}$ for some $\lambda \in 1, \ldots, h_{F}^{+}$. We choose a totally positive element $u \in t_{\lambda} \mathfrak{D}$ such that $\mathfrak{m}=$ $(u)\left(t_{\lambda} \mathfrak{D}\right)^{-1}$. Then the normalized Fourier coefficient of $f$ associated to $\mathfrak{m}$ is defined by

$$
C(\mathfrak{m}, f):=N\left(t_{\lambda} \mathfrak{D}\right)^{-k / 2} c\left(u, f_{\lambda}\right)
$$

and the normalized constant terms are defined by

$$
C_{\lambda}(0, f):=N\left(t_{\lambda} \mathfrak{D}\right)^{-k / 2} c\left(0, f_{\lambda}\right)
$$

for $\lambda=1, \ldots, h_{F}^{+}$. The following lemma is well-known [Shi, $\left.\S 1\right]$.

Lemma 3.2.6. Let the notation be as above. Then $C(\mathfrak{m}, f)$ and $C_{\lambda}(0, f)$ for $\lambda=1, \ldots, h_{F}^{+}$are independent of the choice of $u$ and of the choices of the $t_{\lambda}$ representing ideal classes of the narrow class group.

Let $R=\mathbb{Z}\left[\chi_{0}\right]$ be an extension of $\mathbb{Z}$ containing of all values of $\chi_{0}$. We denote by $M_{k}\left(K_{0}(\mathfrak{n}), \chi_{0} ; R\right)$ the subspace of $M_{k}\left(K_{0}(\mathfrak{n}), \chi_{0} ; \mathbb{C}\right)$ consisting all modular forms whose Fourier coefficients are in $R$, and let $S_{k}\left(K_{0}(\mathfrak{n}), \chi_{0} ; R\right)$ be defined as in the same manner. For any $R$-algebra $A$, we define

$$
M_{k}\left(K_{0}(\mathfrak{n}), \chi_{0} ; A\right):=M_{k}\left(K_{0}(\mathfrak{n}), \chi ; R\right) \otimes_{R} A
$$

and

$$
S_{k}\left(K_{0}(\mathfrak{n}), \chi_{0} ; A\right):=S_{k}\left(K_{0}(\mathfrak{n}), \chi ; R\right) \otimes_{R} A
$$

We also define $M_{k}\left(K_{1}(\mathfrak{n}) ; A\right)$ and $S_{k}\left(K_{1}(\mathfrak{n}) ; A\right)$ in the same manner. 


\subsection{Hecke operators}

Let $K=K_{0}(\mathfrak{n})$ or $K_{1}(\mathfrak{n})$. In this subsection, we follow [Shi] to define the Hecke operators $S(\mathfrak{p}),\langle\mathfrak{p}\rangle$ and $T(\mathfrak{p})$ on $M_{k}(K ; \mathbb{C})$ for all prime ideals $\mathfrak{p}$ of $F$.

For any prime ideal $\mathfrak{p}$ of $F$, one has $K\left(\begin{array}{c}\varpi_{\mathfrak{p}} \\ 0 \\ 0\end{array}\right) K=\coprod_{i} \gamma_{i} K$ for some $\gamma_{i} \in \mathrm{GL}_{2}\left(F_{\mathfrak{p}}\right) \times \prod_{v \neq \mathfrak{p}}\left\{I_{v}\right\}$, where $I_{v}$ is the identity matrix in $\mathrm{GL}_{2}\left(F_{v}\right)$. For $f \in M_{k}(K, \mathbb{C})$, we define

$$
T^{\prime}(\mathfrak{p}) \cdot f(x)=\sum_{i} f\left(x \gamma_{i}\right)
$$

For instance, when $K=K_{0}(\mathfrak{n})$, it is well-known that we can take $\gamma_{i}$ as follows.

Lemma 3.3.1. Let the notation be as above. Then we have

$$
K_{0}(\mathfrak{n})\left(\begin{array}{cc}
\varpi_{\mathfrak{p}} & 0 \\
0 & 1
\end{array}\right) K_{0}(\mathfrak{n})= \begin{cases}\coprod_{u \in \mathcal{O}_{\mathfrak{p}} / \varpi_{\mathfrak{p}}}\left(\begin{array}{cc}
\varpi_{\mathfrak{p}} & u \\
0 & 1
\end{array}\right) K_{0}(\mathfrak{n}) \cup\left(\begin{array}{cc}
1 & 0 \\
0 & \varpi_{\mathfrak{p}}
\end{array}\right) K_{0}(\mathfrak{n}) & \text { if } \mathfrak{p} \nmid \mathfrak{n} \\
\coprod_{u \in \mathcal{O}_{\mathfrak{p}} / \varpi_{\mathfrak{p}}}\left(\begin{array}{cc}
\varpi_{\mathfrak{p}} & u \\
0 & 1
\end{array}\right) K_{0}(\mathfrak{n}) & \text { if } \mathfrak{p} \mid \mathfrak{n} .\end{cases}
$$

One can also define $T^{\prime}(\mathfrak{m})$ for all integral ideals $\mathfrak{m}$ in a similar manner. In this case, one will have a product of double cosets at places dividing $\mathfrak{m}$. See [Shi] for more information. If $\mathfrak{p} \nmid \mathfrak{n}$, we define

$$
S(\mathfrak{p}) \cdot f(x)=f\left(x \varpi_{\mathfrak{p}}\right)=f\left(x\left(\begin{array}{cc}
\varpi_{\mathfrak{p}} & 0 \\
0 & \varpi_{\mathfrak{p}}
\end{array}\right)\right)
$$

and define

$$
\langle\mathfrak{p}\rangle \cdot f(x)=f\left(x\left(\begin{array}{cc}
\varpi_{\mathfrak{p}} & 0 \\
0 & \varpi_{\mathfrak{p}}^{-1}
\end{array}\right)\right) .
$$

If $\mathfrak{p} \mid \mathfrak{n}$, we set $\langle\mathfrak{p}\rangle \cdot f=S(\mathfrak{p}) \cdot f=0$. Note that the definitions of $T^{\prime}(\mathfrak{p}),\langle\mathfrak{p}\rangle$ and $S(\mathfrak{p})$ do not depend on the choice of the uniformizer $\varpi_{\mathfrak{p}}$ at each place.

By Definition 3.2.1, we know that

$$
f(\text { aug })=\chi_{0}\left(u_{\mathfrak{n}} \bmod \mathfrak{n}\right) f(g)
$$


for $a \in F^{\times}$and $u \in \widehat{\mathcal{O}}_{F}^{\times}=\prod_{\mathfrak{p}<\infty} \mathcal{O}_{F_{\mathfrak{p}}}^{\times}$. Here $u_{\mathfrak{n}}=\prod_{\mathfrak{p} \mid \mathfrak{n}} u_{\mathfrak{p}}$ is the $\mathfrak{n}$-part of $u$. By this and Lemma 3.2.2, if $f \in M_{k}\left(K_{0}(\mathfrak{n}), \chi_{0} ; \mathbb{C}\right)$ is a nonzero common eigenform of $S(\mathfrak{p})$ for all prime ideals $\mathfrak{p}$ not dividing $\mathfrak{n}$, there is a unique narrow ray class character $\chi \bmod \mathfrak{n}$ with finite part $\chi_{0}^{-1}$ and infinite part $\chi_{\infty}=\operatorname{sgn}^{k}$ such that

$$
f(\alpha g)=\chi(\alpha) f(g)
$$

for all $\alpha \in \mathbb{A}_{F}^{\times}$. For each narrow ray class character $\chi \bmod \mathfrak{n}$, we denote by $M_{k}(\mathfrak{n}, \chi ; \mathbb{C})$ the space consisting of forms $f$ such that $f(\alpha g)=\chi(\alpha) f(g)$ for all $\alpha \in \mathbb{A}_{F}^{\times}$. Note that the relationship between $\chi$ and $\chi_{0}$ is given by

$$
\chi((a))=\chi_{0}(a)^{-1} \operatorname{sgn}^{k}(a)
$$

for all $a \in F^{\times}$such that the fractional ideal $(a)$ is prime to $\mathfrak{n}$. Moreover, the narrow ray class character $\chi$ is associated with a unique Hecke character $\tilde{\chi}: \mathbb{A}_{F}^{\times} \longrightarrow \mathbb{C}^{\times}$satisfying the following properties:

- $\tilde{\chi}\left(\varpi_{\mathfrak{p}}\right)=\chi(\mathfrak{p})$ for all $\mathfrak{p} \nmid \mathfrak{n}$.

- $\tilde{\chi}$ is unramified outside $\mathfrak{n}$.

- $\tilde{\chi}(u)=\chi_{f}^{-1}(u)$ for all $u \in \prod_{v \mid \mathfrak{n}} \mathcal{O}_{v}^{\times}$, where $\chi_{f}$ is the finite part of $\chi$.

- $\prod_{v} \widetilde{\chi}(a)=1$ for all $a \in F^{\times}$. Here $v$ runs through all places of $F$.

Throughout this paper, we will denote by $\chi$ the narrow ray class character and the associated Hecke character for simplicity.

For any integral ideal $\mathfrak{a}$, we put

$$
T(\mathfrak{a})=N(\mathfrak{a})^{(k-2) / 2} T^{\prime}(\mathfrak{a}) .
$$

It is known $[H 1, \S 3]$ that for any prime ideal $\mathfrak{p}$ not dividing $\mathfrak{n}, T(\mathfrak{p})$ and $S(\mathfrak{p})$ satisfy

$$
T(\mathfrak{p})^{2}-T\left(\mathfrak{p}^{2}\right)=N(\mathfrak{p}) S(\mathfrak{p})
$$


and it is known [Shi, (2.23)] that

$$
C(\mathfrak{m}, T(\mathfrak{a}) \cdot f)=\sum_{\mathfrak{m}+\mathfrak{a} \subset \mathfrak{b}} \chi(\mathfrak{b}) N(\mathfrak{b})^{k-1} C\left(\mathfrak{b}^{-2} \mathfrak{m} \mathfrak{a}, f\right)
$$

for all integral ideals $\mathfrak{a}$ and $\mathfrak{m}$.

\section{Definition 3.3.2.}

1. We say that a modular form $f$ is an eigenform if $f$ is an eigenvector for the Hecke operator $T(\mathfrak{m})$ for all integral ideals $\mathfrak{m}$ of $F$.

2. An eigenform $f$ is normalized if $C(1, f)=1$.

3. Let $\mathfrak{p}$ be a prime ideal of $F$. A normalized eigenform $f$ is called $\mathfrak{p}$-ordinary if its $\mathfrak{p t h}$ Fourier coefficient $C(\mathfrak{p}, f)$ is an unit in $\mathcal{O}_{\mathfrak{p}}$. A normalized eigenform $f$ is called $p$-ordinary if it is $\mathfrak{p}$-ordinary for all $\mathfrak{p} \mid p$.

We will write $T(\mathfrak{p})$ as $U(\mathfrak{p})$ if $\mathfrak{p}$ divides the level. Let

$$
e=\lim _{n \longrightarrow \infty} \prod_{\mathfrak{p} \mid p} U(\mathfrak{p})^{n !}
$$

be Hida's idempotent element. It was shown by Wiles [Wil2, p. 537] that for all $r \in \mathbb{Z}_{>0}$, $e$ acts on $M_{k}\left(K_{1}\left(\mathfrak{n} p^{r}\right) ; \mathbb{Z}_{p}\right)$ under the $p$-adic topology, and $S_{k}\left(K_{1}\left(\mathfrak{n} p^{r}\right) ; \mathbb{Z}_{p}\right)$ is invariant under the action of $e$. We denote by $M_{k}^{\text {ord }}\left(K_{1}\left(\mathfrak{n} p^{r}\right) ; \mathbb{Z}_{p}\right)$ the subspace $e \cdot M_{k}\left(K_{1}\left(\mathfrak{n} p^{r}\right) ; \mathbb{Z}_{p}\right)$ and similarly for $S_{k}^{\text {ord }}\left(K_{1}\left(\mathfrak{n} p^{r}\right) ; \mathbb{Z}_{p}\right)$. We denote by $\mathcal{H}_{k}\left(K_{1}\left(\mathfrak{n} p^{r}\right) ; \mathbb{Z}_{p}\right) \subset \operatorname{End}_{\mathbb{Z}_{p}}\left(M_{k}\left(K_{1}\left(\mathfrak{n} p^{r}\right) ; \mathbb{Z}_{p}\right)\right) \quad($ resp. $h_{k}\left(K_{1}\left(\mathfrak{n} p^{r}\right) ; \mathbb{Z}_{p}\right) \subset \operatorname{End}_{\mathbb{Z}_{p}}\left(S_{k}\left(K_{1}\left(\mathfrak{n} p^{r}\right) ; \mathbb{Z}_{p}\right)\right)$ the Hecke algebra (resp. cuspidal Hecke algebra) generated over $\mathbb{Z}_{p}$ by the Hecke operators $T(\mathfrak{q})$ and $S(\mathfrak{q})$ for all prime ideals $\mathfrak{q}$ of $F$. We write $H_{k}^{\text {ord }}\left(K_{1}\left(\mathfrak{n} p^{r}\right) ; \mathbb{Z}_{p}\right)=e \cdot H_{k}\left(K_{1}\left(\mathfrak{n} p^{r}\right) ; \mathbb{Z}_{p}\right)$ and write $h_{k}^{\text {ord }}\left(K_{1}\left(\mathfrak{n} p^{r}\right) ; \mathbb{Z}_{p}\right)$ in the same manner. 
We now review properties of Eisenstein series attached to pairs of narrow ray class characters of $F$. We refer the reader to [Shi, Proposition 3.4] for more details. Let $\chi_{1}$ and $\chi_{2}$ be primitive narrow ray class characters of conductors $\mathfrak{n}_{1}$ and $\mathfrak{n}_{2}$, respectively, with associated signs $q, r \in(\mathbb{Z} / 2 \mathbb{Z})^{d}$ satisfying

$$
q+r \equiv(k, \ldots, k)\left(\bmod 2 \mathbb{Z}^{d}\right)
$$

for some integer $k \geq 2$. We view the characters $\chi_{1} \chi_{2}$ and $\chi_{1} \chi_{2}^{-1}$ as characters modulo $\mathfrak{n}=\mathfrak{n}_{1} \mathfrak{n}_{2}$.

Proposition 3.3.3 (Shimura). Let the notation be as above. Assume $\chi_{1}$ is primitive. If $F=\mathbb{Q}$, we assume further that $\left(\chi_{1}, \chi_{2}, k\right) \neq(\mathbf{1}, \mathbf{1}, 2)$. Then for all $k \geq 2$, there exists an eigenform $E_{k}\left(\chi_{1}, \chi_{2}\right) \in$ $M_{k}\left(\mathfrak{n}, \chi_{1} \chi_{2} ; \mathbb{Z}_{p}\left[\chi_{1}, \chi_{2}\right]\right)$ such that

$$
C\left(\mathfrak{m}, E_{k}\left(\chi_{1}, \chi_{2}\right)\right)=\sum_{\mathfrak{a} \mid \mathfrak{m}} \chi_{1}(\mathfrak{a}) \chi_{2}\left(\frac{\mathfrak{m}}{\mathfrak{a}}\right) N(\mathfrak{a})^{k-1}
$$

for all nonzero integral ideals $\mathfrak{m}$ of $\mathcal{O}_{F}$ and

$$
C_{\lambda}\left(0, E_{k}\left(\chi_{1}, \chi_{2}\right)\right)= \begin{cases}2^{-d} \chi_{2}\left(t_{\lambda} \mathfrak{D}\right) L\left(1-k, \chi_{1} \chi_{2}^{-1}\right) & \text { if } \mathfrak{n}_{2}=1 \\ 0 & \text { otherwise. }\end{cases}
$$

Here $L(s, \chi)$ is the L-function associated to the character $\chi$ of conductor $\mathfrak{n}$, which is defined as the memromorphic continuation of the L-series

$$
L(s, \chi):=\sum_{(\mathfrak{a}, \mathfrak{n})=1} \chi(\mathfrak{a}) N(\mathfrak{a})^{-s}=\prod_{\mathfrak{p} \mathfrak{l} \mathfrak{n}}\left(1-\chi(\mathfrak{p}) N(\mathfrak{p})^{-s}\right)^{-1},
$$

which converges for $\operatorname{Re}(s)>1$. Moreover, we have

$$
S(\mathfrak{m}) \cdot E_{k}\left(\chi_{1}, \chi_{2}\right)=\chi_{1} \chi_{2}(\mathfrak{m}) \cdot E_{k}\left(\chi_{1}, \chi_{2}\right)
$$

for all integral ideals $\mathfrak{m}$ and

$$
T(\mathfrak{p}) \cdot E_{k}\left(\chi_{1}, \chi_{2}\right)=\left(\chi_{2}(\mathfrak{p})+\chi_{1}(\mathfrak{p}) N(\mathfrak{p})^{k-1}\right) \cdot E_{k}\left(\chi_{1}, \chi_{2}\right)
$$

for all prime ideals $\mathfrak{p}$. Therefore, $E_{k}\left(\chi_{1}, \chi_{2}\right)$ is a p-ordinary modular form if $\left(\mathfrak{n}_{2}, p\right)=1$. 


\subsection{Cusps of Hilbert modular varieties}

There are two parts in this subsection. The first part gives an adelic description of the cusps of Hilbert modular varieties so that we can compute constant terms of Eisenstein series at all cusps. The second part defines the Hecke action on the set of cusps and computes the ordinary projection of the set of cusps, which will be used to formulate Theorem 1.0.1.

We respectively denote by $B, T$, and $N$ the subgroup of upper-triangular matrices, the subgroup of diagonal matrices, and the unipotent subgroup of $\mathrm{GL}_{2}$. Recall that the set of the cusps for the Hilbert modular variety $Y_{K_{1}(\mathfrak{n})}$ is $C_{\mathfrak{n}}=\coprod_{\lambda=1}^{h_{F}^{+}} \Gamma_{1}\left(t_{\lambda} \mathfrak{D}, \mathfrak{n}\right) \backslash \mathbb{P}^{1}(F)$. An adelic description of cusps is as follows.

Lemma 3.4.1. Let the notation be as above. Then the map

$$
\begin{gathered}
C_{\mathfrak{n}} \longrightarrow \mathrm{GL}_{2}(F) N\left(\mathbb{A}_{F, f}\right) \backslash \mathrm{GL}_{2}\left(\mathbb{A}_{F, f}\right) \times \mathbb{P}^{1}(F) / K_{1}(\mathfrak{n}) \\
\Gamma_{1}\left(t_{\lambda} \mathfrak{D}, \mathfrak{n}\right)\left(\begin{array}{c}
a \\
c
\end{array}\right) \mapsto \mathrm{GL}_{2}(F) N\left(\mathbb{A}_{F, f}\right)\left(x_{\lambda}^{-1},\left(\begin{array}{c}
a \\
c
\end{array}\right)\right) K_{1}(\mathfrak{n})
\end{gathered}
$$

is bijective. Here $N\left(\mathbb{A}_{F, f}\right)$ (resp. $K_{1}(\mathfrak{n})$ ) acts on $\mathrm{GL}_{2}\left(\mathbb{A}_{F, f}\right) \times \mathbb{P}^{1}(F)$ by left multiplying (resp. right multiplying) on $\mathrm{GL}_{2}\left(\mathbb{A}_{F, f}\right)$. Recall that $x_{\lambda}$ was defined in Section 2.2.

Proof. We follow the argument in Proposition 3.2.3. We let $\Gamma_{\lambda}=\Gamma_{1}\left(t_{\lambda} \mathfrak{D}, \mathfrak{n}\right)$ for simplicity. Note that one can write $\mathbb{P}^{1}(F)=\mathrm{GL}_{2}(F) / B(F)=\mathrm{GL}_{2}(F)^{+} / B(F)^{+}$, where $\mathrm{GL}_{2}(F)^{+}$(resp. $\left.B(F)^{+}\right)$is the subgroup of $\mathrm{GL}_{2}(F)$ (resp. $B(F)$ ) consisting of all matrices with totally positive determinant. By (3.4), it is easy to see that the map (3.11) is surjective. To see the injectivity, we assume that $\Gamma_{\lambda} g B(F)^{+}$and $\Gamma_{\lambda} g^{\prime} B(F)^{+}$map to the same coset for $g, g^{\prime} \in \mathrm{GL}_{2}(F)^{+}$. Then there exist $\gamma_{1} \in \mathrm{GL}_{2}(F)$, $n \in N\left(\mathbb{A}_{F, f}\right), \beta \in B(F)^{+}$, and $\kappa_{1} \in K_{1}(\mathfrak{n})$ such that

$$
\left(x_{\lambda}^{-1}, g^{\prime}\right)=\gamma_{1} n\left(x_{\lambda}^{-1}, g \beta\right) \kappa_{1} .
$$


By strong approximation, we can write

$$
n=\left(\begin{array}{ll}
1 & a \\
0 & 1
\end{array}\right)=\gamma_{2} \kappa_{2}
$$

for some $\gamma_{2}=N(F)$ and $\kappa_{2}=\left(\begin{array}{ll}1 & k \\ 0 & 1\end{array}\right) \in \mathrm{GL}_{2}\left(\widehat{\mathcal{O}}_{F}\right)$ such that $k t_{\lambda} \delta \in \widehat{\mathcal{O}}_{F}$. Thus we have

$$
\left(x_{\lambda}^{-1}, g^{\prime}\right)=\gamma_{1} \gamma_{2}\left(\kappa_{2} x_{\lambda}^{-1} \kappa_{1}, g \beta\right)=\gamma_{1} \gamma_{2}\left(x_{\lambda}^{-1} \kappa_{2}^{\prime} \kappa_{1}, g \beta\right)
$$

for some $\kappa_{2}^{\prime} \in K_{1}(\mathfrak{n})$. Here the second equality is obtained by easy computation and the condition that $k t_{\lambda} \delta \in \widehat{\mathcal{O}}$. We set $\gamma=\gamma_{1} \gamma_{2}$ and $\kappa=\kappa_{2}^{\prime} \kappa_{1}$. From (3.12), we see that the finite part $\gamma_{f}$ of $\gamma$ and the infinite part $\gamma_{\infty}$ of $\gamma$ satisfy

$$
\gamma_{f}=x_{\lambda}^{-1} \kappa^{-1} x_{\lambda} \text { and } \gamma_{\infty}=g^{\prime} \beta^{-1} g^{-1}
$$

The former implies that $\gamma$ satisfies the congruence properties of $\Gamma_{\lambda}$, and the latter implies that $\gamma$ is totally positive. Hence, $\gamma \in \Gamma_{\lambda}$. This completes the proof.

We will always view the set of cusps $C_{\mathfrak{n}}$ for $K_{1}(\mathfrak{n})$ as the set of double cosets $\mathrm{GL}_{2}(F) N\left(\mathbb{A}_{F, f}\right) \backslash \mathrm{GL}_{2}\left(\mathbb{A}_{F, f}\right) \times \mathbb{P}^{1}(F) / K_{1}(\mathfrak{n})$. In order to state remaining results in this section, we will next simplify the set $C_{\mathfrak{n}}$.

As we have seen in the proof of Lemma 3.4.1, $\mathrm{GL}_{2}(F)$ acts on $\mathbb{P}^{1}(F)$ transitively, and the stabilizer of $\left(\begin{array}{l}1 \\ 0\end{array}\right)$ is $B(F)$. Therefore, we have

$$
\mathrm{GL}_{2}(F) N\left(\mathbb{A}_{F, f}\right) \backslash \mathrm{GL}_{2}\left(\mathbb{A}_{F, f}\right) \times \mathbb{P}^{1}(F) / K_{1}(\mathfrak{n})=B(F) N\left(\mathbb{A}_{F, f}\right) \backslash \mathrm{GL}_{2}\left(\mathbb{A}_{F, f}\right) \times\left\{\left(\begin{array}{l}
1 \\
0
\end{array}\right)\right\} / K_{1}(\mathfrak{n}) .
$$

By the Iwasawa decomposition (Lemma 2.2.2), one can decompose $\mathrm{GL}_{2}\left(\mathbb{A}_{F, f}\right)$ as

$$
\mathrm{GL}_{2}\left(\mathbb{A}_{F, f}\right)=B\left(\mathbb{A}_{F, f}\right) \mathrm{GL}_{2}\left(\widehat{\mathcal{O}}_{F}\right)
$$


and hence, we obtain the following equalities:

$$
\begin{aligned}
\mathrm{GL}_{2}(F) N\left(\mathbb{A}_{F, f}\right) \backslash \mathrm{GL}_{2}\left(\mathbb{A}_{F, f}\right) \times \mathbb{P}^{1}(F) / K_{1}(\mathfrak{n}) & =B(F) N\left(\mathbb{A}_{F, f}\right) \backslash \mathrm{GL}_{2}\left(\mathbb{A}_{F, f}\right) \times\left\{\left(\begin{array}{l}
1 \\
0
\end{array}\right)\right\} / K_{1}(\mathfrak{n}) \\
& =B(F) N\left(\mathbb{A}_{F, f}\right) \backslash \mathrm{GL}_{2}\left(\mathbb{A}_{F, f}\right) / K_{1}(\mathfrak{n}) \\
& =B(F) N\left(\mathbb{A}_{F, f}\right) \backslash B\left(\mathbb{A}_{F, f}\right) \mathrm{GL}_{2}\left(\widehat{\mathcal{O}}_{F}\right) / K_{1}(\mathfrak{n}) \\
& =B(F) N\left(\mathbb{A}_{F, f}\right) \backslash T\left(\mathbb{A}_{F, f}\right) \mathrm{GL}_{2}\left(\widehat{\mathcal{O}}_{F}\right) / K_{1}(\mathfrak{n}) .
\end{aligned}
$$

Here the last equality follows from the fact that $B\left(\mathbb{A}_{F, f}\right)=N\left(\mathbb{A}_{F, f}\right) T\left(\mathbb{A}_{F, f}\right)$.

From now on, we will always view the set $C_{\mathfrak{n}}$ of cusps as the set of double cosets $B(F) N\left(\mathbb{A}_{F, f}\right) \backslash T\left(\mathbb{A}_{F, f}\right) \mathrm{GL}_{2}\left(\widehat{\mathcal{O}}_{F}\right) / K_{1}(\mathfrak{n})$. Note that for any $\beta g \in C_{\mathfrak{n}}$ with $\beta \in T\left(\mathbb{A}_{F, f}\right)$ and $g \in \mathrm{GL}_{2}\left(\widehat{\mathcal{O}}_{F}\right)$, by right multiplying an element in $K_{1}(\mathfrak{n})$, we may assume $\operatorname{det} g=1$. We say that two cusps $c$ and $c^{\prime}$ are the same in $C_{\mathfrak{n}}$, denoted by $c \sim c^{\prime}$, if $c=\gamma c^{\prime} \kappa$ for some $\gamma \in B(F) N\left(\mathbb{A}_{F, f}\right)$ and $\kappa \in K_{1}(\mathfrak{n})$.

For any subset $C$ of $C_{\mathfrak{n}}$, we take $C^{*}=\left\{I_{[c]} \mid c \in C\right\}$ to be the set of indicator functions on $C$. The indicator function $I_{[c]}: C_{\mathfrak{n}} \longrightarrow\{0,1\}$ is defined by

$$
I_{[c]}\left(c^{\prime}\right)= \begin{cases}1 & c \sim c^{\prime} \\ 0 & \text { otherwise. }\end{cases}
$$

The Hecke actions on $C_{\mathfrak{n}}$ and on $C_{\mathfrak{n}}^{*}$ are defined as follows. Recall that for each prime ideal $\mathfrak{p}$, one has coset decompositions

$$
K_{1}(\mathfrak{n})\left(\begin{array}{cc}
\varpi_{\mathfrak{p}} & 0 \\
0 & 1
\end{array}\right) K_{1}(\mathfrak{n})=\coprod_{i} \gamma_{i} K_{1}(\mathfrak{n})=\coprod_{j} K_{1}(\mathfrak{n}) \beta_{j}
$$

for some $\gamma_{i}$ and $\beta_{j}$ in $\mathrm{GL}_{2}\left(\mathbb{A}_{F, f}\right)$. The operator $T(\mathfrak{p})$ acts on $C_{\mathfrak{n}}$ by

$$
T(\mathfrak{p}) \cdot c=\sum_{i} c \gamma_{i}
$$


and acts on $C_{\mathfrak{n}}^{*}$ by

$$
T(\mathfrak{p}) \cdot I_{[c]}=\sum_{j} I_{\left[c \beta_{j}^{-1}\right]}
$$

for all prime ideals $\mathfrak{p}$. We also have the operator $T^{*}(\mathfrak{p})$ that acts on $C_{\mathfrak{n}}$ by

$$
T^{*}(\mathfrak{p}) \cdot c=\sum_{j} c \beta_{j}^{-1}
$$

This operator will only be used in the proof of Corollary 3.4.3. It is easy to see that the homomorphism

$$
\mathbb{Z}_{p}\left[C_{\mathfrak{n}}^{*}\right] \longrightarrow \mathbb{Z}_{p}\left[C_{\mathfrak{n}}\right] ; \sum_{c \in C_{\mathfrak{n}}} a_{c} I_{[c]} \mapsto \sum_{c \in C_{\mathfrak{n}}} a_{c} c
$$

is an isomorphism of abelian groups which commutes with the $T(\mathfrak{p})$-action on the left and the $T^{*}(\mathfrak{p})$ action on the right for all prime ideals $\mathfrak{p}$.

Now we consider two subsets of cusps for the congruence subgroup $K_{1}\left(\mathfrak{n} p^{r}\right)$ for $r \in \mathbb{Z}_{>0}$. Let

$$
D_{r}=\left\{\left(\begin{array}{cc}
b_{1} & 0 \\
0 & b_{2}
\end{array}\right)\left(\begin{array}{cc}
a & b \\
c & d
\end{array}\right) \in T\left(\mathbb{A}_{F, f}\right) \mathrm{GL}_{2}\left(\widehat{\mathcal{O}}_{F}\right) \mid a d-b c=1 \text { and } \operatorname{val}_{\mathfrak{p}}(c)>0 \text { for all } \mathfrak{p} \mid p\right\}
$$

and

$$
\mathbf{D}_{r}=\left\{\left(\begin{array}{cc}
b_{1} & 0 \\
0 & b_{2}
\end{array}\right)\left(\begin{array}{cc}
a & b \\
c & d
\end{array}\right) \in T\left(\mathbb{A}_{F, f}\right) \mathrm{GL}_{2}\left(\widehat{\mathcal{O}}_{F}\right) \mid a d-b c=1 \text { and } \operatorname{val}_{\mathfrak{p}}(c)<r \text { for all } \mathfrak{p} \mid p\right\}
$$

be subsets of $\mathrm{GL}_{2}\left(\mathbb{A}_{F, f}\right)$, and let

$$
\overline{D_{r}}=B(F) N\left(\mathbb{A}_{F, f}\right) \backslash D_{r} / K_{1}\left(\mathfrak{n} p^{r}\right)
$$

and

$$
\overline{\mathbf{D}_{r}}=B(F) N\left(\mathbb{A}_{F, f}\right) \backslash \mathbf{D}_{r} / K_{1}\left(\mathfrak{n} p^{r}\right)
$$

be subsets of the set of double $\operatorname{cosets} C_{\mathfrak{n} p^{r}}$.

Theorem 3.4.2. Let the notation be as above. Assume that $p$ is unramified in $F$. Then 
1. $T(\mathfrak{p})^{n} \cdot \mathbb{Z}_{p}\left[\overline{D_{r}}\right] \subset \mathbb{Z}_{p}\left[\overline{D_{r}}\right]$ for all $\mathfrak{p} \mid p$ and for all $n \in \mathbb{N}$,

2. $e \cdot \mathbb{Z}_{p}\left[\overline{D_{r}}\right]=0$,

3. $e \cdot \mathbb{Z}_{p}\left[C_{\mathfrak{n} p^{r}}\right] \simeq \mathbb{Z}_{p}\left[C_{\mathfrak{n} p^{r}}\right] / \mathbb{Z}_{p}\left[\overline{D_{r}}\right]$,

Proof. Given any $\delta \in D_{r}$ and any $\mathfrak{p} \mid p$, we are going to compute $T(\mathfrak{p})^{n} \cdot \delta$ in $\mathbb{Z}_{p}\left[C_{\mathfrak{n} p^{r}}\right]$ for $n \in \mathbb{N}$. We first recall that for each $\mathfrak{p} \mid p$, we have

$$
K_{1}\left(\mathfrak{n} p^{r}\right)\left(\begin{array}{cc}
\varpi_{\mathfrak{p}} & 0 \\
0 & 1
\end{array}\right) K_{1}\left(\mathfrak{n} p^{r}\right)=\coprod_{u \in \mathcal{O}_{\mathfrak{p}} / \varpi_{\mathfrak{p}}}\left(\begin{array}{cc}
\varpi_{\mathfrak{p}} & u \\
0 & 1
\end{array}\right) K_{1}\left(\mathfrak{n} p^{r}\right)
$$

The following computation is at the place $\mathfrak{p}$ which is sufficient since the action of the Hecke operator $T(\mathfrak{p})$ is trivial at all places other than $\mathfrak{p}$. For simplicity, we write $\varpi_{\mathfrak{p}}$ as $\varpi$ and write val $\mathrm{p}_{\mathfrak{p}}$ as val. We write $\delta \in D_{r}$ as $\delta=\beta g=\left(\begin{array}{cc}b_{1} & 0 \\ 0 & b_{2}\end{array}\right)\left(\begin{array}{ll}a & b \\ c & d\end{array}\right)$, where $\beta \in T\left(F_{\mathfrak{p}}\right)$ and $g \in \mathrm{GL}_{2}\left(\mathcal{O}_{\mathfrak{p}}\right)$ with det $g=1$. Then we have

$$
\begin{aligned}
T(\mathfrak{p})^{n} \cdot \delta & =\sum_{u \in \mathcal{O}_{\mathfrak{p}} / \varpi^{n} \mathcal{O}_{\mathfrak{p}}}\left(\begin{array}{cc}
b_{1} & 0 \\
0 & b_{2}
\end{array}\right)\left(\begin{array}{ll}
a & b \\
c & d
\end{array}\right)\left(\begin{array}{cc}
\varpi^{n} & u \\
0 & 1
\end{array}\right) \\
& =\sum_{u \in \mathcal{O}_{\mathfrak{p}} / \varpi^{n} \mathcal{O}_{\mathfrak{p}}}\left(\begin{array}{cc}
b_{1} \varpi^{n} & 0 \\
0 & b_{2}
\end{array}\right)\left(\begin{array}{cc}
a & \varpi^{-n}(a u+b) \\
c \varpi^{n} & c u+d
\end{array}\right) .
\end{aligned}
$$

Since $\operatorname{val}(c)>0$, we know that $d$ and $c u+d$ are in $\mathcal{O}_{\mathfrak{p}}^{\times}$. Note that $\operatorname{det}\left(\begin{array}{c}a \varpi^{-n}(a u+b) \\ c \varpi^{n} c u+d\end{array}\right)=1$. By the Iwasawa decomposition, our formula for $T(\mathfrak{p})^{n} \cdot \delta$ is the same as

$$
\sum_{u \in \mathcal{O}_{\mathfrak{p}} / \varpi^{n} \mathcal{O}_{\mathfrak{p}}}\left(\begin{array}{cc}
b_{1} \varpi^{n} & 0 \\
0 & b_{2}
\end{array}\right)\left(\begin{array}{cc}
(c u+d)^{-1} & \varpi^{-n}(a u+b) \\
0 & c u+d
\end{array}\right)\left(\begin{array}{cc}
1 & 0 \\
c \varpi^{n}(c u+d)^{-1} & 1
\end{array}\right) .
$$

One can write this as

$$
\sum_{u \in \mathcal{O}_{\mathfrak{p}} / \varpi^{n} \mathcal{O}_{\mathfrak{p}}} \gamma_{u}\left(\begin{array}{cc}
b_{1} \varpi^{n} & 0 \\
0 & b_{2}
\end{array}\right)\left(\begin{array}{cc}
(c u+d)^{-1} & 0 \\
0 & c u+d
\end{array}\right)\left(\begin{array}{cc}
1 & 0 \\
c \varpi^{n}(c u+d)^{-1} & 1
\end{array}\right)
$$

for some $\gamma_{u} \in N\left(\mathbb{A}_{F, f}\right)$ for all $u \in \mathcal{O}_{\mathfrak{p}} / \varpi^{n} \mathcal{O}_{\mathfrak{p}}$. As formal sums of double cosets, this equals

$$
\sum_{u \in \mathcal{O}_{\mathfrak{p}} / \varpi^{n} \mathcal{O}_{\mathfrak{p}}}\left(\begin{array}{cc}
b_{1} \varpi^{n} & 0 \\
0 & b_{2}
\end{array}\right)\left(\begin{array}{cc}
(c u+d)^{-1} & 0 \\
0 & c u+d
\end{array}\right)\left(\begin{array}{cc}
1 & 0 \\
c \varpi^{n}(c u+d)^{-1} & 1
\end{array}\right) .
$$


This yields

$$
T(\mathfrak{p})^{n} \cdot \delta=\sum_{u \in \mathcal{O}_{\mathfrak{p}} / \varpi^{n} \mathcal{O}_{\mathfrak{p}}}\left(\begin{array}{cc}
b_{1} \varpi^{n} & 0 \\
0 & b_{2}
\end{array}\right)\left(\begin{array}{cc}
(c u+d)^{-1} & 0 \\
c \varpi^{n} & c u+d
\end{array}\right) .
$$

Hence the element $T(\mathfrak{p})^{n} \cdot \delta$ is in $\mathbb{Z}_{p}\left[\overline{D_{r}}\right]$. Thus the first assertion follows.

For the second assertion, we claim that the element (3.14) is equivalent to

$$
N(\varpi)^{n-r} \sum_{j \in \mathcal{O}_{\mathfrak{p}} / \varpi^{r} \mathcal{O}_{\mathfrak{p}}}\left(\begin{array}{cc}
b_{1} \varpi^{n} & 0 \\
0 & b_{2}
\end{array}\right)\left(\begin{array}{cc}
(c j+d)^{-1} & 0 \\
0 & c j+d
\end{array}\right)
$$

for all $n \geq r$ and note that the sequence of such elements converges to 0 under the $p$-adic topology as $n \longrightarrow \infty$. We write $u=j+\varpi^{r} s$, where $j=\sum_{i=0}^{r-1} \alpha_{i} \varpi^{i}$ for $\alpha_{i} \in \mathcal{O}_{\mathfrak{p}}^{\times}$and $s \in \mathcal{O}_{\mathfrak{p}}$. To prove the claim, it suffices to show that for each $u \in \mathcal{O}_{\mathfrak{p}} / \varpi^{n} \mathcal{O}_{\mathfrak{p}}$, there exist $X, Y \in \mathcal{O}_{\mathfrak{p}}$ such that

$$
\left(\begin{array}{cc}
(c u+d)^{-1} & 0 \\
c \varpi^{n} & c u+d
\end{array}\right)\left(\begin{array}{cc}
\left(1+\varpi^{r} Y\right)^{-1} & 0 \\
\varpi^{r} X & 1+\varpi^{r} Y
\end{array}\right)=\left(\begin{array}{cc}
(c j+d)^{-1} & 0 \\
0 & c j+d
\end{array}\right) .
$$

To find $Y$, it suffices to solve the equation

$$
(c u+d)\left(1+\varpi^{r} Y\right)=c j+d
$$

integrally, which is possible since $c u+d \in \mathcal{O}_{\mathfrak{p}}^{\times}$and since if two units are congruent modulo $\varpi^{r}$ then they differ by multiplication by an element of $1+\varpi^{r} \mathcal{O}_{\mathfrak{p}}$. To find $X$, we solve the equation

$$
c \varpi^{n}\left(1+\varpi^{r} Y\right)^{-1}+\varpi^{r} X(c u+d)=0 .
$$

It is easy to see that the solution is

$$
X=-c \varpi^{n-r}\left(1+\varpi^{r} Y\right)^{-1}(c u+d)^{-1} \in \mathcal{O}_{\mathfrak{p}} .
$$

For the last assertion, we consider the sequence

$$
0 \longrightarrow \operatorname{ker} \longrightarrow \mathbb{Z}_{p}\left[C_{\mathfrak{n} p^{r}}\right] \longrightarrow e \cdot \mathbb{Z}_{p}\left[C_{\mathfrak{n} p^{r}}\right] \longrightarrow 0
$$


It is enough to show that ker $=\mathbb{Z}_{p}\left[\overline{D_{r}}\right]$. It follows from the second assertion that $\mathbb{Z}_{p}\left[\overline{D_{r}}\right] \subset$ ker. Thus, it remains to show that for any $\gamma=\sum_{\delta \in C_{\mathfrak{n} p^{r}}-\overline{D_{r}}} a_{\delta} \delta \notin \mathbb{Z}_{p}\left[\overline{D_{r}}\right]$, we have

$$
e \cdot \gamma \neq 0 \in e \cdot \mathbb{Z}_{p}\left[C_{\mathfrak{n} p^{r}}\right]
$$

Since $T(\mathfrak{p}) \cdot \gamma=\sum_{\delta \in C_{\mathfrak{n} p^{r}}-\overline{D_{r}}} a_{c}(T(\mathfrak{p}) \cdot \delta)$, it suffices to show two things: one is that $T(\mathfrak{p})^{n} \cdot \delta$ is a constant times a single nonzero cusp in $e \cdot \mathbb{Z}_{p}\left[C_{\mathfrak{n} p^{r}}\right]$ and is not in $\bar{D}_{r}$ for all $n$ big enough. The other is that for all $\delta_{1}, \delta_{2} \notin \bar{D}_{r}$ with $\delta_{1} \nsim \delta_{2}, T(\mathfrak{p})^{n} \cdot \delta_{1} \nsim T(\mathfrak{p})^{n} \cdot \delta_{2}$ for all $n$ big enough, for some $\mathfrak{p} \mid p$. Let $\beta g=\left(\begin{array}{cc}b_{1} & 0 \\ 0 & b_{2}\end{array}\right)\left(\begin{array}{ll}a & b \\ c & d\end{array}\right) \notin D_{r}$, i.e., $\operatorname{val}_{\mathfrak{p}}(c)=0$ for some $\mathfrak{p} \mid p$. We fix such a prime $\mathfrak{p}$ and write $\operatorname{val}_{\mathfrak{p}}=$ val for simplicity. We first show that $e \cdot \beta g \neq 0$ by explicitly computing $T(\mathfrak{p})^{n} \cdot \beta g$ for all $n \geq r$. For each $n \geq r$, right multiplying the matrix $g$ by the matrix $\left(\begin{array}{c}1 \\ 0 \\ \varpi^{n}-c^{-1} d \\ 1\end{array}\right) \in K_{1, \mathfrak{p}}\left(\mathfrak{n} p^{r}\right)$ if necessary, we may assume $\operatorname{val}(d) \geq n$. It is easy to see that $T(\mathfrak{p})^{n} \cdot\left(\begin{array}{cc}b_{1} & 0 \\ 0 & b_{2}\end{array}\right)\left(\begin{array}{ll}a & b \\ c & d\end{array}\right)$ is

$$
\sum_{u \in \mathcal{O}_{\mathfrak{p}} / \varpi^{n} \mathcal{O}_{\mathfrak{p}}}\left(\begin{array}{cc}
b_{1} & 0 \\
0 & b_{2}
\end{array}\right)\left(\begin{array}{ll}
\varpi^{n} a & a u+b \\
\varpi^{n} c & c u+d
\end{array}\right)
$$

If $u \neq 0$, then $\operatorname{val}(c u+d)<n$. By the Iwasawa decomposition, we have

$$
\left(\begin{array}{cc}
b_{1} & 0 \\
0 & b_{2}
\end{array}\right)\left(\begin{array}{ll}
\varpi^{n} a & a u+b \\
\varpi^{n} c & c u+d
\end{array}\right) \sim\left(\begin{array}{cc}
b_{1} \varpi^{n} & 0 \\
0 & b_{2}
\end{array}\right)\left(\begin{array}{cc}
a(c u+d)^{-1} & 0 \\
\varpi^{n} c & c u+d
\end{array}\right) \in D_{r}
$$

which is 0 in $e \cdot \mathbb{Z}_{p}\left[C_{\mathfrak{n} p^{r}}\right]$. If $u=0$, then we have

$$
\left(\begin{array}{cc}
b_{1} & 0 \\
0 & b_{2}
\end{array}\right)\left(\begin{array}{cc}
\varpi^{n} a & b \\
\varpi^{n} c & d
\end{array}\right)=\left(\begin{array}{cc}
b_{1} & 0 \\
0 & b_{2} \varpi^{n}
\end{array}\right)\left(\begin{array}{cc}
\varpi^{n} a & b \\
c & d \varpi^{-n}
\end{array}\right)
$$

Since $\operatorname{val}(c)=0$ and $\operatorname{val}_{\mathfrak{p}}(d) \geq n$, this element is in $B\left(F_{\mathfrak{p}}\right) \mathrm{GL}_{2}\left(\mathcal{O}_{\mathfrak{p}}\right)$ and is not in $\overline{D_{r}}$.

Next, we claim that if $\gamma_{1}, \gamma_{2} \in C_{\mathfrak{n} p^{r}}-\bar{D}_{r}$ are not equivalent, then $T(\mathfrak{p})^{n} \cdot \gamma_{1}, T(\mathfrak{p})^{n} \cdot \gamma_{2} \in e \cdot \mathbb{Z}_{p}\left[C_{\mathfrak{n} p^{r}}\right]$ are not equivalent for some $\mathfrak{p} \mid p$ and for all $n$ big enough. We now write $\gamma_{i}=\beta_{i} g_{i}=\left(\begin{array}{cc}\alpha_{i} & 0 \\ 0 & \alpha_{i}^{\prime}\end{array}\right)\left(\begin{array}{cc}a_{i} & b_{i} \\ c_{i} & d_{i}\end{array}\right)$ with $\operatorname{det} g_{i}=1$ for $i=1,2$. Since $\gamma_{1} \notin \bar{D}_{r}$, there exists a prime ideal $\mathfrak{p}$ such that $\operatorname{val}_{\mathfrak{p}}\left(c_{1}\right)=0$. If $\operatorname{val}_{\mathfrak{p}}\left(c_{2}\right)=0$, then it follows from the above computations (3.15), (3.16), and (3.17) that for all $n \geq r$ 
and for $i=1,2$, we have

$T(\mathfrak{p})^{n} \cdot \gamma_{i} \sim\left(\begin{array}{cc}\alpha_{i} & 0 \\ 0 & \alpha_{i}^{\prime} \varpi^{n}\end{array}\right)\left(\begin{array}{cc}\varpi^{n} a_{i} & b_{i} \\ c_{i} & d_{i} \varpi^{-n}\end{array}\right) \sim\left(\begin{array}{cc}\alpha_{i} & 0 \\ 0 & \alpha_{i}^{\prime} \varpi^{n}\end{array}\right)\left(\begin{array}{cc}c_{i}^{-1} & 0 \\ 0 & c_{i}\end{array}\right)\left(\begin{array}{cc}1 & 0 \\ 1 & 1\end{array}\right) \in e \cdot \mathbb{Z}_{p}\left[C_{\mathfrak{n} p^{r}}\right]$.

Here the second equivalence is obtained by (2.2). By (2.2) again, we see that for $i=1,2$, we have

$$
\gamma_{i} \sim\left(\begin{array}{cc}
\alpha_{i} & 0 \\
0 & \alpha_{i}^{\prime}
\end{array}\right)\left(\begin{array}{cc}
c_{i}^{-1} & 0 \\
0 & c_{i}
\end{array}\right)\left(\begin{array}{ll}
1 & 0 \\
1 & 1
\end{array}\right)
$$

Since $\gamma_{1}$ and $\gamma_{2}$ are not equivalent, $T(\mathfrak{p})^{n} \cdot \gamma_{1}$ and $T(\mathfrak{p})^{n} \cdot \gamma_{2}$ are also not equivalent for all $n \geq r$. Now we suppose that $\operatorname{val}_{\mathfrak{p}}\left(c_{2}\right) \neq 0$. Then, by the argument of the first assertion, it is clear that the claim holds.

Corollary 3.4.3. Let the notation and the assumption as in Theorem 3.4.2. Then we have

$$
e \cdot \mathbb{Z}_{p}\left[C_{\mathfrak{n} p^{r}}^{*}\right]=\mathbb{Z}_{p}\left[C_{\mathfrak{n} p^{r}}^{*}\right] / \mathbb{Z}_{p}\left[{\overline{\mathbf{D}_{r}}}^{*}\right]
$$

Proof. By (3.13), to show the assertion, it is equivalent to show that

$$
e^{*} \cdot \mathbb{Z}_{p}\left[C_{\mathfrak{n} p^{r}}\right]=\mathbb{Z}_{p}\left[C_{\mathfrak{n} p^{r}}\right] / \mathbb{Z}_{p}\left[\overline{\mathbf{D}_{r}}\right]
$$

where $e^{*}=\lim _{n \rightarrow \infty} \prod_{\mathfrak{p} \mid p} T^{*}(\mathfrak{p})^{n !}$. We first observe the relationship between the action of $e$ and the action of $e^{*}$ on $\mathbb{Z}_{p}\left[C_{\mathfrak{n} p^{r}}\right]$. Note that

$$
K_{1}\left(\mathfrak{n} p^{r}\right)\left(\begin{array}{cc}
\varpi & 0 \\
0 & 1
\end{array}\right) K_{1}\left(\mathfrak{n} p^{r}\right)=\coprod_{u \in \mathcal{O}_{\mathfrak{p}} / \varpi} K_{1}\left(\mathfrak{n} p^{r}\right) \tau_{\mathfrak{p}}^{-1}\left(\begin{array}{cc}
\varpi & u \\
0 & 1
\end{array}\right)^{\iota} \tau_{\mathfrak{p}}
$$

where $\tau_{\mathfrak{p}}=\left(\begin{array}{cc}0 & -1 \\ \varpi^{\operatorname{val}_{\mathfrak{p}}(p) r} & 0\end{array}\right) \in \mathrm{GL}_{2}\left(F_{\mathfrak{p}}\right)$. In fact, we have $\tau_{\mathfrak{p}}=\left(\begin{array}{cc}0 & -1 \\ \varpi^{r} & 0\end{array}\right)$, since $p$ is unramified in $F$. We will also view $\tau_{\mathfrak{p}}$ as an element in $\mathrm{GL}_{2}\left(\mathbb{A}_{F, f}\right)$ whose entries at finite places of $F$ other than $\mathfrak{p}$ are identity matrices. The operator $\iota$ is the standard involution on $\mathrm{GL}_{2}$ defined as $\left(\begin{array}{ll}a & b \\ c & d\end{array}\right)^{\iota}=\left(\begin{array}{cc}d & -b \\ -c & a\end{array}\right)$. Note that one has $A A^{\iota}=\operatorname{det} A \cdot I_{2}$ for all $A \in \mathrm{GL}_{2}$, where $I_{2}$ is the identity matrix in $\mathrm{GL}_{2}$. It follows from this applied to $A=\left(\begin{array}{cc}\varpi & u \\ 0 & 1\end{array}\right)$ for some $u \in \mathcal{O}_{\mathfrak{p}} / \varpi \mathcal{O}_{\mathfrak{p}}$ and the definition of $T^{*}(\mathfrak{p})$ that

$$
T^{*}(\mathfrak{p}) \cdot c=\tau_{\mathfrak{p}}^{-1} T(\mathfrak{p}) \tau_{\mathfrak{p}}\left(\begin{array}{cc}
\varpi^{-1} & 0 \\
0 & \varpi^{-1}
\end{array}\right) \cdot c
$$


for all $c \in C_{\mathfrak{n} p^{r}}$. Here for an element $\gamma \in \mathrm{GL}_{2}\left(\mathbb{A}_{F, f}\right), \gamma \cdot c$ is defined as $c \gamma$ for all $c \in C_{\mathfrak{n} p^{r}}$. It is easy to see that $\left(\begin{array}{cc}\varpi^{-1} & 0 \\ 0 & \varpi^{-1}\end{array}\right) \cdot C_{\mathfrak{n} p^{r}}=C_{\mathfrak{n} p^{r}}$, so one obtains $T^{*}(\mathfrak{p}) \mathbb{Z}_{p}\left[C_{\mathfrak{n} p^{r}}\right]=\tau_{\mathfrak{p}}^{-1} T(\mathfrak{p}) \tau_{\mathfrak{p}} \mathbb{Z}_{p}\left[C_{\mathfrak{n} p^{r}}\right]$ and hence, $T^{*}(\mathfrak{p})^{n} \mathbb{Z}_{p}\left[C_{\mathfrak{n} p^{r}}\right]=\tau_{\mathfrak{p}}^{-1} T(\mathfrak{p})^{n} \tau_{\mathfrak{p}} \mathbb{Z}_{p}\left[C_{\mathfrak{n} p^{r}}\right]$ for all positive integers $n$. This yields

$$
e^{*} \cdot \mathbb{Z}_{p}\left[C_{\mathfrak{n} p^{r}}\right]=\tau_{p}^{-1} e \tau_{p} \cdot \mathbb{Z}_{p}\left[C_{\mathfrak{n} p^{r}}\right]
$$

where $\tau_{p}=\prod_{\mathfrak{p} \mid p} \tau_{\mathfrak{p}}$.

Next, we set $K=\prod_{v \nmid p} K_{1, v}\left(\mathfrak{n} p^{r}\right) \times \prod_{v \mid p} K_{v}^{1}\left(\mathfrak{n} p^{r}\right)$ and

$$
C_{\mathfrak{n} p^{r}}^{1}=B(F) N\left(\mathbb{A}_{F, f}\right) \backslash T\left(\mathbb{A}_{F, f}\right) \mathrm{GL}_{2}\left(\widehat{O}_{F}\right) / K
$$

Recall that the group $K_{v}^{1}\left(\mathfrak{n} p^{r}\right)$ was defined in Section 3.2. Then we have $\tau_{p}^{-1} \cdot \mathbb{Z}_{p}\left[C_{\mathfrak{n} p^{r}}\right]=\mathbb{Z}_{p}\left[C_{\mathfrak{n} p^{r}}^{1}\right]$ as $\tau_{p} \cdot c$ is a cusp for $\tau_{p}^{-1} K_{1}\left(\mathfrak{n} p^{r}\right) \tau_{p}=K$. Note that in the proof of Theorem 3.4.2, when we showed two cusps are equivalent by multiplying by matrices in $K_{1}(\mathfrak{n})$, those matrices are always in $K_{1}^{1}(\mathfrak{n})$. Therefore, by the same argument as in Theorem 3.4.2, we have

$$
e \cdot \mathbb{Z}_{p}\left[C_{\mathfrak{n} p^{r}}^{1}\right]=\mathbb{Z}_{p}\left[C_{\mathfrak{n} p^{r}}^{1}\right] / \mathbb{Z}_{p}\left[B(F) N\left(\mathbb{A}_{F, f}\right) \backslash D_{r} / K\right]
$$

Finally, we observe that $\tau_{\mathfrak{p}} \cdot D_{r}=\mathbf{D}_{r}$ for all $\mathfrak{p} \mid p$. To see this, we suppose that $\delta=\beta g=$ $\left(\begin{array}{cc}b_{1} & 0 \\ 0 & b_{2}\end{array}\right)\left(\begin{array}{ll}a & b \\ c & d\end{array}\right) \in D_{r}$ is given. We fix an arbitrary prime ideal $\mathfrak{p}$ of $F$ dividing $p$ and write $\operatorname{val}_{\mathfrak{p}}(c)=s>0$ If $s \geq r$, then we have

$$
\tau_{\mathfrak{p}} \cdot \delta=\beta\left(\begin{array}{cc}
b \varpi^{r} & -a \\
d \varpi^{r} & -c
\end{array}\right)=\left(\begin{array}{cc}
b_{1} & 0 \\
0 & b_{2} \varpi^{r}
\end{array}\right)\left(\begin{array}{cc}
b \varpi^{r} & -a \\
d & -c \varpi^{-r}
\end{array}\right) \in \mathbf{D}_{r}
$$

If $0<s<r$, by a similar computation we again have $\tau_{\mathfrak{p}} \cdot \delta \in \mathbf{D}_{r}$. Thus we see that

$$
\tau_{p} \cdot \mathbb{Z}_{p}\left[C_{\mathfrak{n} p^{r}}^{1}\right] / \mathbb{Z}_{p}\left[B(F) N\left(\mathbb{A}_{F, f}\right) \backslash D_{r} / K\right]=\mathbb{Z}_{p}\left[C_{\mathfrak{n} p^{r}}\right] / \mathbb{Z}_{p}\left[\overline{\mathbf{D}_{r}}\right]
$$


To sum up, we have shown that

$$
\begin{aligned}
e^{*} \mathbb{Z}_{p}\left[C_{\mathfrak{n} p^{r}}\right] & =\tau_{p} e \tau_{p}^{-1} \cdot \mathbb{Z}_{p}\left[C_{\mathfrak{n} p^{r}}\right] \\
& =\tau_{p} e \cdot \mathbb{Z}_{p}\left[C_{\mathfrak{n} p^{r}}^{1}\right] \\
& =\tau_{p} \cdot \mathbb{Z}_{p}\left[C_{\mathfrak{n} p^{r}}^{1}\right] / \mathbb{Z}_{p}\left[B(F) N\left(\mathbb{A}_{F, f}\right) \backslash D_{r} / K\right] \\
& =\mathbb{Z}_{p}\left[C_{\mathfrak{n} p^{r}}\right] / \mathbb{Z}_{p}\left[\overline{\mathbf{D}_{r}}\right] .
\end{aligned}
$$

The proof is complete.

Define a $\mathbb{Z}_{p}$-homomorphism $C_{0}: M_{k}\left(K_{1}(\mathfrak{n}) ; \mathbb{Z}_{p}\right) \longrightarrow \mathbb{Z}_{p}\left[C_{\mathfrak{n}}^{*}\right]$ as

$$
C_{0}(f)=\sum_{[g] \in C_{\mathfrak{n}}}\left(\int_{\mathbb{A}_{F} / F} f(n(x) g) d x\right) \cdot I_{[g]}
$$

where $n(x)=\left(\begin{array}{ll}1 & x \\ 0 & 1\end{array}\right)$ for all $x \in \mathbb{A}_{F}$, which is well-defined as $f$ is left $\mathrm{GL}_{2}(F)$-invariant. Moreover, it does not depend on the choice of the representatives of $C_{\mathfrak{n}}$. To see this, we observe that for any $\beta=\left(\begin{array}{ll}a & b \\ 0 & d\end{array}\right) \in B(F), n(t) \in N\left(\mathbb{A}_{F, f}\right)$, and $\kappa \in K_{1}(\mathfrak{n})$, by substitution and the fact that $f$ is right $K_{1}(\mathfrak{n})$-invariant, we have

$$
\int_{\mathbb{A}_{F} / F} f(n(x) \gamma n g \kappa) d x=|d / a|_{\mathbb{A}} \int_{\mathbb{A}_{F} / F} f(n(x) g) d x=\int_{\mathbb{A}_{F} / F} f(n(x) g) d x
$$

Here the last equality is obtained by the assumption that $a, d \in F$ and that $|d / a|_{\mathbb{A}}=\prod_{v}|b / a|_{v}=1$, where $v$ runs through all places of $F$.

Lemma 3.4.4. Let the notation be as above. Then the map $C_{0}$ commutes with the Hecke actions.

Proof. By the same argument as in [DS, Lemma 5.5.1], there exists a set of elements $\left\{\gamma_{i}\right\}$ in $\mathrm{GL}_{2}\left(\mathbb{A}_{F, f}\right)$ such that

$$
K_{1}(\mathfrak{n})\left(\begin{array}{rr}
\varpi_{\mathfrak{p}} & 0 \\
0 & 1
\end{array}\right) K_{1}(\mathfrak{n})=\coprod_{i} \gamma_{i} K_{1}(\mathfrak{n})=\coprod_{i} K_{1}(\mathfrak{n}) \gamma_{i}
$$


for all prime ideals $\mathfrak{p}$ of $F$. Then we have

$$
\begin{aligned}
C_{0}(T(\mathfrak{p}) \cdot f) & =\sum_{[g] \in C_{\mathfrak{n}}} \sum_{i}\left(\int_{\mathbb{A}_{F} / F} f\left(n(x) g \gamma_{i}\right) d x\right) I_{[g]} \\
& =\sum_{i} \sum_{[g] \in C_{\mathfrak{n}}}\left(\int_{\mathbb{A}_{F} / F} f\left(n(x) g \gamma_{i}\right) d x\right) I_{[g]} \\
& =\sum_{i} \sum_{[G] \in C_{\mathfrak{n}}}\left(\int_{\mathbb{A}_{F} / F} f(n(x) G) d x\right) I_{\left[G \gamma_{i}^{-1}\right]} \\
& =T(\mathfrak{p}) C_{0}(f) .
\end{aligned}
$$

The assertion follows. 


\section{CHAPTER 4}

Automorphic forms

The main goal of this section is to construct the Eisenstein series in Proposition 3.3.3 as automorphic forms and to compute their constant terms at different cusps. Throughout this section, we fix an additive character $\psi=\otimes_{v} \psi_{v}$ on $\mathbb{A}_{F}$ defined as $\psi_{v}(x)=e^{2 \pi i x}$ if $v \mid \infty$ and $\psi_{v}(x)=e^{-2 \pi i\left[\operatorname{Tr}_{F_{v}} / \mathbb{Q}_{p}(x)\right]_{p}}$ if $v \mid p$, where $[x]_{p}$ is the fractional part of $x$ for $x \in \mathbb{Q}_{p}$. Here $v$ runs through all places of $F$. We also fix a pair of primitive narrow ray class characters $\chi=\left(\chi_{1}, \chi_{2}\right)$ of conductor $\mathfrak{n}_{1}$ and $\mathfrak{n}_{2}$, respectively. Let $\mathfrak{n}=\mathfrak{n}_{1} \mathfrak{n}_{2}$. For our application in Chapter 7 , we assume that $\mathfrak{n}$ is prime to $\mathfrak{D}$. Note that everything in this chapter can be done without this assumption.

For $i=1,2$, we write $\mathfrak{n}_{i}=\prod_{\mathfrak{p} \mid \mathfrak{n}_{i}} \mathfrak{p}^{e_{\mathfrak{p}, i}}$, and the finite part $\chi_{i, f}$ of $\chi_{i}$ can be decomposed as

$$
\chi_{i, f}=\prod_{\mathfrak{p} \mid \mathfrak{n}_{i}} \chi_{i}^{(\mathfrak{p})}: \prod_{\mathfrak{p} \mid \mathfrak{n}_{i}}\left(\mathcal{O} / \mathfrak{p}^{e_{\mathfrak{p}, i}}\right)^{\times} \longrightarrow \mathbb{C}^{\times} .
$$

We also denote by $\chi_{i}=\prod_{v} \chi_{i, v}$ their corresponding Hecke characters. Here $v$ runs through all places of $F$.

\subsection{Non-archimedean sections}

We fix a finite place $v$ of $F$. Let $\mathcal{O}_{v}$ be the ring of integers of $F_{v}$. We fix an uniformizer $\varpi_{v}$ in $\mathcal{O}_{v}$, and let $q_{v}$ be the cardinality of the residue field of $\mathcal{O}_{v}$. Let $\mathfrak{p}_{v}$ be the maximal ideal of $\mathcal{O}_{v}$. Sometimes we write $F_{\mathfrak{p}}$ for $F_{v}$ and $\mathcal{O}_{\mathfrak{p}}$ for $\mathcal{O}_{v}$. We will omit $v$ from $\varpi_{v}, q_{v}$, and $\mathfrak{p}_{v}$ if there is no confusion. Let $I\left(\chi_{1, v}|\cdot|{ }_{v}^{s},\left.\chi_{2, v}|\cdot|\right|_{v} ^{-s}\right)$ be the set of all functions $f_{s}: \mathrm{GL}_{2}\left(F_{v}\right) \longrightarrow \mathbb{C}$ satisfying

$$
f_{s}\left(\left(\begin{array}{cc}
a & b \\
0 & d
\end{array}\right) g\right)=\chi_{1, v}(a) \chi_{2, v}(d)\left|\frac{a}{d}\right|_{v}^{s+\frac{1}{2}} f_{s}(g)
$$


for $a, b, d \in F_{v}, g \in \mathrm{GL}_{2}\left(F_{v}\right)$, and $s \in \mathbb{C}$. Let $f_{\chi, s, v} \in I\left(\chi_{1, v}|\cdot|{ }_{v}^{s}, \chi_{2, v}|\cdot|{ }_{v}^{-s}\right)$ be the new section as in [Cas, Theorem 1.1] or see [Sch, Proposition 2.1.2]. We have seen from Lemma 2.2.3 that one can decompose $\mathrm{GL}_{2}\left(\mathcal{O}_{v}\right)$ as

$$
\mathrm{GL}_{2}\left(\mathcal{O}_{v}\right)=\bigcup_{i=0}^{N} B\left(\mathcal{O}_{v}\right) \gamma_{i} K_{1, v}\left(\mathfrak{p}^{N}\right),
$$

where $N=e_{\mathfrak{p}, 1}+e_{\mathfrak{p}, 2}$, and $\gamma_{i}$ is $\left(\begin{array}{cc}1 & 0 \\ \varpi_{v}^{i} & 1\end{array}\right)$ if $0 \leq i \leq N-1$ and is the identity matrix if $i=N$. This decomposition will be used constantly in this section. We now recall the definition of $f_{\chi, s, v}$. The section $f_{\chi, s, v}$ is the unique function on $\mathrm{GL}_{2}\left(F_{v}\right)$ satisfying (4.1) and the following properties:

1. for $g \in \mathrm{GL}_{2}\left(F_{v}\right)$, we have $f_{\chi, s, v}\left(g\left(\begin{array}{ll}a & b \\ c & d\end{array}\right)\right)=f_{\chi, s, v}(g) \chi_{1, v} \chi_{2, v}(d)$ for $\left(\begin{array}{ll}a & b \\ c & d\end{array}\right) \in K_{0, v}(\mathfrak{n})$,

2. for $g \in \mathrm{GL}_{2}\left(\mathcal{O}_{v}\right)$, we have $f_{\chi, s, v}(g)= \begin{cases}\chi_{1, v}\left(\varpi^{-e_{\mathfrak{p}, 2}}\right) \chi_{1, v}(a) \chi_{2, v}(d) & \text { if } g \in\left(\begin{array}{ll}a & b \\ 0 & d\end{array}\right) \gamma_{e_{\mathfrak{p}, 2}} K_{1, v}(\mathfrak{n}) \\ 0 & \text { otherwise. }\end{cases}$ Here $\left(\begin{array}{ll}a & b \\ 0 & d\end{array}\right) \in B\left(\mathcal{O}_{v}\right)$. Note that the factor $\chi_{1, v}\left(\varpi^{-e_{p}, 2}\right)$ in the last condition makes $f_{\chi, s, v}$ be independent of the choice of a uniformizer.

The following lemma was proved in [Sch, Proposition 2.1.2].

Lemma 4.1.1. Let $f_{\chi, s, v}$ be defined as above.

1. If $\chi_{1, v}$ and $\chi_{2, v}$ are ramified, then

$$
f_{\chi, s, v}\left(\left(\begin{array}{cc}
1 & 0 \\
x & 1
\end{array}\right)\right)= \begin{cases}\chi_{1, v}^{-1}(x) & \text { if } \operatorname{val}(x)=e_{\mathfrak{p}, 2} \\
0 & \text { if } \operatorname{val}(x) \neq e_{\mathfrak{p}, 2}\end{cases}
$$

2. If $\chi_{1, v}$ is unramified and $\chi_{2, v}$ is ramified, then

$$
f_{\chi, s, v}\left(\left(\begin{array}{cc}
1 & 0 \\
x & 1
\end{array}\right)\right)= \begin{cases}\chi_{1, v}^{-1}\left(\varpi^{e_{\mathfrak{p}, 2}}\right) & \text { if } \operatorname{val}(x) \geq e_{\mathfrak{p}, 2} \\
0 & \text { if } \operatorname{val}(x)<e_{\mathfrak{p}, 2}\end{cases}
$$


3. If $\chi_{1, v}$ is ramified and $\chi_{2, v}$ is unramified, then

$$
f_{\chi, s, v}\left(\left(\begin{array}{cc}
1 & 0 \\
x & 1
\end{array}\right)\right)= \begin{cases}\chi_{1, v}^{-1} \chi_{2, v}(x)|x|_{v}^{-(2 s+1)} & \text { if } \operatorname{val}(x) \leq 0 \\
0 & \text { if } \operatorname{val}(x)>0\end{cases}
$$

4. If $\chi_{1, v}$ and $\chi_{2, v}$ are unramified, then

$$
f_{\chi, s, v}\left(\left(\begin{array}{cc}
1 & 0 \\
x & 1
\end{array}\right)\right)= \begin{cases}\chi_{1, v}^{-1} \chi_{2, v}(x)|x|_{v}^{-(2 s+1)} & \text { if } \operatorname{val}(x)<0 \\
1 & \text { if } \operatorname{val}(x) \geq 0\end{cases}
$$

ProOF. Since the arguments for the four assertions are basically the same, we only give a proof of the last assertion here. Assume that both $\chi_{1, v}$ and $\chi_{2, v}$ are unramified. Note that we have $\mathrm{GL}_{2}\left(\mathcal{O}_{v}\right)=K_{1, v}(\mathfrak{n})$ in this case. Thus it follows from the definition of $f_{\chi, s, v}$ that $f_{\chi, s, v}\left(\left(\begin{array}{ll}1 & 0 \\ x & 1\end{array}\right)\right)=1$ for all $x \in \mathcal{O}_{v}$. For $x \in F_{v}-\mathcal{O}_{v}$, by the Iwasawa decomposition and by (2.2), we have

$$
\begin{aligned}
\left(\begin{array}{ll}
1 & 0 \\
x & 1
\end{array}\right) & =\left(\begin{array}{cc}
-x^{-1} & 1 \\
0 & x
\end{array}\right)\left(\begin{array}{cc}
0 & 1 \\
1 & x^{-1}
\end{array}\right) \\
& =\left(\begin{array}{cc}
-x^{-1} & 1 \\
0 & x
\end{array}\right)\left(\begin{array}{cc}
-1 & * \\
0 & 1
\end{array}\right)\left(\begin{array}{ll}
1 & 0 \\
1 & 1
\end{array}\right) \kappa
\end{aligned}
$$

for some $\kappa \in K_{1, v}(\mathfrak{n})$. Hence, we have

$$
\begin{aligned}
f_{\chi, s, v}\left(\left(\begin{array}{cc}
1 & 0 \\
x & 1
\end{array}\right)\right) & =f_{\chi, s, v}\left(\left(\begin{array}{cc}
-x^{-1} & 1 \\
0 & x
\end{array}\right)\left(\begin{array}{cc}
-1 & * \\
0 & 1
\end{array}\right)\left(\begin{array}{ll}
1 & 0 \\
1 & 1
\end{array}\right)\right) \\
& =\chi_{1, v}^{-1} \chi_{2, v}(x)|x|_{v}^{-(2 s+1)} f_{\chi, s, v}\left(\left(\begin{array}{ll}
1 & 0 \\
1 & 1
\end{array}\right)\right) \\
& =\chi_{1, v}^{-1} \chi_{2, v}(x)|x|_{v}^{-(2 s+1)}
\end{aligned}
$$

for $x \in F_{v}-\mathcal{O}_{v}$.

The intertwining operator $M_{v} f_{\chi, s, v}: \mathrm{GL}_{2}\left(F_{v}\right) \longrightarrow \mathbb{C}$ is defined as the integral

$$
M_{v} f_{\chi, s, v}(g)=\int_{F_{\mathfrak{p}}} f_{\chi, s, v}\left(\left(\begin{array}{cc}
0 & -1 \\
1 & 0
\end{array}\right)\left(\begin{array}{cc}
1 & n \\
0 & 1
\end{array}\right) g\right) d n
$$


for all $g \in \mathrm{GL}_{2}\left(F_{v}\right)$. This integral converges absolutely when $\operatorname{re}(s)>0$ and has meromorphic continuation to all $s$ aside from a pole at $s=t$ if $\chi_{1, v} \chi_{2, v}^{-1}(x)=|x|_{v}^{t}$ for all $x \in F_{v}^{\times}[\mathrm{B}, \S 3.7]$. Recall that for $\beta \in F^{\times}$, the integral

$$
\int_{F_{\mathfrak{p}}} f_{\chi, s, v}\left(\left(\begin{array}{cc}
0 & -1 \\
1 & 0
\end{array}\right)\left(\begin{array}{cc}
1 & n \\
0 & 1
\end{array}\right) g\right) \psi_{v}(-\beta n) d n
$$

converges absolutely when $\mathrm{re}(s)>1 / 2$ and has analytic continuation to all of $\mathbb{C}[\mathrm{B}$, loc. cit.]. In what follows, we first assume that re $(s)$ is big enough so that the above integrals converge absolutely for all $\beta \in F$, and the lemmas then follow by meromorphic continuation. We compute the following integrals for later use.

Lemma 4.1.2. For any $\left(\begin{array}{ll}a & b \\ 0 & d\end{array}\right) \in B(F)$ and for any $\beta \in F_{\mathfrak{p}}$, the integral

$$
\int_{F_{\mathfrak{p}}} f_{\chi, s, v}\left(\left(\begin{array}{cc}
0 & -1 \\
1 & 0
\end{array}\right)\left(\begin{array}{cc}
1 & n \\
0 & 1
\end{array}\right)\left(\begin{array}{ll}
a & b \\
0 & d
\end{array}\right) g\right) \psi_{v}(-\beta n) d n
$$

equals

$$
\chi_{1, v}(d) \chi_{2, v}(a)|a / d|_{v}^{\frac{1}{2}-s} \int_{F_{\mathfrak{p}}} f_{\chi, s, v}\left(\left(\begin{array}{cc}
0 & -1 \\
1 & 0
\end{array}\right)\left(\begin{array}{cc}
1 & n \\
0 & 1
\end{array}\right) g\right) \psi_{v}\left(-\beta a d^{-1} n\right) d n
$$

for all $g \in \mathrm{GL}_{2}\left(F_{\mathfrak{p}}\right)$. In particular, $M_{v} f_{\chi, s, v} \in I\left(\chi_{2, v}|\cdot|{ }_{v}^{-s}, \chi_{1, v}|\cdot| \begin{array}{l}s \\ v\end{array}\right)$.

Proof. For $\beta \in F$, we have

$$
\begin{aligned}
& \int_{F_{\mathfrak{p}}} f_{\chi, s, v}\left(\left(\begin{array}{cc}
0 & -1 \\
1 & 0
\end{array}\right)\left(\begin{array}{cc}
1 & n \\
0 & 1
\end{array}\right)\left(\begin{array}{cc}
a & b \\
0 & d
\end{array}\right)\right) \psi_{v}(-\beta n) d n \\
= & \int_{F_{\mathfrak{p}}} f_{\chi, s, v}\left(\left(\begin{array}{cc}
d & 0 \\
0 & a
\end{array}\right)\left(\begin{array}{cc}
0 & -1 \\
1 & 0
\end{array}\right)\left(\begin{array}{cc}
1 & (b+n d) a^{-1} \\
0 & 1
\end{array}\right)\right) \psi_{v}(-\beta n) d n \\
= & \chi_{1, v}(d) \chi_{2, v}(a)|d / a|^{s+\frac{1}{2}} \int_{F_{\mathfrak{p}}} f_{\chi, s, v}\left(\left(\begin{array}{cc}
0 & -1 \\
1 & 0
\end{array}\right)\left(\begin{array}{cc}
1 & (b+n d) a^{-1} \\
0 & 1
\end{array}\right)\right) \psi_{v}(-\beta n) d n \\
= & \chi_{1, v}(d) \chi_{2, v}(a)|d / a|^{s-\frac{1}{2}} \int_{F_{\mathfrak{p}}} f_{\chi, s, v}\left(\left(\begin{array}{cc}
0 & -1 \\
1 & 0
\end{array}\right)\left(\begin{array}{cc}
1 & n \\
0 & 1
\end{array}\right)\right) \psi_{v}\left(-\beta a d^{-1} n\right) d n .
\end{aligned}
$$


We now compute $f_{\chi, s, v}\left(\left(\begin{array}{cc}0 & -1 \\ 1 & 0\end{array}\right)\left(\begin{array}{ll}1 & n \\ 0 & 1\end{array}\right)\right)$, which will be used in the proof of Lemma 4.1 .3 and the proof of Lemma 4.1.4. By the Iwasawa decomposition and Lemma 2.2.3, we obtain

$$
\begin{aligned}
\left(\begin{array}{cc}
0 & -1 \\
1 & 0
\end{array}\right)\left(\begin{array}{ll}
1 & n \\
0 & 1
\end{array}\right) & =\left(\begin{array}{cc}
0 & -1 \\
1 & n
\end{array}\right) \\
& = \begin{cases}\left(\begin{array}{cc}
1 & -1 \\
0 & 1
\end{array}\right)\left(\begin{array}{ll}
1 & 0 \\
1 & 1
\end{array}\right)\left(\begin{array}{cc}
1 & n-1 \\
0 & 1
\end{array}\right) & \text { if } n \in \mathcal{O}_{\mathfrak{p}} \\
\left(\begin{array}{cc}
n^{-1} & -1 \\
0 & n
\end{array}\right)\left(\begin{array}{cc}
1 & 0 \\
n^{-1} & 1
\end{array}\right) & \text { if } n \in F_{\mathfrak{p}}-\mathcal{O}_{\mathfrak{p}},\end{cases}
\end{aligned}
$$

By Lemma 4.1.1, we see that for $n \in \mathcal{O}_{\mathfrak{p}}$, we have

$$
f_{\chi, s, v}\left(\left(\begin{array}{cc}
1 & -1 \\
0 & 1
\end{array}\right)\left(\begin{array}{ll}
1 & 0 \\
1 & 1
\end{array}\right)\left(\begin{array}{cc}
1 & n-1 \\
0 & 1
\end{array}\right)\right)= \begin{cases}0 & \text { if } e_{\mathfrak{p}, 2} \neq 0 \\
1 & \text { if } e_{\mathfrak{p}, 2}=0\end{cases}
$$

Moreover, for $n \in F_{\mathfrak{p}}-\mathcal{O}_{\mathfrak{p}}$, we have

$$
f_{\chi, s, v}\left(\left(\begin{array}{cc}
n^{-1} & -1 \\
0 & n
\end{array}\right)\left(\begin{array}{cc}
1 & 0 \\
n^{-1} & 1
\end{array}\right)\right)=\chi_{1, v}^{-1} \chi_{2, v}(n)|n|_{v}^{-(2 s+1)} f_{\chi, s, v}\left(\left(\begin{array}{cc}
1 & 0 \\
n^{-1} & 1
\end{array}\right)\right) .
$$

By Lemma 4.1.1, (4.8) implies that

$f_{\chi, s, v}\left(\left(\begin{array}{cc}n^{-1} & -1 \\ 0 & n\end{array}\right)\left(\begin{array}{cc}1 & 0 \\ n^{-1} & 1\end{array}\right)\right)= \begin{cases}\chi_{1, v}^{-1} \chi_{2, v}(n)|n|_{v}^{-(2 s+1)} & \text { if } e_{\mathfrak{p}, 1}=e_{\mathfrak{p}, 2}=0 \\ \chi_{1, v}(n) \chi_{1, v}^{-1} \chi_{2, v}(n)|n|_{v}^{-(2 s+1)} & \text { if } e_{\mathfrak{p}, 2} \neq 0 \text { and } \operatorname{val}_{v}\left(n^{-1}\right) \geq e_{\mathfrak{p}, 2} \\ 0 & \text { otherwise. }\end{cases}$

Lemma 4.1.3. Let the notation be as above.

1. If $\chi_{1, v}$ and $\chi_{2, v}$ are unramified, then

$$
M_{v} f_{\chi, s, v}\left(\left(\begin{array}{cc}
1 & 0 \\
0 & 1
\end{array}\right)\right)=\frac{1-\chi_{1, v} \chi_{2, v}^{-1}(\varpi) q^{-(2 s+1)}}{1-\chi_{1, v} \chi_{2, v}^{-1}(\varpi) q^{-2 s}}
$$

2. If $\chi_{1, v}$ is ramified and $\chi_{2, v}$ is unramified, then

$$
M_{v} f_{\chi, s, v}\left(\left(\begin{array}{ll}
1 & 0 \\
0 & 1
\end{array}\right)\right)=1
$$


3. If $\chi_{2, v}$ is ramified, then

$$
M_{v} f_{\chi, s, v}\left(\left(\begin{array}{cc}
1 & 0 \\
0 & 1
\end{array}\right)\right)=0
$$

Proof. By (4.6) and the definition of $M_{v} f_{\chi, s, v}$, we know that $M_{v} f_{\chi, s, v}\left(\left(\begin{array}{ll}1 & 0 \\ 0 & 1\end{array}\right)\right)$ equals

$$
\int_{\mathcal{O}_{\mathfrak{p}}} f_{\chi, s, v}\left(\left(\begin{array}{cc}
1 & -1 \\
0 & 1
\end{array}\right)\left(\begin{array}{cc}
1 & 0 \\
1 & 1
\end{array}\right)\left(\begin{array}{cc}
1 & n-1 \\
0 & 1
\end{array}\right)\right) d n+\int_{F_{\mathfrak{p}}-\mathcal{O}_{\mathfrak{p}}} f_{\chi, s, v}\left(\left(\begin{array}{cc}
n^{-1} & -1 \\
0 & n
\end{array}\right)\left(\begin{array}{cc}
1 & 0 \\
n^{-1} & 1
\end{array}\right)\right) d n
$$

1. Suppose that $\chi_{1, v}$ and $\chi_{2, v}$ are unramified. By (4.7), we see that

$$
\int_{\mathcal{O}_{\mathfrak{p}}} f_{\chi, s, v}\left(\left(\begin{array}{cc}
1 & -1 \\
0 & 1
\end{array}\right)\left(\begin{array}{ll}
1 & 0 \\
1 & 1
\end{array}\right)\left(\begin{array}{cc}
1 & n-1 \\
0 & 1
\end{array}\right)\right) d n=1
$$

and by (4.9), we have

$$
\int_{F_{\mathfrak{p}}-\mathcal{O}_{\mathfrak{p}}} f_{\chi, s, v}\left(\left(\begin{array}{cc}
n^{-1} & -1 \\
0 & n
\end{array}\right)\left(\begin{array}{cc}
1 & 0 \\
n^{-1} & 1
\end{array}\right)\right) d n=\int_{F_{\mathfrak{p}}-\mathcal{O}_{\mathfrak{p}}} \chi_{1, v}(n)^{-1} \chi_{2, v}(n)|n|_{\mathfrak{p}}^{-(2 s+1)} d n
$$

Hence, by (4.10), We have

$$
\begin{aligned}
\int_{F_{\mathfrak{p}}} f_{\chi, s, v}\left(\left(\begin{array}{cc}
0 & -1 \\
1 & 0
\end{array}\right)\left(\begin{array}{cc}
1 & n \\
0 & 1
\end{array}\right)\right) d n & =1+\int_{F_{\mathfrak{p}}-\mathcal{O}_{\mathfrak{p}}} \chi_{1, v}(n)^{-1} \chi_{2, v}(n)|n|_{\mathfrak{p}}^{-(2 s+1)} d n \\
& =1+\sum_{k=1}^{\infty} \int_{\varpi^{-k} \mathcal{O}_{\mathfrak{p}}^{\times}} \chi_{1, v}^{-1} \chi_{2, v}(n)|n|_{\mathfrak{p}}^{-(2 s+1)} d n \\
& =1+\sum_{k=1}^{\infty} q^{-(2 s+1) k} \int_{\varpi^{-k} \mathcal{O}_{\mathfrak{p}}^{\times}} \chi_{1, v}^{-1} \chi_{2, v}(n) d n \\
& =1+\sum_{k=1}^{\infty} q^{-(2 s+1) k} \int_{\mathcal{O}_{\mathfrak{p}}^{\times}} q^{k} \chi_{1, v}^{-1} \chi_{2, v}\left(\varpi^{-k} n\right) d n \\
& =1+\sum_{k=1}^{\infty} q^{-2 s k} \chi_{1, v} \chi_{2, v}^{-1}\left(\varpi^{k}\right) \times \frac{q-1}{q} \\
& =\frac{1-\chi_{1, v} \chi_{2, v}^{-1}(\varpi) q^{-2 s-1}}{1-\chi_{1, v} \chi_{2, v}^{-1}(\varpi) q^{-2 s}} .
\end{aligned}
$$

2. Suppose that $\chi_{1}$ is ramified and $\chi_{2}$ is unramified. By (4.7), we know that

$$
\int_{\mathcal{O}_{\mathfrak{p}}} f_{\chi, s, v}\left(\left(\begin{array}{cc}
1 & -1 \\
0 & 1
\end{array}\right)\left(\begin{array}{ll}
1 & 0 \\
1 & 1
\end{array}\right)\left(\begin{array}{cc}
1 & n-1 \\
0 & 1
\end{array}\right)\right) d n=1
$$


and by (4.9), we have

$$
\int_{F_{\mathfrak{p}}-\mathcal{O}_{\mathfrak{p}}} f_{\chi, s, v}\left(\left(\begin{array}{cc}
n^{-1} & -1 \\
0 & n
\end{array}\right)\left(\begin{array}{cc}
1 & 0 \\
n^{-1} & 1
\end{array}\right)\right) d n=0
$$

Thus the assertion follows from (4.10).

3. If $\chi_{1, v}$ is unramified and $\chi_{2, v}$ is ramified, then by (4.7), we know that

$$
\int_{\mathcal{O}_{\mathfrak{p}}} f_{\chi, s, v}\left(\left(\begin{array}{cc}
1 & -1 \\
0 & 1
\end{array}\right)\left(\begin{array}{ll}
1 & 0 \\
1 & 1
\end{array}\right)\left(\begin{array}{cc}
1 & n-1 \\
0 & 1
\end{array}\right)\right) d n=0
$$

and by (4.9), we have

$\int_{F_{\mathfrak{p}}-\mathcal{O}_{\mathfrak{p}}} f_{\chi, s, v}\left(\left(\begin{array}{cc}n^{-1} & -1 \\ 0 & n\end{array}\right)\left(\begin{array}{cc}1 & 0 \\ n^{-1} & 1\end{array}\right)\right) d n=\sum_{k=e_{\mathfrak{p}, 2}}^{\infty} \chi_{1, v}\left(\varpi^{e_{\mathfrak{p}, 2}}\right) \int_{\varpi^{-k} \mathcal{O}_{\mathfrak{p}}^{\times}} \chi_{1, v}^{-1} \chi_{2, v}(n)|n|_{\mathfrak{p}}^{-(2 s+1)} d n$

It follows from (4.10) that we have

$$
\begin{aligned}
\int_{F_{\mathfrak{p}}} f_{\chi, s, v}\left(\left(\begin{array}{cc}
0 & -1 \\
1 & 0
\end{array}\right)\left(\begin{array}{cc}
1 & n \\
0 & 1
\end{array}\right)\right) d n & =\sum_{k=e_{\mathfrak{p}, 2}}^{\infty} \chi_{1, v}\left(\varpi^{e_{\mathfrak{p}, 2}}\right) \int_{\varpi^{-k} \mathcal{O}_{\mathfrak{p}}^{\times}} \chi_{1, v}^{-1} \chi_{2, v}(n)|n|_{\mathfrak{p}}^{-(2 s+1)} d n \\
& =\sum_{k=e_{\mathfrak{p}, 2}}^{\infty} \chi_{1, v}\left(\varpi^{e_{\mathfrak{p}, 2}}\right) \chi_{1, v} \chi_{2, v}^{-1}\left(\varpi^{k}\right)|\varpi|_{\mathfrak{p}}^{2 s k} \int_{\mathcal{O}_{\mathfrak{p}}^{\times}} \chi_{2, v}(x) d x \\
& =0 .
\end{aligned}
$$

The last integral is 0 since the character $\chi_{2, v}$ is not trivial on $\mathcal{O}_{\mathfrak{p}}^{\times}$.

If $\chi_{1, v}$ and $\chi_{2, v}$ are ramified, then similarly by (4.7) and (4.9), we have

$$
\begin{aligned}
\int_{F_{\mathfrak{p}}} f_{\chi, s, v}\left(\left(\begin{array}{cc}
0 & -1 \\
1 & 0
\end{array}\right)\left(\begin{array}{cc}
1 & n \\
0 & 1
\end{array}\right)\right) d n & =\int_{\varpi^{-e_{\mathfrak{p}, 2} \mathcal{O}_{\mathfrak{p}}^{\times}}} \chi_{1, v}(x) \chi_{1, v}^{-1}(x) \chi_{2, v}(x)|x|_{\mathfrak{p}}^{-(2 s+1)} d x \\
& =|\varpi|_{\mathfrak{p}}^{2 s e_{\mathfrak{p}, 2}} \int_{\mathcal{O}_{\mathfrak{p}}^{\times}} \chi_{2, v}(x) d x \\
& =0 .
\end{aligned}
$$

The last equality is again obtained by the assumption that $\chi_{2, v}$ is ramified. 
Recall that for each finite place $v$ of $F$, we fix an additive character $\psi_{v}$ on $F_{\mathfrak{p}}$ which is defined as

$$
\psi_{v}(x)=e^{-2 \pi i\left[\operatorname{Tr}_{F_{\mathfrak{p}} / \mathbb{Q}_{p}}(x)\right]_{p}} .
$$

For a number $\beta \in F$, we set $\gamma_{v}(\beta):=\int_{\mathcal{O}_{\mathfrak{p}}} \psi_{v}(-\beta n) d n$. It is 1 if $\operatorname{Tr}_{F_{\mathfrak{p}} / \mathbb{Q}_{p}}(\beta) \in \mathbb{Z}_{p}$; otherwise, it is 0 (Lemma 2.3.1(3)). Also, recall that for a finite place $v$ of $F$ with $v \mid \mathfrak{n}_{2}$, the local epsilon factor $\varepsilon_{v}\left(s, \chi_{2, v}^{-1}, \psi_{v}\right)$ is defined as the following integral [Tate]

$$
\varepsilon_{v}\left(s, \chi_{2, v}^{-1}, \psi_{v}\right):=\int_{F_{\mathfrak{p}}^{\times}} \chi_{2, v}(n)|n|^{-s} \psi_{v}(n) d n .
$$

This integral converges absolutely when $\operatorname{re}(s)>3 / 2$ and has analytic continuation to all $s$ without having any zeros and poles [B, Proposition 3.1.9]. Since we assume that $\mathfrak{n}_{2}$ and $\mathfrak{D}$ are coprime, by Lemma 2.4.1 we have

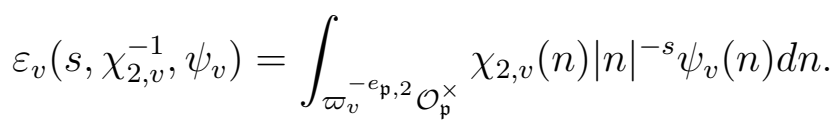

Lemma 4.1.4. Let $\beta \in F$.

1. Assume that the conductor of $\psi_{v}$ is $\varpi^{-r}$ for some $r \in \mathbb{Z}_{\geq 0}$, and assume that $\chi_{1, v}$ and $\chi_{2, v}$ are unramified. Then

$$
\begin{aligned}
& \int_{F_{\mathfrak{p}}} f_{\chi, s, v}\left(\left(\begin{array}{cc}
0 & -1 \\
1 & 0
\end{array}\right)\left(\begin{array}{cc}
1 & n \\
0 & 1
\end{array}\right)\right) \psi_{v}(-\beta n) d n \\
= & \left(1-\chi_{1, v} \chi_{2, v}^{-1}\left(\varpi_{v}\right) q^{-(2 s+1)}\right) \sum_{n=0}^{r+\operatorname{val}_{v}(\beta)} \chi_{1, v} \chi_{2, v}^{-1}\left(\varpi_{v}^{n}\right) q^{-2 s n}
\end{aligned}
$$

if $\operatorname{val}_{v}(\beta)+r \geq 0$. Otherwise, the integral is 0 .

2. If $\chi_{1, v}$ is ramified and $\chi_{2, v}$ is unramified, then

$$
\int_{F_{\mathfrak{p}}} f_{\chi, s, v}\left(\left(\begin{array}{cc}
0 & -1 \\
1 & 0
\end{array}\right)\left(\begin{array}{cc}
1 & n \\
0 & 1
\end{array}\right)\right) \psi_{v}(-\beta n) d n=\gamma_{v}(\beta)
$$


3. If $\chi_{2, v}$ is ramified and $\chi_{1, v}$ is unramified, then

$$
\int_{F_{\mathfrak{p}}} f_{\chi, s, v}\left(\left(\begin{array}{cc}
0 & -1 \\
1 & 0
\end{array}\right)\left(\begin{array}{cc}
1 & n \\
0 & 1
\end{array}\right)\right) \psi_{v}(-\beta n) d n=\chi_{1, v} \chi_{2, v}^{-1}(-\beta)|\beta|_{v}^{2 s} \varepsilon_{v}\left(2 s+1, \chi_{2, v}^{-1}, \psi_{v}\right)
$$

if $\operatorname{val}(\beta) \geq 0$; otherwise, the integral is 0 .

4. If $\chi_{1, v}$ and $\chi_{2, v}$ are ramified, then

$$
\int_{F_{\mathfrak{p}}} f_{\chi, s, v}\left(\left(\begin{array}{cc}
0 & -1 \\
1 & 0
\end{array}\right)\left(\begin{array}{cc}
1 & n \\
0 & 1
\end{array}\right)\right) \psi_{v}(-\beta n) d n=\chi_{2, v}^{-1}(-\beta) \varepsilon_{v}\left(2 s+1, \chi_{2, v}^{-1}, \psi_{v}\right)
$$

if $\operatorname{val}(\beta)=0$; otherwise, the integral is 0 .

ProOF. Similarly to the proof of Lemma 4.1.3, we have to compute two integrals for each case.

One is

$$
\int_{\mathcal{O}_{\mathfrak{p}}} f_{\chi, s, v}\left(\left(\begin{array}{cc}
1 & -1 \\
0 & 1
\end{array}\right)\left(\begin{array}{ll}
1 & 0 \\
1 & 1
\end{array}\right)\left(\begin{array}{cc}
1 & n-1 \\
0 & 1
\end{array}\right)\right) \psi_{v}(-\beta n) d n
$$

The other is

$$
\int_{F_{\mathfrak{p}}-\mathcal{O}_{\mathfrak{p}}} f_{\chi, s, v}\left(\left(\begin{array}{cc}
n^{-1} & -1 \\
0 & n
\end{array}\right)\left(\begin{array}{cc}
1 & 0 \\
n^{-1} & 1
\end{array}\right)\right) \psi_{v}(-\beta n) d n
$$

1. Assume that both $\chi_{1, v}$ and $\chi_{2, v}$ are unramified. By (4.7), we know that (4.11) is $\gamma_{v}(\beta)$ which is 1 if $\operatorname{val}_{v}(\beta) \geq-r$ and is 0 otherwise (Lemma 2.3.1(3)). By (4.9), we see that (4.12) equals

$$
\begin{aligned}
& \sum_{k=1}^{\infty} \int_{\varpi^{-k} \mathcal{O}_{\mathfrak{p}}^{\times}} \chi_{1, v}^{-1} \chi_{2, v}(n)|n|^{-(2 s+1)} \psi_{v}(-\beta n) d n \\
= & \sum_{k=1}^{\infty} \chi_{1, v} \chi_{2, v}^{-1}\left(\varpi^{k}\right)\left|\varpi^{k}\right|^{2 s} \int_{\mathcal{O}_{\mathfrak{p}}^{\times}} \psi_{v}\left(-\beta \varpi^{-k} n\right) d n \\
= & \sum_{k=1}^{\infty} \chi_{1, v} \chi_{2, v}^{-1}\left(\varpi^{k}\right)\left|\varpi^{k}\right|^{2 s}\left(\int_{\mathcal{O}_{\mathfrak{p}}} \psi_{v}\left(-\beta \varpi^{-k} n\right) d n-\int_{\varpi \mathcal{O}_{\mathfrak{p}}} \psi_{v}\left(-\beta \varpi^{-k} n\right) d n\right) .
\end{aligned}
$$

Again by Lemma 2.3.1(3), we know that the integral $\int_{\mathcal{O}_{\mathfrak{p}}} \psi_{v}\left(-\beta \varpi^{-k} n\right) d n$ is 1 if $\operatorname{val}_{v}(\beta)-k \geq-r$; otherwise, it is 0. Similarly, by Lemma 2.3.1(4), the integral $\int_{\varpi \mathcal{O}_{\mathfrak{p}}} \psi_{v}\left(-\beta \varpi^{-k} n\right) d n$ is $q^{-1}$ if $\operatorname{val}_{v}(\beta)-k+1 \geq-r$; otherwise, it is 0 . Note that since $k$ is a positive integer, the above two 
integrals are 0 if $\operatorname{val}_{v}(\beta)+r<0$. In such cases, we have seen that (4.12) is 0 . Moreover, if $\operatorname{val}_{v}(\beta)+r=0$, then the first integral is 0 and the second integral is $q^{-1}$. In such a case, we have shown that $(4.12)$ is

$$
-q^{-1} \chi_{1, v} \chi_{2, v}^{-1}(\varpi)|\varpi|^{2 s}=\chi_{1, v} \chi_{2, v}^{-1}(\varpi) q^{-(2 s+1)}
$$

Now we assume that $\operatorname{val}_{v}(\beta)+r>0$. Then the first integral is 1 if $k \leq \operatorname{val}_{v}(\beta)+r$; otherwise, it is 0 , and the second integral is $q^{-1}$ if $k \leq \operatorname{val}_{v}(\beta)+r+1$; otherwise, it is 0 . Thus, we see that (4.12) equals

$$
\begin{aligned}
& \sum_{k=1}^{\operatorname{val}_{v}(\beta)+r} \chi_{1, v} \chi_{2, v}^{-1}\left(\varpi^{k}\right)\left|\varpi^{k}\right|^{2 s}-q^{-1} \sum_{k=1}^{\operatorname{val}_{v}(\beta)+r+1} \chi_{1, v} \chi_{2, v}^{-1}\left(\varpi^{k}\right)\left|\varpi^{k}\right|^{2 s} \\
= & \frac{q-1}{q} \sum_{k=1}^{\operatorname{val}_{v}(\beta)+r}\left(\chi_{1, v} \chi_{2, v}^{-1}\left(\varpi^{k}\right) q^{-2 s k}\right)-\frac{1}{q} \chi_{1, v} \chi_{2, v}^{-1}\left(\varpi^{r+\operatorname{val}_{v}(\beta)+1}\right) q^{-2 s\left(r+\operatorname{val}_{v}(\beta)+1\right)} .
\end{aligned}
$$

To sum up, we have shown that if $\operatorname{val}_{v}(\beta)>-r$, then we have

$$
\begin{aligned}
& \int_{F_{\mathfrak{p}}} f_{\chi, s, v}\left(\left(\begin{array}{cc}
0 & -1 \\
1 & 0
\end{array}\right)\left(\begin{array}{cc}
1 & n \\
0 & 1
\end{array}\right)\right) \psi_{v}(-\beta n) d n \\
= & 1+\left[\frac{q-1}{q} \sum_{k=1}^{r+\operatorname{val}_{v}(\beta)}\left(\chi_{1, v} \chi_{2, v}^{-1}\left(\varpi^{k}\right) q^{-2 s k}\right)-\frac{1}{q} \chi_{1, v} \chi_{2, v}^{-1}\left(\varpi^{r+\operatorname{val}_{v}(\beta)+1}\right) q^{-2 s\left(r+\operatorname{val}_{v}(\beta)+1\right)}\right] \\
= & { }^{(*)}\left(1-\chi_{1, v} \chi_{2, v}^{-1}(\varpi) q^{-(2 s+1)}\right) \times \frac{1-\left(\chi_{1, v} \chi_{2, v}^{-1}(\varpi) q^{-2 s}\right)^{\operatorname{val}_{v}(\beta)+1}}{1-\chi_{1, v} \chi_{2, v}^{-1}(\varpi) q^{-2 s}} \\
= & \left(1-\chi_{1, v} \chi_{2, v}^{-1}(\varpi) q^{-(2 s+1)}\right) \times \sum_{n=0}^{r+\operatorname{val}_{v}(\beta)}\left(\chi_{1, v} \chi_{2, v}^{-1}(\varpi) q^{-2 s n}\right) .
\end{aligned}
$$

Here the equality $(*)$ was obtained by direct computation. Moreover, when $\operatorname{val}_{v}(\beta)=-r$, we have shown that

$$
\int_{F_{\mathfrak{p}}} f_{\chi, s, v}\left(\left(\begin{array}{cc}
0 & -1 \\
1 & 0
\end{array}\right)\left(\begin{array}{cc}
1 & n \\
0 & 1
\end{array}\right)\right) \psi_{v}(-\beta n) d n=1-\chi_{1, v} \chi_{2, v}^{-1}(\varpi) q^{-(2 s+1)}
$$

and if $\operatorname{val}_{v}(\beta)<-r$, then the integral is 0 . 
2. Assume that $\chi_{1, v}$ is ramified and $\chi_{2, v}$ us unramified. It follows from (4.7) that $(4.11)$ is $\gamma_{v}(\beta)$. In addition, it follows from (4.9) that (4.12) is 0 . This completes the proof of the second assertion.

3. Assume that $\chi_{1, v}$ is unramified and $\chi_{2, v}$ is ramified. It follows from (4.7) that (4.11) is 0 , and it follows from (4.9) that (4.12) equals

$$
\begin{aligned}
& \sum_{k=0}^{\infty} \int_{\varpi^{-\left(e_{\mathfrak{p}, 2}+k\right)}} \chi_{1, v}^{-1} \chi_{2, v}(n)|n|_{v}^{-(2 s+1)} \chi_{1, v}\left(\varpi^{-e_{\mathfrak{p}, 2}}\right) \psi_{v}(-\beta n) d n \\
= & \sum_{k=0}^{\infty} \chi_{1, v}\left(-\beta \varpi^{-e_{\mathfrak{p}, 2}}\right) \chi_{2, v}^{-1}(-\beta)|\beta|_{v}^{2 s} \int_{\varpi^{-\left(e_{\mathfrak{p}, 2}+k\right)+\operatorname{val}(\beta)} \mathcal{O}_{\mathfrak{p}}^{\times}} \chi_{1, v}^{-1} \chi_{2, v}(x)|x|_{v}^{-(2 s+1)} \psi_{v}(x) d x .
\end{aligned}
$$

By Lemma 2.4.1, we know that the above integral is 0 if $k \neq \operatorname{val}_{v}(\beta)$, which is always true if $\operatorname{val}_{v}(\beta)<0$ since $k$ is a non-negative integer. We now assume that $\operatorname{val}_{v}(\beta) \geq 0$. Then (4.12) equals

$$
\begin{aligned}
& \chi_{1, v}\left(-\beta \varpi^{-e_{\mathfrak{p}, 2}}\right) \chi_{2, v}^{-1}(-\beta)|\beta|_{v}^{2 s} \int_{\varpi^{-e_{\mathfrak{p}, 2} \mathcal{O}_{\mathfrak{p}}^{\times}}} \chi_{1, v}^{-1} \chi_{2, v}(x)|x|_{v}^{-(2 s+1)} \psi_{v}(x) d x \\
& =\chi_{1, v} \chi_{2, v}^{-1}(-\beta)|\beta|_{v}^{2 s} \int_{\varpi^{-e_{\mathfrak{p}, 2} \mathcal{O}_{\mathfrak{p}}^{\times}}} \chi_{2, v}(x)|x|_{v}^{-(2 s+1)} \psi_{v}(x) d x \\
& =\chi_{1, v} \chi_{2, v}^{-1}(-\beta)|\beta|_{v}^{2 s} \varepsilon_{v}\left(2 s+1, \chi_{2, v}^{-1}, \psi_{v}\right) .
\end{aligned}
$$

if $\operatorname{val}(\beta) \geq 0$; otherwise, the integral is 0 . Note that the second equality holds since $\chi-1, v$ is unramified.

4. Assume that both $\chi_{1, v}$ and $\chi_{2, v}$ are ramified. It follows from (4.7) that (4.11) is 0 , and it follows from (4.9) that (4.12) equals

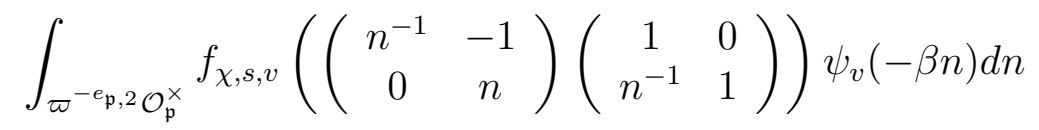

$$
\begin{aligned}
& =\int_{\varpi^{-e_{\mathfrak{p}, 2} \mathcal{O}_{\mathfrak{p}}^{\times}}} \chi_{1}^{-1} \chi_{2, v}(n)|n|_{v}^{-(2 s+1)} \chi_{1, v}(n) \psi_{v}(-\beta n) d n \\
& =\chi_{2, v}^{-1}(-\beta)|\beta|_{v} \int_{\varpi^{-e_{\mathfrak{p}, 2}+\operatorname{val}(\beta)} \mathcal{O}_{\mathfrak{p}}^{\times}} \chi_{2, v}(x)|x|_{v}^{-(2 s+1)} \psi_{v}(x) d x \\
& =\chi_{2, v}^{-1}(-\beta)|\beta|^{2 s} \varepsilon_{v}\left(2 s+1, \chi_{2, v}^{-1}, \psi_{v}\right) .
\end{aligned}
$$


By Lemma 2.4.1, the last equality holds if $\operatorname{val}(\beta)=0$; otherwise, the integral is 0 . This completes the proof.

To state Lemma 4.1.5, we recall some notations defined in Chapter 2. For a positive integer $n$, we put $U_{\mathfrak{p}}^{(n)}:=\mathcal{O}_{\mathfrak{p}}^{\times} / 1+\varpi^{n} \mathcal{O}_{\mathfrak{p}}$. For $i=1,2$, we set

$$
\tau\left(\chi_{i, v}\right):=\sum_{x \in U_{\mathfrak{p}}^{\left(e_{\mathfrak{p}, i}\right)}} \chi_{i, v}(x) \psi_{v}\left(\varpi^{-e_{\mathfrak{p}, i}} x\right)
$$

Here we view $\chi_{i, v}$ as a character on $\mathcal{O}_{\mathfrak{p}}^{\times} / 1+\varpi^{e_{\mathfrak{p}, i}} \mathcal{O}_{\mathfrak{p}} \simeq\left(\mathcal{O}_{\mathfrak{p}} / \varpi^{e_{\mathfrak{p}, i}}\right)^{\times}$. One can extend it to a character on $\mathcal{O}_{\mathfrak{p}} / \varpi^{e_{\mathfrak{p}, i}}$ by setting $\chi_{i, v}(x)=0$ if $x \equiv 0 \bmod \varpi$. Then we have

$$
\tau\left(\chi_{i, v}\right)=\sum_{x \in \mathcal{O}_{\mathfrak{p}} / \varpi^{e_{\mathfrak{p}, i}}} \chi_{i, v}(x) \psi_{v}\left(\varpi^{-e_{\mathfrak{p}, i}} x\right)
$$

Moreover, we set

$$
J_{a}\left(\chi_{1, v}, \chi_{2, v}, \varpi^{k}\right):=\sum_{x \in\left(\mathcal{O}_{\mathfrak{p}} / \varpi^{k} \mathcal{O}_{\mathfrak{p}}\right) \times} \chi_{1, v}(x) \chi_{2, v}(a-x)=\sum_{x \in \mathcal{O}_{\mathfrak{p}} / \varpi^{k} \mathcal{O}_{\mathfrak{p}}} \chi_{1, v}(x) \chi_{2, v}(a-x)
$$

for $a \in \mathcal{O}_{\mathfrak{p}}$ and $k \geq \max \left\{e_{\mathfrak{p}, 1}, e_{\mathfrak{p}, 2}\right\}$. It is easy to see that $J_{a}\left(\chi_{1, v}, \chi_{2, v}, \varpi^{k}\right)=J_{a}\left(\chi_{2, v}, \chi_{1, v}, \varpi^{k}\right)$, and $J_{a}\left(\chi_{1, v} \theta, \chi_{2, v}, \varpi^{k}\right)=J_{a}\left(\chi_{1, v}, \chi_{2, v}, \varpi^{k}\right)$ if $\theta$ is unramified.

We now compute $f_{\chi, s, v}\left(\left(\begin{array}{cc}0 & -1 \\ 1 & 0\end{array}\right)\left(\begin{array}{ll}1 & n \\ 0 & 1\end{array}\right)\left(\begin{array}{cc}1 & 0 \\ \varpi^{i} & 1\end{array}\right)\right)$ for $i \in \mathbb{Z}_{\geq 0}$ and $n \in F_{v}$, which will be used in the proof of Lemma 4.1.5. First of all, we have

$$
\left(\begin{array}{cc}
0 & -1 \\
1 & 0
\end{array}\right)\left(\begin{array}{cc}
1 & n \\
0 & 1
\end{array}\right)\left(\begin{array}{cc}
1 & 0 \\
\varpi^{i} & 1
\end{array}\right)=\left(\begin{array}{cc}
-\varpi^{i} & -1 \\
1+\varpi^{i} n & n
\end{array}\right)
$$

When $i \neq 0$, by Lemma 2.2.3 for $n \in \mathcal{O}_{v}$ and by Lemma 2.2.2 for $n \in F_{v}-\mathcal{O}_{v}$, we have

$$
\left(\begin{array}{cc}
-\varpi^{i} & -1 \\
1+\varpi^{i} n & n
\end{array}\right)=\left\{\begin{array}{cc}
\left(\begin{array}{cc}
\left.1+n \varpi^{i}\right)^{-1} & * \\
0 & 1+n \varpi^{i}
\end{array}\right)\left(\begin{array}{cc}
1 & 0 \\
1 & 1
\end{array}\right) \kappa & \text { if } n \in \mathcal{O}_{v} \\
\left(\begin{array}{cc}
n^{-1} & * \\
0 & n
\end{array}\right)\left(\begin{array}{cc}
1 & -0 \\
\left(1+n \varpi^{i}\right) n^{-1} & 1
\end{array}\right) & \text { if } n \in F_{v}-\mathcal{O}_{v}
\end{array}\right.
$$


for some $\kappa \in K_{1, v}(\mathfrak{n})$. Thus, for $n \in \mathcal{O}_{v}$, by Lemma 4.1.1, we have

$$
f_{\chi, s, v}\left(\left(\begin{array}{cc}
0 & -1 \\
1 & 0
\end{array}\right)\left(\begin{array}{cc}
1 & n \\
0 & 1
\end{array}\right)\left(\begin{array}{cc}
1 & 0 \\
\varpi^{i} & 1
\end{array}\right)\right)= \begin{cases}0 & \text { if } e_{\mathfrak{p}, 2} \neq 0 \\
\chi_{1, v}^{-1}\left(1+n \varpi^{i}\right) & \text { if } e_{\mathfrak{p}, 2}=0\end{cases}
$$

Moreover, for $n \in F_{v}-\mathcal{O}_{v}$, we have

$$
f_{\chi, s, v}\left(\left(\begin{array}{cc}
0 & -1 \\
1 & 0
\end{array}\right)\left(\begin{array}{cc}
1 & n \\
0 & 1
\end{array}\right)\left(\begin{array}{cc}
1 & 0 \\
\varpi^{i} & 1
\end{array}\right)\right)=\chi_{1, v}^{-1} \chi_{2, v}(n)|n|^{-(2 s+1)} f_{\chi, s, v}\left(\left(\begin{array}{cc}
1 & 0 \\
\left(1+n \varpi^{i}\right) n^{-1} & 1
\end{array}\right)\right) .
$$

In the proof of Lemma 4.1.5, we will always evaluate $f_{\chi, s, v}\left(\left(\begin{array}{c}1 \\ \left(1+n \varpi^{i}\right) n^{-1}\end{array} \begin{array}{l}0 \\ 1\end{array}\right)\right)$ by Lemma 4.1.1.

Lemma 4.1.5. Let $i<e_{\mathfrak{p}, 1}+e_{\mathfrak{p}, 2}$ be a non-negative integer, and let $M_{v, s, i}:=M_{v} f_{\chi, s, v}\left(\left(\begin{array}{cc}1 & 0 \\ \varpi^{i} & 1\end{array}\right)\right)$.

1. Suppose one of the following conditions holds

(a) $\chi_{1, v}$ is unramified, $\chi_{2, v}$ is ramified, and $0<i<e_{\mathfrak{p}, 2}$.

(b) $\chi_{1, v}$ is ramified, $\chi_{2, v}$ is unramified, and $0 \leq i<e_{\mathfrak{p}, 1}$.

(c) $\chi_{1, v}$ and $\chi_{2, v}$ are ramified, $0 \leq i<e_{\mathfrak{p}, 1}+e_{\mathfrak{p}, 2}$, and $i \neq e_{\mathfrak{p}, 1}$.

Then $M_{v, s, i}=0$.

2. If $\chi_{1, v}$ is unramified and $\chi_{2, v}$ is ramified, then

$$
M_{v, s, 0}=\chi_{1, v}^{-1}\left(\varpi^{e_{\mathfrak{p}, 2}}\right) \chi_{2, v}(-1) q_{v}^{-e_{\mathfrak{p}, 2}}
$$

3. If both $\chi_{1, v}$ and $\chi_{2, v}$ are ramified, let $\varpi^{t}$ be the conductor of $\chi_{1, v}^{-1} \chi_{2, v}$.

(3.1) If $e_{\mathfrak{p}, 1}>e_{\mathfrak{p}, 2}$, then

$$
M_{v, s, e_{\mathfrak{p}, 1}}=\chi_{2, v}\left(-\varpi^{-e_{\mathfrak{p}, 2}}\right)\left|\varpi^{e_{\mathfrak{p}, 2}}\right|^{2 s+1} \sum_{x \in U_{\mathfrak{p}}^{\left(\boldsymbol{e}_{\mathfrak{p}, 2}\right)}} \chi_{2, v}(x) \chi_{1, v}^{-1}\left(1-\varpi^{e_{\mathfrak{p}, 1}-e_{\mathfrak{p}, 2}} x\right) .
$$




$$
\text { If } e_{\mathfrak{p}, 1}<e_{\mathfrak{p}, 2} \text {, then }
$$

$$
M_{v, s, e_{\mathfrak{p}, 1}}=\chi_{1, v}^{-1}\left(\varpi^{e_{\mathfrak{p}, 2}-e_{\mathfrak{p}, 1}}\right) \chi_{2, v}\left(-\varpi^{-e_{\mathfrak{p}, 1}}\right)|\varpi|^{2 s e_{\mathfrak{p}, 1}+e_{\mathfrak{p}, 2}} \sum_{x \in U_{\mathfrak{p}}^{\left(e_{\mathfrak{p}, 1}\right)}} \chi_{1, v}^{-1}(x) \chi_{2, v}\left(1-\varpi^{e_{\mathfrak{p}, 2}-e_{\mathfrak{p}, 1}} x\right) .
$$

(3.3) If $e_{\mathfrak{p}, 1}=e_{\mathfrak{p}, 2}$ and if $t=e_{\mathfrak{p}, 1}$, then

$$
M_{v, s, e_{\mathfrak{p}, 1}}=\chi_{2, v}\left(-\varpi^{-e_{\mathfrak{p}, 1}}\right)|\varpi|^{e_{\mathfrak{p}, 1}(2 s+1)} J_{1}\left(\chi_{2, v}, \chi_{1, v}^{-1}, \varpi^{e_{\mathfrak{p}, 1}}\right) .
$$

(3.4) If $e_{\mathfrak{p}, 1}=e_{\mathfrak{p}, 2}$ and if $1 \leq t<e_{\mathfrak{p}, 1}$, then

$$
M_{v, s, e_{\mathfrak{p}, 1}}=\chi_{1, v}^{-1}\left(\varpi^{t-e_{\mathfrak{p}, 1}}\right) \chi_{2, v}\left(-\varpi^{t-2 e_{\mathfrak{p}, 1}}\right)|\varpi|^{2 s\left(2 e_{\mathfrak{p}, 1}-t\right)+e_{\mathfrak{p}, 1}} J_{\varpi_{\mathfrak{p}, 1}-t}\left(\chi_{2, v}, \chi_{1, v}^{-1}, \varpi^{e_{\mathfrak{p}, 1}}\right) .
$$

(3.5) If $e_{\mathfrak{p}, 1}=e_{\mathfrak{p}, 2}$ and if $t=0$, then

$$
\begin{aligned}
M_{v, s, e_{\mathfrak{p}, 1}=} & \chi_{1, v}^{-1}\left(\varpi^{e_{\mathfrak{p}, 1}}\right) \chi_{2, v}(-1)|\varpi|^{2 s\left(2 e_{\mathfrak{p}, 1}-1\right)+e_{\mathfrak{p}, 1}} J_{\varpi^{e_{p}, 1}-1}\left(\chi_{2, v}, \chi_{1, v}^{-1}, \varpi^{e_{\mathfrak{p}, 1}}\right)+ \\
& \sum_{k=2 e_{\mathfrak{p}, 1}}^{\infty} \chi_{1, v}^{-1}\left(\varpi^{e_{\mathfrak{p}, 1}}\right) \chi_{2, v}(-1)|\varpi|^{2 s k+e_{\mathfrak{p}, 1}} J_{0}\left(\chi_{1, v}^{-1}, \chi_{2, v}, \varpi^{e_{\mathfrak{p}, 1}}\right) .
\end{aligned}
$$

\section{Proof.}

1. We have shown in Lemma 4.1.2 that $M_{v} f_{\chi, s, v} \in I\left(\chi_{2, v}|\cdot|^{-1}, \chi_{1 . v}|\cdot|\right)$. Moreover, it satisfies

$$
M_{v} f_{\chi, s, v}\left(g\left(\begin{array}{ll}
a & b \\
c & d
\end{array}\right)\right)=M_{v} f_{\chi, s, v}(g) \chi_{1, v} \chi_{2, v}(d)
$$

for $g \in \mathrm{GL}_{2}\left(F_{v}\right)$ by the definition of $f_{\chi, s, v}$. Then the assertion follows from the same argument as in Lemma 4.1.1.

2. Assume that $\chi_{1, v}$ is unramified and $\chi_{2, v}$ is ramified. We decompose $M_{v, s, 0}$ into two integrals. The first one is an integral over $\mathcal{O}_{p}$ :

$$
\int_{\mathcal{O}_{p}} f_{\chi, s, v}\left(\left(\begin{array}{cc}
0 & -1 \\
1 & 0
\end{array}\right)\left(\begin{array}{cc}
1 & n \\
0 & 1
\end{array}\right)\left(\begin{array}{ll}
1 & 0 \\
1 & 1
\end{array}\right)\right) d n
$$


By (4.13), it equals

$$
\int_{\mathcal{O}_{\mathfrak{p}}} f_{\chi, s, v}\left(\left(\begin{array}{cc}
-1 & -1 \\
1+n & n
\end{array}\right)\right) d n=\int_{\mathcal{O}_{\mathfrak{p}}} f_{\chi, s, v}\left(\left(\begin{array}{cc}
-1 & -1 \\
n & n-1
\end{array}\right)\right) d n
$$

Since $\chi_{1, v}$ is unramified and $\chi_{2, v}$ is ramified, by Lemma 4.1.1(2), we know that $f_{\chi, s, v}\left(\left(\begin{array}{cc}-1 & -1 \\ n & n-1\end{array}\right)\right)$ is 0 if and only if $\operatorname{val}_{v}(n)<e_{\mathfrak{p}, 2}$, which follows that the above integral equals

$$
\begin{aligned}
\sum_{k=e_{\mathfrak{p}, 2}}^{\infty} \int_{\varpi^{k} \mathcal{O}_{\mathfrak{p}}^{\times}} f_{\chi, s, v}\left(\left(\begin{array}{cc}
-1 & -1 \\
n & n-1
\end{array}\right)\right) d n & =\sum_{k=e_{\mathfrak{p}, 2}}^{\infty} \int_{\varpi^{k} \mathcal{O}_{\mathfrak{p}}^{\times}} f_{\chi, s, v}\left(\left(\begin{array}{cc}
\frac{1}{n-1} & * \\
0 & n-1
\end{array}\right)\left(\begin{array}{cc}
1 & 0 \\
\frac{n}{n-1} & 1
\end{array}\right)\right) d n \\
& =\sum_{k=e_{\mathfrak{p}, 2}}^{\infty} \int_{\varpi^{k} \mathcal{O}_{\mathfrak{p}}^{\times}} \chi_{1, v}^{-1} \chi_{2, v}(n-1) \chi_{1, v}^{-1}\left(\varpi^{e_{\mathfrak{p}, 2}}\right) d n \\
& =\chi_{1, v}^{-1}\left(\varpi^{e_{\mathfrak{p}, 2}}\right) \chi_{2, v}(-1) \sum_{k=e_{\mathfrak{p}, 2}}^{\infty} \int_{\varpi^{k} \mathcal{O}_{\mathfrak{p}}^{\times}} 1 d n \\
& =\chi_{1, v}^{-1}\left(\varpi^{e_{\mathfrak{p}, 2}}\right) \chi_{2, v}(-1) \sum_{k=e_{\mathfrak{p}, 2}}^{\infty} q_{v}^{-k} \times \frac{q_{v}-1}{q_{v}} \\
& =\chi_{1, v}^{-1}\left(\varpi^{e_{\mathfrak{p}, 2}}\right) \chi_{2, v}(-1) \frac{q_{v}^{-e_{\mathfrak{p}, 2}}}{1-q_{v}^{-1}} \times \frac{q_{v}-1}{q_{v}} \\
& =\chi_{1, v}^{-1}\left(\varpi^{e_{\mathfrak{p}, 2}}\right) \chi_{2, v}(-1) q_{v}^{-e_{\mathfrak{p}, 2} .}
\end{aligned}
$$

Note that the first equality is obtained by Lemma 2.2.3, and the second equality is obtained by the definition of $f_{\chi, s, v}$. The second integral is over $F_{\mathfrak{p}}-\mathcal{O}_{\mathfrak{p}}$ :

$$
\int_{F_{v}-\mathcal{O}_{v}} f_{\chi, s, v}\left(\left(\begin{array}{cc}
0 & -1 \\
1 & 0
\end{array}\right)\left(\begin{array}{cc}
1 & n \\
0 & 1
\end{array}\right)\left(\begin{array}{cc}
1 & 0 \\
1 & 1
\end{array}\right)\right) d n .
$$

It follows from (4.13) that the integral equals

$$
\begin{aligned}
\int_{F_{\mathfrak{p}}-\mathcal{O}_{\mathfrak{p}}} f_{\chi, s, v}\left(\left(\begin{array}{cc}
-1 & -1 \\
1+n & n
\end{array}\right)\right) d n & =\int_{F_{\mathfrak{p}}-\mathcal{O}_{\mathfrak{p}}} f_{\chi, s, v}\left(\left(\begin{array}{cc}
1 / n & * \\
0 & n
\end{array}\right)\left(\begin{array}{cc}
1 & 0 \\
\frac{1+n}{n} & 1
\end{array}\right)\right) d n \\
& =\int_{F_{\mathfrak{p}}-\mathcal{O}_{\mathfrak{p}}} \chi_{1, v}^{-1} \chi_{2, v}(n)|n|^{-(2 s+1)} f_{\chi, s, v}\left(\left(\begin{array}{cc}
1 & 0 \\
\frac{1+n}{n} & 1
\end{array}\right)\right) d n
\end{aligned}
$$

Note that the first equality is obtained by Lemma 2.2.2. Since $\operatorname{val}\left(n^{-1}(n+1)\right)=0$ and since $\chi_{2, v}$ is ramified, we know that $f_{\chi, s, v}\left(\left(\begin{array}{cc}1 & 0 \\ \frac{1+n}{n} & 1\end{array}\right)\right)$ is 0 by Lemma 4.1.1. 
3. Assume that both $\chi_{1, v}$ and $\chi_{2, v}$ are ramified. Similar to the computation of the second assertion, we compute $M_{v, s, e_{p, 1}}$ by computing two integrals. The first integral is

$$
\int_{\mathcal{O}_{\mathfrak{p}}} f_{\chi, s, v}\left(\left(\begin{array}{cc}
0 & -1 \\
1 & 0
\end{array}\right)\left(\begin{array}{cc}
1 & n \\
0 & 1
\end{array}\right)\left(\begin{array}{cc}
1 & 0 \\
\varpi^{e_{\mathfrak{p}, 1}} & 1
\end{array}\right)\right) d n
$$

which is zero by (4.15). The second integral is

$$
\int_{F_{\mathfrak{p}}-\mathcal{O}_{\mathfrak{p}}} f_{\chi, s, v}\left(\left(\begin{array}{cc}
0 & -1 \\
1 & 0
\end{array}\right)\left(\begin{array}{cc}
1 & n \\
0 & 1
\end{array}\right)\left(\begin{array}{cc}
1 & 0 \\
\varpi^{e_{\mathfrak{p}, 1}} & 1
\end{array}\right)\right) d n
$$

It follows from (4.16) that the integral equals

$$
\int_{F_{\mathfrak{p}}-\mathcal{O}_{\mathfrak{p}}} \chi_{1, v}^{-1} \chi_{2, v}(n)|n|^{-(2 s+1)} f_{\chi, s, v}\left(\left(\begin{array}{cc}
1 & 0 \\
\left(1+n \varpi^{e_{\mathfrak{p}, 1}}\right) n^{-1} & 1
\end{array}\right)\right) d n
$$

Note that under our assumption of $\chi_{1, v}$ and $\chi_{2, v}$, we know from Lemma 4.1.1 that

$$
f_{\chi, s, v}\left(\left(\begin{array}{cc}
1 & 0 \\
\left(1+n \varpi^{e_{\mathfrak{p}, 1}}\right) n^{-1} & 1
\end{array}\right)\right) \neq 0 \text { if and only if } \operatorname{val}_{v}\left(\left(1+n \varpi^{e_{\mathfrak{p}, 1}}\right) n^{-1}\right)=e_{\mathfrak{p}, 2} .
$$

When $e_{\mathfrak{p}, 1}>e_{\mathfrak{p}, 2}$, it follows from (4.18) that (4.17) equals

$$
\begin{aligned}
& \int_{\varpi^{-e_{\mathfrak{p}, 2} \mathcal{O}_{\mathfrak{p}}^{\times}}} \chi_{1, v}^{-1} \chi_{2, v}(n)|n|^{-(2 s+1)} f_{\chi, s, v}\left(\left(\begin{array}{cc}
1 & 0 \\
\left(1+n \varpi^{e_{\mathfrak{p}, 1}}\right) n^{-1} & 1
\end{array}\right)\right) d n \\
& =\int_{\varpi^{-e_{\mathfrak{p}, 2} \mathcal{O}_{\mathfrak{p}}^{\times}}} \chi_{1, v}^{-1} \chi_{2, v}(n)|n|^{-(2 s+1)} \chi_{1, v}^{-1}\left(\left(1+n \varpi^{e_{\mathfrak{p}, 1}}\right) n^{-1}\right) d n \\
& =\chi_{2, v}\left(\varpi^{-e_{\mathfrak{p}, 2}}\right)\left|\varpi^{e_{\mathfrak{p}, 2}}\right|^{2 s} \int_{\mathcal{O}_{\mathfrak{p}}^{\times}} \chi_{1, v}^{-1}\left(1+\varpi^{e_{\mathfrak{p}, 1}-e_{\mathfrak{p}, 2}} x\right) \chi_{2, v}(x) d x .
\end{aligned}
$$

By Lemma 2.5.3, we have

$$
\begin{aligned}
& \chi_{2, v}\left(\varpi^{-e_{\mathfrak{p}, 2}}\right)\left|\varpi^{e_{\mathfrak{p}, 2}}\right|^{2 s} \int_{\mathcal{O}_{\mathfrak{p}}^{\times}} \chi_{1, v}^{-1}\left(1+\varpi^{e_{\mathfrak{p}, 1}-e_{\mathfrak{p}, 2}} x\right) \chi_{2, v}(x) d x \\
= & \chi_{2, v}\left(\varpi^{-e_{\mathfrak{p}, 2}}\right)\left|\varpi^{e_{\mathfrak{p}, 2}}\right|^{2 s+2} \sum_{x \in U^{e_{\mathfrak{p}, 2}}} \chi_{2, v}(x) \chi_{1, v}^{-1}\left(1+\varpi^{e_{\mathfrak{p}, 1}-e_{\mathfrak{p}, 2}}+x\right) \\
= & \chi_{2, v}\left(-\varpi^{-e_{\mathfrak{p}, 2}}\right)\left|\varpi^{e_{\mathfrak{p}, 2}}\right|^{2 s+2} \sum_{x \in U^{\boldsymbol{e}_{\mathbf{p}, 2}}} \chi_{2, v}(x) \chi_{1, v}^{-1}\left(1-\varpi^{e_{\mathfrak{p}, 1}-e_{\mathfrak{p}, 2}}+x\right) .
\end{aligned}
$$

This proves the assertion (3.1). 
When $e_{\mathfrak{p}, 2}>e_{\mathfrak{p}, 1}$, it follows from (4.18) that (4.17) equals

$$
\begin{aligned}
& \int_{\varpi^{-e_{\mathfrak{p}, 1}}\left(-1+\varpi^{\left.e_{\mathfrak{p}, 2}-e_{\mathfrak{p}, 1} \mathcal{O}_{\mathfrak{p}}^{\times}\right)}\right.} \chi_{1, v}^{-1} \chi_{2, v}(n)|n|^{-(2 s+1)} f_{\chi, s, v}\left(\left(\begin{array}{cc}
1 & 0 \\
\left(1+n \varpi^{e_{\mathfrak{p}, 1}}\right) n^{-1} & 1
\end{array}\right)\right) d n \\
& =\int_{\varpi^{-e_{\mathfrak{p}, 1}}\left(-1+\varpi^{\left.e_{\mathfrak{p}, 2}-e_{\mathfrak{p}, 1} \mathcal{O}_{\mathfrak{p}}^{\times}\right)}\right.} \chi_{1, v}^{-1} \chi_{2, v}(n)|n|^{-(2 s+1)} \chi_{1, v}^{-1}\left(\left(1+n \varpi^{e_{\mathfrak{p}, 1}}\right) n^{-1}\right) d n \\
& =\chi_{1, v}^{-1}\left(\varpi^{e_{\mathfrak{p}, 2}-e_{\mathfrak{p}, 1}}\right) \chi_{2, v}\left(\varpi^{-e_{\mathfrak{p}, 1}}\right)\left|\varpi^{e_{\mathfrak{p}, 1}}\right|^{2 s}\left|\varpi^{e_{\mathfrak{p}, 2}-e_{\mathfrak{p}, 1}}\right| \int_{\mathcal{O}_{\mathfrak{p}}^{\times}} \chi_{2, v}\left(-1+\varpi^{e_{\mathfrak{p}, 2}-e_{\mathfrak{p}, 1}} x\right) \chi_{1, v}^{-1}(x) d x \\
& =\chi_{1, v}^{-1}\left(\varpi^{e_{\mathfrak{p}, 2}-e_{\mathfrak{p}, 1}}\right) \chi_{2, v}\left(\varpi^{-e_{\mathfrak{p}, 1}}\right)|\varpi|^{2 s e_{\mathfrak{p}, 1}+e_{\mathfrak{p}, 2}} \sum_{x \in U_{\mathfrak{p}}^{\left(e_{\mathfrak{p}, 1}\right)}} \chi_{1, v}^{-1}(x) \chi_{2, v}\left(-1+\varpi^{e_{\mathfrak{p}, 2}-e_{\mathfrak{p}, 1}} x\right) .
\end{aligned}
$$

Note that the first equality is obtained by Lemma 4.1 .1 and the last equality is obtained by Lemma 2.5.3. This proves the assertion (3.2).

Now we assume that $e_{\mathfrak{p}, 1}=e_{\mathfrak{p}, 2}$. It follows from (4.18) that (4.17) equals

$$
\sum_{k=e_{\mathrm{p}, 1}}^{\infty} \int_{\varpi^{-k} \mathcal{O}_{\boldsymbol{p}}^{\times}} \chi_{1, v}^{-1} \chi_{2, v}(n)|n|^{-(2 s+1)} f_{\chi, s, v}\left(\left(\begin{array}{cc}
1 & 0 \\
\left(1+n \varpi^{e_{\mathfrak{p}, 1}}\right) n^{-1} & 1
\end{array}\right)\right) d n .
$$

We will compute this integral by separating it into two parts. One part is when $k=e_{\mathfrak{p}, 1}$ and the other one is when $k \geq e_{\mathfrak{p}, 1}+1$. When $k=e_{\mathfrak{p}, 1}$, we have

$$
\begin{aligned}
& \int_{\varpi^{-e_{\mathfrak{p}, 1} \mathcal{O}_{\mathfrak{p}}^{\times}}} \chi_{1, v}^{-1} \chi_{2, v}(n)|n|^{-(2 s+1)} f_{\chi, s, v}\left(\left(\begin{array}{cc}
1 & 0 \\
\left(1+n \varpi^{e_{\mathfrak{p}, 1}}\right) n^{-1} & 1
\end{array}\right)\right) d n \\
& =\sum_{\substack{j \in\left(\mathcal{O}_{\mathfrak{p}} / \varpi\right)^{\times} \\
j \neq-1}} \int_{\varpi^{-e_{\mathfrak{p}, 1}\left(j+\varpi \mathcal{O}_{\mathfrak{p}}\right)}} \chi_{1, v}^{-1}\left(1+n \varpi^{e_{\mathfrak{p}, 1}}\right) \chi_{2, v}(n)|n|^{-(2 s+1)} d n \\
& =\chi_{2, v}\left(\varpi^{-e_{\mathfrak{p}, 1}}\right)|\varpi|^{2 s e_{\mathfrak{p}, 1}} \int_{\mathcal{O}_{\mathfrak{p}}^{\times}-\left\{-1+\varpi \mathcal{O}_{\mathfrak{p}}\right\}} \chi_{1, v}^{-1}(1+x) \chi_{2, v}(x) d x .
\end{aligned}
$$

By Lemma 2.5.3, we know that

$$
\begin{aligned}
& \chi_{2, v}\left(\varpi^{-e_{\mathfrak{p}, 1}}\right)|\varpi|^{2 s e_{\mathfrak{p}, 1}} \int_{\mathcal{O}_{\mathfrak{p}}^{\times}-\left\{-1+\varpi \mathcal{O}_{\mathfrak{p}}\right\}} \chi_{1, v}^{-1}(1+x) \chi_{2, v}(x) d x \\
& =\chi_{2, v}\left(\varpi^{-e_{\mathfrak{p}, 1}}\right)|\varpi|^{2 s e_{\mathfrak{p}, 1}+e_{\mathfrak{p}, 1}} \sum_{\substack{x \in U_{\mathfrak{p}}^{\left(e_{\mathfrak{p}, 1}\right)} \\
x \notin-1+\varpi \mathcal{O}_{\mathfrak{p}}}} \chi_{2, v}(x) \chi_{1, v}^{-1}(1+x) \\
& =\chi_{2, v}\left(-\varpi^{-e_{\mathfrak{p}, 1}}\right)|\varpi|^{e_{\mathfrak{p}, 1}(2 s+1)} J_{1}\left(\chi_{2, v}, \chi_{1, v}^{-1}, \varpi^{e_{\mathfrak{p}, 1}}\right) .
\end{aligned}
$$


When $k \geq e_{\mathfrak{p}, 1}+1$, we have

$$
\begin{aligned}
& \sum_{k=e_{\mathfrak{p}, 1+1}}^{\infty} \int_{\varpi^{-k} \mathcal{O}_{\mathfrak{p}}^{\times}} \chi_{1, v}^{-1} \chi_{2, v}(n)|n|^{-(2 s+1)} f_{\chi, s, v}\left(\left(\begin{array}{cc}
1 & 0 \\
\left(1+n \varpi^{e_{\mathfrak{p}, 1}}\right) n^{-1} & 1
\end{array}\right)\right) d n \\
= & \sum_{k=e_{\mathfrak{p}, 1}+1}^{\infty} \int_{\varpi^{-k} \mathcal{O}_{\mathfrak{p}}^{\times}} \chi_{1, v}^{-1}\left(1+\varpi^{e_{\mathfrak{p}, 1}} n\right) \chi_{2, v}(n)|n|^{-(2 s+1)} d n \\
= & \sum_{k=e_{\mathfrak{p}, 1}+1}^{\infty} \chi_{2, v}\left(\varpi^{-k}\right)|\varpi|^{2 s k} \int_{\mathcal{O}_{\mathfrak{p}}^{\times}} \chi_{1, v}^{-1}\left(1+\varpi^{e_{\mathfrak{p}, 1}-k} x\right) \chi_{2, v}(x) d x \\
= & \sum_{k=e_{\mathfrak{p}, 1}+1}^{\infty} \chi_{1, v}^{-1}\left(\varpi^{e_{\mathfrak{p}, 1}-k}\right) \chi_{2, v}\left(\varpi^{-k}\right)|\varpi|^{2 s k} \int_{\mathcal{O}_{\mathfrak{p}}^{\times}} \chi_{1, v}^{-1}\left(\varpi^{k-e_{\mathfrak{p}, 1}}+x\right) \chi_{2, v}(x) d x .
\end{aligned}
$$

By Lemma 2.5.3, we have

$$
\begin{aligned}
& \sum_{k=e_{\mathfrak{p}, 1}+1}^{\infty} \chi_{1, v}^{-1}\left(\varpi^{e_{\mathfrak{p}, 1}-k}\right) \chi_{2, v}\left(\varpi^{-k}\right)|\varpi|^{2 s k} \int_{\mathcal{O}_{\mathfrak{p}}^{\times}} \chi_{1, v}^{-1}\left(\varpi^{k-e_{\mathfrak{p}, 1}}+x\right) \chi_{2, v}(x) d x \\
= & \sum_{k=e_{\mathfrak{p}, 1}+1}^{\infty} \chi_{1, v}^{-1}\left(\varpi^{e_{\mathfrak{p}, 1}-k}\right) \chi_{2, v}\left(\varpi^{-k}\right)|\varpi|^{2 s k} \sum_{x \in U_{\mathfrak{p}}^{\left(e_{\mathfrak{p}, 1}\right)}} \chi_{2, v}(x) \int_{1+\varpi_{\mathfrak{p}, 1}^{e_{\mathfrak{p}}}} \chi_{1, v}^{-1}\left(\varpi^{k-e_{\mathfrak{p}, 1}}+x y\right) d y \\
= & \sum_{k=e_{\mathfrak{p}, 1}+1}^{\infty} \chi_{1, v}^{-1}\left(\varpi^{e_{\mathfrak{p}, 1}-k}\right) \chi_{2, v}\left(\varpi^{-k}\right)|\varpi|^{2 s k+e_{\mathfrak{p}, 1}} \sum_{x \in U_{\mathfrak{p}}^{\left(e_{\mathfrak{p}, 1}\right)}} \chi_{2, v}(x) \chi_{1, v}^{-1}\left(\varpi^{k-e_{\mathfrak{p}, 1}}+x\right) \\
= & \sum_{k=e_{\mathfrak{p}, 1}+1}^{2 e_{\mathfrak{p}, 1}-1} \chi_{1, v}^{-1}\left(\varpi^{e_{\mathfrak{p}, 1}-k}\right) \chi_{2, v}\left(\varpi^{-k}\right)|\varpi|^{2 s k+e_{\mathfrak{p}, 1}} \sum_{x \in U_{\mathfrak{p}}^{\left(e_{\mathfrak{p}, 1}\right)}} \chi_{2, v}(x) \chi_{1, v}^{-1}\left(\varpi^{k-e_{\mathfrak{p}, 1}}+x\right) \\
& +\sum_{k=2 e_{\mathfrak{p}, 1}}^{\infty} \chi_{1, v}^{-1}\left(\varpi^{e_{\mathfrak{p}, 1}-k}\right) \chi_{2, v}\left(\varpi^{-k}\right)|\varpi|^{2 s k+e_{\mathfrak{p}, 1}} \sum_{x \in U_{\mathfrak{p}}^{\left(e_{\mathfrak{p}, 1}\right)}} \chi_{2, v}(x) \chi_{1, v}^{-1}(x) .
\end{aligned}
$$

To sum up, we have shown that if $e_{\mathfrak{p}, 1}=e_{\mathfrak{p}, 2}$, then

$$
\begin{aligned}
& \int_{F_{\mathfrak{p}}-\mathcal{O}_{\mathfrak{p}}} \chi_{1, v}^{-1} \chi_{2, v}(n)|n|^{-(2 s+1)} f_{\chi, s, v}\left(\left(\begin{array}{cc}
1 & 0 \\
\left(1+n \varpi^{e_{\mathfrak{p}, 1}}\right) n^{-1} & 1
\end{array}\right)\right) d n \\
= & \chi_{2, v}\left(-\varpi^{-e_{\mathfrak{p}, 1}}\right)|\varpi|^{e_{\mathfrak{p}, 1}(2 s+1)} J_{1}\left(\chi_{2, v}, \chi_{1, v}, \varpi^{e_{\mathfrak{p}, 1}}\right) \\
& +\sum_{k=e_{\mathfrak{p}, 1}+1}^{2 e_{\mathfrak{p}, 1}-1} \chi_{1, v}^{-1}\left(\varpi^{e_{\mathfrak{p}, 1}-k}\right) \chi_{2, v}\left(\varpi^{-k}\right)|\varpi|^{2 s k+e_{\mathfrak{p}, 1}} \sum_{x \in U_{\mathfrak{p}}^{\left(e_{\mathfrak{p}, 1}\right)}} \chi_{2, v}(x) \chi_{1, v}^{-1}\left(\varpi^{k-e_{\mathfrak{p}, 1}}+x\right) \\
& +\sum_{k=2 e_{\mathfrak{p}, 1}}^{\infty} \chi_{1, v}^{-1}\left(\varpi^{e_{\mathfrak{p}, 1}-k}\right) \chi_{2, v}\left(\varpi^{-k}\right)|\varpi|^{2 s k+e_{\mathfrak{p}, 1}} \sum_{x \in U_{\mathfrak{p}}^{\left(e_{\mathfrak{p}, 1}\right)}} \chi_{2, v}(x) \chi_{1, v}^{-1}(x) .
\end{aligned}
$$


If $\chi_{1, v}^{-1} \chi_{2, v}$ is a primitive character, then by [Jun, Lemma 2.3], we see that

$$
\sum_{x \in U_{\mathfrak{p}}^{\left(e_{\mathfrak{p}, 1}\right)}} \chi_{2, v}(x) \chi_{1, v}^{-1}\left(\varpi^{k-e_{\mathfrak{p}, 1}}+x\right)=0
$$

for all $k \geq e_{\mathfrak{p}, 1}$ and

$$
\sum_{x \in U_{\mathfrak{p}}^{\left(e_{\mathfrak{p}, 1}\right)}} \chi_{2, v}(x) \chi_{1, v}^{-1}(x)=0 .
$$

Thus the assertion (3.3) follows. Similarly, the assertions (3.4) and (3.5) follow from Lemma 2.3 and Lemma 2.4 in [Jun]. This completes the proof.

\subsection{Archimedean sections}

We define an infinite section $f_{s, \infty}: \mathrm{GL}_{2}(\mathbb{R}) \longrightarrow \mathbb{C}^{\times}$by

$$
f_{s, \infty}\left(\left(\begin{array}{cc}
a_{1} & b \\
0 & a_{2}
\end{array}\right) k_{\theta}\right)=\left|\frac{a_{1}}{a_{2}}\right|^{s+\frac{1}{2}} \cdot j\left(k_{\theta}, i\right)^{-k}\left|j\left(k_{\theta}, i\right)\right|^{k-2 s-1}
$$

for $a_{1}, a_{2} \in \mathbb{R}$ and $k_{\theta} \in \mathrm{SO}_{2}(\mathbb{R})$. Here $j\left(k_{\theta}, i\right)$ is the automorphic factor. Equivalently, one can define $f_{s, \infty}$ by

$$
f_{s, \infty}(g)=j(g, i)^{-k}|j(g, i)|^{k-2 s-1}|\operatorname{det}(g)|^{s+\frac{1}{2}}
$$

for $g \in \mathrm{GL}_{2}(\mathbb{R})$. Recall that for $\beta \in F$ and $g \in \mathrm{GL}_{2}(\mathbb{R})$, the integral

$$
\int_{\mathbb{R}} f_{s, \infty}\left(\left(\begin{array}{cc}
0 & -1 \\
1 & 0
\end{array}\right)\left(\begin{array}{cc}
1 & n \\
0 & 1
\end{array}\right) g\right) \psi_{\infty}(-\beta n) d n
$$

converges absolutely when $\mathrm{re}(s)$ is big enough and has analytic continuation for all $s$.

Now, we assume that $\mathrm{re}(s)$ is big enough so that the above integral converges absolutely. For the proof of Lemma 4.2.1, we will first prove the assertions when re(s) is big enough and obtain the result for all $s$ by analytic continuation. 
Lemma 4.2.1. For $z=x+i y$, we set $g_{z}=\left(\begin{array}{ll}y & x \\ 0 & 1\end{array}\right)$. Then

$$
\begin{aligned}
& \int_{\mathbb{R}} f_{s, \infty}\left(\left(\begin{array}{cc}
0 & -1 \\
1 & 0
\end{array}\right)\left(\begin{array}{cc}
1 & n \\
0 & 1
\end{array}\right) g_{z}\right) \psi_{\infty}(-\beta n) d n \text { if } \beta=0 \\
&= \begin{cases}y^{\frac{1}{2}-s} i^{-k} 2^{-2 s+1} \pi \frac{\Gamma(2 s)}{\Gamma\left(s+\frac{1+k}{2}\right) \Gamma\left(s+\frac{1-k}{2}\right)} & \text { if } \beta>0 \\
i^{-k} 2^{k} \pi^{s+\frac{1-k}{2}} \Gamma\left(s+\frac{1-k}{2}\right)^{-1} y^{-k / 2} \beta^{s+\frac{k-1}{2}} e^{2 \pi i \beta z} \omega\left(4 \pi \beta y ; s+\frac{1+k}{2}, s+\frac{1-k}{2}\right) \\
i^{-k} 2^{-k} \pi^{s+\frac{1-k}{2}} \Gamma\left(s+\frac{1+k}{2}\right)^{-1}|\beta|^{s-\frac{1+k}{2}} e^{2 \pi i|\beta| z} \omega\left(4 \pi|\beta| y ; s+\frac{1-k}{2}, s+\frac{1+k}{2}\right) & \text { if } \beta<0,\end{cases}
\end{aligned}
$$

where $\omega(z ; a, b)$ is defined by

$$
\omega(z ; a, b)=z^{b} \Gamma(b)^{-1} \int_{0}^{\infty} e^{z x}(x+1)^{a-1} x^{b-1} d x
$$

for $z \in \mathbb{C}$ with re $z>0$, and $a, b \in \mathbb{C}$, which is a holomorphic function on $\mathbf{H} \times \mathbb{C}^{2}$ [H2, p. 288]. Here $\Gamma(z)$ is the Gamma function for $z \in \mathbb{C}$ and $\mathbf{H}$ is the complex upper half plane. In particular, by taking $s \rightarrow \frac{1-k}{2}$, we obtain

$$
\left[\int_{\mathbb{R}} f_{s, \infty}\left(\left(\begin{array}{cc}
0 & -1 \\
1 & 0
\end{array}\right)\left(\begin{array}{cc}
1 & n \\
0 & 1
\end{array}\right) g_{z}\right) \psi_{\infty}(-\beta n) d n\right]_{s=\frac{1-k}{2}}= \begin{cases}\frac{1}{2} C_{\infty}(k) & \text { if } \beta=0 \\
C_{\infty}(k) e^{2 \pi i \beta z} & \text { if } \beta>0 \\
0 & \text { if } \beta<0,\end{cases}
$$

where $C_{\infty}(k)=i^{-k} 2^{k} \pi y^{k / 2}$

Proof. Since the measure $d n$ is additive, we have

$$
\int_{\mathbb{R}} f_{s, \infty}\left(\left(\begin{array}{cc}
0 & -1 \\
1 & 0
\end{array}\right)\left(\begin{array}{cc}
1 & n \\
0 & 1
\end{array}\right) g_{z}\right) \psi_{\infty}(-\beta n) d n=\int_{\mathbb{R}} f_{s, \infty}\left(\left(\begin{array}{cc}
0 & -1 \\
y & n
\end{array}\right)\right) \psi_{\infty}(-\beta(n-x)) d n
$$

which equals

$$
\psi_{\infty}(\beta x) y^{s+\frac{1}{2}} \int_{\mathbb{R}}(n+i y)^{-k}|n+i y|^{-2\left(s-\frac{k-1}{2}\right)} \psi(-\beta n) d n .
$$

Then the first assertion follows from the lemma below. 
For the second assertion, when $\beta=0$, the assertion follows from the observation that

$$
\left.\frac{\Gamma(2 s)}{\Gamma\left(s+\frac{1+k}{2}\right) \Gamma\left(s+\frac{1-k}{2}\right)}\right|_{s=\frac{1-k}{2}}=\frac{1}{2} .
$$

When $\beta \neq 0$, the assertion follows from direct computation and the fact [H2, p. 288] that $\omega(z ; 1, \alpha)=$

1.

Lemma 4.2.2. For any $\alpha, \beta \in \mathbb{C}$ and $h, y \in \mathbb{R}$, we have

$$
\begin{aligned}
& \int_{\mathbb{R}}(x+i y)^{-\alpha}|x+i y|^{-2 \beta} \psi(-h x) d x \\
= & \begin{cases}i^{-\alpha} 2^{1-\alpha-2 \beta} \pi \Gamma(\alpha+\beta)^{-1} \Gamma(\beta)^{-1} \Gamma(\alpha+2 \beta-1) y^{1-\alpha-2 \beta} & \text { if } h=0 \\
i^{-\alpha}(2 \pi)^{\alpha+\beta} \Gamma(\alpha+\beta)^{-1}(2 y)^{-\beta} h^{\alpha+\beta-1} e^{-2 \pi h y} \omega(4 \pi h y ; \alpha+\beta, \beta) & \text { if } h>0 \\
i^{-\alpha}(2 \pi)^{\beta} \Gamma(\beta)^{-1}(2 y)^{-\alpha-\beta}|h|^{\beta-1} e^{-2 \pi|h| y} \omega(4 \pi|h| y ; \beta, \alpha+\beta) & \text { if } h<0 .\end{cases}
\end{aligned}
$$

For a proof, see [H2, §9.2 Lemma 3].

\subsection{More on Eisenstein series}

Recall that we fix two primitive narrow ray class characters $\chi_{1}$ and $\chi_{2}$ of conductor $\mathfrak{n}_{1}$ and $\mathfrak{n}_{2}$, respectively, which satisfy the same conditions as those in Proposition 3.3.3. Also, recall that we write $\mathfrak{n}_{1} \mathfrak{n}_{2}=\mathfrak{n}$ and assume that it is coprime to the ideal generated by $t_{\lambda} \mathfrak{D}$. The Eisenstein series associated to the section $f_{\chi, s}=\bigotimes_{v \mid \infty} f_{s, \infty} \otimes \bigotimes_{v<\infty} f_{\chi, s, v}$ is defined by

$$
E\left(f_{\chi, s}, g\right)=\sum_{\gamma \in B(F) \backslash \mathrm{GL}_{2}(F)} f_{\chi, s}(\gamma g)
$$

for all $g \in \mathrm{GL}_{2}\left(\mathbb{A}_{F}\right)$, which converges absolutely when $r e(s)>1 / 2[\mathrm{~B}$, Proposition 3.7.2]. Recall that for a narrow ray class character $\chi$, the $L$-function $L(s, \chi)$ was defined in Proposition 3.3.3. The 
partial $L$-function $L^{\mathfrak{n}}\left(s, \chi_{1} \chi_{2}^{-1}\right)$ is defined as

$$
L^{\mathfrak{n}}\left(s, \chi_{1} \chi_{2}^{-1}\right)=\prod_{\substack{\mathfrak{q} \mid \mathfrak{n}, \mathfrak{q} \nmid \operatorname{cond}\left(\chi_{1} \chi_{2}^{-1}\right)}}\left(1-\chi_{1} \chi_{2}^{-1}(\mathfrak{q}) N(\mathfrak{q})^{-s}\right) L\left(s, \chi_{1} \chi_{2}^{-1}\right) .
$$

The normalized Eisenstein series $L^{\mathfrak{n}}\left(2 s+1, \chi_{1} \chi_{2}^{-1}\right) E\left(f_{\chi, s}, g\right)$ has meromorphic continuation to all $s$ except that it has a pole at $s=\frac{1}{2}$ if $\chi_{1}=\chi_{2}$ [B, Theorem 3.7.1]. Since the local epsilon factors $\varepsilon_{v}\left(2 s+1, \chi_{2}^{-1}, \psi_{v}\right)$ have neither zeros nor poles (see Section 4.1), the Eisenstein series

$$
E\left(\chi_{1}, \chi_{2}\right)(z, s, g)=\frac{C_{\infty}(k)^{-d} L^{\mathfrak{n}}\left(2 s+1, \chi_{1} \chi_{2}^{-1}\right)}{\prod_{v \mid \mathfrak{n}_{2}} \varepsilon_{v}\left(2 s+1, \chi_{2}^{-1}, \psi_{v}\right)} E\left(f_{\chi, s}, g_{z} g\right)
$$

can only possibility have a pole at $s=\frac{1}{2}$ as well. The classical Eisenstein series $E_{k}\left(\chi_{1}, \chi_{2}\right)(z, g) \in$ $M_{k}\left(\mathfrak{n}, \chi_{1} \chi_{2} ; \mathbb{C}\right)$ is defined by

$$
E_{k}\left(\chi_{1}, \chi_{2}\right)(z, g):=E\left(\chi_{1}, \chi_{2}\right)\left(z, \frac{1-k}{2}, g\right)=\left.\frac{C_{\infty}(k)^{-d} L^{\mathfrak{n}}\left(2 s+1, \chi_{1} \chi_{2}^{-1}\right)}{\prod_{v \mid \mathfrak{n}_{2}} \varepsilon_{v}\left(2 s+1, \chi_{2}^{-1}, \psi_{v}\right)} E\left(f_{\chi, s}, g_{z} g\right)\right|_{s=\frac{1-k}{2}}
$$

for $z \in \mathbf{H}^{d}$ and $g \in \mathrm{GL}_{2}\left(\mathbb{A}_{F, f}\right)$, where $C_{\infty}(k)$ was defined in Lemma 4.2.1.

In the remainder of this section, we will assume that $\operatorname{re}(s)>1 / 2$. The Fourier expansion of $E\left(f_{\chi, s}, g_{z} g\right)$ is given by $[\mathrm{B}, \S 3.7]$

$$
E\left(f_{\chi, s}, g_{z} g\right)=\sum_{\beta \in F} c_{\beta}\left(E\left(f_{\chi, s}, g_{z} g\right)\right)
$$

where $c_{\beta}\left(E\left(f_{\chi, s}\left(g_{z} \gamma\right)\right)\right)$ is defined as

$$
c_{\beta}\left(E\left(f_{\chi, s}, g_{z} g\right)\right):=\int_{F \backslash \mathbb{A}_{F}} E\left(f_{\chi, s},\left(\begin{array}{cc}
1 & n \\
0 & 1
\end{array}\right) g_{z} g\right) \psi(-\beta n) d n .
$$

Here $d n=\otimes_{v} d n_{p}$ is the self-dual Haar measure defined as follows. For each finite place $\mathfrak{p}, d n_{\mathfrak{p}}$ is the normalized Haar measure such that the volume of $\mathcal{O}_{\mathfrak{p}}$ is 1 , and for the infinite place the Haar measure $d n_{\infty}$ is normalized such that the volume of $\mathbb{R} / \mathbb{Z}$ is 1 . The number $c_{0}\left(E\left(f_{\chi, s}, g_{z} g\right)\right)$ is called the constant term of $E\left(f_{\chi, s}, g_{z} g\right)$ at the cusp $g$. 
Recall that the Bruhat decomposition for $\mathrm{GL}_{2}(F)$ is

$$
\mathrm{GL}_{2}(F)=B(F) \coprod B(F)\left(\begin{array}{cc}
0 & -1 \\
1 & 0
\end{array}\right), N(F)
$$

which implies that

$$
E\left(f_{\chi, s}, g_{z} g\right)=f_{\chi, s}\left(g_{z} g\right)+\sum_{n \in N(F)} f_{\chi, s}\left(\left(\begin{array}{cc}
0 & -1 \\
1 & 0
\end{array}\right) n g_{z} g\right)
$$

Thus, one can simplify the integral (4.19) as

$$
f_{\chi, s}\left(g_{z} g\right)+\int_{\mathbb{A}_{F}} f_{\chi, s}\left(\left(\begin{array}{cc}
0 & -1 \\
1 & 0
\end{array}\right)\left(\begin{array}{cc}
1 & n \\
0 & 1
\end{array}\right) g_{z} g\right) d n
$$

if $\beta=0$, and

$$
\int_{\mathbb{A}_{F}} f_{\chi, s}\left(\left(\begin{array}{cc}
0 & -1 \\
1 & 0
\end{array}\right)\left(\begin{array}{cc}
1 & n \\
0 & 1
\end{array}\right) g_{z} g\right) \psi(-\beta n) d n .
$$

if $\beta \neq 0$. It follows from Lemma 4.2.1 that the integral is 0 if $\beta$ is not totally positive. Moreover, it follows from Tate's thesis [Cas-Fro] that for all $\beta \in F$, we have

$$
\int_{\mathbb{A}_{F}} f_{\chi, s}\left(\left(\begin{array}{cc}
0 & -1 \\
1 & 0
\end{array}\right)\left(\begin{array}{cc}
1 & n \\
0 & 1
\end{array}\right) g_{z} g\right) \psi(-\beta n) d n=\prod_{v \leq \infty} \int_{F_{v}} f_{\chi, s, v}\left(\left(\begin{array}{cc}
0 & -1 \\
1 & 0
\end{array}\right)\left(\begin{array}{cc}
1 & n \\
0 & 1
\end{array}\right) g_{z} g\right) \psi_{v}\left(-\beta n_{v}\right) d n_{v}
$$

Again by Lemma 4.2.1, one obtains

$$
\prod_{v \mid \infty} \int_{F_{v}} f_{\chi, s, v}\left(\left(\begin{array}{cc}
0 & -1 \\
1 & 0
\end{array}\right)\left(\begin{array}{cc}
1 & n \\
0 & 1
\end{array}\right) g_{z}\right) \psi_{v}\left(-\beta n_{v}\right) d n_{v}=C_{\infty}(k)^{d} e^{2 \pi i \operatorname{Tr}(\beta z)}
$$

To sum up, we have seen that the $\beta$-th Fourier coefficient $c_{\beta}\left(E_{k}\left(\chi_{1}, \chi_{2}\right)(z, g)\right)$ of $E_{k}\left(\chi_{1}, \chi_{2}\right)(z, g)$ is given by

$$
\left[\frac{L^{\mathfrak{n}}\left(2 s+1, \chi_{1} \chi_{2}^{-1}\right)}{\prod_{v \mid \mathfrak{n}_{2}} \varepsilon_{v}\left(2 s+1, \chi_{2}^{-1}, \psi_{v}\right)} \prod_{v<\infty} \int_{F_{v}} f_{\chi, s, v}\left(\left(\begin{array}{cc}
0 & -1 \\
1 & 0
\end{array}\right)\left(\begin{array}{cc}
1 & n \\
0 & 1
\end{array}\right) g\right) \psi_{v}\left(-\beta n_{v}\right) d n_{v}\right]_{s=\frac{1-k}{2}}
$$

and the constant term $c_{0}\left(E_{k}\left(\chi_{1}, \chi_{2}\right)(z, g)\right)$ is given by

$$
\left[\frac{C_{\infty}(k)^{-d} L^{\mathfrak{n}}\left(2 s+1, \chi_{1} \chi_{2}^{-1}\right)}{\prod_{v \mid \mathfrak{n}_{2}} \varepsilon_{v}\left(2 s+1, \chi_{2}^{-1}, \psi_{v}\right)}\left(f_{\chi, s}\left(g_{z} g\right)+\int_{\mathbb{A}_{F}} f_{\chi, s}\left(\left(\begin{array}{cc}
0 & -1 \\
1 & 0
\end{array}\right)\left(\begin{array}{cc}
1 & n \\
0 & 1
\end{array}\right) g_{z} g\right) \psi(-\beta n) d n\right)\right]_{s=\frac{1-k}{2}}
$$


Note that when $\beta=0$, the above local integrals are $M_{v} f_{\chi, s, v}(g)$, as defined in Section 4.1.

Now we compute the Fourier coefficient at the cusp $x_{\lambda}^{-1}=\left(\begin{array}{cc}\left(t_{\lambda} \delta\right)^{-1} & 0 \\ 0 & 1\end{array}\right)$, where the element $\delta \in$ $\mathbb{A}_{F, f}$ such that $\delta \mathcal{O}_{F}=\mathfrak{D}$ was defined in Section 3.2. Note that all of the Fourier coefficients in Proposition 4.3.1 are unnormalized. One can multiply $N\left(t_{\lambda} \mathfrak{D}\right)^{-k / 2}$ to obtain the normalized Fourier coefficients.

Proposition 4.3.1. Let the notation be as above. Suppose that $\chi_{1}$ and $\chi_{2}$ satisfy the assumption in Proposition 3.3.3. Then we have

$$
c_{0}\left(E_{k}\left(\chi_{1}, \chi_{2}\right)\left(z, x_{\lambda}^{-1}\right)\right)= \begin{cases}2^{-d} N\left(t_{\lambda} \mathfrak{D}\right)^{k / 2} \chi_{2}^{-1}\left(t_{\lambda} \mathfrak{D}\right) L\left(1-k, \chi_{1} \chi_{2}^{-1}\right) & \text { if } \mathfrak{n}_{2}=1 \\ 0 & \text { otherwise }\end{cases}
$$

for $\lambda=1, \ldots, h^{+}$. Moreover, for any integral ideal $\mathfrak{m}$ of $F$, we have

$$
c_{\beta}\left(E_{k}\left(\chi_{1}, \chi_{2}\right)\left(z, x_{\lambda}^{-1}\right)\right)=N\left(t_{\lambda} \mathfrak{D}\right)^{k / 2} \sum_{\mathfrak{a} \mid \mathfrak{m}} \chi_{1}(\mathfrak{a}) \chi_{2}\left(\frac{\mathfrak{m}}{\mathfrak{a}}\right) N(\mathfrak{a})^{k-1}
$$

where $\beta \in F^{+}$satisfies $\mathfrak{m}=\left(t_{\lambda} \delta\right)^{-1} \beta \mathcal{O}_{F}$. In particular, $E_{k}\left(\chi_{1}, \chi_{2}\right)(z, g)$ is the same as the Eisenstein series in Proposition 3.3.3.

ProOF. We first compute $c_{0}\left(E_{k}\left(\chi_{1}, \chi_{2}\right)\left(z, x_{\lambda}^{-1}\right)\right)$. Under the assumption in Proposition 3.3.3, we know that the infinite part of $\chi_{1} \chi_{2}^{-1}$ is $\operatorname{sgn}^{k}$, which implies that $L^{\mathfrak{n}}\left(2-k, \chi_{1} \chi_{2}^{-1}\right)$ is zero. Thus, we only have to compute the second term in (4.21). If $\mathfrak{n}_{2} \neq 1$, it is 0 by Lemma $4.1 .3(3)$. Now we suppose that $\mathfrak{n}_{2}=1$. It follows from Lemma 4.2.1 that

$$
\left[\prod_{v \mid \infty} \int_{F_{v}} f_{\chi, s, v}\left(\left(\begin{array}{cc}
0 & -1 \\
1 & 0
\end{array}\right)\left(\begin{array}{cc}
1 & n \\
0 & 1
\end{array}\right) g_{z}\right) d n_{v}\right]_{s=\frac{1-k}{2}}=\frac{C_{\infty}(k)^{d}}{2^{d}}
$$

Moreover, by Lemma 4.1.2, Lemma 4.1.3, and the uniqueness of meromorphic continuation of $L$ - 
functions, we obtain

$$
\begin{aligned}
& {\left[\prod_{v<\infty} \int_{F_{v}} f_{\chi, s, v}\left(\left(\begin{array}{cc}
0 & -1 \\
1 & 0
\end{array}\right)\left(\begin{array}{cc}
1 & n_{v} \\
0 & 1
\end{array}\right) x_{\lambda}^{-1}\right) d n_{v}\right]_{s=\frac{1-k}{2}} } \\
= & {\left[\left(\prod_{v<\infty} \chi_{2, v}\left(t_{\lambda} \delta\right) N_{F_{v} / \mathbb{Q}_{p}}\left(t_{\lambda} \delta\right)^{k / 2}\right) \frac{L\left(2 s, \chi_{1} \chi_{2}^{-1}\right)}{L\left(2 s+1, \chi_{1} \chi_{2}^{-1}\right)}\right]_{s=\frac{1-k}{2}} } \\
= & \chi_{2}\left(t_{\lambda} \mathfrak{D}\right) N\left(t_{\lambda} \mathfrak{D}\right)^{k / 2} \frac{L\left(1-k, \chi_{1} \chi_{2}^{-1}\right)}{L\left(2-k, \chi_{1} \chi_{2}^{-1}\right)} .
\end{aligned}
$$

Thus the first assertion follows from (4.22). Moreover, we see that the constant term of $E_{k}\left(\chi_{1}, \chi_{2}\right)\left(g_{z}, x_{\lambda}^{-1}\right)$ coincides with $C_{\lambda}\left(E_{k}\left(\chi_{1}, \chi_{2}\right)\right)$ in Proposition 3.3.3.

Next, we compute $c_{\beta}\left(E_{k}\left(\chi_{1}, \chi_{2}\right)\left(z, x_{\lambda}^{-1}\right)\right)$ for $\beta \in F^{+}$satisfying $\mathfrak{m}=\left(t_{\lambda} \delta\right)^{-1} \beta \mathcal{O}_{F}$. To show $E_{k}\left(\chi_{1}, \chi_{2}\right)(z, g)$ coincides with the Eisenstein series in Proposition 3.3.3, it suffices to show

$$
c_{\beta}\left(E_{k}\left(\chi_{1}, \chi_{2}\right)\left(z, x_{\lambda}^{-1}\right)\right)=N\left(t_{\lambda} \mathfrak{D}\right)^{k / 2} \sum_{\mathfrak{a} \mid \mathfrak{p}^{\alpha}} \chi_{1}(\mathfrak{a}) \chi_{2}\left(\frac{\mathfrak{p}^{\alpha}}{\mathfrak{a}}\right) N(\mathfrak{a})^{k-1}
$$

where $\beta \in F^{+}$satisfies $\mathfrak{p}^{\alpha}=\left(t_{\lambda} \delta\right)^{-1} \beta \mathcal{O}_{F}$ for all prime ideals $\mathfrak{p}$ and $\alpha \in \mathbb{Z}_{>0}$, since Eisenstein series are eigenforms and the Hecke algebra is generated by $T\left(\mathfrak{p}^{\alpha}\right)$ for all prime ideals $\mathfrak{p}$ and for all positive integers $\alpha$. Once we see that $E_{k}\left(\chi_{1}, \chi_{2}\right)(z, g)$ is the same as the Eisenstein series in Proposition 3.3.3, we obtain (4.22) by Proposition 3.3.3. Therefore, we will only deal with the case $\mathfrak{m}=\mathfrak{p}^{\alpha}$ for all prime ideals $\mathfrak{p}$ and for all positive integers $\alpha$.

We first observe that for $\mathfrak{p} \mid \mathfrak{n}_{1} \mathfrak{n}_{2}$, we have

$$
\operatorname{val}_{v}(\beta)= \begin{cases}\alpha & \text { if } v=\mathfrak{p} \\ \operatorname{val}_{v}\left(t_{\lambda} \delta\right) & \text { if } v \nmid \mathfrak{n}_{1} \mathfrak{n}_{2} \\ 0 & \text { otherwise }\end{cases}
$$

because $\mathfrak{p}^{\alpha}=\left(t_{\lambda} \delta\right)^{-1} \beta \mathcal{O}_{F}$ and $t_{\lambda} \delta$ is prime $\mathfrak{n}_{1} \mathfrak{n}_{2}$.

If $\mathfrak{p} \mid \mathfrak{n}_{1}$ and $\mathfrak{p} \mid \mathfrak{n}_{2}$, then we have $\operatorname{val}_{\mathfrak{p}}(\beta)>0$ by (4.23). It follows form Lemma 4.1 .4 (4) that

$$
\int_{F_{\mathfrak{p}}} f_{\chi, s, v}\left(\left(\begin{array}{cc}
0 & -1 \\
1 & 0
\end{array}\right)\left(\begin{array}{cc}
1 & n \\
0 & 1
\end{array}\right)\right) \psi_{v}(-\beta n) d n=0
$$


and hence, $c_{\beta}\left(E_{k}\left(\chi_{1}, \chi_{2}\right)\left(z, x_{\lambda}^{-1}\right)\right)=0$ by $(4.20)$. This proves the assertion since $\chi_{1}(\mathfrak{p})=\chi_{2}(\mathfrak{p})=0$ in this case.

If $\mathfrak{p} \nmid \mathfrak{n}_{1}$ and $\mathfrak{p} \mid \mathfrak{n}_{2}$, By Lemma 4.1.4 and (4.23), we have

$$
\begin{aligned}
& \prod_{v<\infty} \int_{F_{v}} f_{\chi, s, v}\left(\left(\begin{array}{cc}
0 & -1 \\
1 & 0
\end{array}\right)\left(\begin{array}{cc}
1 & n_{v} \\
0 & 1
\end{array}\right) x_{\lambda}^{-1}\right) \psi_{v}\left(-\beta n_{v}\right) d n_{v} \\
= & \prod_{v\left|\mathfrak{n}_{1}, v\right| \mathfrak{n}_{2}} \chi_{2, v}^{-1}(-\beta) \varepsilon_{v}\left(2 s+1, \chi_{2, v}^{-1} \psi_{v}\right) \times \prod_{v \nmid \mathfrak{n}_{1}, v \mid \mathfrak{n}_{2}} \chi_{1, v} \chi_{2, v}^{-1}(-\beta)|\beta|_{v}^{2 s} \varepsilon_{v}\left(2 s+1, \chi_{2, v}^{-1}, \psi_{v}\right) \times \prod_{v \mid \mathfrak{n}_{1}, v \nmid \mathfrak{n}_{2}} 1 \times \\
& \prod_{v \nmid \mathfrak{n}_{1} \mathfrak{n}_{2}} \chi_{2, v}^{-1}\left(t_{\lambda} \delta\right)\left|t_{\lambda} \delta\right|_{v}^{s-\frac{1}{2}}\left(1-\chi_{1, v}^{-1}\left(\varpi_{v}\right) q_{v}^{-(2 s+1)}\right) \\
= & L^{\mathfrak{n}}\left(2 s+1, \chi_{1} \chi_{2}^{-1}\right) \times \prod_{v \mid \mathfrak{n}_{2}} \varepsilon_{v}\left(2 s+1, \chi_{2, v}^{-1}, \psi_{v}\right) \times \prod_{v\left|\mathfrak{n}_{1}, v\right| \mathfrak{n}_{2}} \chi_{2, v}^{-1}(-\beta) \times \prod_{v \nmid \mathfrak{n}, v \mid \mathfrak{n}_{2}} \chi_{1, v} \chi_{2, v}^{-1}(-\beta)|\beta|_{v}^{2 s} \times \\
& \prod_{v \nmid \mathfrak{n}_{1} \mathfrak{n}_{2}} \chi_{2, v}^{-1}\left(t_{\lambda} \delta\right)\left|t_{\lambda} \delta\right|_{v}^{s-\frac{1}{2}}
\end{aligned}
$$

which by (4.20), implies that we have

$$
\begin{aligned}
c_{\beta}\left(E_{k}\left(\chi_{1}, \chi_{2}\right)\left(g_{z}, x_{\lambda}^{-1}\right)\right) & =\left[\prod_{v \mid \mathfrak{n}_{2}} \chi_{2, v}^{-1}(-\beta) \times \prod_{v=\mathfrak{p}} \chi_{1, v}(-\beta)|\beta|_{v}^{2 s} \times \prod_{v \nmid \mathfrak{n}_{1} \mathfrak{n}_{2}}\left|t_{\lambda} \delta\right|_{v}^{s-\frac{1}{2}} \chi_{2, v}^{-1}\left(t_{\lambda} \delta\right)\right]_{s=\frac{1-k}{2}} \\
& =\prod_{v \nmid \mathfrak{n}_{1} \mathfrak{n}_{2}} \chi_{2, v}^{-1}\left(-\beta^{-1} t_{\lambda} \delta\right) \times N\left(t_{\lambda} \mathfrak{D}\right)^{k / 2} \chi_{1}\left(\mathfrak{p}^{\alpha}\right) N\left(\mathfrak{p}^{\alpha}\right)^{k-1} \\
& =N\left(t_{\lambda} \mathfrak{D}\right)^{k / 2} \chi_{1}\left(\mathfrak{p}^{\alpha}\right) N\left(\mathfrak{p}^{\alpha}\right)^{k-1} .
\end{aligned}
$$

Note that the second equality is obtained by the product formula for Hecke characters that $\prod_{v} \chi_{2, v}(-\beta)=1$ and the assumption that $\mathfrak{p} \nmid \mathfrak{n}_{1}$. 
If $\mathfrak{p} \mid \mathfrak{n}_{1}$ and $\mathfrak{p} \nmid \mathfrak{n}_{2}$, then by Lemma 4.1.4 and (4.23), we have

$$
\begin{aligned}
& \prod_{v<\infty} \int_{F_{v}} f_{\chi, s, v}\left(\left(\begin{array}{cc}
0 & -1 \\
1 & 0
\end{array}\right)\left(\begin{array}{cc}
1 & n_{v} \\
0 & 1
\end{array}\right) x_{\lambda}^{-1}\right) \psi_{v}\left(-\beta n_{v}\right) d n_{v} \\
= & L^{\mathfrak{n}}\left(2 s+1, \chi_{1} \chi_{2}^{-1}\right) \times \prod_{v \mid \mathfrak{n}_{2}} \varepsilon_{v}\left(2 s+1, \chi_{2, v}^{-1}, \psi_{v}\right) \times \prod_{v\left|\mathfrak{n}_{1}, v\right| \mathfrak{n}_{2}} \chi_{2, v}^{-1}(-\beta) \times \prod_{v \nmid \mathfrak{n}_{1}, v \mid \mathfrak{n}_{2}} \chi_{1, v} \chi_{2, v}^{-1}(-\beta) \times \\
& \prod_{v \mid \mathfrak{n}_{1}, v \nmid \mathfrak{n}_{2}} 1 \times \prod_{v \nmid \mathfrak{n}_{1} \mathfrak{n}_{2}} \chi_{2, v}^{-1}\left(t_{\lambda} \delta\right)\left|t_{\lambda} \delta\right|_{v}^{s-\frac{1}{2}} \\
= & L^{\mathfrak{n}}\left(2 s+1, \chi_{1} \chi_{2}^{-1}\right) \times \prod_{v \mid \mathfrak{n}_{2}} \varepsilon_{v}\left(2 s+1, \chi_{2, v}^{-1}, \psi_{v}\right) \times \prod_{v \mid \mathfrak{n}_{2}} \chi_{2, v}^{-1}(-\beta) \prod_{v \nmid \mathfrak{n}_{1} \mathfrak{n}_{2}} \chi_{2, v}^{-1}\left(t_{\lambda} \delta\right)\left|t_{\lambda} \delta\right|_{v}^{s-\frac{1}{2}} \\
= & L^{\mathfrak{n}}\left(2 s+1, \chi_{1} \chi_{2}^{-1}\right) \times \prod_{v \mid \mathfrak{n}_{2}} \varepsilon_{v}\left(2 s+1, \chi_{2, v}^{-1}, \psi_{v}\right) \times \prod_{v \nmid \mathfrak{n}_{2}} \chi_{2, v}^{-1}\left(-t_{\lambda} \delta \beta^{-1}\right)\left|t_{\lambda} \delta\right|_{v}^{s-\frac{1}{2}} .
\end{aligned}
$$

By (4.20), we obtain

$$
c_{\beta}\left(E_{k}\left(\chi_{1}, \chi_{2}\right)\left(g_{z}, x_{\lambda}^{-1}\right)=N\left(t_{\lambda} \mathfrak{D}\right)^{k / 2} \chi_{2}\left(\mathfrak{p}^{\alpha}\right)\right.
$$

Finally, we assume that $\mathfrak{p} \nmid \mathfrak{n}_{1} \mathfrak{n}_{2}$. It follows from $\mathfrak{p}^{\alpha}=\left(t_{\lambda} \delta\right)^{-1} \beta \mathcal{O}_{F}$ that we have

$$
\operatorname{val}_{v}(\beta)= \begin{cases}\alpha+\operatorname{val}_{v}\left(t_{\lambda} \delta\right) & \text { if } v=\mathfrak{p} \\ \operatorname{val}_{v}\left(t_{\lambda} \delta\right) & \text { if } v \nmid \mathfrak{n}_{1} \mathfrak{n}_{2} \text { and } v \neq \mathfrak{p} \\ 0 & \text { otherwise. }\end{cases}
$$

By Lemma 4.1.4 and (4.24), we have

$$
\begin{aligned}
& \prod_{v<\infty} \int_{F_{v}} f_{\chi, s, v}\left(\left(\begin{array}{cc}
0 & -1 \\
1 & 0
\end{array}\right)\left(\begin{array}{cc}
1 & n_{v} \\
0 & 1
\end{array}\right) x_{\lambda}^{-1}\right) \psi_{v}\left(-\beta n_{v}\right) d n_{v} \\
= & L^{\mathfrak{n}}\left(2 s+1, \chi_{1} \chi_{2}^{-1}\right) \times \prod_{v \mid \mathfrak{n}_{2}} \varepsilon_{v}\left(2 s+1, \chi_{2, v}^{-1}, \psi_{v}\right) \times \prod_{v\left|\mathfrak{n}_{1}, v\right| \mathfrak{n}_{2}} \chi_{2, v}^{-1}(-\beta) \\
& \prod_{v \nmid \mathfrak{n}_{1}, v \mid \mathfrak{n}_{2}} \chi_{1, v} \chi_{2, v}^{-1}(-\beta) \times \prod_{v \mathfrak{n} \mathfrak{n}_{2}} \chi_{2, v}^{-1}\left(t_{\lambda} \delta\right)\left|t_{\lambda} \delta\right|_{v}^{s-\frac{1}{2}} \sum_{n=0}^{\operatorname{val}_{v}\left(\beta t_{\lambda} \delta\right)} \chi_{1, v} \chi_{2, v}^{-1}\left(\varpi_{v}^{n}\right) q_{v}^{-2 s n} \\
= & L^{\mathfrak{n}}\left(2 s+1, \chi_{1} \chi_{2}^{-1}\right) \times \prod_{v \mid \mathfrak{n}_{2}} \varepsilon_{v}\left(2 s+1, \chi_{2, v}^{-1}, \psi_{v}\right) \times \prod_{v \nmid \mathfrak{n}_{1} \mathfrak{n}_{2}} \chi_{2, v}^{-1}\left(-\beta^{-1} t_{\lambda} \delta\right)\left|t_{\lambda} \delta\right|^{s-\frac{1}{2}} \sum_{n=0}^{\operatorname{val}_{v}\left(\beta\left(t_{\lambda} \delta\right)^{-1}\right)} \chi_{1, v} \chi_{2, v}^{-1}\left(\varpi_{v}^{n}\right) q_{v}^{-2 s n} .
\end{aligned}
$$


By (4.20), we have

$$
c_{\beta}\left(E_{k}\left(\chi_{1}, \chi_{2}\right)\left(g_{z}, x_{\lambda}^{-1}\right)\right)=N\left(t_{\lambda} \delta\right)^{k / 2} \sum_{i=0}^{\alpha} \chi_{1}\left(\mathfrak{p}^{i}\right) \chi_{2}\left(\mathfrak{p}^{\alpha-i}\right) N\left(\mathfrak{p}^{i}\right)^{k-1}
$$

This completes the proof.

Recall that we denote by $\chi_{i, f}$ the finite part of $\chi_{i}$ for $i=1,2$. We will write $\mathfrak{n}_{i}=\mathfrak{n}_{i}^{\prime} \times \mathfrak{m}_{i}$ with $\mathfrak{n}_{i}^{\prime}=\prod_{\mathfrak{p \nmid g c d}\left(\mathfrak{n}_{1}, \mathfrak{n}_{2}\right)} \mathfrak{p}^{e_{\mathfrak{p}, i}}$ and $\mathfrak{m}_{i}=\prod_{\mathfrak{p} \mid \operatorname{gcd}\left(\mathfrak{n}_{1}, \mathfrak{n}_{2}\right)} \mathfrak{p}^{e_{\mathfrak{p}, i}}$ for $i=1,2$. We will compute the constant term $c_{0}\left(E_{k}\left(\chi_{1}, \chi_{2}\right)\left(g_{z}, x_{\lambda}^{-1} g\right)\right)$ at the cusp $x_{\lambda}^{-1} g$ for some $g=\left(g_{v}\right)=\left(\left(\begin{array}{ll}a_{v} & b_{v} \\ c_{v} & d_{v}\end{array}\right)\right)_{v} \in \mathrm{GL}_{2}\left(\widehat{\mathcal{O}}_{F}\right)$. By right multiplying some element in $K_{1}(\mathfrak{n})$, we may assume $\operatorname{det} g=1$. Moreover, since $K_{1, v}(\mathfrak{n})=\mathrm{GL}_{2}\left(\mathcal{O}_{v}\right)$ for $v \nmid \mathfrak{n}_{1} \mathfrak{n}_{2}$, we may assume further that $g_{v}$ is the identity matrix for $v \nmid \mathfrak{n}_{1} \mathfrak{n}_{2}$.

Proposition 4.3.2. Let the notation and the assumption be as above. We set $c=\left(c_{v}\right)_{v}$ and $d=\left(d_{v}\right)_{v}$. Also, we set $c_{\mathfrak{n}_{2}}\left(\right.$ resp. $\left.d_{\mathfrak{n}_{1}}\right)$ be the $\mathfrak{n}_{2}$-part of c (resp. $\mathfrak{n}_{1}$-part of d). Assume further that the following conditions hold

1. $\operatorname{val}_{v}\left(c_{v}\right) \geq e_{\mathfrak{p}, 1}$ for all $v \mid \mathfrak{n}_{1}^{\prime}$,

2. $\operatorname{val}_{v}\left(c_{v}\right)=0$ for all $v \mid \mathfrak{n}_{2}^{\prime}$,

3. $\operatorname{val}_{v}\left(c_{v}\right)=e_{\mathfrak{p}, 1}$ for all $v \mid \operatorname{gcd}\left(\mathfrak{n}_{1}, \mathfrak{n}_{2}\right)$.

Then the constant term $c_{0}\left(E_{k}\left(\chi_{1}, \chi_{2}\right)\left(g_{z}, x_{\lambda}^{-1} g\right)\right)$ is

$$
\begin{aligned}
& \frac{1}{2^{d}} L^{\mathfrak{n}}\left(1-k, \chi_{1} \chi_{2}^{-1}\right) \chi_{1}^{-1}\left(\mathfrak{n}_{2}^{\prime}\right) N\left(\mathfrak{n}_{2}^{\prime}\right)^{-1} \times \prod_{v \mid \operatorname{gcd}\left(\mathfrak{n}_{1}, \mathfrak{n}_{2}\right)} M_{v, \frac{1-k}{2}, e_{\mathfrak{p}, 1}} \times \prod_{v \mid \mathfrak{n}_{2}} \varepsilon_{v}\left(2-k, \chi_{2, v}^{-1}, \psi_{v}\right)^{-1} \times \\
& \chi_{2}^{-1}\left(t_{\lambda} \mathfrak{D}\right) N\left(t_{\lambda} \mathfrak{D}\right)^{k / 2} \chi_{1, f}^{-1}\left(d_{\mathfrak{n}_{1}}\right) \chi_{2, f}\left(-c_{\mathfrak{n}_{2}} \mathfrak{m}_{1}^{-1}\right)
\end{aligned}
$$

where $M_{v, s, e_{\mathfrak{p}, 1}}$ was defined in Lemma 4.1.5. Here $\chi_{1, f}\left(d_{\mathfrak{n}_{1}}\right)$ and $\chi_{2, f}\left(c_{\mathfrak{n}_{2}} \mathfrak{m}_{1}^{-1}\right)$ are respectively defined by the isomorphisms $\prod_{v \mid \mathfrak{n}_{i}} \mathcal{O}_{v}^{\times} / 1+\varpi^{e_{\mathfrak{p}, i}} \mathcal{O}_{v} \simeq\left(\mathcal{O} / \mathfrak{n}_{i} \mathcal{O}\right)^{\times}$for $i=1,2$.

Otherwise, if any one of the above conditions does not hold, then the constant term is 0. 
ProOF. By the same computation as in Proposition 4.3.1, we know that $c_{0}\left(E_{k}\left(\chi_{1}, \chi_{2}\right)\left(g_{z}, x_{\lambda}^{-1} g\right)\right)$ equals

$$
\left[\frac{C_{\infty}(k)^{-d} L^{\mathfrak{n}}\left(2-k, \chi_{1}, \chi_{2}^{-1}\right)}{\prod_{v \mid \mathfrak{n}_{2}} \varepsilon_{v}\left(2-k, \chi_{2, v}^{-1}, \psi_{v}\right)} \int_{\mathbb{A}_{F}} f_{\chi, s}\left(\left(\begin{array}{cc}
0 & -1 \\
1 & 0
\end{array}\right)\left(\begin{array}{cc}
1 & n \\
0 & 1
\end{array}\right) g_{z} x_{\lambda}^{-1} g\right) d n\right]_{s=\frac{1-k}{2}} .
$$

It follows from Lemma 4.1.5 that if there exists a finite place $v \mid \mathfrak{n}_{1}$ satisfying $\operatorname{val}_{v}\left(c_{v}\right)<e_{\mathfrak{p}, 1}$ or $v \mid \mathfrak{n}_{2}$ satisfying $0<\operatorname{val}_{v}\left(c_{v}\right)<e_{\mathfrak{p}, 2}$, then the integral $\int_{F_{v}} f_{\chi, s, v}\left(\left(\begin{array}{cc}0 & -1 \\ 1 & 0\end{array}\right)\left(\begin{array}{cc}1 & n_{v} \\ 0 & 1\end{array}\right) \gamma\right) d n_{v}$ is 0 .

Now we assume that $\operatorname{val}_{v}\left(c_{v}\right)>e_{\mathfrak{p}, 1}$ for all $v \mid \mathfrak{n}_{1}$ and assume that $\operatorname{val}_{v}\left(c_{v}\right)=0$ for all $v \mid \mathfrak{n}_{2}$. For infinite places, we have

$$
\left[\prod_{v \mid \infty} \int_{F_{v}} f_{\chi, s, v}\left(\left(\begin{array}{cc}
0 & -1 \\
1 & 0
\end{array}\right)\left(\begin{array}{cc}
1 & n_{v} \\
0 & 1
\end{array}\right) g_{z}\right) d n_{v}\right]_{s=\frac{1-k}{2}}=\frac{C_{\infty}(k)^{d}}{2^{d}}
$$

For finite places $v \nmid \mathfrak{n}_{1} \mathfrak{n}_{2}$, we have

$$
\left[\prod_{v \nmid \mathfrak{n}_{1} \mathfrak{n}_{2}} \int_{F_{v}} f_{\chi, s, v}\left(\left(\begin{array}{cc}
0 & -1 \\
1 & 0
\end{array}\right)\left(\begin{array}{cc}
1 & n_{v} \\
0 & 1
\end{array}\right) x_{\lambda}^{-1} g_{v}\right) d n_{v}\right]_{s=\frac{1-k}{2}}=N\left(t_{\lambda} \mathfrak{D}\right)^{k / 2} \chi_{2}^{-1}\left(t_{\lambda} \mathfrak{D}\right) \frac{L^{\mathfrak{n}}\left(1-k, \chi_{1} \chi_{2}^{-1}\right)}{L^{\mathfrak{n}}\left(2-k, \chi_{1} \chi_{2}^{-1}\right)}
$$

For finite places $v \mid \mathfrak{n}_{1}^{\prime}$, we have

$$
\left[\prod_{v \mid \mathfrak{n}_{1}^{\prime}} \int_{F_{v}} f_{\chi, s, v}\left(\left(\begin{array}{cc}
0 & -1 \\
1 & 0
\end{array}\right)\left(\begin{array}{cc}
1 & n_{v} \\
0 & 1
\end{array}\right) g_{v}\right) d n_{v}\right]_{s=\frac{1-k}{2}}=\prod_{v \mid \mathfrak{n}_{1}^{\prime}} \chi_{1, v} \chi_{2, v}\left(d_{v}\right)=\prod_{v \mid \mathfrak{n}_{1}^{\prime}} \chi_{1, v}\left(d_{v}\right) .
$$

For finite places $v \mid \mathfrak{n}_{2}^{\prime}$, we have

$$
\left[\prod_{v \mid \mathfrak{n}_{2}^{\prime}} \int_{F_{v}} f_{\chi, s, v}\left(\left(\begin{array}{cc}
0 & -1 \\
1 & 0
\end{array}\right)\left(\begin{array}{cc}
1 & n \\
0 & 1
\end{array}\right) g_{v}\right) d n_{v}\right]_{s=\frac{1-k}{2}}=\prod_{v \mid \mathfrak{n}_{2}^{\prime}} \chi_{2, v}^{-1}\left(-c_{v}\right) \chi_{1, v}\left(\varpi^{-e_{\mathfrak{p}, 2}}\right) q_{v}^{-e_{\mathfrak{p}, 2}}
$$

which equals $\chi_{1}\left(\mathfrak{n}_{2}^{\prime}\right)^{-1} N\left(\mathfrak{n}_{2}^{\prime}\right)^{-1} \prod_{v \mid \mathfrak{n}_{2}^{\prime}} \chi_{2, v}^{-1}\left(-c_{v}\right)$. For finite places $v \mid \operatorname{gcd}\left(\mathfrak{n}_{1}, \mathfrak{n}_{2}\right)$, since $\operatorname{val}_{c}\left(c_{v}\right)=e_{\mathfrak{p}, 1}$, by Lemma 2.2.3, one can decompose $g_{v}$ as

$$
g_{v}=\left(\begin{array}{ll}
a_{v} & b_{v} \\
c_{v} & d_{v}
\end{array}\right)=\left(\begin{array}{cc}
c_{v}^{-1} \varpi^{e_{\mathfrak{p}, 1}} & b_{v} \\
0 & d_{v}
\end{array}\right)\left(\begin{array}{cc}
1 & 0 \\
\varpi^{e_{\mathfrak{p}, 1}} & 1
\end{array}\right) \kappa
$$

for some $\kappa \in K_{1, v}(\mathfrak{n})$. Then

$$
\begin{aligned}
& \prod_{v \mid \operatorname{gcd}\left(\mathfrak{n}_{1}, \mathfrak{n}_{2}\right)} \int_{F_{\mathfrak{p}}} f_{\chi, s, v}\left(\left(\begin{array}{cc}
0 & -1 \\
1 & 0
\end{array}\right)\left(\begin{array}{cc}
1 & n_{v} \\
0 & 1
\end{array}\right) g_{v}\right) d n_{v} \\
= & \prod_{v \mid \operatorname{gcd}\left(\mathfrak{n}_{1}, \mathfrak{n}_{2}\right)} \chi_{1, v}\left(d_{v}\right) \chi_{2, v}\left(c_{v}^{-1} \varpi^{e_{\mathfrak{p}, 1}}\right) \int_{F_{\mathfrak{p}}} f_{\chi, s, v}\left(\left(\begin{array}{cc}
0 & -1 \\
1 & 0
\end{array}\right)\left(\begin{array}{cc}
1 & n_{v} \\
0 & 1
\end{array}\right)\left(\begin{array}{cc}
1 & 0 \\
\varpi^{e_{\mathfrak{p}, 1}} & 1
\end{array}\right)\right) d n_{v},
\end{aligned}
$$


which equals $\prod_{v \mid \operatorname{gcd}\left(\mathfrak{n}_{1}, \mathfrak{n}_{2}\right)} \chi_{1, v}\left(d_{v}\right) \chi_{2, v}\left(c_{v}^{-1} \varpi^{e_{\mathfrak{p}, 1}}\right) M_{v, s, e_{\mathfrak{p}, 1}}$ by Lemma 4.1.5. Finally, to finish the proof, we note that $\prod_{v \mid \mathfrak{n}_{1}} \chi_{1, v}\left(d_{v}\right)=\chi_{1, f}^{-1}\left(d_{\mathfrak{n}_{1}}\right)$ and $\prod_{v \mid \mathfrak{n}_{2}} \chi_{2, f}\left(-c_{v}^{-1} \varpi^{e_{\mathfrak{p}, 2}}\right)=\chi_{2, f}\left(-c_{\mathfrak{n}_{2}} \mathfrak{m}_{1}^{-2}\right)$.

\subsection{More on the constant terms of Eisenstein series}

In the previous section, we constructed Eisenstein series adelically and computed their constant terms at different cusps. Indeed, this can be done in the classical setting. When $F=\mathbb{Q}$, this is well-known (see [Ohta3] for example). When $F \neq \mathbb{Q}$, this has been done by T. Ozawa [Oz]. In this section, we prove that Proposition 4.3.2 coincides with the result obtained from the classical approach when $F=\mathbb{Q}$. When $F \neq \mathbb{Q}$, it will be addressed in Remark 4.4.2.

In the remainder of this section, we assume that $F=\mathbb{Q}$. Let the notation be as in the previous section. We now recall the result of [Ohta3]. Let $\chi_{1}$ and $\chi_{2}$ be primitive Dirichlet characters of conductor $N_{1}$ and $N_{2}$, respectively. For simplicity, we will write $N=N_{1} N_{2}$. Recall that for $i=1,2$, we decompose $N_{i}$ as $N_{i}=\prod_{p \mid N_{i}} p^{e_{p, i}}$ for some $e_{p, i} \in \mathbb{Z}_{>0}$. Also, we decompose $\chi_{i}$ as $\chi_{i}=\prod_{p \mid N_{i}} \chi_{i}^{(p)}$, where $\chi_{i}^{(p)}$ is the restriction of $\chi_{i}$ to $\left(\mathbb{Z} / p^{e_{p, i}} \mathbb{Z}\right)^{\times}$.

Assume that $\chi_{1}$ is a nontrivial character. For $k \geq 2$, the constant term of $E_{k}\left(\chi_{1}, \chi_{2}\right)$ at the cusp $g=\left(\begin{array}{ll}a & b \\ c & d\end{array}\right) \in \mathrm{SL}_{2}(\mathbb{Z})$ is 0 if $N_{1}$ does not divide $c$. If $N_{1} \mid c$, then it is given by

$$
\frac{1}{2} \frac{\tau\left(\chi_{1}^{-1} \chi_{2}\right)}{\tau\left(\chi_{1}^{-1}\right)}\left(\frac{\operatorname{cond}\left(\chi_{1}\right)}{\operatorname{cond}\left(\chi_{1}^{-1} \chi_{2}\right)}\right)^{k} \chi_{2}\left(\frac{-c}{N_{1}}\right) \chi_{1}^{-1}(a) L^{N}\left(1-k, \chi_{1} \chi_{2}^{-1}\right) .
$$

On the adelic side, by Definition 3.2.1, we have to find the constant term of $E_{k}\left(\chi_{1}, \chi_{2}\right)$ at the cusp $g=\left(\begin{array}{ll}a & b \\ c & d\end{array}\right)^{-1}=\left(\begin{array}{cc}d & -b \\ -c & a\end{array}\right) \in \mathrm{SL}_{2}\left(\mathbb{A}_{\mathbb{Q}, f}\right)$. It is easy to see that if one of the conditions in Proposition 4.3.2 does not hold, then the results obtained on both classical side and adelic side are all 0 . Now we assume that $g$ satisfies the conditions in Proposition 4.3.2. Then one can rewrite the result of 
Proposition 4.3.2 as

$$
\frac{1}{2} L^{N}\left(1-k, \chi_{1} \chi_{2}^{-1}\right) N_{2}^{\prime-1} \chi_{1}^{-1}\left(N_{2}^{\prime}\right) \chi_{2}\left(N_{1}^{\prime}\right) \times \prod_{v \mid \operatorname{gcd}\left(\mathfrak{n}_{1}, \mathfrak{n}_{2}\right)} M_{v, \frac{1-k}{2}, e_{\mathfrak{p}, 1}} \times \prod_{v \mid \mathfrak{n}_{2}} \varepsilon_{v}\left(2-k, \chi_{2, v}^{-1}, \psi_{v}\right)^{-1} \times \chi_{1}^{-1}(d) \chi_{2}\left(-c N_{1}^{-1}\right)
$$

Therefore, the main idea to see Proposition 4.3.2 coincides with (4.25) is to show

$$
N_{2}^{\prime-1} \chi_{1}^{-1}\left(N_{2}^{\prime}\right) \chi_{2}\left(N_{1}^{\prime}\right) \times \prod_{v \mid \operatorname{gcd}\left(\mathfrak{n}_{1}, \mathfrak{n}_{2}\right)} M_{v, \frac{1-k}{2}, e_{\mathfrak{p}, 1}} \times \prod_{v \mid \mathfrak{n}_{2}} \varepsilon_{v}\left(2-k, \chi_{2, v}^{-1}, \psi_{v}\right)^{-1}=\frac{\tau\left(\chi_{1}^{-1} \chi_{2}\right)}{\tau\left(\chi_{1}^{-1}\right)}\left(\frac{\operatorname{cond}\left(\chi_{1}\right)}{\operatorname{cond}\left(\chi_{1}^{-1} \chi_{2}\right)}\right)^{k}
$$

To simplify our computation, we will only deal with two cases: (1) $N_{1}$ and $N_{2}$ are coprime, (2) $N_{1}$ and $N_{2}$ are prime powers whose prime divisors are the same. The argument for general cases is more complicated but based on those two cases.

To simplify the result on adelic side, we first prove the following lemma.

Lemma 4.4.1. Let the notation be as above. Then we have

$$
\prod_{p \mid N_{2}} \varepsilon_{p}\left(2-k, \chi_{2, p}, \psi_{p}\right)=N_{2}^{k-1} \tau\left(\chi_{2}\right)^{-1}
$$

Proof. By Lemma 2.5.2, we have

$$
\begin{aligned}
\prod_{p \mid N_{2}} \varepsilon_{p}\left(2-k, \chi_{2, p}, \psi_{p}\right) & =\prod_{p \mid N_{2}} p^{-(2-k) e_{p, 2}} \chi_{2, p}\left(p^{-e_{p, 2}}\right) \tau\left(\chi_{2, v}\right) \\
& =N_{2}^{k-2} \prod_{p \mid N_{2}} \chi_{2, p}\left(p^{-e_{p, 2}} \overline{\tau\left(\chi_{2}^{(p)}\right)}\right. \\
& =N_{2}^{k-2} \prod_{p \mid N_{2}} \chi_{2}^{(p)}\left(\frac{N_{2}}{p^{e_{p}, 2}}\right)^{-1} p^{e_{p, 2}} \tau\left(\chi_{2}^{(p)}\right)^{-1} \\
& =N_{2}^{k-1} \tau\left(\chi_{2}\right)^{-1} .
\end{aligned}
$$

Here the last equality is obtained by the property of Gauss sums that if $\theta_{1}$ and $\theta_{2}$ are primitive Dirichlet characters whose conductors are coprime, then we have

$$
\tau\left(\theta_{1} \theta_{2}\right)=\tau\left(\theta_{1}\right) \tau\left(\theta_{2}\right) \theta_{1}\left(\operatorname{cond}\left(\theta_{2}\right)\right) \theta_{2}\left(\operatorname{cond}\left(\theta_{1}\right)\right)
$$

The proof is complete. 
Case 1: Assume that $N_{1}$ and $N_{2}$ are coprime. one can simplify (4.25) as

$$
\frac{1}{2} \tau\left(\chi_{2}\right) N_{2}^{-k} \chi_{2}(-c) \chi_{1}^{-1}\left(a N_{2}\right) L\left(1-k, \chi_{1} \chi_{2}^{-1}\right)
$$

On the other hand, one can simplify Proposition 4.3.2 as

$$
\frac{1}{2} N_{2}^{-1} \chi_{1}^{-1}\left(a N_{2}\right) \chi_{2}(-c) L\left(1-k, \chi_{1} \chi_{2}^{-1}\right) \prod_{p \mid N_{2}} \varepsilon_{p}\left(2-k, \chi_{2, p}^{-1} \psi_{p}\right)^{-1}
$$

By Lemma 4.4.1, we see that (4.26) and (4.27) are equal.

Case 2: We assume that $N_{1}=p^{e_{1}}$ and $N_{2}=p^{e_{2}}$ for some $e_{1}, e_{2}>0$. Since the computation for the case $e_{1}>e_{2}$ is essentially the same as the computations for the cases $e_{1}<e_{2}$ and $e_{1}=e_{2}$, we will only work on the case $e_{1}>e_{2}$. In this case, one can simplify (4.25) as

$$
\frac{1}{2} \frac{\tau\left(\chi_{1}^{-1} \chi_{2}\right)}{\tau\left(\chi_{1}^{-1}\right)} \chi_{2}\left(\frac{-c}{p^{e_{1}}}\right) \chi_{1}^{-1}(a) L\left(1-k, \chi_{1} \chi_{2}^{-1}\right)
$$

On the adelic side, one can rewrite the result of Proposition 4.3.2 as

$$
\frac{1}{2} L\left(1-k, \chi_{1} \chi_{2}^{-1}\right) \chi_{1, p}(a) \chi_{2, p}\left(c^{-1} p^{e_{1}}\right) \varepsilon_{p}\left(2-k, \chi_{2, p}^{-1}, \psi_{p}\right)^{-1} M_{p, e_{1}}
$$

By Lemma 4.4.1 and Lemma 2.5.2, we again see that the result on the adelic side coincides with (4.25).

Remark 4.4.2. When $F$ is a totally real field with $d=[F: \mathbb{Q}]$, Ozawa's result is as follows. Let $g \in \Gamma_{0}\left(t_{\lambda} \mathfrak{D}, \mathcal{O}_{F}\right) \cap \mathrm{SL}_{2}(F)$, then the constant term of $E_{k}\left(\chi_{1}, \chi_{2}\right)$ at the cusp $g$ is

$$
N\left(t_{\lambda} \mathfrak{D}\right)^{-k / 2} \frac{1}{2^{d}} \frac{\tau\left(\chi_{1}^{-1} \chi_{2}\right)}{\tau\left(\chi_{1}^{-1}\right)}\left(\frac{N\left(\mathfrak{n}_{1}\right.}{N(\mathfrak{c})}\right)^{k} \operatorname{sgn}(-c)^{r} \chi_{2}\left(c\left(\mathfrak{n}_{1} \mathfrak{D} t_{\lambda}\right)^{-1}\right) \operatorname{sgn}(a)^{q} \chi_{1}^{-1}(a) L^{\mathfrak{n}}\left(1-k, \chi_{1} \chi_{2}^{-1}\right) .
$$

if $c \in \mathfrak{n}_{1} t_{\lambda} \mathfrak{D}$. On the adelic side, by Definition 3.2.1, we have to find the constant term of $E_{k}\left(\chi_{1}, \chi_{2}\right)$ at the cusp $x_{\lambda}^{-1} g^{-1} \in x_{\lambda}^{-1} \mathrm{SL}_{2}\left(\mathbb{A}_{\mathbb{Q}, f}\right)$. Then one can rewrite the result of Proposition 4.3 .2 as

$$
\begin{aligned}
& \frac{1}{2^{d}} L^{\mathfrak{n}}\left(1-k, \chi_{1} \chi_{2}^{-1}\right) \chi_{1}^{-1}\left(\mathfrak{n}_{2}^{\prime}\right) N\left(\mathfrak{n}_{2}^{\prime}\right)^{-1} \chi_{2}\left(\mathfrak{n}_{1}^{\prime}\right) \times \prod_{v \mid \operatorname{gcd}\left(\mathfrak{n}_{1}, \mathfrak{n}_{2}\right)} M_{v, \frac{1-k}{2}, e_{\mathfrak{p}, 1}} \times \prod_{v \mid \mathfrak{n}_{2}} \varepsilon_{v}\left(2-k, \chi_{2, v}^{-1}, \psi_{v}\right)^{-1} \times \\
& N\left(t_{\lambda} \mathfrak{D}\right)^{-k / 2} \operatorname{sgn}^{q}(a) \chi_{1}^{-1}(a) \operatorname{sgn}^{r}(-c) \chi_{2}\left(c\left(\mathfrak{n}_{1} t_{\lambda} \mathfrak{D}\right)^{-1}\right) .
\end{aligned}
$$


Thus, to check Proposition 4.3.2 coincides with Ozawa's result [Oz], it suffices to check

$$
\chi_{1}^{-1}\left(\mathfrak{n}_{2}^{\prime}\right) N\left(\mathfrak{n}_{2}^{\prime}\right)^{-1} \chi_{2}\left(\mathfrak{n}_{1}^{\prime}\right) \prod_{v \mid \operatorname{gcd}\left(\mathfrak{n}_{1}, \mathfrak{n}_{2}\right)} M_{v, \frac{1-k}{2}, e_{\mathfrak{p}, 1}} \times \prod_{v \mid \mathfrak{n}_{2}} \varepsilon_{v}\left(2-k, \chi_{2, v}^{-1}, \psi_{v}\right)^{-1}=\frac{\tau\left(\chi_{1}^{-1} \chi_{2}\right)}{\tau\left(\chi_{1}^{-1}\right)}\left(\frac{N\left(\mathfrak{n}_{1}\right)}{N(\mathfrak{c})}\right)^{k}
$$

which is similar to the case $F=\mathbb{Q}$ but more complicated. 


\section{CHAPTER 5}

$\Lambda$-adic modular forms

\subsection{Definitions}

Let $p$ be an odd prime, and let $u=(1+p)^{p^{e}} \in \mathbb{Z}_{p}^{\times}$, where $p^{e}=\left[F \cap \mathbb{Q}_{\infty}: \mathbb{Q}\right]$. Let $\gamma$ be a topological generator of $\operatorname{Gal}\left(F_{\infty} / F\right) \simeq \mathbb{Z}_{p}$ such that $\gamma \cdot \zeta=\zeta^{u}$ for all $p$-power roots of unity $\zeta$.

For any integral ideal $\mathfrak{a}$ of $F$ prime to $p$, we have

$$
N(\mathfrak{a})=\delta u^{s(\mathfrak{a})}=\omega(N(\mathfrak{a}))\langle N(\mathfrak{a})\rangle
$$

for some $\delta \in \mu_{p-1}$ and $s(\mathfrak{a}) \in \mathbb{Z}_{p}$. Here $\omega$ is the Teichmüller character and $\langle\cdot\rangle$ is the projection $\mathbb{Z}_{p}^{\times} \rightarrow 1+p \mathbb{Z}_{p}$. Moreover, we have

$$
\omega \otimes\langle\rangle: \mathbb{Z}_{p}^{\times} \simeq(\mathbb{Z} / p \mathbb{Z})^{\times} \times 1+p \mathbb{Z}_{p}
$$

For simplicity, we put $\omega(\mathfrak{a})=\omega(N(\mathfrak{a}))$ for all ideals $\mathfrak{a}$ of $F$ prime to $p$.

For each narrow ray class character $\chi$ modulo $\mathfrak{n}$ or $\mathfrak{n} p$, we associate a homomorphism,

$$
\bar{\chi}:{\underset{r}{r}}_{\lim } I_{\mathfrak{n} p^{r}} \longrightarrow \mathbb{Z}_{p}[\chi][[T]]
$$

as follows. For each ideal $\mathfrak{a}$ prime to $\mathfrak{n} p, \bar{\chi}(\mathfrak{a})$ is defined to be $\chi(\mathfrak{a})(1+T)^{s(\mathfrak{a})}$, where $s(\mathfrak{a}) \in \mathbb{Z}_{p}$ is defined as in (5.1). We extend the character $\chi$ to a character of $\mathrm{Cl}_{F}^{+}$by setting $\chi(\mathfrak{a})=0$, and hence, $\bar{\chi}(\mathfrak{a})=0$ if $\mathfrak{a}$ is not prime to $\mathfrak{n} p$. Recall that $I_{\mathfrak{m}}$ is the set of fractional ideals of $F$ relatively prime to $\mathfrak{m}$.

Let $\mu_{p^{\infty}}$ be the group of all $p$-power roots of unity, and let $\widehat{\mu_{p} \infty}$ be the group of all characters of $\mu_{p^{\infty}}$ with values in $\mathbb{C}$. Let $\mathcal{O}_{\infty} \subset \mathbb{C}_{p}$ be a complete valuation ring containing $\mu_{p^{\infty}}$ and all values of 
narrow ray class characters of modulo $\mathfrak{n} p$. We put $\Lambda=\mathcal{O}_{\infty}[|T|]$. For each integer $k$ and for each $\zeta \in \mu_{p^{\infty}}$, we define two evaluation maps

$$
v_{k, \zeta}, v_{k, \zeta}^{\prime}: \Lambda \longrightarrow \mathcal{O}_{\infty}
$$

by $v_{k, \zeta}(T)=\zeta u^{k-2}-1\left(\operatorname{resp} . v_{k, \zeta}^{\prime}(T)=\zeta u^{k}-1\right)$. Note that for each $\zeta \in \mu_{p^{\infty}}$, there exists $\rho=\rho_{\zeta} \in \widehat{\mu}_{p^{\infty}}$ such that $\rho(u)=\zeta$. We will also write $v_{k, \zeta}\left(\operatorname{resp} . v_{k, \zeta}^{\prime}\right)$ as $v_{k, \rho}\left(\right.$ resp. $\left.v_{k, \rho}^{\prime}\right)$, which was the notation used in Ohta's papers. For simplicity, we will write $\rho(N(\mathfrak{a}))$ as $\rho(\mathfrak{a})$ for all $\mathfrak{a} \in I_{p}$. Let

$$
\mathfrak{X}:=\left\{(k, \zeta) \mid k \geq 2, \zeta^{p^{r}}=1 \text { for some } r \geq 0\right\}
$$

\section{Definition 5.1.1.}

1. Let $\mathfrak{n}$ be an integral ideal of $\mathcal{O}_{F}$. A $\Lambda$-adic modular form $\mathcal{F}$ over $F$ of level $\mathfrak{n} p$ (weight $k$ ) is a set of elements of $\Lambda$

$$
\left\{\begin{array}{cc}
C(\mathfrak{a}, \mathcal{F}) & \text { for all nonzero integral ideals } \mathfrak{a} \text { of } \mathcal{O}_{F} \\
C_{\lambda}(0, \mathcal{F}) & \text { for } \lambda=1, \ldots, h_{F}^{+}
\end{array}\right\}
$$

with the property that for all but finitely many $v_{k, \zeta}$ with $(k, \zeta) \in \mathfrak{X}$ ( $k$ is fixed), there is an adelic modular form $f$ of weight $k$ and level $\mathfrak{n} p^{r}$ such that for each integral ideal $\mathfrak{a}$ of $F$, the normalized $\mathfrak{a}$-th Fourier coefficient satisfies $C(\mathfrak{a}, f)=v_{k, \zeta}(C(\mathfrak{a}, \mathcal{F}))$ and constant terms satisfy $C_{\lambda}(0, f)=v_{k, \zeta}\left(C_{\lambda}(0, \mathcal{F})\right)$

2. A $\Lambda$-adic form is said to be a cusp form if $v(\mathcal{F})$ is a cusp form for almost all specializations.

3. Let $\chi$ be a narrow ray class character modulo $\mathfrak{n} p$. We say that $\mathcal{F}$ is of character $\chi$ if $v_{k, \zeta}(\mathcal{F})$ has character $\chi \omega^{2-k} \rho_{\zeta}$.

We denote by $M(\mathfrak{n}, \chi ; \Lambda)$ and $S(\mathfrak{n}, \chi ; \Lambda)$ the space of $\Lambda$-adic modular forms and the space of $\Lambda$-adic cusp forms of level $\mathfrak{n}$ and character $\chi$, respectively. 
One can define $\Lambda$-adic modular forms and $\Lambda$-adic cusp forms with respect to the specialization $v_{k, \zeta}^{\prime}$ in the same manner. Under this definition, we say that a $\Lambda$-adic modular form $\mathcal{F}$ is of character $\chi$ if $v_{k, \zeta}^{\prime}(\mathcal{F})$ is of character $\chi \omega^{-k} \rho$. We denote by $M^{\prime}(\mathfrak{n}, \chi ; \Lambda)$ and $S^{\prime}(\mathfrak{n}, \chi ; \Lambda)$ the corresponding spaces of $\Lambda$-adic modular forms and $\Lambda$-adic cusp forms, respectively.

The reason we mention different specializations is because different places in the literature, people use different specializations. For example, in [H2] and [H3], Hida used the specialization $v_{k, \zeta}^{\prime}$, while Wiles used the specialization $v_{k, \zeta}$ in [Wil2] and [Wil3]. Indeed, these specializations are equivalent, which we address in the following lemma.

Lemma 5.1.2. We have an isomorphism of $\Lambda$-modules

$$
M^{\prime}\left(\mathfrak{n}, \chi \omega^{2} ; \Lambda\right) \simeq M(\mathfrak{n}, \chi ; \Lambda)
$$

ProOF. The assertion is well-known. Given any $\mathcal{F}^{\prime} \in M^{\prime}(\mathfrak{n}, \chi ; \Lambda)$, we set $\mathcal{F}(T)=\mathcal{F}^{\prime}\left(u^{2}(1+T)-1\right)$. Then we have

$$
v_{k, \rho}(\mathcal{F}(T))=\mathcal{F}\left(\rho(u) u^{k-2}-1\right)=\mathcal{F}^{\prime}\left(\rho(u) u^{k}-1\right)=v_{k, \zeta}^{\prime}\left(\mathcal{F}^{\prime}(T)\right)
$$

which, by the definition of $M^{\prime}(\mathfrak{n}, \chi ; \Lambda)$, is an adelic modular form of level $\mathfrak{n} p^{r}$ and character $\chi \omega^{2-k} \rho$ for almost all $(k, \zeta) \in \mathfrak{X}$. Therefore, $\mathcal{F}(T)$ is in $M(\mathfrak{n}, \chi ; \Lambda)$, and clearly this provides a bijection.

Remark 5.1.3. In this paper, we usually consider the space $M(\mathfrak{n}, \chi ; \Lambda)$. We will only use $M^{\prime}(\mathfrak{n}, \chi ; \Lambda)$ in Chapter 6, where we will prove a control theorem for $M^{\prime}(\mathfrak{n}, \chi ; \Lambda)$ for an arbitrary narrow ray class character $\chi$ modulo $\mathfrak{n} p$ and then deduce a control theorem for $M(\mathfrak{n}, \chi ; \Lambda)$ from Lemma 5.1.2.

Note that the Hecke action on the space of adelic modular forms induces an actions on the space of $\Lambda$-adic modular forms. Indeed, Hecke actions commute with specialization map $v_{k, \zeta}$ and $v_{k, \zeta}^{\prime}$. For details, we refer the reader to [Wil2]. Thus the Hida idempotent element $e$ acts on $M(\mathfrak{n}, \chi ; \Lambda)($ resp. 
$\left.M^{\prime}(\mathfrak{n}, \chi ; \Lambda)\right)$ and preserves the subspace $S(\mathfrak{n}, \chi ; \Lambda)\left(\operatorname{resp} S^{\prime}(\mathfrak{n}, \chi ; \Lambda)\right)$. We define $M^{\text {ord }}(\mathfrak{n}, \chi ; \Lambda)=$ $e \cdot M(\mathfrak{n}, \chi ; \Lambda)$ and define $S^{\text {ord }}(\mathfrak{n}, \chi ; \Lambda), M^{\text {ord }}(\mathfrak{n}, \chi ; \Lambda)$, and $S^{\prime \text { ord }}(\mathfrak{n}, \chi ; \Lambda)$ in the same manner. We denote by $\mathcal{H}^{\text {ord }}(\mathfrak{n}, \chi, \Lambda) \subset \operatorname{End}_{\Lambda}\left(M^{\text {ord }}(\mathfrak{n}, \chi ; \Lambda)\right)\left(\operatorname{resp} . h^{\text {ord }}(\mathfrak{n}, \chi ; \Lambda) \subset \operatorname{End}_{\Lambda}\left(S^{\text {ord }}(\mathfrak{n}, \chi ; \Lambda)\right)\right.$ the Hecke algebra (resp. cuspidal Hecke algebra) generated over $\Lambda$ by Hecke operators $T(\mathfrak{p})$ for all prime ideals $\mathfrak{p}$ and $S(\mathfrak{q})$ for all prime ideals $\mathfrak{q}$ not dividing $\mathfrak{n} p$.

Note that the isomoiphism in Lemma 5.1.2 is Hecke-equivariant since specialization maps commute with Hecke operators and since for each $\mathcal{F} \in M(\mathfrak{n}, \chi ; \Lambda)$, if $\mathcal{F}^{\prime}$ is the image of $\mathcal{F}$ under the isomorphism, we have $v_{k, \zeta}(\mathcal{F})=v_{k, \zeta}^{\prime}\left(\mathcal{F}^{\prime}\right)$ for all $(k, \zeta) \in \mathfrak{X}$.

\section{$5.2 \Lambda$-adic Eisenstein series}

Eisenstein series provide interesting examples of $\Lambda$-adic modular forms. We recall their construction in this subsection.

Let $\chi$ be an narrow ideal class character of conductor $\mathfrak{n}$ or $\mathfrak{n} p$. We assume that $\chi$ is even and is not of type $W$ in the sense of [Wil3], i.e., $F_{\chi}$ is not contained in $F_{\infty}$. Let $L_{p}(s, \chi)$ be the Deligne-Ribet $p$-adic $L$-function (see $[\mathrm{DR}]$ for the definition). It satisfies interpolation property that

$$
L_{p}(1-k, \chi)=L\left(1-k, \chi \omega^{-k}\right) \prod_{\mathfrak{p} \mid p}\left(1-\chi \omega^{-k}(p) N(p)^{k-1}\right) .
$$

for positive integers $k$. Moreover, there exist relatively prime $G_{\chi}(T)$ and $H_{\chi}(T)$ in $\Lambda=\mathbb{Z}_{p}[\chi][|T|]$ such that

$$
L_{p}(1-s, \chi)=G_{\chi}\left(u^{s}-1\right) / H_{\chi}\left(u^{s}-1\right) .
$$

For simplicity, we will write $G(T)=G_{\chi}(T)$ and $H(T)=H_{\chi}(T)$ when $\chi$ is a trivial character. By [DR], we know that $H(T)=1$ if $\chi$ is nontrivial; otherwise, $H_{\chi}(T)=T$. When $F=\mathbb{Q}$, it is known that the residue of the Riemann zeta function at 1 is 1 , so $G(T)$ is a unit by the following lemma. 
Lemma 5.2.1. A power series $f(T)$ is a unit in $\Lambda$ if and only if $f\left(u^{k}-1\right) \in \mathbb{Z}_{p}^{\times}$for some (equivalentlly, all) $k \in \mathbb{Z}_{\geq 0}$.

The proof of this lemma is straightforward, so we omit a proof here.

In general, when $F \neq \mathbb{Q}, G(T)$ may not ,be a unit in $\Lambda$ since the residue of the Dedekind zeta function at 1 may not be a $p$-adic unit.

Let $\chi_{1}$ and $\chi_{2}$ be narrow ideal class characters of conductor $\mathfrak{n}_{1}$ and $\mathfrak{n}_{2}$, respectively. We will always assume that $\chi_{1}$ is nontrivial. Recall that the infinite part of $\chi_{1}\left(\right.$ resp. $\left.\chi_{2}\right)$ is $\operatorname{sgn}^{q}\left(\operatorname{resp} \cdot \operatorname{sgn}^{r}\right)$ for $q \in(\mathbb{Z} / 2 \mathbb{Z})^{d}$ (resp. $\left.r \in(\mathbb{Z} / 2 \mathbb{Z})^{d}\right)$. Now we recall the construction of $\Lambda$-adic Eisenstein series.

Proposition 5.2.2. Let the notation be as above. Assume that $\mathfrak{n}_{1} \mathfrak{n}_{2}=\mathfrak{n}$ or $\mathfrak{n} p$ for some integral ideal $\mathfrak{n}$ prime to $p$, and assume that $q+r \equiv(0, \ldots, 0)\left(\bmod 2 \mathbb{Z}^{d}\right)$. Then there exists a $\Lambda$-adic Eisenstein series $\mathcal{E}\left(\chi_{1}, \chi_{2}\right) \in M\left(\mathfrak{n}, \chi_{1} \chi_{2} ; \Lambda\right)$ satisfying

$$
v_{k, \zeta}\left(\mathcal{E}\left(\chi_{1}, \chi_{2}\right)\right)=E_{k}\left(\chi_{1} \omega^{2-k} \rho_{\zeta}, \chi_{2}\right)
$$

Moreover, if $\left(\mathfrak{n}_{2}, p\right)=1$, then $\mathcal{E}\left(\chi_{1}, \chi_{2}\right) \in M^{\text {ord }}\left(\mathfrak{n} p, \chi_{1} \chi_{2} ; \Lambda\right)$.

Proof. We follow the argument in [Wil2, Proposition 1.3.1]. We define $\mathcal{E}\left(\chi_{1}, \chi_{2}\right)$ by setting

$$
C_{\lambda}\left(0, \mathcal{E}\left(\chi_{1}, \chi_{2}\right)\right)=2^{-d} G_{\chi_{1} \chi_{2}^{-1} \omega^{2}}\left(u^{2}(T+1)-1\right) / H_{\chi_{1} \chi_{2}^{-1} \omega^{2}}\left(u^{2}(T+1)-1\right)
$$

if $\chi_{2}$ is a trivial character and otherwise, setting $C_{\lambda}\left(0, \mathcal{E}\left(\chi_{1}, \chi_{2}\right)\right)=0$ for $\lambda=1, \ldots, h_{F}^{+}$. For an integral ideal $\mathfrak{m}$ of $\mathcal{O}_{F}$, the $\mathfrak{m}$-th Fourier coefficient is defined as

$$
C\left(\mathfrak{m}, \mathcal{E}\left(\chi_{1}, \chi_{2}\right)\right)=\sum_{\mathfrak{a} \mid \mathfrak{m}, p \nmid \mathfrak{a}} \overline{\chi_{1}}(\mathfrak{a}) \chi_{2}\left(\frac{\mathfrak{m}}{\mathfrak{a}}\right) N(\mathfrak{a}) .
$$

Recall that for any integral ideal $\mathfrak{a}$ of $\mathcal{O}_{F}$, we have $\overline{\chi_{1}}(\mathfrak{a})=\chi_{1}(\mathfrak{a})(1+T)^{s(\mathfrak{a})}$, where the number $s(\mathfrak{a}) \in \mathbb{Z}_{p}$ is defined by (5.1). Therefore, we have

$$
v_{k, \zeta}\left(\overline{\chi_{1}}(\mathfrak{a})\right)=\chi_{1}(\mathfrak{a}) \rho_{\zeta}(\langle N(\mathfrak{a})\rangle)\langle N(\mathfrak{a})\rangle^{k-2}=\chi_{1}(\mathfrak{a}) \rho_{\zeta}(\mathfrak{a}) \omega^{2-k}(\mathfrak{a}) N(\mathfrak{a})^{k-2}
$$


Thus we obtain

$$
v_{k, \zeta}\left(C\left(\mathfrak{m}, \mathcal{E}\left(\chi_{1}, \chi_{2}\right)\right)\right)=\sum_{\mathfrak{a} \mid \mathfrak{m}, p \nmid \mathfrak{a}} \chi_{1} \rho_{\zeta} \omega^{2-k}(\mathfrak{a}) \chi_{2}\left(\frac{\mathfrak{m}}{\mathfrak{a}}\right) N(\mathfrak{a})^{k-1}
$$

for all integral ideals $\mathfrak{m}$ of $\mathcal{O}_{F}$ prime to $\mathfrak{n} p$. The equality

$$
v_{k, \zeta}\left(C_{\lambda}\left(0, \mathcal{E}\left(\chi_{1}, \chi_{2}\right)\right)\right)=C_{\lambda}\left(0, E_{k}\left(\chi_{1} \omega^{2-k} \rho_{\zeta}, \chi_{2}\right)\right)
$$

for $\lambda=1, \ldots, h_{F}^{+}$follows from the interpolation property of the $p$-adic $L$-function.

The second assertion follows from the fact that for each prime ideal $\mathfrak{p}$ we have

$$
T(\mathfrak{p}) \mathcal{E}\left(\chi_{1}, \chi_{2}\right)=\left(\overline{\chi_{1}}(\mathfrak{p}) N(\mathfrak{p})+\chi_{2}(\mathfrak{p})\right) \mathcal{E}\left(\chi_{1}, \chi_{2}\right)
$$

For simplicity, we set $\widehat{G}_{\chi_{1} \chi_{2}^{-1}}(T):=G_{\chi_{1} \chi_{2}^{-1} \omega^{2}}\left(u^{2}(T+1)-1\right)$ and write it as $\widehat{G}(T)$ if $\left(\chi_{1}, \chi_{2}\right)=$ $\left(\omega^{-2}, \mathbf{1}\right)$. We will also write $\widehat{H}_{\chi}(T)$ and $\widehat{H}(T)$ in the same manner.

Now we fix an odd rational prime $p$ unramified in $F$ and fix an integral ideal $\mathfrak{n}$ of $F$ prime to $\mathfrak{D}$. As in Proposition 4.3.2, we write $\mathfrak{n}_{i}=\mathfrak{n}_{i}^{\prime} \times \mathfrak{m}_{i}$ for $i=1,2$. Also, we write the conductor of $\chi_{1}^{-1} \chi_{2}$ as $\mathfrak{n}_{1}^{\prime} \mathfrak{n}_{2}^{\prime} \mathfrak{m}$ for some integral ideal $\mathfrak{m}$ of $F$.

Proposition 5.2.3. Let the notation be as in Proposition 4.3.2. Assume that the assumption in Proposition 5.2.2 holds, and assume that $\left(\mathfrak{n}_{2}, p\right)=1$. Then the constant term of $\mathcal{E}\left(\chi_{1}, \chi_{2}\right)$ at the cusp $x_{\lambda} g=x_{\lambda}\left(\begin{array}{ll}a & b \\ c & d\end{array}\right)$

$$
\begin{aligned}
C \times(1+T)^{a\left(\frac{\mathfrak{m}_{1}}{\mathfrak{m} \mathfrak{n}_{2}^{\prime}}\right)} \chi_{2}\left(t_{\lambda} \mathfrak{D}\right) N\left(t_{\lambda} \mathfrak{D}\right)^{k / 2} \operatorname{sgn}^{q}\left(d_{\mathfrak{n}_{1}}\right) \overline{\chi_{1}}\left(d_{\mathfrak{n}_{1}}\right) \operatorname{sgn}^{r}\left(-c_{\mathfrak{n}_{2}} \mathfrak{m}_{1}^{-1}\right) \chi_{2}\left(c_{\mathfrak{n}_{2}} \mathfrak{m}^{-1}\right) \times \\
\prod_{\mathfrak{q} \mid \mathfrak{n}, \mathfrak{q} \nmid \operatorname{cond}\left(\chi_{1} \chi_{2}^{-1}\right)}\left(1-\chi_{1} \chi_{2}^{-1}(\mathfrak{q})(1+T)^{-a(\mathfrak{q})} N(\mathfrak{q})^{-2}\right) \widehat{G}_{\chi_{1} \chi_{2}^{-1}}(T)
\end{aligned}
$$

if $g$ satisfies the three conditions in Proposition 4.3.2. Here $C$ is a p-adic unit in a finite cyclotomic extension over $\mathbb{Q}_{p}$ only depending $\chi_{1}$ and $\chi_{2}$, and $s\left(\frac{\mathfrak{m}_{1}}{\mathfrak{m}_{2}^{\prime}}\right)$ and $s(\mathfrak{q})$ are defined by (5.1). 
PROOF. It follows from Proposition 4.3.2 that for $k \geq 2$, the constant term of $v_{k, 1}\left(\mathcal{E}\left(\chi_{1}, \chi_{2}\right)\right)=$ $E_{k}\left(\chi_{1} \omega^{2-k}, \chi_{2}\right)$ at the cusp $g$ is

$$
\begin{aligned}
& C_{1} \times \omega^{2-k}\left(\frac{\mathfrak{m}_{1}}{\mathfrak{m n n}_{2}^{\prime}}\right) \prod_{\mathfrak{q} \mid \mathfrak{n}, \mathfrak{q} \nmid \operatorname{cond}\left(\chi_{1} \chi_{2}^{-1}\right)}\left(1-\chi_{1} \omega^{2-k} \chi_{2}^{-1}(\mathfrak{q}) N(\mathfrak{q})^{-k}\right) L\left(1-k, \chi_{1} \omega^{2-k} \chi_{2}^{-1}\right) \times \chi_{2}\left(t_{\lambda} \mathfrak{D}\right) N\left(t_{\lambda} \mathfrak{D}\right)^{k / 2} \times \\
& \operatorname{sgn}^{q}\left(d_{\mathfrak{n}_{1}}\right) \chi_{1} \omega^{2-k}\left(d_{\mathfrak{n}_{1}}\right) \operatorname{sgn}^{r}\left(-c \mathfrak{m}_{1}^{-1}\right) \chi_{2}\left(c \mathfrak{m}^{-1}\right) \prod_{v \mid \mathfrak{n}_{2}} \varepsilon_{v}\left(2-k, \chi_{2, v}^{-1} \psi_{v}\right)^{-1} \times \prod_{v \mid \operatorname{gcd}\left(\mathfrak{n}_{1}, \mathfrak{n}_{2}\right)} \frac{\tau\left(\chi_{1, v}\right)}{\tau\left(\chi_{1, v}^{-1} \chi_{2, v}\right)}
\end{aligned}
$$

where $C_{1}$ is a $p$-adic unit in a finite cyclotomic extension of $\mathbb{Q}_{p}$ which only depends on $\chi_{1}$ and $\chi_{2}$. It is easy to see that $v_{k, 1}\left((1+T)^{a\left(\frac{\mathfrak{m}_{1}^{\prime}}{\mathfrak{m n}_{2}^{\prime}}\right)}\right)$ is a $p$-adic unit times $\omega^{2-k}\left(\frac{\mathfrak{m}_{1}}{\mathfrak{m n n}_{2}^{\prime}}\right)$ and that $v_{k, 1}\left(\overline{\chi_{1}}\right)(\mathfrak{a})$ is a $p$-adic unit times $\chi_{1} \omega^{2-k}(\mathfrak{a})$ for all integral ideals $\mathfrak{a}$ prime to $\mathfrak{n} p$. Moreover,we have

$$
v_{k, 1}\left(1-\chi_{1} \chi_{2}^{-1}(\mathfrak{q})(1+T)^{-a(\mathfrak{q})} N(\mathfrak{q})^{-2}\right)=1-\chi_{1} \omega^{2-k} \chi_{2}^{-1}(\mathfrak{q}) N(\mathfrak{q})^{-k}
$$

To complete the proof, it remains to show that $\prod_{v \mid \mathfrak{n}_{2}} \varepsilon_{v}\left(2-k, \chi_{2, v}^{-1}, \psi_{v}\right)$ and $\prod_{v \mid \operatorname{gcd}\left(\mathfrak{n}_{1}, \mathfrak{n}_{2}\right)} \frac{\tau\left(\chi_{1, v}\right)}{\tau\left(\chi_{1, v}^{-1} \chi_{2, v}\right)}$ are also $p$-adic units in a finite cyclotomic extension over $\mathbb{Q}_{p}$, which only depend on $\chi_{1}$ and $\chi_{2}$. This follows from Lemma 2.5.2(1) and (2), and the assumption that $\mathfrak{n}_{2}$ is prime to $p$.

Definition 5.2.4. Let the notation be as above. Assume that $\mathfrak{n}_{1} \mathfrak{n}_{2}=\mathfrak{n} p$ or $\mathfrak{n}$.

1. The Eisenstein ideal $\mathcal{I}\left(\chi_{1}, \chi_{2}\right)$ associated with the pair of characters $\left(\chi_{1}, \chi_{2}\right)$ is defined as the annihilator of $\mathcal{E}\left(\chi_{1}, \chi_{2}\right)$ in $\mathcal{H}^{\text {ord }}\left(\mathfrak{n}, \chi_{1} \chi_{2} ; \Lambda\right)$. We define $\mathfrak{M}\left(\chi_{1}, \chi_{2}\right)=\left(\mathcal{I}\left(\chi_{1}, \chi_{2}\right), \mathfrak{P}, T\right)$ to be the maximal ideal of $\mathcal{H}^{\text {ord }}\left(\mathfrak{n}, \chi_{1} \chi_{2} ; \Lambda\right)$ containing $\mathcal{I}\left(\chi_{1}, \chi_{2}\right)$, where $\mathfrak{P}$ is the maximal ideal of $\mathcal{O}_{\infty}$. We denote by $I\left(\chi_{1}, \chi_{2}\right)\left(\right.$ resp. $\left.m\left(\chi_{1}, \chi_{2}\right)\right)$ the image of $\mathcal{I}\left(\chi_{1}, \chi_{2}\right)\left(\right.$ resp. $\left.\mathfrak{M}\left(\chi_{1}, \chi_{2}\right)\right)$ in the cuspidal Hecke algebra $h^{\text {ord }}\left(\mathfrak{n}, \chi_{1} \chi_{2} ; \Lambda\right)$.

2. We say that a pair of narrow ideal class characters $\left(\chi_{1}, \chi_{2}\right)$ is not exceptional if the maximal ideal $\mathfrak{M}\left(\chi_{1}, \chi_{2}\right)$ does not contain any Eisenstein ideal other than $\mathcal{I}\left(\chi_{1}, \chi_{2}\right)$. 
Proposition 5.2.5. Let $\left(\chi_{1}, \chi_{2}\right)$ and $\left(\chi_{1}^{\prime}, \chi_{2}^{\prime}\right)$ be two pairs of narrow ray class characters of conductor $\mathfrak{n}_{i}$ and $\mathfrak{n}_{i}^{\prime}$ for $i=1,2$, respectively. Assume that $\mathfrak{n}_{1} \mathfrak{n}_{2}=\mathfrak{n}_{1}^{\prime} \mathfrak{n}_{2}^{\prime}=\mathfrak{n}$ or $\mathfrak{n} p$. Then the eigenvalues of $T(\mathfrak{q})$ of $\mathcal{E}\left(\chi_{1}, \chi_{2}\right)$ and $\mathcal{E}\left(\chi_{1}^{\prime}, \chi_{2}^{\prime}\right)$ are the same modulo $(\mathfrak{P}, T)$ for all prime ideals $\mathfrak{q}$ of $\mathcal{O}_{F}$ if and only if

$$
\left\{\begin{array}{l}
\chi_{1} \equiv \chi_{1}^{\prime} \text { and } \chi_{2} \equiv \chi_{2}^{\prime} \bmod \mathfrak{P} ; \text { or } \\
\chi_{1} \equiv \chi_{2}^{\prime} \omega^{-1}, \chi_{2} \equiv \chi_{1}^{\prime} \omega, \text { and } \chi_{1} \omega \chi_{2}^{-1}(\mathfrak{p}) \equiv 1 \bmod \mathfrak{P} \text { for all } \mathfrak{p} \mid p
\end{array}\right.
$$

If we assume further that $p \nmid \phi(N(\mathfrak{n})) h_{F}$, then the eigenvalues of $T(\mathfrak{q})$ of $\mathcal{E}\left(\chi_{1}, \chi_{2}\right)$ and $\mathcal{E}\left(\chi_{1}^{\prime}, \chi_{2}^{\prime}\right)$ are the same modulo $(\mathfrak{P}, T)$ for all prime ideals $\mathfrak{q}$ of $\mathcal{O}_{F}$ if and only if

$$
\left\{\begin{array}{l}
\chi_{1}=\chi_{1}^{\prime} \text { and } \chi_{2}=\chi_{2}^{\prime} ; \text { or } \\
\chi_{1}=\chi_{2}^{\prime} \omega^{-1}, \chi_{2}=\chi_{1}^{\prime} \omega, \text { and } \chi_{1} \omega \chi_{2}^{-1}(\mathfrak{p})=1 \text { for all } \mathfrak{p} \mid p .
\end{array}\right.
$$

Proof. We follow the argument in [Ohta3, Lemma 1.4.9]. For any prime ideal $\mathfrak{q}$ not dividing $\mathfrak{n} p$, we have

$$
\bar{\chi}_{1}(\mathfrak{q}) N(\mathfrak{q})+\chi_{2}(\mathfrak{q}) \equiv \bar{\chi}_{1}^{\prime}(\mathfrak{q}) N(\mathfrak{q})+\chi_{2}^{\prime}(\mathfrak{q}) \bmod (\mathfrak{P}, T)
$$

We obtain that

$$
\chi_{1}(\mathfrak{q}) N(\mathfrak{q})+\chi_{2}(\mathfrak{q}) \equiv \chi_{1}^{\prime}(\mathfrak{q}) N(\mathfrak{q})+\chi_{2}^{\prime}(\mathfrak{q}) \bmod \mathfrak{P}
$$

Since $N(\mathfrak{q}) \equiv \omega(\mathfrak{q}) \bmod \mathfrak{P}$, we have

$$
\chi_{1}(\mathfrak{q}) \omega(\mathfrak{q})+\chi_{2}(\mathfrak{q}) \equiv \chi_{1}^{\prime}(\mathfrak{q}) \omega(\mathfrak{q})+\chi_{2}^{\prime}(\mathfrak{q}) \bmod \mathfrak{P}
$$

Thus by Artins lemma on the linear independence of characters, we have

$$
\left\{\begin{array}{l}
\chi_{1} \omega \equiv \chi_{1}^{\prime} \omega \text { and } \chi_{2} \equiv \chi_{2}^{\prime} \bmod \mathfrak{P}, \text { or } \\
\chi_{1} \omega \equiv \chi_{2}^{\prime} \text { and } \chi_{2} \equiv \chi_{1}^{\prime} \omega \bmod \mathfrak{P} .
\end{array}\right.
$$

Next, we note that one can view all characters as narrow ray class characters modulo $\mathfrak{n} p$. Since the narrow ray class number $h_{F}^{+}(\mathfrak{m})=\left|\mathrm{Cl}_{F}^{+}(\mathfrak{m})\right|$ divides $2^{d} \phi(N(\mathfrak{m})) h_{F}$ for all integral ideals $\mathfrak{m}$ of $\mathcal{O}_{F}$, 
the assumption that $p \nmid \phi(N(\mathfrak{n})) h_{F}$ implies that the the field $\mathbb{Q}_{p}\left[\chi_{i}, \chi_{i}^{\prime} \mid i=1,2\right]$ is unramified over $\mathbb{Q}_{p}$. Therefore, by the Teichmüller lifting, we know that

$$
\left\{\begin{array}{l}
\chi_{1}=\chi_{1}^{\prime} \text { and } \chi_{2}=\chi_{2}^{\prime}, \text { or } \\
\chi_{1} \omega=\chi_{2}^{\prime} \text { and } \chi_{2}=\chi_{1}^{\prime} \omega .
\end{array}\right.
$$

To complete the proof, we recall that for all prime ideals $\mathfrak{p}$ dividing $p$, the $T(\mathfrak{p})$-eigenvalues of $\mathcal{E}\left(\chi_{1}, \chi_{2}\right)\left(\right.$ resp. $\left.\mathcal{E}\left(\chi_{1}^{\prime}, \chi_{2}^{\prime}\right)\right)$ are $\chi_{2}(\mathfrak{p})\left(\right.$ resp. $\left.\chi_{2}^{\prime}(\mathfrak{p})\right)$. Hence, we have $\chi_{1} \omega \chi_{2}^{-1}(\mathfrak{p}) \equiv 1 \bmod \mathfrak{P}$ and have $\chi_{1} \omega \chi_{2}^{-1}(\mathfrak{p})=1$ if $p \nmid \phi(N(\mathfrak{n})) h_{F}$ for all $\mathfrak{p} \mid p$.

\subsection{Control Theorem}

Let the notation be as in the previous section. In this subsection, we prove the control theorem for one-variable $\Lambda$-adic Hilbert modular forms under the assumption of Leopoldt's conjecture.

We first recall that the the $\Lambda$-adic Eisenstein series $\mathcal{E}=\mathcal{E}\left(\omega^{-2}, \mathbf{1}\right)$ has constant terms $\frac{1}{2^{d}} \zeta_{p}(1-s)=$ $\frac{\widehat{G}\left(u^{s}-1\right)}{2^{d} \widehat{H}\left(u^{s}-1\right)}$. Also, recall that Leopoldt's conjecture for $F$ asserts that the $p$-adic regulator of $F$ is nonzero. By the main result in $[\mathrm{Col}]$, we see that the non-vanishing of the $p$-adic regulator implies that the $p$-adic zeta function $\zeta_{p}(s)$ attached to $F$ has simple pole at 1 . Let $S=\left\{1, \widehat{G}, \widehat{G}^{2}, \ldots\right\}$, and let $\Lambda_{\widehat{G}}=S^{-1} \Lambda$ be the localization of $\Lambda$ at $S$. We set $\mathcal{E}^{\prime}=\frac{2^{d} T}{\widehat{G}} \mathcal{E}$. As we saw in the previous section that $\widehat{H}(T)=T$. Thus, $\mathcal{E}^{\prime}(0)=1$ and is a $\Lambda_{\widehat{G}^{-}}$adic modular form. The following lemma has been proved in $[\mathrm{Dpp}]$.

Proposition 5.3.1. Let the notation be as above. Assume that Leopoldt's conjecture for $F$ is true. Then for any $f \in M_{k}\left(K_{0}\left(\mathfrak{n} p^{r}\right), \chi \omega^{2-k} \rho ; \mathcal{O}_{\infty}\right)$, there exists a $\Lambda_{\widehat{G}^{-}}$adic modular form $\mathcal{F} \in M\left(\mathfrak{n}, \chi ; \Lambda_{\widehat{G}}\right)$ such that $v_{k, \zeta}(\mathcal{F})=f$. 
ProOF. We set $\mathcal{E}_{k}(T)=\mathcal{E}^{\prime}\left(\rho(u)^{-1} u^{2-k}(1+T)-1\right)$, which is a $\Lambda_{\widehat{G}^{-}}$adic modular form. Then we have

$$
\mathcal{E}_{k}\left(\rho(u) u^{k-2}-1\right)=\mathcal{E}^{\prime}(0)=1
$$

It is easy to see that $\mathcal{F}=\mathcal{E} \cdot f$ is a $\Lambda_{\widehat{G}^{-}}$adic modular form and $\mathcal{F}\left(\rho(u) u^{k-2}-1\right)=f$.

Corollary 5.3.2 (Control theorem). Let the notation be as above. Assume that Leopoldt's conjecture for $F$ is true. Then for almost all pairs of $(k, r)$, we have isomorphisms of $\Lambda_{\widehat{G}}$-modules

$$
\phi_{M}: M^{\text {ord }}\left(\mathfrak{n}, \chi ; \Lambda_{\widehat{G}}\right) /\left(T-\rho(u) u^{k-2}+1\right) \simeq M_{k}^{\text {ord }}\left(K_{0}\left(\mathfrak{n} p^{r}\right), \chi \omega^{2-k} \rho ; Q\left(\mathcal{O}_{\infty}\right)\right)
$$

and

$$
\phi_{S}: S^{\text {ord }}\left(\mathfrak{n}, \chi ; \Lambda_{\widehat{G}}\right) /\left(T-\rho(u) u^{k-2}+1\right) \simeq S_{k}^{\text {ord }}\left(K_{0}\left(\mathfrak{n} p^{r}\right), \chi \omega^{2-k} \rho ; Q\left(\mathcal{O}_{\infty}\right)\right)
$$

where $Q\left(\mathcal{O}_{\infty}\right)$ is the quotient field of $\mathcal{O}_{\infty}$. Consequently, $M^{\operatorname{ord}}\left(\mathfrak{n}, \chi ; \Lambda_{\widehat{G}}\right)$ and $S^{\text {ord }}\left(\mathfrak{n}, \chi ; \Lambda_{\widehat{G}}\right)$ are free $\Lambda_{\widehat{G}}$-modules of finite rank.

PROOF. In the case of $\Lambda$-adic cusp forms, the assertion was proved by Wiles [Wil2] without any assumption and without taking any localization. Here we give another proof under the assumption that Leopoldt's conjecture for $F$ is true. Since the proof for cusp forms is the same as the proof for modular forms, we only deal with the case of modular forms.

In [Wil2], Wiles has shown that for almost all pairs $(k, r)$, the homomorphism $\phi_{M}$ is an injection. By Proposition 5.3.1, we know that it is surjection. The second assertion follows from the first assertion by the following well-known lemma.

Lemma 5.3.3. Let $M$ be a finitely generated torsion free $\Lambda$-module. If $M / P$ is free for infinitely many height 1 prime ideals of $\Lambda$, then $M$ is free $\Lambda$-module of finite rank. 
For a proof, see [H2, Chapter 7].

In Chapter 6, we will prove Corollary 5.3.2 without assuming Leopoldt's conjecture and without taking any localization. 


\section{CHAPTER 6}

Hilbert modular varieties

Let $N$ be a positive integer, and let $\mathfrak{n}$ be a nonzero integral ideal in $\mathcal{O}_{F}$. We set

$$
\begin{gathered}
\Gamma(N)=\left\{\left(\begin{array}{ll}
a & b \\
c & d
\end{array}\right) \in \mathrm{GL}_{2}\left(\mathcal{O}_{F}\right)^{+} \mid b, c \in(N), a-1, d-1 \in(N)\right\}, \\
\Gamma_{1}(\mathfrak{n})=\left\{\left(\begin{array}{ll}
a & b \\
c & d
\end{array}\right) \in \mathrm{GL}_{2}\left(\mathcal{O}_{F}\right)^{+} \mid c \in \mathfrak{n}, d-1 \in \mathfrak{n}\right\}
\end{gathered}
$$

and

$$
\Gamma_{1}^{1}(\mathfrak{n})=\left\{\left(\begin{array}{ll}
a & b \\
c & d
\end{array}\right) \in \Gamma_{1}(\mathfrak{n}) \mid a-1 \in \mathfrak{n}\right\}
$$

In this section, we briefly review moduli problems with different level structures and their compactifications. Then we review the definition of $p$-adic modular forms. The main goal in the section is to prove a control theorem (Corollary 6.5.5) which is a key result in proving Lemma 7.1.1.

\subsection{Moduli problems with level structures}

In this subsection, we review moduli problems with different level structures. We refer the reader to [H3] and [Gor] for more information.

For simplicity, we denote by $\mathcal{O}=\mathcal{O}_{F}$ the ring of integers of $F$. Recall that an abelian scheme $A$ over a base scheme $S$ is a proper smooth geometrically irreducible group scheme over $S$. We denote by $A^{t}$ the dual abelian scheme of $A$. An $\mathcal{O}$-linear isogeny $\lambda: A \longrightarrow A^{t}$ is said to be symmetric if $\lambda=\lambda^{t}$. Let $\mathfrak{c}$ be a fractional ideal of $F$. A symmetric isogeny $\lambda$ is said to be a $\mathfrak{c}$-polarization if it induces an isomorphism $\lambda: A \otimes_{\mathcal{O}} \mathfrak{c} \simeq A^{t}$. Let $P(A)$ be the set of all $\mathcal{O}$-linear symmetric isogenies $\lambda: A \longrightarrow A^{t}$, 
which is a projective $\mathcal{O}$-module of rank one endowed with a natural notion of positivity. One has a homomorphism $A \otimes_{\mathcal{O}} P(A) \longrightarrow A^{t}$ which is determined uniquely by morphisms on $S$-algebras $T$ :

$$
\left(A \otimes_{\mathcal{O}} P(A)\right)(T)=A(T) \otimes_{\mathcal{O}} P(A) \longrightarrow A^{t}(T) ; x \otimes \lambda \mapsto \lambda(x) .
$$

We say that the abelian scheme $A$ satisfies the Deligne-Pappas condition if the above morphism is an isomorphism

$$
A \otimes_{\mathcal{O}} P(A) \simeq A^{t}
$$

If the discriminant $d_{F}$ of $F$ is invertible in $S$ or $\operatorname{char}(S)=0$, then the Deligne-Pappas condition is equivalent to the Rapoport condition [Gor, Lemma 5.5, p. 99]:

$$
\operatorname{Lie}(A) \text { is free } \mathcal{O} \otimes_{\mathbb{Z}} S \text {-module of rank } 1 .
$$

In general, the Rapoport condition (R) implies the Deligne-Pappas condition (DP) without any assumption [Gor, loc. cit.].

Definition 6.1.1. An abelian scheme $A$ with real multiplication (RM) by $\mathcal{O}$ over a scheme $S$ is an abelian scheme $A$ over $S$ of relative dimension $d=[F: \mathbb{Q}]$ together with an injection $\iota: \mathcal{O} \hookrightarrow$ $\operatorname{End}(A / S)$ such that $A$ satisfies the condition (DP).

For each positive integer $N$, a $\Gamma(N)$-level structure $\phi_{N}$ is an $\mathcal{O}$-linear isomorphism

$$
\phi_{N}:\left(\mathfrak{D}^{-1} \otimes_{\mathbb{Z}} \mu_{N}\right) \oplus\left(\mathcal{O} \otimes_{\mathbb{Z}} N^{-1} \mathbb{Z} / \mathbb{Z}\right) \simeq A[N]
$$

such that for any $(a \otimes \xi, b \otimes m) \in\left(\mathfrak{D}^{-1} \otimes_{\mathbb{Z}} \mu_{N}\right) \oplus\left(\mathcal{O} \otimes_{\mathbb{Z}} N^{-1} \mathbb{Z} / \mathbb{Z}\right)$, we have

$$
e_{N}\left(\phi_{N}(a \otimes \xi, b \otimes m), \lambda\left(\phi_{N}(a \otimes \xi, b \otimes m)\right)\right)=\langle a \otimes \xi, b \otimes m\rangle
$$

for some polarization $\lambda$. Here $e_{N}: A[N] \times A^{t}[N] \longrightarrow \mu_{N}$ is the Weill pairing and $\langle\cdot, \cdot\rangle:\left(\mathfrak{D}^{-1} \otimes_{\mathbb{Z}}\right.$ $\left.\mu_{N}\right) \oplus\left(\mathcal{O} \otimes_{\mathbb{Z}} N^{-1} \mathbb{Z} / \mathbb{Z}\right) \longrightarrow \mu_{N}$ is a natural pairing defined as $\langle a \otimes \xi, b \otimes m\rangle=e^{2 \pi i \operatorname{Tr}_{F / \mathbb{Q}}(a b)} \xi^{m}$. 
For each integral ideal $\mathfrak{n}$ of $F$, a $\mu_{\mathfrak{n}}$-level structure (also, called by $\Gamma_{1}^{1}(\mathfrak{n}$ )-level structure) on an abelian scheme $A / S$ with real multiplication by $\mathcal{O}$ is an $\mathcal{O}$-linear closed immersion

$$
\iota_{\mathfrak{n}}: \mu_{\mathfrak{n}} \otimes_{\mathbb{Z}} \mathfrak{D}^{-1} \hookrightarrow A
$$

of group schemes over $S$. Here $\mu_{\mathfrak{n} / \mathbb{Z}} \otimes_{\mathbb{Z}} \mathfrak{D}^{-1}$ is the $\mathfrak{n}$-torsion points of $\mathbb{G}_{m} \otimes_{\mathbb{Z}} \mathfrak{D}^{-1}$. That is

$$
\left(\mu_{\mathfrak{n}} \otimes_{\mathbb{Z}} \mathfrak{D}^{-1}\right)(R)=\left\{x \in \mathbb{G}_{m}(R) \otimes_{\mathbb{Z}} \mathfrak{D}^{-1} \mid \mathfrak{n} \cdot x=0\right\}
$$

for $\mathcal{O}$-algebra $R$. A $\mu_{p^{\infty}}$-level structure is a compatible sequence of $\mu_{p^{n}}$-level structures for $n \in \mathbb{Z}_{>0}$. Note that an abelian variety over a field of characteristic $p$ with real multiplication by $\mathcal{O}$ and a $\mu_{p^{n-}}$ level structure is ordinary in the sense that the connected component $A\left[p^{r}\right]^{\circ}$ of $A\left[p^{r}\right]$ is isomorphic to $\mu_{p^{r}}^{d}$ étale locally.

Definition 6.1.2. Let $\mathfrak{c}$ be a fractional ideal of $F$.

1. A morphism $f:(A, \iota, \lambda)_{/ S} \longrightarrow\left(A^{\prime}, \iota^{\prime}, \lambda^{\prime}\right)_{/ S}$ of abelian schemes with RM over a base scheme $S$ with $\mathfrak{c}$-polarizations is an $\mathcal{O}$-linear morphism $f: A_{/ S} \longrightarrow A_{/ S}^{\prime}$ of abelian schemes over $S$ such that $\iota^{\prime}=f \circ \iota$ and $\lambda=f^{t} \circ \lambda^{\prime} \circ f$, where $f^{t}: A^{\prime t} \longrightarrow A^{t}$ is the dual morphism of $f$.

2. A morphism $f:(A, \iota, \lambda)_{/ S} \longrightarrow\left(A^{\prime}, \iota^{\prime}, \lambda^{\prime}\right)_{/ S}$ is said to be an isomorphism if $f: A \longrightarrow A^{\prime}$ is an isomorphism.

3. Let $\eta$ be a $\Gamma(N)$-level structure or a $\mu_{\mathfrak{n}}$-level structure. A morphism $f:(A, \iota, \lambda, \eta)_{/ S} \longrightarrow$ $\left(A^{\prime}, \iota^{\prime}, \lambda^{\prime}, \eta^{\prime}\right)_{/ S}$ is a morphism $f:(A, \iota, \lambda)_{/ S} \longrightarrow\left(A^{\prime}, \iota^{\prime}, \lambda^{\prime}\right)_{/ S}$ satisfying $\eta^{\prime}=f \circ \eta$.

4. A morphism $f:(A, \iota, \lambda, \eta)_{/ S} \longrightarrow\left(A^{\prime}, \iota^{\prime}, \lambda^{\prime}, \eta^{\prime}\right)_{/ S}$ is said to be an isomorphism if $f: A_{/ S} \longrightarrow A_{/ S}^{\prime}$ is an isomorphism. 
We will omit the embedding $\iota: \mathcal{O} \hookrightarrow A$ from the tuple $(A, \iota, \lambda)$ if there is no confusion.

Let $\mathfrak{c}$ be a fractional ideal of $F$, and let $N \geq 3$ be a positive integer. The functor assigning to a $\mathbb{Z}\left[\frac{1}{N}\right]$-scheme $S$ the set of isomorphism classes of tuples $\left(A, \lambda, \phi_{N}\right)_{/ S}$ with $\mathfrak{c}$-polarization $\lambda$ is representable by a quasi-projective scheme $\mathfrak{M}(\mathfrak{c}, \Gamma(N))$ over $\mathbb{Z}\left[N^{-1}\right]$ which is smooth over $\mathbb{Z}\left[\left(N d_{F}\right)^{-1}\right]$ [Gor, Ch3, §6].

Let $\mathfrak{n}$ be an integral ideal of $F$ such that each test object $\left(A, \lambda, \iota_{\mathfrak{n}}\right)$ of $\Gamma_{1}^{1}(\mathfrak{n})$-level structure does not have any nontrivial automorphism (for example, $\mathfrak{n}$ is generated by a positive integer $N \geq 4$ ). The functor assigning to a $\mathbb{Z}$-scheme $S$ the set of isomorphism classes of tuples $\left(A, \lambda, \iota_{\mathfrak{n}}\right) / S$ (resp. $\left.\left(A,\left(\mathcal{O}^{\times}\right)^{+} \lambda, \iota_{\mathfrak{n}}\right)_{/ S}\right)$ is representable by a geometrically connected, quasi-projective scheme $\mathfrak{M}\left(\mathfrak{c}, \Gamma_{1}^{1}(\mathfrak{n})\right)$ $\left(\right.$ resp. $\left.\mathfrak{M}\left(\mathfrak{c}, \Gamma_{1}(\mathfrak{n})\right)\right)$ over $\mathbb{Z}$, which is smooth over $\mathbb{Z}\left[N(\mathfrak{n})^{-1}\right]$ [Gor, loc. cit.]. Note that the coarse moduli schemes $\mathfrak{M}\left(\mathfrak{c}, \Gamma_{1}^{1}(\mathfrak{n})\right)$ and $\mathfrak{M}\left(\mathfrak{c}, \Gamma_{1}(\mathfrak{n})\right)$ exist for all integral $\mathfrak{n}$. If one considers the Rapoport locus, then the above moduli problem is representable by a scheme over $\mathbb{Z}\left[\left(N d_{F}\right)^{-1}\right]$ (resp. $\mathbb{Z}\left[d_{F}^{-1}\right]$ ) for the $\Gamma(N)$-level (resp. $\mu_{\mathfrak{n}}$-level structure) and smooth over $\mathbb{Z}\left[\left(N d_{F}\right)^{-1}\right]\left(\right.$ resp. $\left.\mathbb{Z}\left[\left(N(\mathfrak{n}) d_{F}\right)^{-1}\right]\right)[$ Gor, loc. cit.].

Let $\mathcal{U}:=\left(\mathcal{O}^{\times}\right)^{+} /\left\{u^{2} \in \mathcal{O}^{\times} \mid u-1 \in \mathfrak{n}\right\}$, which acts on $\mathfrak{M}\left(\mathfrak{c}, \Gamma_{1}^{1}(\mathfrak{n})\right)$ by $u \cdot\left(A, \lambda, \iota_{\mathfrak{n}}\right)=\left(A, u \lambda, \iota_{\mathfrak{n}}\right)$ for $u \in E$. Indeed, $\mathfrak{M}\left(\mathfrak{c}, \Gamma_{1}(\mathfrak{n})\right)$ is the $\mathcal{U}$-invariant subscheme of $\mathfrak{M}\left(\mathfrak{c}, \Gamma_{1}^{1}(\mathfrak{n})\right)$, which follows from the following lemma.

Lemma 6.1.3. Let the notation be as above. If $u \in\left\{g^{2} \in \mathcal{O}^{\times} \mid g-1 \in \mathfrak{n}\right\}$, then $\left(A, \lambda, \iota_{\mathfrak{n}}\right) \longrightarrow$ $\left(A, u \lambda, \iota_{\mathfrak{n}}\right)$ is an isomorphism.

Proof. Given any $u \in \mathcal{O}^{\times}$, we consider the isomorphism $f_{u}: A \longrightarrow A$ defined by multiplying by $u$. It is easy to see that $f_{u}^{t} \circ \lambda \circ f_{u}=u^{2} \lambda$. Thus by Definition 6.1.2, we see that $f_{u}$ induces an isomorphism $f_{u}:(A, \lambda) \simeq\left(A, u^{2} \lambda\right)$. Moreover, it is also easy to see that $f_{u} \circ \iota_{\mathfrak{n}}=\iota_{\mathfrak{n}}$ if $u-1 \in \mathfrak{n}$. Hence, by 
Definition 6.1.2 again, we see that $f_{u}$ also induces an isomorphism $f_{u}:\left(A, \lambda, \iota_{\mathfrak{n}}\right) \simeq\left(A, u^{2} \lambda, \iota_{\mathfrak{n}}\right)$ if $u-1 \in \mathfrak{n}$. This completes the proof.

Lemma 6.1.4. Let $p$ be an odd rational prime. If $p \nmid \phi(N(\mathfrak{n}))$, then $p \nmid|\mathcal{U}|$.

PRoOF. Recall that we have shown in Section 2.1 that there is a natural isomorphism of groups

$$
(\mathcal{O} / \mathfrak{n})^{\times} \simeq F(\mathfrak{n}) / F_{\mathfrak{n}}
$$

Since $\left(\mathcal{O}^{\times}\right)^{+}$is a subgroup of $F(\mathfrak{n})$ and $\left(\mathcal{O}^{\times}\right)^{+} \cap F_{\mathfrak{n}}=\left\{u \in\left(\mathcal{O}^{\times}\right)^{+} \mid u-1 \in \mathfrak{n}\right\}$, we see that $\left(\mathcal{O}^{\times}\right)^{+} /\left\{u \in \mathcal{O}_{F}^{\times,+} \mid u-1 \in \mathfrak{n}\right\}$ is a subgroup of $F(\mathfrak{n}) / F_{\mathfrak{n}}$. Moreover, it is easy to see that the cardinality of $\left\{u \in\left(\mathcal{O}^{\times}\right)^{+} \mid u-1 \in \mathfrak{n}\right\} /\left\{u^{2} \mid u \in\left(\mathcal{O}^{\times}\right)^{+}, u-1 \in \mathfrak{n}\right\}$ is a power of 2 . Finally, we have

$$
|\mathcal{U}|=\left|\left(\mathcal{O}^{\times}\right)^{+} /\left\{u \in\left(\mathcal{O}^{\times}\right)^{+} \mid u-1 \in \mathfrak{n}\right\}\right| \times\left|\left\{u \in\left(\mathcal{O}^{\times}\right)^{+} \mid u-1 \in \mathfrak{n}\right\} /\left\{u^{2} \mid u \in\left(\mathcal{O}^{\times}\right)^{+}, u-1 \in \mathfrak{n}\right\}\right|
$$

which is a divisor of $\left|(\mathcal{O} / \mathfrak{n})^{\times}\right|=\phi(N(\mathfrak{n}))$ times a power of 2 . Thus, the assertion follows.

\subsection{Geometric modular forms}

In this section, we review the definition of geometric modular forms following [Gor]. Another useful reference is [H3]. Let $F^{\text {Gal }}$ be the Galois closure of $F$, and let $\mathcal{O}_{F^{\text {Gal }}}$ be the ring of integers of $F^{\text {Gal }}$. Let $T=\operatorname{Res}_{\mathcal{O} / \mathbb{Z}} \mathbb{G}_{m}$ be a torus over $\mathbb{Z}$. Throughout this section, we let $B$ be an $\mathcal{O}_{F}$ Gal $\left[d_{F}^{-1}\right]$-algebra. We put $T_{B}=T \times_{\operatorname{Spec}(\mathbb{Z})} \operatorname{Spec}(B)$. Then $T_{B}$ is a split torus. Note that one has a canonical isomorphism $T_{B} \simeq \mathbb{G}_{m / B}^{d}$ induced by the map

$$
\mathcal{O} \otimes_{\mathbb{Z}} B \simeq \bigoplus_{i=1}^{d} B ; \alpha \otimes 1 \mapsto\left(\tau_{1}(\alpha), \ldots, \tau_{d}(\alpha)\right)
$$

where $\left\{\tau_{1}, \ldots, \tau_{d}\right\}=I$ is fixed in Section 1.1. The character group $X\left(T_{B}\right)$ is a free abelian group generated by the $i$-th projection of $T_{B}$, denoted $\chi_{i}: T_{B}=\mathbb{G}_{m / B}^{d} \longrightarrow \mathbb{G}_{m / B}$ for $i=1, \ldots, d$. 
Let

$$
\mathfrak{M}=\mathfrak{M}\left(\mathfrak{c}, \Gamma_{1}^{1}(\mathfrak{n})\right) \longrightarrow \operatorname{Spec}\left(\mathcal{O}_{F \text { Gal }}\left[d_{F}^{-1}\right]\right)
$$

be the moduli scheme parameterizing tuples $\left(A, \lambda, \iota_{\mathfrak{n}}\right)$. Recall that the scheme $\mathfrak{M}\left(\mathfrak{c}, \Gamma_{1}^{1}(\mathfrak{n})\right)$ represents the functor that assigns to each $\mathcal{O}_{F \mathrm{Gal}}\left[d_{F}^{-1}\right]$-algebra $S$ the set of isomorphism classes of tuples $\left(A, \lambda, \iota_{\mathfrak{n}}\right)_{/ S}$ consisting of

- an abelian scheme $A \longrightarrow S$ with real multiplication by $\mathcal{O}$ such that the condition (DP) holds,

- a $\mathfrak{c}$-polarization $\lambda: A \longrightarrow A^{t}$,

- a $\mu_{\mathfrak{n}}$-level structure $\iota_{\mathfrak{n}}: \mu_{\mathfrak{n} / S} \otimes_{\mathbb{Z}} \mathfrak{D}^{-1} \hookrightarrow A$.

Let $\vartheta:\left(\mathcal{A}(\mathfrak{c}), \iota_{\mathfrak{n}}\right) \longrightarrow\left(\mathfrak{M}, \iota_{\mathfrak{n}}\right)$ be the universal abelian scheme with real multiplication by $\mathcal{O}$ with $\mu_{\mathfrak{n}}$-level structure [DT]. Let $\underline{\omega}=\operatorname{det} \vartheta_{*} \Omega_{\mathcal{A ( \mathfrak { c } ) / \mathfrak { M }}}$ be the determinant of the pushforward of the sheaf of relative differentials on $\mathcal{A}(\mathfrak{c})$. The action of $\left(\mathcal{O} \otimes_{\mathbb{Z}} B\right)^{\times}$on $\mathfrak{M}_{/ B}$ induces an action on $\underline{\omega}_{/ B}$, where $\mathfrak{M}_{/ B}$ is the base change of $\mathfrak{M}$ to $B$. One obtains a decomposition

$$
\underline{\omega}=\bigoplus_{i=1}^{d} \underline{\omega}\left(\chi_{i}\right) .
$$

For $\chi=\chi_{1}^{a_{1}} \cdots \chi_{d}^{a_{d}}$, we define

$$
\underline{\omega}(\chi)=\underline{\omega}\left(\chi_{1}\right)^{a_{1}} \otimes \cdots \otimes \underline{\omega}\left(\chi_{d}\right)^{a_{d}} .
$$

If $a_{1}=\cdots=a_{d}=k \in \mathbb{Z}$, we write $\underline{\omega}(\chi)=\underline{\omega}^{k}$ which is defined over $\mathbb{Z}\left[d_{F}^{-1}\right]$ instead of over $\mathcal{O}_{F}{ }_{\text {Gal }}\left[d_{F}^{-1}\right]$. When $k=1$, we will write $\underline{\omega}(\chi)=\underline{\omega}^{1}$ to indicate that it is $\operatorname{different}$ from $\underline{\omega}=\operatorname{det} \vartheta_{*} \Omega_{\mathcal{A}(\mathfrak{c}) / \mathfrak{M}}$. For our purpose (Section 6.5 ), we will only use $\underline{\omega}^{k}$ for $k \in \mathbb{Z}_{\geq 2}$.

Definition 6.2.1. Let the notation be as above. A $\mathfrak{c}$-Hilbert modular form $f$ over $B$ of level $\Gamma_{1}^{1}(\mathfrak{n})$ (or $\left.\mu_{\mathfrak{n}}\right)$ and weight $\chi \in X\left(T_{B}\right)$ is a global section of $\underline{\omega}(\chi)$ on $\mathfrak{M}\left(\mathfrak{c}, \Gamma_{1}^{1}(\mathfrak{n})\right)$. 
By Definition 6.2.1, we know that the space of $\mathfrak{c}$-Hilbert modular forms over $B$ of level $\Gamma_{1}^{1}(\mathfrak{n})$ and weight $\chi$ is $H^{0}\left(\mathfrak{M}\left(\mathfrak{c}, \Gamma_{1}^{1}(\mathfrak{n})\right)_{/ B}, \underline{\omega}(\chi)\right)$. One can define the spaces of $\mathfrak{c}$-Hilbert modular forms of level $\Gamma(N)$ and $\Gamma_{1}(\mathfrak{n})$ in the same manner. We denote by $H^{0}\left(\mathfrak{M}(\mathfrak{c}, \Gamma(N))_{/ B}, \underline{\omega}(\chi)\right)$ and $H^{0}\left(\mathfrak{M}\left(\mathfrak{c}, \Gamma_{1}(\mathfrak{n})\right)_{/ B}, \underline{\omega}(\chi)\right)$ the corresponding spaces.

By the discussion in the previous section, we know that for each test object in $\mathfrak{M}_{/ B}$, the Rapoport condition also holds, i.e., $\underline{\omega}$ is a free $\mathcal{O} \otimes_{\mathbb{Z}} B$-module of rank 1 . A generator $\omega \in \underline{\omega}$ is called a nonvanishing differential. The following definition is an equivalent definition of $\mathfrak{c}$-Hilbert modular forms (see $[$ Gor $]$ or $[\mathrm{H} 3])$.

Definition 6.2.2. Let the notation be as above. A $\mathfrak{c}$-Hilbert modular form $f$ over $B$ of level $\Gamma_{1}^{1}(\mathfrak{n})$ and weight $\chi$ is a rule

$$
\left(A, \lambda, \iota_{\mathfrak{n}}, \omega\right) \mapsto f\left(A, \lambda, \iota_{\mathfrak{n}}, \omega\right) \in B
$$

satisfying the following properties:

1. $f$ commutes with the base change,

2. $f$ depends only on the isomorphism class of $\left(A, \lambda, \iota_{\mathfrak{n}}, \omega\right)_{/ B}$,

3. for $\alpha \in(\mathcal{O} \otimes B)^{\times}=T_{B}$, we have

$$
f\left(A, \lambda, \iota_{\mathfrak{n}}, \alpha^{-1} \omega\right)=\chi(\alpha) f\left(A, \lambda, \iota_{\mathfrak{n}}, \omega\right)
$$

We denote by $G_{\chi}\left(\mathfrak{c}, \Gamma_{1}^{1}(\mathfrak{n}) ; B\right)$ the space of $\mathfrak{c}$-Hilbert modular forms over $B$ of weight $\chi$ and level $\Gamma_{1}^{1}(\mathfrak{n})$. In particular, when $a_{1}=\cdots=a_{d}=k$, we denote by $G_{k}\left(\mathfrak{c}, \Gamma_{1}^{1}(\mathfrak{n}) ; B\right)$ the space of modular forms, which is known as the space of modular forms of parallel weight $k$.

For $\Gamma=\Gamma(N)$ or $\Gamma_{1}(\mathfrak{n})$, one can define $G_{\chi}(\mathfrak{c}, \Gamma)$ in the same manner. We will also denote by $G_{k}(\mathfrak{c}, \Gamma)$ the space of modular forms of parallel weight $k$. 
For $\Gamma=\Gamma(N)$ or $\Gamma_{1}^{1}(\mathfrak{n})$ or $\Gamma_{1}(\mathfrak{n})$, by the above two definitions, we obtain the equality

$$
H^{0}\left(\mathfrak{M}(\mathfrak{c}, \Gamma)_{/ B}, \underline{\omega}(\chi)\right)=G_{\chi}(\mathfrak{c}, \Gamma ; B)
$$

and in particular,

$$
H^{0}\left(\mathfrak{M}(\mathfrak{c}, \Gamma)_{/ B}, \underline{\omega}^{k}\right)=G_{k}(\mathfrak{c}, \Gamma ; B)
$$

for $k \in \mathbb{Z}$. From the discussion in Section 6.1, we see that

$$
G_{\chi}\left(\mathfrak{c}, \Gamma_{1}(\mathfrak{n})\right)=G_{\chi}\left(\mathfrak{c}, \Gamma_{1}^{1}(\mathfrak{n})\right)^{\mathcal{U}}
$$

In particular, it follows from Lemma 6.1.4 that $G_{k}\left(\mathfrak{c}, \Gamma_{1}(\mathfrak{n}) ; \mathbb{Z}_{p}\right)$ is a direct summand of $G_{k}\left(\mathfrak{c}, \Gamma_{1}^{1}(\mathfrak{n}) ; \mathbb{Z}_{p}\right)$ if $p$ is an odd rational prime unramified in $F$ not and dividing $\phi(N(\mathfrak{n}))$.

Let $f \in G_{\chi}\left(\mathfrak{c}, \Gamma_{1}(\mathfrak{n}) ; B\right)$, and let $\chi_{0}:(\mathcal{O} / \mathfrak{n} \mathcal{O})^{\times} \longrightarrow B$ be a character of finite order. We say that $f$ is of type $\chi_{0}$ if

$$
a \cdot f\left(A, \lambda, \iota_{\mathfrak{n}}, \omega\right)=f\left(A, \lambda, a \iota_{\mathfrak{n}}, \omega\right)=\chi_{0}(a) f
$$

for $a \in(\mathcal{O} / \mathfrak{n O})^{\times}$. We denote by $G_{\chi}\left(\mathfrak{c}, \mathfrak{n}, \chi_{0} ; B\right)$ and $H^{0}\left(\mathfrak{M}\left(\mathfrak{c}, \Gamma_{1}(\mathfrak{n})\right), \underline{\omega}(\chi)\right)\left[\chi_{0}\right]$ the space of $\mathfrak{c}$-Hilbert modular forms of level $\Gamma_{1}(\mathfrak{n})$, type $\chi_{0}$, and weight $\chi$.

When $B=\mathbb{C}$, it is known $[\mathrm{H} 3, \S 4.1 .3]$ that there are canonical isomorphisms

$$
M_{k}\left(\Gamma_{\mathfrak{c}, \mathfrak{n}} ; \mathbb{C}\right) \simeq G_{k}(\mathfrak{c}, \Gamma ; \mathbb{C})
$$

for $\Gamma=\Gamma_{1}(\mathfrak{n})$, and $\Gamma_{1}^{1}(\mathfrak{n})$. Here $\Gamma_{\mathfrak{c}, \mathfrak{n}}=\Gamma_{1}^{1}(\mathfrak{c}, \mathfrak{n})\left(\operatorname{resp} . \Gamma_{1}(\mathfrak{c}, \mathfrak{n})\right)$ if $\Gamma=\Gamma_{1}^{1}(\mathfrak{n})\left(\operatorname{resp} . \Gamma=\Gamma_{1}(\mathfrak{n})\right)$. This isomorphism is obtained by the fact that for all $z \in \mathbf{H}^{d}$, one can construct an complex abelian variety $A_{z}$ with RM by $\mathcal{O}$, and all abelian complex varieties with RM by $\mathcal{O}$ are of this form. Here $\mathbf{H}$ is the complex upper half plane.

Now, we fix an odd rational prime $p$ unramified in $F$. Let $\mathfrak{n}$ be an integral ideal prime to $p$. Recall that Deligne and Ribet $[\mathrm{DR}]$ proved that $q$-expansion principle holds for modular forms of 
level $\Gamma_{1}^{1}(\mathfrak{n})$, and hence, it also holds for modular forms of level $\Gamma_{1}(\mathfrak{n})$. It asserts that the $q$-expansion of a modular form at the cusp $\infty$ determines the modular form, i.e., if all of the coefficients of $f$ are in a $\mathbb{Z}_{p}$-algebra $R$, then $f \in G_{k}\left(\mathfrak{c}, \Gamma_{1}(\mathfrak{n}) ; R\right)$. Thus, we have the following isomorphisms:

$$
M_{k}\left(\Gamma_{1}(\mathfrak{c}, \mathfrak{n}) ; R\right) \simeq G_{k}\left(\mathfrak{c}, \Gamma_{1}(\mathfrak{n}) ; R\right), \text { and } M_{k}\left(\Gamma_{1}^{1}(\mathfrak{c}, \mathfrak{n}) ; R\right) \simeq G_{k}\left(\mathfrak{c}, \Gamma_{1}^{1}(\mathfrak{n}) ; R\right)
$$

Let $\left\{t_{1}, \ldots, t_{h_{F}^{+}}\right\}$be a fixed set of representatives of $\mathrm{Cl}_{F}^{+}$such that $t_{\lambda}$ is coprime to $p$ for all $\lambda=1, \ldots,, h_{F}^{+}$. We set

$$
G_{k}(\mathfrak{n} ; R):=\bigoplus_{\lambda=1}^{h_{F}^{+}} G_{k}\left(t_{\lambda} \mathfrak{D}, \Gamma_{1}(\mathfrak{n}) ; R\right) .
$$

The space $G_{k}(\mathfrak{n} ; R)$ coincides with the space $M_{k}\left(K_{1}(\mathfrak{n}) ; R\right)$ defined in Chapter 3 .

In Chapter 3, we reviewed the Hecke action on $M_{k}\left(K_{1}(\mathfrak{n}) ; R\right)$. This action induces a Hecke action on $G_{k}(\mathfrak{n} ; R)$. One can also define the action geometrically (see [H3, Ch 4] for the definition). Let $e^{\circ}=\lim _{n \rightarrow \infty} \prod_{\mathfrak{p} \mid p} T(\mathfrak{p})^{n !}$, and let $e=\lim _{n \rightarrow \infty} \prod_{\mathfrak{p} \mid p} U(\mathfrak{p})^{n !}$. We set

$$
G_{k}^{\text {ord }}(\mathfrak{n} ; R)=e^{\circ} \cdot G_{k}(\mathfrak{n} ; R)
$$

and set

$$
G_{k}^{\text {ord }}\left(\mathfrak{n} p^{r} ; R\right)=e \cdot G_{k}\left(\mathfrak{n} p^{r} ; R\right)
$$

for $r \in \mathbb{Z}_{>0}$. Then we have

$$
G_{k}^{\text {ord }}\left(\mathfrak{n} p^{r} ; R\right)=M_{k}^{\text {ord }}\left(K_{1}\left(\mathfrak{n} p^{r}\right) ; R\right)
$$

for all $r \in \mathbb{Z}_{\geq 0}$. Note that when $r>0$, we only obtain the space of ordinary modular forms since all the test objects are ordinary modulo primes dividing $p$. We also define $G_{k}^{\text {ord }}(\mathfrak{c}, \Gamma ; R)$ for $\Gamma=\Gamma(N)$, $\Gamma_{1}^{1}(\mathfrak{n})$, and $\Gamma_{1}(\mathfrak{n})$ in the same manner. 


\subsection{Toroidal compactification}

Let $\mathfrak{M}$ be $\mathfrak{M}(\mathfrak{c}, \Gamma(N)), \mathfrak{M}\left(\mathfrak{c}, \Gamma_{1}^{1}(\mathfrak{n})\right)$, or $\mathfrak{M}\left(\mathfrak{c}, \Gamma_{1}(\mathfrak{n})\right)$. The existence of toroidal compactifications of Hilbert-Blumenthal varieties has been proved in [Rap] for $\mathfrak{M}=\mathfrak{M}(\mathfrak{c}, \Gamma(N))$ and in $[\operatorname{Dim} 1]$ for $\mathfrak{M}=\mathfrak{M}\left(\mathfrak{c}, \Gamma_{1}^{1}(\mathfrak{n})\right)$ and $\mathfrak{M}\left(\mathfrak{c}, \Gamma_{1}(\mathfrak{n})\right)$

To a smooth rational cone decomposition $\Sigma$ of $F^{+}$(see loc. cit. for the definition), one can attach the toroidal compactification $M=M_{\Sigma}$, which is proper smooth scheme over $\mathbb{Z}\left[\frac{1}{N(\mathfrak{n})}\right]$ when $\left.\mathfrak{M}=\Gamma_{1}^{1}(\mathfrak{n})\right)$ and $\mathfrak{M}\left(\mathfrak{c}, \Gamma_{1}(\mathfrak{n})\right)$ (or over $\mathbb{Z}\left[\frac{1}{N}\right]$ when $\mathfrak{M}=\mathfrak{M}(\mathfrak{c}, \Gamma(N))$ ) containing $\mathfrak{M}$ as an open dense subscheme. The boundary $M-\mathfrak{M}$ is a divisor with normal crossing. Moreover, there is a tuple $(\mathcal{G}, \lambda, \eta)$ over $M$, where $\pi: \mathcal{G} \longrightarrow M$ is a semi-abelian scheme with $\mathcal{O}$-action, $\lambda: \mathcal{G} \longrightarrow \mathcal{G}^{t}$ is a homomorphism such that the pullback of $\mathfrak{M} \subset M$ in $\mathcal{G}$ is $\mathcal{A}(\mathfrak{c})$, and $\eta$ is the corresponding level structure. We also denote by $\underline{\omega}=\operatorname{det} \pi_{*} \Omega_{\mathcal{G} / M}$ the determinant of the sheaf of relative differentials on $M$, which extends the sheaf of relative differentials on $\mathfrak{M}$ [loc. cit.].

Let $B$ be an $\mathcal{O}_{F \text { Gal }}\left[\frac{1}{d_{F}}\right]$-algebra. The action of $(\mathcal{O} \otimes B)^{\times}$on $\underline{\omega}_{/ B}$ induces a decomposition $\underline{\omega}(\chi)=$ $\underline{\omega}\left(\chi_{1}\right)^{a_{1}} \otimes \cdots \otimes \underline{\omega}\left(\chi_{d}\right)^{a_{d}}$. We denote by $\underline{\omega}^{k}$ the sheaf of parallel weight $k$ for $k \in \mathbb{Z}$. Koecher's principle $[H 3, \S 4.1 .4]$ tells us that for each positive integer $k$, if $F \neq \mathbb{Q}$, one has

$$
H^{0}\left(M, \underline{\omega}^{k}\right)=H^{0}\left(\mathfrak{M}, \underline{\omega}^{k}\right)
$$

Therefore, $H^{0}\left(M, \underline{\omega}^{k}\right)$ is independent of choice of the cone decomposition $\Sigma$ for all positive integers $k$.

\subsection{Minimal compactification}

Let the notation be in the previous section. From now on, we fix an odd rational prime $p$ unramified in $F$ and fix an integral ideal $\mathfrak{n}$ prime to $p$. The minimal compactification of $\mathfrak{M}$ was established in 
$\left[\right.$ Cha] for $\mathfrak{M}=\mathfrak{M}(\mathfrak{c}, \Gamma(N))$ and in $[\operatorname{Dim} 1]$ for $\mathfrak{M}=\mathfrak{M}\left(\mathfrak{c}, \Gamma_{1}^{1}(\mathfrak{n})\right)$ and $\mathfrak{M}\left(\mathfrak{c}, \Gamma_{1}(\mathfrak{n})\right)$

We put $\mathcal{O}_{\mathcal{M}}=\bigoplus_{k \geq 0} H^{0}\left(\mathfrak{M}, \underline{\omega}^{k}\right)$ and define $M^{*}:=\operatorname{Proj}\left(\mathcal{O}_{\mathcal{M}}\right)$. On $\mathbb{C}$-points, it is the minimal compactification of $\mathfrak{M}$ by adjoining one point at each cusp, i.e., $M^{*}(\mathbb{C})=\mathfrak{M}(\mathbb{C}) \cup\{$ cusps $\}$. In addition, the invertible sheaf $\underline{\omega}$ on $\mathfrak{M}$ extends to an ample line bundle [H3, §4.1.6], also denoted by $\underline{\omega}$, on $M^{*}$. Thus for $a \in \mathbb{Z}_{>0}$ big enough, we have

$$
H^{0}\left(M_{/ W}^{*}, \underline{\omega}_{/ W}^{a(p-1)}\right) \otimes_{W} \mathbb{F}=H^{0}\left(M_{/ \mathbb{F}}^{*}, \underline{\omega}_{/ \mathbb{F}}^{a(p-1)}\right),
$$

where $W$ is a finite extension of $\mathbb{Z}_{p}$ and $\mathbb{F}$ is the residue field of $W$. One obtains a lifting $E \in$ $H^{0}\left(M_{/ W}^{*}, \underline{\omega}_{/ W}^{a(p-1)}\right)$ of $H^{a}$, where $H \in H^{0}\left(M_{/ \mathbb{F}}^{*}, \underline{\omega}_{/ \mathbb{F}}^{(p-1)}\right)$ is the Hasse invariant. Recall that the Hasse invariant $H$ satisfies the property that

$$
H \equiv 1 \bmod p
$$

See $\left[\right.$ H3, loc. cit.] for more details of $H$. Since the Hasse invariant is a nontrivial section on $\underline{\omega}^{p-1}$, the ordinary locus of $M^{*}$, which is denoted by $S^{*}=M^{*}\left[\frac{1}{E}\right] \subset M^{*}$, is defined by $S^{*}:=\operatorname{Spec}\left(\mathcal{O}_{\mathcal{M}} /(E-1)\right)$ [H3, loc. cit.]. Moreover, it is affine and irreducible. We put $S=M\left[\frac{1}{E}\right]$ and $\mathfrak{S}=\mathfrak{M}\left[\frac{1}{E}\right]$. Moreover, for $r \in \mathbb{Z}_{>0}$, one can view $E$ as an element in $H^{0}\left(\mathfrak{M}\left(\mathfrak{c}, \Gamma_{1}\left(\mathfrak{n} p^{r}\right)\right), \underline{\omega}^{a(p-1)}\right)$ via the embedding

$$
H^{0}\left(M\left(\mathfrak{c}, \Gamma_{1}(\mathfrak{n})\right), \underline{\omega}_{/ W}^{a(p-1)}\right) \hookrightarrow H^{0}\left(M\left(\mathfrak{c}, \Gamma_{1}\left(\mathfrak{n} p^{r}\right)\right), \underline{\omega}_{/ W}^{a(p-1)}\right)
$$

We set $\mathfrak{S}\left(\mathfrak{c}, \Gamma_{1}\left(\mathfrak{n} p^{r}\right)\right)=\mathfrak{M}\left(\mathfrak{c}, \Gamma_{1}\left(\mathfrak{n} p^{r}\right)\right)\left[\frac{1}{E}\right]$. Note that $S$ and $\mathfrak{S}$ are not affine.

Recall that from the main theorem of [Cha], we have a canonical morphism

$$
\pi: M(\mathfrak{c}, \Gamma(N)) \longrightarrow M^{*}(\mathfrak{c}, \Gamma(N))
$$

which induces a canonical morphism $\pi: S(\mathfrak{c}, \Gamma(N)) \longrightarrow S^{*}(\mathfrak{c}, \Gamma(N))$.

Lemma 6.4.1. Let $M=M(\mathfrak{c}, \Gamma(N))$. Then we have

$$
\pi_{*}\left(\underline{\omega}_{/ S}^{k}\right) \otimes_{W} W_{m}=\pi_{*}\left(\underline{\omega}_{/ S}^{k} \otimes_{W} W_{m}\right)
$$


Proof. We follow the argument of [H3, p.120]. Since $\pi$ is an isomorphism outside $S-\mathfrak{S}$, it suffices to show the assertion on the stalk at each cusp. Let $R$ be a $\mathbb{Z}\left[\frac{1}{N}, \mu_{N}\right]$-algebra. For a fractional ideal $\mathfrak{a}$ of $F$, we set $\mathfrak{a}_{\geq 0}=\{0\} \cup \mathfrak{a}^{+}$. Recall that $\mathfrak{a}^{+}$is the subset of totally positive elements of $\mathfrak{a}$. Let $R\left[\left|\frac{1}{N}(\mathfrak{a})_{\geq 0}\right|\right]$ be the set of all formal power series $a_{0}+\sum_{\xi \in \frac{1}{N}(\mathfrak{a})^{+}} a_{\xi} q^{\xi}$ such that $a_{u^{2} \xi}=a_{\xi}$ for all $u \in \mathcal{O}^{\times}$ with $u-1 \in(N)$. Recall that $u \in \mathcal{O}^{\times}$acts on formal power series $\sum_{\xi \in \frac{1}{N}(\mathfrak{a}) \geq 0} a(\xi) q^{\xi}$ by

$$
u \cdot\left(\sum_{\xi \in \frac{1}{N}(\mathfrak{a}) \geq 0} a(\xi) q^{\xi}\right)=\sum_{\xi \in \frac{1}{N}(\mathfrak{a})_{\geq 0}} u^{-k} a\left(u^{2} \xi\right) q^{\xi} .
$$

Let $U_{N}=\left\{u^{2} \mid g \in \mathcal{O}^{\times}, u-1 \in(N)\right\}$. For each cusp $c$ associated with the ideals $\mathfrak{a b}^{-1}=\mathfrak{c}$, by [Cha, Theorem 3.6], one has

$$
\begin{aligned}
& \widehat{\pi_{*}\left(\underline{\omega}_{/ R}^{k}\right)_{c}} \simeq H^{0}\left(U_{N}, R\left[\left|\frac{1}{N}(\mathfrak{a} \mathfrak{b})_{\geq 0}\right|\right]\right) \\
= & \left\{\sum_{\xi \in \frac{1}{N}(\mathfrak{a} \mathfrak{b})_{\geq 0}} a(\xi) q^{\xi} \in R\left[\left|\frac{1}{N}(\mathfrak{a} \mathfrak{b})_{\geq 0}\right|\right]: a\left(u^{2} \xi\right)=N_{F / \mathbb{Q}}(u)^{k} a(\xi) \text { for all } u \in U_{N}\right\} \\
= & { }^{*)}\left\{\sum_{\xi \in \frac{1}{N}(\mathfrak{a} \mathfrak{b})_{\geq 0}} a(\xi) q^{\xi} \in R\left[\left|\frac{1}{N}(\mathfrak{a} \mathfrak{b})_{\geq 0}\right|\right]: a\left(u^{2} \xi\right)=a(\xi) \text { for all } u \in U_{N}\right\} \\
= & R\left[\left|\frac{1}{N}(\mathfrak{a} \mathfrak{b})_{\geq 0}\right|\right] .
\end{aligned}
$$

The equality $(*)$ follows from the fact that $N_{F / \mathbb{Q}}(u)^{k}=1$ because $u \in U_{N}$. Therefore, the short exact of sequence of $U_{N}$-modules

$$
0 \longrightarrow R\left[\left|\frac{1}{N}(\mathfrak{a} \mathfrak{b})_{\geq 0}\right|\right] \stackrel{p^{m}}{\longrightarrow} R\left[\left|\frac{1}{N}(\mathfrak{a} \mathfrak{b})_{\geq 0}\right|\right] \longrightarrow R / p^{m}\left[\left|\frac{1}{N}(\mathfrak{a} \mathfrak{b})_{\geq 0}\right|\right] \longrightarrow 0
$$

induces a short exact sequence

$$
0 \longrightarrow H^{0}\left(U_{N}, R\left[\left|\frac{1}{N}(\mathfrak{a} \mathfrak{b})_{\geq 0}\right|\right]\right) \stackrel{p^{m}}{\longrightarrow} H^{0}\left(U_{N}, R\left[\left|\frac{1}{N}(\mathfrak{a} \mathfrak{b})_{\geq 0}\right|\right]\right) \longrightarrow H^{0}\left(U_{N}, R / p^{m}\left[\left|\frac{1}{N}(\mathfrak{a} \mathfrak{b})_{\geq 0}\right|\right]\right) \longrightarrow 0
$$

because these are the same groups as above. Then the assertion follows. 
Corollary 6.4.2. Let the notation be as in Lemma 6.4.1, and let $W_{m}:=W / p^{m} W$. Then we have

$$
H^{0}\left(S_{/ W}, \underline{\omega}^{k}\right) \otimes_{W} W_{m} \simeq H^{0}\left(S_{/ W}, \underline{\omega}^{k} \otimes_{W} W_{m}\right)
$$

Proof. By definition, we have $H^{0}\left(S, \underline{\omega}^{k}\right) \otimes_{W} W_{m}=H^{0}\left(S^{*}, \pi_{*}\left(\underline{\omega}_{/ S}^{k}\right)\right) \otimes_{W} W_{m}$. Since $S^{*}$ is affine, $H^{0}\left(S^{*}, \pi_{*}\left(\underline{\omega}_{/ S}^{k}\right)\right) \otimes_{W} W_{m}=H^{0}\left(S^{*}, \pi_{*}\left(\underline{\omega}_{/ S}^{k}\right) \otimes_{W} W_{m}\right)$, and hence, the assertion follows from Lemma 6.4.1.

\section{$6.5 p$-adic modular forms}

In this section, we review the definition of $p$-adic modular forms following $[\mathrm{H} 3, \S 4.1]$ and prove a control theorem for later use. Let $\Gamma$ be $\Gamma(N), \Gamma_{1}^{1}(\mathfrak{n})$, or $\Gamma_{1}(\mathfrak{n})$, and let $\Gamma_{1}^{r}=\Gamma \cap \Gamma_{1}\left(p^{r}\right)$ (resp. $\left.\Gamma_{0}^{r}=\Gamma \cap \Gamma_{0}\left(p^{r}\right)\right)$. Let $W$ and $W_{m}$ be as in the previous section. Let $T_{m, n}(\mathfrak{c}, \Gamma)_{/ W_{m}}$ be the Hilbert modular Igusa tower (see $[\mathrm{H} 3, \S 4.1 .6]$ for the definition) which represents the functor that assigns to

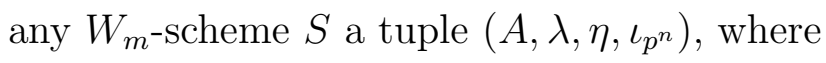

- $A \longrightarrow S$ is an abelian scheme with RM by $\mathcal{O}$ of relative dimension $d$,

- $\lambda: A \longrightarrow A^{t}$ is a $\mathfrak{c}$-polarization,

- $\eta$ is a $\Gamma$-level structure. That is, if $\Gamma=\Gamma(N)$, then $\eta=\phi_{N}$, and if $\Gamma=\Gamma_{1}^{1}(\mathfrak{n})$, then $\eta=\iota_{\mathfrak{n}}$.

- $\iota_{p^{n}}: \mu_{p^{n}} \otimes_{\mathbb{Z}} \mathfrak{D}^{-1} \hookrightarrow A$ is an inclusion of group schemes.

It was proved by Deligne-Ribet $[\mathrm{DR}]$ (also see $[\mathrm{H} 3, \S 4.2]$ ) that $T_{m, n}\left(\mathfrak{c}, \Gamma_{1}^{1}(\mathfrak{n})\right.$ ) is geometrically irreducible for any integral ideal $\mathfrak{n}$ prime to $p$, which implies the $q$-expansion principle for modular forms. That is, the $q$-expansion of $f \in H^{0}\left(T_{m, n}\left(\mathfrak{c}, \Gamma_{1}^{1}(\mathfrak{n})\right)_{/ W_{m}}, \mathcal{O}_{T_{m, n}}\right)$ at $\infty$ uniquely determines $f$. Following [H3, §4.1.7], We define

$$
V_{m, n}(\mathfrak{c}, \Gamma):=H^{0}\left(T_{m, n}(\mathfrak{c}, \Gamma)_{/ W_{m}}, \mathcal{O}_{T_{m, n}}\right)
$$




$$
\begin{aligned}
& V_{m, \infty}(\mathfrak{c}, \Gamma):=\bigcup_{n} V_{m, n}(\mathfrak{c}, \Gamma), \\
& V(\mathfrak{c}, \Gamma):={\underset{m}{\lim }}_{m} V_{m, \infty}(\mathfrak{c}, \Gamma),
\end{aligned}
$$

and

$$
\mathcal{V}(\mathfrak{c}, \Gamma):=\underset{m}{\lim } V_{m, \infty}(\mathfrak{c}, \Gamma) .
$$

Here the projective limit is with respect to the natural isomorphisms $V_{m+1, \infty} / p^{m} V_{m+1, \infty} \simeq V_{m, \infty}$ for all $m \in \mathbb{Z}_{>0}$ [H3], and the direct limit is with respect to the morphisms induced by multiplication by $p$. The space $V(\mathfrak{c}, \Gamma)$ is the space of $p$-adic $\mathfrak{c}$-Hilbert modular forms of level $\Gamma$. We put $\Lambda=$ $W\left[\left|1+p \mathbb{Z}_{p}\right|\right] \simeq W[|T|]$. Then $V(\mathfrak{c}, \Gamma)$ is a $W\left[\left|\mathbb{Z}_{p}^{\times}\right|\right]$-module and in particular, a $\Lambda$-module.

Let $\theta:\left(\mathcal{O} \otimes \mathbb{Z}_{p}\right)^{\times} \longrightarrow W^{\times}$be a character of finite order. We say that a form is of parallel weight $k \in \mathbb{Z}_{p}$ and character $\theta$ if for any $\alpha \in\left(\mathcal{O} \otimes \mathbb{Z}_{p}\right)^{\times}$, we have

$$
\alpha^{*} f\left(A, \lambda, \eta, \iota_{p^{\infty}}\right):=f\left(A, \lambda, \eta, \iota_{p^{\infty}} \circ \alpha^{-1}\right)=\theta(\alpha)\langle N(\alpha)\rangle^{k} f
$$

where $N:\left(\mathcal{O} \otimes \mathbb{Z}_{p}\right)^{\times} \longrightarrow \mathbb{Z}_{p}^{\times}$is the norm map and \langle\rangle$: \mathbb{Z}_{p}^{\times} \longrightarrow 1+p \mathbb{Z}_{p}$ is the projection map. We denote by $\mathcal{V}(\mathfrak{c}, \Gamma)[k, \theta]$ the space of $p$-adic modular forms of level $\Gamma$, weight $k$, and character $\theta$. Similarly, we denote by $H^{0}\left(T_{m, n}(\mathfrak{c}, \Gamma)_{/ W_{m}}, \mathcal{O}_{T_{m, n}}\right)[k]$ the subspace of $H^{0}\left(T_{m, n}(\mathfrak{c}, \Gamma)_{/ W_{m}}, \mathcal{O}_{T_{m, n}}\right)$ consisting of all elements $f$ satisfying

$$
\alpha^{*} f\left(A, \lambda, \eta, \iota_{p^{n}}\right):=f\left(A, \lambda, \eta, \iota_{p^{n}} \circ \alpha^{-1}\right)=\langle N(\alpha)\rangle^{k} f
$$

We denote by $\mathcal{V}(\mathfrak{c}, \Gamma)[k, \theta]($ resp. $\mathcal{V}(\mathfrak{c}, \Gamma)[k]$ when $\theta=\mathbf{1})$ the space consisting of elements $v \in$ $\mathcal{V}(\mathfrak{c}, \Gamma)$ such that $\alpha \cdot v=\theta(\alpha)\langle N(\alpha)\rangle^{k} v\left(\right.$ resp. $\left.\alpha \cdot v=\langle N(\alpha)\rangle^{k} v\right)$ for all $\alpha \in\left(\mathcal{O} \otimes \mathbb{Z}_{p}\right)^{\times}$. Similarly, for a Hecke character $\chi$ modulo $\mathfrak{n} p$, we denote by $\mathcal{V}(\mathfrak{c}, \Gamma)[\chi]$ the subspace of $\mathcal{V}(\mathfrak{c}, \Gamma)$ on which the group $\Gamma_{0}(\mathfrak{n} p)$ acts via $\chi$

One can define the Hecke action on $V(\mathfrak{c}, \Gamma)$ (see $[\mathrm{H} 3, \S 4.1 .10]$ for the definition). Recall that $e$ and $e^{\circ}$ are the idempotent elements attached to $U(p)$ and $T(p)$, respectively. We write $\mathcal{V}^{\text {ord }}(\mathfrak{c}, \Gamma)=$ 
$e \cdot \mathcal{V}(\mathfrak{c}, \Gamma)$. Let $V^{\text {ord }}(\mathfrak{c}, \Gamma)=\operatorname{Hom}\left(\mathcal{V}^{\text {ord }}(\mathfrak{c}, \Gamma), \mathbb{Q}_{p} / \mathbb{Z}_{p}\right)$ be the Pontryagin dual of $\mathcal{V}^{\text {ord }}(\mathfrak{c}, \Gamma)$, and let $V^{\text {ord }}(\mathfrak{c}, \Gamma, \chi)$ be the Pontryagin dual of $\mathcal{V}^{\text {ord }}(\mathfrak{c}, \Gamma)[\chi]$ for Hecke characters $\chi$ modulo $\mathfrak{n} p$.

The following theorem is called the vertical control theorem in [H3]. It tells us how to obtain the space of modular forms of weight $k$ and level $\Gamma$ from the space of $p$-adic modular forms.

Theorem 6.5.1. Let $\mathfrak{n}$ be an integral ideal of $F$ prime to $p$ with $\mathfrak{n} \cap \mathbb{Z}=(N)$, and let other notation be as above. Suppose that $p$ prime to $\phi(N) N d_{F} \mathfrak{c}$. If $k \in \mathbb{Z}_{\geq 2}$, then

1. For $r \in \mathbb{Z}_{>0}$, we have $e H^{0}\left(\mathfrak{S}\left(\mathfrak{c}, \Gamma_{1}^{r}\right), \underline{\omega}^{k}\right) \otimes \mathbb{Q}_{p} / \mathbb{Z}_{p} \simeq e H^{0}\left(\mathfrak{M}\left(\mathfrak{c}, \Gamma_{1}^{r}\right), \underline{\omega}^{k}\right) \otimes \mathbb{Q}_{p} / \mathbb{Z}_{p}$. Moreover, we have $e H^{0}\left(S(\mathfrak{c}, \Gamma), \underline{\omega}^{k}\right) \otimes \mathbb{Q}_{p} / \mathbb{Z}_{p} \simeq e^{\circ} H^{0}\left(M(\mathfrak{c}, \Gamma), \underline{\omega}^{k}\right) \otimes \mathbb{Q}_{p} / \mathbb{Z}_{p}$

2. If $k \geq 3$, we have $\mathcal{V}^{\text {ord }}(\mathfrak{c}, \Gamma)[k] \simeq e^{\circ} G_{k}(\mathfrak{c}, \Gamma ; W) \otimes \mathbb{Q}_{p} / \mathbb{Z}_{p}$.

3. If $k \geq 3$, we have $V^{\text {ord }}(\mathfrak{c}, \Gamma) \otimes_{\Lambda, k} W \simeq \operatorname{Hom}_{W}\left(e^{\circ} G_{k}(\mathfrak{c}, \Gamma ; W), W\right)$.

4. The space $V^{\text {ord }}(\mathfrak{c}, \Gamma)$ is a free $\Lambda$-module of finite rank.

Proof. We follow the argument of [H3, Theorem 4.10].

1. Suppose that $r>0$. We write $S=\mathfrak{S}\left(\mathfrak{c}, \Gamma_{1}^{r}\right)$ and $M=\mathfrak{M}\left(\mathfrak{c}, \Gamma_{1}^{r}\right)$ for simplicity. Since $S$ is an open subscheme of $M$, we have an embedding

$$
\epsilon_{m}: e H^{0}\left(M_{/ W}, \underline{\omega}^{k}\right) \otimes W_{m} \hookrightarrow e H^{0}\left(S_{/ W}, \underline{\omega}^{k}\right) \otimes W_{m}
$$

for $m \in \mathbb{Z}_{>0}$. We claim that $\epsilon_{m}$ is an isomorphism. Given any $\bar{f} \in e H^{0}\left(S_{/ W}, \underline{\omega}^{k}\right) \otimes W_{m}$, let $f \in e H^{0}\left(S_{/ W}, \underline{\omega}^{k}\right)$ be such that $f \equiv \bar{f} \bmod p^{m}$. Since $S=M\left[\frac{1}{E}\right]$, we have $H^{0}\left(S / W, \underline{\omega}^{k}\right)=$ $\lim _{n} H^{0}\left(M_{/ W}, \underline{\omega}^{k+n a(p-1)}\right) / E^{n}[H 3, \S 3.3 .2]$, where $E \in H^{0}\left(M_{/ W}, \underline{\omega}^{a(p-1)}\right)$ was defined in Section 6.4. Thus $E^{m} f$ belongs to $H^{0}\left(M_{/ W}, \underline{\omega}^{k+m a(p-1)}\right)$ for some $m \in \mathbb{Z}_{>0}$, and we have $e\left(E^{m} f\right) \in$ $e H^{0}\left(M_{/ W}, \underline{\omega}^{k+m a(p-1)}\right)$. Moreover, we have

$$
e\left(E^{m} f\right) \equiv E^{m}(e f)=E^{m} f \bmod p^{m}
$$


Let $K$ be the quotient field of $W$. In Section 6.2, we saw that $G_{k}^{\text {ord }}(\mathfrak{c}, \Gamma ; W)=e$. $H^{0}\left(M(\mathfrak{c}, \Gamma)_{/ W}, \underline{\omega}_{/ W}^{k}\right)$. Since the dimension of $G_{k}^{\text {ord }}\left(\mathfrak{c}, \Gamma_{1}^{r} ; K\right)$ is bounded independent of $k$ for $k \geq 2$ (see the proof of $\left[H 3\right.$, Theorem 4.9]), we have an isomorphism $e H^{0}\left(M, \underline{\omega}^{k}\right) \otimes$ $K \simeq e H^{0}\left(M, \underline{\omega}^{k+m a(p-1)}\right) \otimes K$ induced by multiplying by $E$. Therefore, there exists $g \in$ $e H^{0}\left(M_{/ W}, \underline{\omega}^{k}\right)$ and $l \in \mathbb{Z}_{\geq 0}$ such that $p^{l} \cdot e\left(E^{m} f\right)=E^{m} g$. Furthermore, we have

$$
p^{l} e\left(E^{m} f\right) \equiv p^{l} E^{m} f \equiv E^{m} g \bmod p^{l+m} .
$$

Since $E^{m} \equiv 1 \bmod p$ (see 6.1), we have $p^{l} f \equiv g \bmod p^{l+m}$, and hence, $g \in p^{l} e H^{0}\left(M_{/ W}, \underline{\omega}^{k}\right)$. We know that $f \equiv p^{-l} g \bmod p^{m}$. Thus $\epsilon_{m}$ is an isomorphism for all $m \in \mathbb{Z}_{>0}$. Since injective limit is an exact functor, we obtain the first isomorphism. By the same argument, we have the second isomorphism as well.

2. Recall that from the discussion in Section 6.2 , we have shown that $G_{k}\left(\mathfrak{c}, \Gamma_{1}(\mathfrak{n}) ; W\right)$ is a direct summand of $G_{k}\left(\mathfrak{c}, \Gamma_{1}^{1}(\mathfrak{n}) ; W\right)$ for $k \in \mathbb{Z}$. Also, it is known that $G_{k}\left(\mathfrak{c}, \Gamma_{1}^{1}(\mathfrak{n}) ; W\right)$ is a direct summand of $G_{k}(\mathfrak{c}, \Gamma(N) ; W)$ (see [H3, p.124]). Therefore, to prove the second and the third assertions, it suffices to prove the assertion for $\mathcal{V}^{\text {ord }}(\mathfrak{c}, \Gamma(N))$ and $V^{\text {ord }}(\mathfrak{c}, \Gamma(N))$. For simplicity, we write $\Gamma=\Gamma(N), \Gamma_{1}^{r}=\Gamma(N) \cap \Gamma_{1}\left(p^{r}\right), \Gamma_{0}^{r}=\Gamma(N) \cap \Gamma_{0}\left(p^{r}\right), \mathcal{V}=\mathcal{V}(\mathfrak{c}, \Gamma)$, and $V=V(\mathfrak{c}, \Gamma)$.

For $k \geq 3$, we have

$$
\begin{aligned}
& e \mathcal{V}[k]=\underset{m}{\lim } \underset{n}{\lim } e H^{0}\left(T_{m, n}, \mathcal{O}_{T_{m, n}}\right)[k]=\underset{m}{\lim } e H^{0}\left(T_{m, 1}, \mathcal{O}_{T_{m, 1}}\right)[k]=e H^{0}\left(S\left(\mathfrak{c}, \Gamma_{0}^{1}\right), \underline{\omega}^{k} \otimes \mathbb{Q}_{p} / \mathbb{Z}_{p}\right) \\
& ={ }^{(1)} e H^{0}\left(S(\mathfrak{c}, \Gamma), \underline{\omega}^{k} \otimes \mathbb{Q}_{p} / \mathbb{Z}_{p}\right)={ }^{(2)} e H^{0}\left(S, \underline{\omega}^{k}\right) \otimes \mathbb{Q}_{p} / \mathbb{Z}_{p} .
\end{aligned}
$$

Note that the first equality follows from the fact that the Hecke operator $T(p)$ sends each modular form of level $\Gamma_{1}\left(p^{r}\right)$ to a modular form of level $\Gamma_{1}\left(p^{r-1}\right)$ for all $r \geq 2$. The equality (1) is obtained by the fact that every $p$-ordinary modular form of level $\Gamma_{0}^{1}$ and weight $k \geq 3$ is 
old at $p$, and the equality (2) follows from Corollary 6.4.2. Then the assertion follows from the first assertion.

3. Since $V^{\text {ord }}$ is the Pontryagin dual of $\mathcal{V}^{\text {ord }}$, we have

$$
\begin{aligned}
V^{\text {ord }} \otimes_{\Lambda, k} W & =V^{\text {ord }} /\left(T-u^{k}+1\right) V^{\text {ord }}=\operatorname{Hom}\left(\mathcal{V}^{\text {ord }}[k], \mathbb{Q}_{p} / \mathbb{Z}_{p}\right) \\
& \simeq \operatorname{Hom}\left(e H^{0}\left(S, \underline{\omega}^{k}\right) \otimes \mathbb{Q}_{p} / \mathbb{Z}_{p}, \mathbb{Q}_{p} / \mathbb{Z}_{p}\right) \simeq \operatorname{Hom}\left(e^{\circ} G_{k}(\mathfrak{c}, \Gamma ; W), W\right) .
\end{aligned}
$$

Note that the first isomorphism follows from the second assertion and the second isomorphism follows from the first assertion.

4. Since the third assertion holds for all $k \geq 3$ and since $G_{k}(\mathfrak{c}, \Gamma ; W)$ is a free $W$-module of finite rank, the last assertion follows from the following well-known lemma.

Lemma 6.5.2. Let $M$ be a finitely generated torsion-free $\Lambda$-module. If $M / P$ is free for infinitely many height 1 prime ideals of $\Lambda$, then $M$ is free $\Lambda$-module of finite rank.

For a proof, see [H2, Chapter 7].

Recall that $\mu_{p^{\infty}}$ is the group of all $p$-power roots of unity. Also, recall that $\rho=\rho_{\zeta}$ is the character associated with the $p^{r-1}$ th root of unity $\zeta$ which is defined in Section 4.1. The next lemma is a key result to prove the control theorem (Corollary 6.5.5).

Lemma 6.5.3. Let $\chi$ be a narrow ray class character modulo $\mathfrak{n} p$. Assume that $W$ contains the values of $\chi$ and $\mu_{p^{\infty}}$, and suppose that the assumption in Theorem 6.5.1 holds. If $k \geq 2$ and $\zeta \in \mu_{p^{\infty}}$, then we have

$$
V^{\text {ord }}\left(\mathfrak{c}, \Gamma_{1}(\mathfrak{n})\right)^{(\chi)} \otimes_{W\left[\left|\mathbb{Z}_{p}^{\times}\right|\right], k, \rho} W \simeq \operatorname{Hom}_{W}\left(G_{k}^{\text {ord }}\left(\mathfrak{c}, \mathfrak{n} p^{r}, \chi \omega^{-k} \rho ; W\right), W\right)
$$

Proof. We follow the argument as in [Hsieh, Corollary 4.23]. For simplicity, we write $V^{\text {ord }}\left(\mathfrak{c}, \Gamma_{1}(\mathfrak{n}), \chi\right)$ as $V^{\text {ord }, \chi}$ and write $\mathcal{V}^{\text {ord }}\left(\mathfrak{c}, \Gamma_{1}(\mathfrak{n})\right)$ as $\mathcal{V}^{\text {ord }}$. Let $K$ be the quotient field of $W$. Since 
the conductor of the character $\chi \omega^{-k} \rho$ is $\mathfrak{n} p^{r}$, we have

$$
\mathcal{V}^{\text {ord }}\left[k, \chi \omega^{-k} \rho\right]=\underset{m}{\lim } \underset{n}{\lim } e H^{0}\left(T_{m, n}, \mathcal{O}_{T_{m, n}}\right)\left[k, \chi \omega^{-k} \rho\right]=\underset{m}{\lim } e H^{0}\left(S\left(\mathfrak{c}, \Gamma_{1}\left(\mathfrak{n} p^{r}\right), \underline{\omega}^{k}\right)^{\left(\chi \omega^{-k} \rho\right)},\right.
$$

where the last term is the subspace of $e H^{0}\left(S\left(\mathfrak{c}, \Gamma_{1}\left(\mathfrak{n} p^{r}\right), \underline{\omega}^{k}\right)\right.$ on which the group $\Gamma_{0}\left(\mathfrak{n} p^{r}\right)$ acts via $\chi \omega^{-k} \rho$. Let $G_{m}=e H^{0}\left(S\left(\mathfrak{c}, \Gamma_{1}\left(\mathfrak{n} p^{r}\right), \underline{\omega}^{k}\right)^{\left(\chi \omega^{-k} \rho\right)}\right.$, and let $G=G_{k}^{\text {ord }}\left(\mathfrak{n} p^{r}, \chi \omega^{-k} \rho ; W\right)$. Let $C_{m}$ be the cokernel of the embedding $G \otimes W_{m} \hookrightarrow G_{m}$ that exists by Theorem 6.5.1(1). Taking the injective limit, we obtain a short exact sequence

$$
0 \longrightarrow G \otimes \mathbb{Q}_{p} / \mathbb{Z}_{p} \longrightarrow \underline{\lim } G_{m} \longrightarrow \lim _{\longrightarrow} C_{m} \longrightarrow 0
$$

We set $C=\underline{\lim } C_{m}$. Taking the Pontryagin dual, we obtain

$$
0 \longrightarrow C^{*} \longrightarrow V^{\text {ord }, \chi} /\left(T-\rho(u) u^{k}+1\right) V^{\text {ord }, \chi} \longrightarrow \operatorname{Hom}_{W}(M, W) \longrightarrow 0
$$

We claim that $C^{*}=0$. Since $V^{\text {ord }}$ is free $\Lambda$-module of finite rank by Theorem 6.4.1 (4), $V^{\text {ord }}[\chi]$ is also free $\Lambda$-module of finite rank, and hence, $C^{*}$ is a torsion-free $W$-module. To show $C^{*}=0$, it suffices to show that $C^{*}$ is a torsion $W$-module. Indeed, we will show that $\left|\left(\mathbb{Z} / p^{r} \mathbb{Z}\right)^{\times}\right| G_{m} \subset G \otimes W_{m}$ for all $m \in \mathbb{Z}_{>0}$. Given any $f_{m} \in G_{m}$, by Corollary 6.4 .2 , there exists $f \in G$ such that $f \equiv f_{m} \bmod p^{m}$. Let $e_{\rho}=\sum_{\gamma \in\left(\mathbb{Z} / p^{r} \mathbb{Z}\right)^{\times}} \rho(\gamma) \cdot \gamma^{-1}$. Since $f \in G$, we have $\gamma \cdot f=\rho(\gamma) f$ for all $\gamma \in\left(\mathbb{Z} / p^{r} \mathbb{Z}\right)^{\times}$, and hence, we have

$$
\left|\left(\mathbb{Z} / p^{r} \mathbb{Z}\right)^{\times}\right| f_{m} \equiv\left|\left(\mathbb{Z} / p^{r} \mathbb{Z}\right)^{\times}\right| f=e_{\rho} f \in M \otimes W_{m} .
$$

Next, we define families of $p$-adic modular forms following [H3, §3.3.4]. For $\mu \in t_{\lambda}^{+} \cup\{0\}$, let $c_{\lambda}(\mu): \mathcal{V}^{\text {ord }}\left(t_{\lambda} \mathfrak{D}, \Gamma_{1}(\mathfrak{n})\right) \longrightarrow \mathbb{Q}_{p} / \mathbb{Z}_{p}$ be the linear map associating to $f$ its $e^{2 \pi i \operatorname{tr}(\mu z)}$-coefficient. Then $c_{\lambda}(\mu) \in V^{\text {ord }}$ for all $\mu$. Let $\chi$ be a narrow ray class character modulo $\mathfrak{n} p$, and let

$$
G^{\text {ord }}(\mathfrak{n}, \chi ; \Lambda)=\bigoplus_{\lambda=1}^{h_{F}^{+}} \operatorname{Hom}_{\Lambda}\left(V^{\text {ord }}\left(t_{\lambda} \mathfrak{D}, \Gamma_{1}(\mathfrak{n}), \chi\right), \Lambda\right) .
$$


To each $\mathcal{F} \in G^{\text {ord }}(\mathfrak{n}, \chi ; \Lambda)$, we associate its Fourier coefficients

$$
\left\{\begin{array}{cc}
C(\mathfrak{a}, \mathcal{F}) & \text { for all nonzero integral ideals } \mathfrak{a} \text { of } \mathcal{O}_{F} \\
C_{\lambda}(0, \mathcal{F}) & \lambda=1, \ldots, h_{F}^{+}
\end{array}\right\} .
$$

Here for an integral ideal $\mathfrak{a}$, we have $C(\mathfrak{a}, \mathcal{F})=N\left(t_{\lambda} \mathfrak{D}\right)^{-k / 2} \cdot \mathcal{F}\left(c_{\lambda}(\mu)\right)$ form some $\mu \in t_{\lambda} \mathfrak{D}$ satisfying $\mathfrak{a}=(\mu)\left(t_{\lambda} \mathfrak{D}\right)^{-1}$, and similarly, we have $C_{\lambda}(0, \mathcal{F})=N\left(t_{\lambda} \mathfrak{D}\right)^{-k / 2} \cdot \mathcal{F}\left(c_{\lambda}(0)\right)$. The following lemma shows that $G^{\text {ord }}(\mathfrak{n}, \chi ; \Lambda)$ and $M^{\text {ord }}(\mathfrak{n}, \chi ; \Lambda)$ are isomorphic. Recall that the space $M^{\text {ord }}(\mathfrak{n}, \chi ; \Lambda)$ of $\Lambda$-adic modular forms is defined in Section 4 .

Theorem 6.5.4. Let the notation be as above. Suppose that the assumption in Lemma 6.5.3 holds. Then we have an isomorphism of $\Lambda$-modules

$$
G^{\text {ord }}(\mathfrak{n}, \chi ; \Lambda) \simeq M^{\text {ord }}(\mathfrak{n}, \chi ; \Lambda)
$$

In particular, $M^{\text {ord }}(\mathfrak{n}, \chi ; \Lambda)$ is a free $\Lambda$-module of finite rank.

Proof. We follow the argument as in [Hsieh, Theorem 4.25]. By the $q$-expansion principle, we have a natural embedding

$$
G^{\text {ord }}(\mathfrak{n}, \chi ; \Lambda) \hookrightarrow M^{\text {ord }}(\mathfrak{n}, \chi ; \Lambda)
$$

Recall that we denote by $Q(\Lambda)$ the fraction field of $\Lambda$. It follows from the proof of [Wil2, Theorem 1.2.2] that the dimension of $M^{\prime \text { ord }}(\mathfrak{n}, \chi ; \Lambda) \otimes_{\Lambda} Q(\Lambda)$ over $Q(\Lambda)$ is less than or equal to $\operatorname{rank}_{W} M_{k}^{\text {ord }}\left(\mathfrak{n} p, \chi \omega^{-k}, W\right)$. By Lemma 6.5 .3 , we know that $\operatorname{rank}_{W} M_{k}^{\text {ord }}\left(\mathfrak{n} p, \chi \omega^{-k}, W\right)=$ $\operatorname{dim}_{Q(\Lambda)} G^{\text {ord }}(\mathfrak{n}, \chi ; \Lambda) \otimes_{\Lambda} Q(\Lambda)$. Therefore, we obtain an isomorphism

$$
G^{\text {ord }}(\mathfrak{n}, \chi ; \Lambda) \otimes_{\Lambda} Q(\Lambda) \simeq M^{\prime \text { ord }}(\mathfrak{n}, \chi ; \Lambda) \otimes_{\Lambda} Q(\Lambda)
$$

Let $\left\{\mathcal{F}_{1}, \ldots, \mathcal{F}_{s}\right\} \subset M^{\text {ord }}(\mathfrak{n}, \chi ; \Lambda)$ be a basis of $M^{\prime \text { ord }}(\mathfrak{n}, \chi ; \Lambda) \otimes_{\Lambda} Q(\Lambda)$. Thus, for every element $\mathcal{F} \in M^{\text {ord }}(\mathfrak{n}, \chi ; \Lambda)$, one has $\mathcal{F}=\sum_{i=1}^{s} x_{i} \mathcal{F}_{i}$ for some $x_{i} \in Q(\Lambda)$. For any $s$ positive integral ideals 
$\mathfrak{a}_{1}, \ldots, \mathfrak{a}_{s}$, we have an equation of matrices

$$
A X=B
$$

for $A=\left(C\left(\mathfrak{a}_{i}, \mathcal{F}_{j}\right)\right), X=\left(x_{1}, \ldots, x_{s}\right)^{t}$, and $B=\left(C\left(\mathfrak{a}_{i}, \mathcal{F}\right)^{t}\right)$. Here $A^{t}$ is the transpose of a matrix $A$. Since $\left\{\mathcal{F}_{1}, \ldots, \mathcal{F}_{s}\right\} \subset M^{\text {ord }}(\mathfrak{n}, \chi ; \Lambda)$ is a basis of $M^{\text {ord }}(\mathfrak{n}, \chi ; \Lambda) \otimes_{\Lambda} Q(\Lambda)$, one can pick integral ideals $\mathfrak{a}_{1}, \ldots, \mathfrak{a}_{s}$ of $F$ such that $a=\operatorname{det} A \neq 0 \in \Lambda$. By multiplying the adjoint matrix of $A$ on both sides, we see that $a \mathcal{F} \in \Lambda \cdot \mathcal{F}_{1}+\cdots+\Lambda \cdot \mathcal{F}_{s}$. Therefore, we have $a M^{\prime \text { ord }}(\mathfrak{n}, \chi ; \Lambda) \subset \mathcal{F}_{1}+\cdots+\Lambda \cdot \mathcal{F}_{s}$. In particular, $M^{\prime \text { ord }}(\mathfrak{n}, \chi ; \Lambda)$ is a finitely generated $\Lambda$-module since $\Lambda$ is Noetherian. By the same argument as in [H2, p.210], we know that $M^{\text {ord }}(\mathfrak{n}, \chi ; \Lambda) /\left(T-u^{k}+1\right)$ is a free $W$-module of finite rank for almost all $k \in \mathbb{Z}_{\geq 2}$, and hence $M^{\prime \text { ord }}(\mathfrak{n}, \chi ; \Lambda)$ is a free $\Lambda$-module of finite rank by Lemma 6.5.2.

We set $N=M^{\prime \text { ord }}(\mathfrak{n}, \chi ; \Lambda) / G^{\text {ord }}(\mathfrak{n}, \chi ; \Lambda)$. Then $N$ is a torsion $\Lambda$-module. To prove the assertion, we will show that $N=0$ by showing that $N$ is a flat $\Lambda$-module. Let $\kappa$ be the residue field of $\Lambda$. Since $M^{\prime \text { ord }}(\mathfrak{n}, \chi ; \Lambda)$ is a free $\Lambda$-module, we obtain the exact sequence

$$
0 \longrightarrow \operatorname{Tor}^{1}(N, \kappa) \longrightarrow G^{\text {ord }}(\mathfrak{n}, \chi ; \Lambda) \otimes \kappa \stackrel{\iota}{\rightarrow} M^{\text {ord }}(\mathfrak{n}, \chi ; \Lambda) \otimes \kappa \longrightarrow N \otimes \kappa \longrightarrow 0
$$

By the $q$-expansion principle again, $\iota$ is injection, and hence, $\operatorname{Tor}^{1}(N, \kappa)=0$. It follows that $N$ is a flat $\Lambda$-module since $\Lambda$ is a local Noetherian ring.

Corollary 6.5.5 (Control theorem). Let the notation and the assumption be as in Lemma 6.5.3.

Then for each integer $k \geq 2$, we have an isomorphism

$$
M^{\text {ord }}(\mathfrak{n}, \chi ; \Lambda) /\left(T-\rho(u) u^{k-2}+1\right) \simeq M_{k}^{\text {ord }}\left(\mathfrak{n} p^{r}, \chi \omega^{2-k} \rho ; W\right)
$$

Moreover, $M^{\text {ord }}(\mathfrak{n}, \chi ; \Lambda)$ is free $\Lambda$-module of finite rank. 
Proof. We follow the argument in [H3, Theorem 3.8]. By Lemma 5.1.2, Lemma 6.5.3 and Theorem 6.5.4, we have

$$
\begin{aligned}
M^{\text {ord }}(\mathfrak{n}, \chi ; \Lambda) /\left(T-\rho(u) u^{k-2}+1\right) & \simeq M^{\text {ord }}\left(\mathfrak{n}, \chi \omega^{2} ; \Lambda\right) /\left(T-\rho(u) u^{k}+1\right) \\
& \simeq G^{\text {ord }}\left(\mathfrak{n}, \chi \omega^{2} ; \Lambda\right) /\left(T-\rho(u) u^{k}+1\right) \\
& \simeq\left(\bigoplus_{\lambda=1}^{h_{F}^{+}} \operatorname{Hom}_{\Lambda}\left(V^{\text {ord }}\left(t_{\lambda} \mathfrak{D}, \mathfrak{n}\right)\left[\chi \omega^{2}\right], \Lambda\right)\right) \otimes_{W\left[\left[\mathbb{Z}_{p}^{\times}\right], k, \rho\right.} W \\
& \simeq \bigoplus_{\lambda=1}^{h_{F}^{+}}\left(\operatorname{Hom}_{\Lambda}\left(V_{\Lambda}^{\text {ord }}\left(t_{\lambda} \mathfrak{D}, \mathfrak{n}\right)\left[\chi \omega^{2}\right], \Lambda\right) \otimes_{\left.W\left[\mid \mathbb{Z}_{p}^{\times}\right]\right], k, \rho} W\right) \\
& \simeq \bigoplus_{\lambda=1}^{h_{F}^{+}} \operatorname{Hom}_{W}\left(V_{\Lambda}^{\text {ord }}\left(t_{\lambda} \mathfrak{D}, \mathfrak{n}\right)\left[\chi \omega^{2}\right] \otimes_{\left.W\left[\mathbb{Z}_{p}^{\times}\right]\right], k, \rho} W, W\right) \\
& \simeq \bigoplus_{\lambda=1}^{h_{F}^{+}} \operatorname{Hom}_{W}\left(\operatorname{Hom}_{W}\left(G_{k}^{\text {ord }}\left(t_{\lambda} \mathfrak{D}, \mathfrak{n} p^{r}, \chi \omega^{2-k} \rho ; W\right), W\right), W\right) \\
& \simeq M_{k}^{\text {ord }}\left(\mathfrak{n} p^{r}, \chi \omega^{2-k} \rho ; W\right) .
\end{aligned}
$$

The second assertion is obtained by Lemma 6.5.2. 


\title{
CHAPTER 7
}

\author{
Main results
}

Let $p$ be an odd rational prime, and let $\mathfrak{n}$ be an integral ideal prime to $p$. We fix two primitive narrow ray class characters $\chi_{1}$ and $\chi_{2}$ of conductors $\mathfrak{n}_{1}$ and $\mathfrak{n}_{2}$, respectively. We assume that $\mathfrak{n}_{1} \mathfrak{n}_{2}=\mathfrak{n}$ or $\mathfrak{n} p$ and $\mathfrak{n}_{2}$ is prime to $p$. Let $\mathcal{O}_{\infty}$ be a complete valuation ring in $\mathbb{C}_{p}$ containing all $p$-power roots of unity and values of $\chi_{1}$ and $\chi_{2}$, and let $\Lambda_{\infty}=\mathcal{O}_{\infty}[|T|]$. For simplicity, we write $M_{\Lambda_{\infty}}=M^{\text {ord }}\left(K_{1}(\mathfrak{n}) ; \Lambda_{\infty}\right)$ and write $S_{\Lambda_{\infty}}$ in the same manner.

\subsection{Proof of Theorem 1.0.1: Part 1}

The first short exact sequence in (1.1) is constructed as follows. First of all, we have

$$
0 \longrightarrow S_{\Lambda_{\infty}} \longrightarrow M_{\Lambda_{\infty}} \stackrel{C_{0}}{\longrightarrow} e \cdot \Lambda_{\infty}\left[C_{\mathfrak{n} p}^{*}\right]
$$

Recall that the map $C_{0}$ is defined in Section 3.4. We claim that the homomorphism $C_{0}$ is surjective. Recall that in Chapter 1, we impose two hypotheses:

H1 $p$ does not divide $N_{F / \mathbb{Q}}(\mathfrak{n} \mathfrak{D}) \phi\left(N_{F / \mathbb{Q}}(\mathfrak{n})\right) h_{F}$,

H2 $\mathfrak{n}$ is prime to $\mathfrak{D}$ and does not divide 2 and 3 .

Lemma 7.1.1. Suppose that $\mathbf{H 1}$ and $\mathbf{H 2}$ hold. Then the sequence

$$
0 \longrightarrow S_{\Lambda_{\infty}} \longrightarrow M_{\Lambda_{\infty}} \stackrel{C_{0}}{\longrightarrow} e \cdot \Lambda_{\infty}\left[C_{\mathfrak{n} p}^{*}\right] \longrightarrow 0
$$

is exact. 
Proof. We follow the argument of [Hsieh, Theorem 4.26]. Since $p$ does not divide $N(\mathfrak{n}) \phi(N(\mathfrak{n})) h_{F}$, we have $M_{\Lambda_{\infty}}=\oplus_{\chi} M_{\Lambda_{\infty}}^{(\chi)}$ and $e \cdot \Lambda_{\infty}\left[C_{\mathfrak{n} p}^{*}\right]=\oplus_{\chi} e \cdot \Lambda_{\infty}\left[C_{\mathfrak{n} p}^{*}\right]^{(\chi)}$, where the direct sums run through all narrow ray class characters modulus $\mathfrak{n} p$. Here $M_{\Lambda_{\infty}}^{(\chi)}$ and $e \cdot \Lambda_{\infty}\left[C_{\mathfrak{n} p}^{*}\right]^{(\chi)}$ are respectively the $\chi^{-}$ eigenspaces of $M_{\Lambda_{\infty}}$ and of $e \cdot \Lambda_{\infty}\left[C_{\mathfrak{n} p}^{*}\right]$. To show that $C_{0}$ is surjective, it suffices to show that the induced map $C_{0}: M_{\Lambda_{\infty}}^{(\chi)} \longrightarrow e \cdot \Lambda_{\infty}\left[C_{\mathfrak{n} p}^{*}\right]^{(\chi)}$ is surjective.

We write $\chi=\psi \omega^{l}$ for some $l \in \mathbb{Z}_{\geq 0}$ and for some character $\psi$ of modulus $\mathfrak{n}$. Let $\mathfrak{P}$ be the maximal ideal of $\mathcal{O}_{\infty}$, and let $\mathbb{F}=\mathcal{O}_{\infty} / \mathfrak{P}$ be its residue field. By Nakayama's lemma, it suffice to show that the map

$$
M_{\Lambda_{\infty}}^{(\chi)} /(\mathfrak{P}, T) \stackrel{C_{0}}{\longrightarrow} e \cdot \Lambda_{\infty}\left[C_{\mathfrak{n} p}^{*}\right]^{(\chi)} /(\mathfrak{P}, T)
$$

is surjective. By Lemma 6.4.1 and Corollary 6.5.5, for $k$ with $2-k+l \equiv 0 \bmod (p-1)$ (or equivalently, $\left.\chi \omega^{2-k}\right|_{(\mathbb{Z} / p \mathbb{Z})^{\times}}$is a trivial character $)$, we have

$$
M_{\Lambda_{\infty}}^{(\chi)} /(\mathfrak{P}, T) \simeq M_{k}^{\text {ord }}\left(\mathfrak{n}, \psi ; \mathcal{O}_{\infty}\right) \otimes_{\mathcal{O}_{\infty}} \mathbb{F} \simeq e H^{0}\left(S_{\mathcal{O}_{\infty}}^{*}, \pi_{*}\left(\underline{\omega}^{k}\right)\right)^{(\psi)} \otimes_{\mathcal{O}_{\infty}} \mathbb{F}
$$

Moreover, we have

$$
e \cdot \Lambda_{\infty}\left[C_{\mathfrak{n} p}^{*}\right]^{(\chi)} /(\mathfrak{P}, T) \simeq e \cdot \mathcal{O}_{\infty}\left[C_{\mathfrak{n}}^{*}\right]^{(\psi)} \otimes_{\mathcal{O}_{\infty}} \mathbb{F}=e H^{0}\left(\partial S_{/ \mathcal{O}_{\infty}}^{*}, \pi_{*}\left(\underline{\omega}^{k}\right)\right)^{(\psi)} \otimes_{\mathcal{O}_{\infty}} \mathbb{F}
$$

Since $S^{*}$ is affine and $\pi_{*}\left(\underline{\omega}^{k}\right)$ is an invertible sheaf, the map

$$
C_{0}: e H^{0}\left(S_{/ \mathcal{O}_{\infty}}^{*}, \pi_{*}\left(\underline{\omega}^{k}\right)\right)^{(\psi)} \otimes_{\mathcal{O}_{\infty}} \mathbb{F} \longrightarrow e H^{0}\left(\partial S_{/ \mathcal{O}_{\infty}}, \pi_{*}\left(\underline{\omega}^{k}\right)\right)^{(\psi)} \otimes_{\mathcal{O}_{\infty}} \mathbb{F}
$$

it is surjective. This shows that $C_{0}$ is surjective on each $\chi$-component.

Let $\Lambda=\mathbb{Z}_{p}\left[\chi_{1}, \chi_{2}\right][|T|]$. It is known that $\Lambda_{\infty}$ is a faithfully flat $\Lambda$-module [Ohta3, Lemma 2.1.1]. Since the short exact sequence in Lemma 7.1 .1 can be obtained by tensoring with $\Lambda_{\infty}$ over $\Lambda$, we obtain a short exact sequence of $\Lambda$-modules

$$
0 \longrightarrow S^{\text {ord }}\left(K_{1}(\mathfrak{n}) ; \Lambda\right) \longrightarrow M^{\text {ord }}\left(K_{1}(\mathfrak{n}) ; \Lambda\right) \stackrel{C_{0}}{\longrightarrow} e \cdot \Lambda\left[C_{\mathfrak{n} p}^{*}\right] \longrightarrow 0
$$


Recall that $\mathfrak{M}=\mathfrak{M}\left(\chi_{1}, \chi_{2}\right)$ is the maximal ideal of $\mathcal{H}^{\text {ord }}$ defined in Definition 5.2.4. Let $M_{\Lambda}=$ $M^{\text {ord }}\left(K_{1}(\mathfrak{n}) ; \Lambda\right)_{\mathfrak{M}}\left(\operatorname{resp} . S_{\Lambda}=S^{\text {ord }}\left(K_{1}(\mathfrak{n}) ; \Lambda\right)_{\mathfrak{M}}\right.$ and $\left.e \cdot \Lambda\left[C_{\mathfrak{n} p}^{*}\right]_{\mathfrak{M}}\right)$ be the localization of $M^{\text {ord }}\left(K_{1}(\mathfrak{n}) ; \Lambda\right)$ (resp. $S^{\operatorname{ord}}\left(K_{1}(\mathfrak{n}) ; \Lambda\right)$ and $\left.e \cdot \Lambda_{\infty}\left[C_{\mathfrak{n} p}^{*}\right]\right)$ at $\mathfrak{M}$.

Lemma 7.1.2. Assume that $\mathbf{H 1}-\mathbf{H 2}$ hold. If $\left(\chi_{1}, \chi_{2}\right) \neq\left(\omega^{-2}, \mathbf{1}\right)$ and $\chi_{1} \omega \chi_{2}^{-1}(\mathfrak{p}) \neq 1$ for some prime ideal $\mathfrak{p} \mid p$, then $e \cdot \Lambda\left[C_{\mathfrak{n} p}^{*}\right]_{\mathfrak{M}}$ is a free $\Lambda$-module of rank one. Moreover, the Hecke operator $T_{\mathfrak{p}}$ acts on $e \cdot \Lambda_{\infty}\left[C_{\mathfrak{n} p}^{*}\right]_{\mathfrak{M}}$ by multiplication by $\bar{\chi}_{1}(\mathfrak{p}) N(\mathfrak{p})+\chi_{2}(\mathfrak{p})$ for all prime ideals $\mathfrak{p}$ and $S(\mathfrak{q})$ acts on it by multiplication $\overline{\chi_{1}} \chi_{2}(\mathfrak{q})$ for all prime ideals $\mathfrak{q}$ not dividing $\mathfrak{n} p$.

Proof. We follow the argument in [Ohta4, Proposition 3.1.2]. Suppose the rank of $e \cdot \Lambda\left[C_{\mathfrak{n} p}^{*}\right]_{\mathfrak{M}}$ is greater or equal to two. Then $e \cdot \Lambda\left[C_{\mathfrak{n} p}^{*}\right]_{\mathfrak{M}} \otimes_{\Lambda} Q(\Lambda)$ is a $Q(\Lambda)$-vector space of dimension greater than one. Then there exists an Eisenstein series $\mathcal{E}(\theta, \psi) \in M_{\Lambda}$ with $\theta \psi=\chi_{1} \chi_{2}$ and $(\theta, \psi) \neq\left(\chi_{1}, \chi_{2}\right)$. By Proposition 5.2.5, we know that $\chi_{1} \omega \chi_{2}^{-1}(\mathfrak{p})=1$ for all prime ideals $\mathfrak{p} \mid p$, which contradicts to the assumption. Therefore, $e \cdot \Lambda\left[C_{\mathfrak{n} p}^{*}\right]_{\mathfrak{M}}$ is free of rank one. The second assertion follows from the fact that the map $C_{0}$ is Hecke equivariant.

Let $c_{\infty}$ be a basis of $e \cdot \Lambda\left[C_{\mathfrak{n} p}^{*}\right]_{\mathfrak{M}}$ over $\Lambda$. We obtain a short exact sequence of $\Lambda$-modules

$$
0 \longrightarrow S_{\Lambda} \longrightarrow M_{\Lambda} \stackrel{C_{0}}{\longrightarrow} \Lambda \cdot c_{\infty} \longrightarrow 0
$$

which is the first sequence in (1.1).

We set

$$
A\left(\chi_{1}, \chi_{2}\right):=\prod_{\substack{\mathfrak{q} \mid \mathfrak{n} \\ \mathfrak{q} \nmid \operatorname{cond}\left(\chi_{1} \chi_{2}^{-1}\right)}}\left(1-\chi_{1} \chi_{2}^{-1}(\mathfrak{q})(1+T)^{-s(\mathfrak{q})} N(\mathfrak{q})^{-2}\right) \widehat{G}_{\chi_{1} \chi_{2}^{-1}}(T),
$$

where $s(\mathfrak{q}) \in \mathbb{Z}_{p}$ is defined by $(5.1)$.

Theorem 7.1.3. Assume that $\mathbf{H 1}-\mathbf{H} 2$ hold. Then the congruence module attached to the short 
exact sequence

$$
0 \longrightarrow S_{\Lambda} \longrightarrow M_{\Lambda} \stackrel{C_{0}}{\longrightarrow} \Lambda \cdot c_{\infty} \longrightarrow 0
$$

is $\Lambda /\left(A\left(\chi_{1}, \chi_{2}\right)\right)$.

Proof. Let $K$ be a field extension of $\mathbb{Q}_{p}$. Since the space $M_{k}\left(K_{1}(\mathfrak{n} p) ; K\right)$ is a direct sum of the space of cusp forms and the space generated by Eisenstein series, we know that over $Q(\Lambda)$, the space of $\Lambda$-adic modular forms is a direct sum of the space of $\Lambda$-adic cusp forms and the space generated by $\Lambda$-adic Eisenstein series. In particular, we have

$$
M_{\Lambda} \otimes_{\Lambda} Q(\Lambda)=\left(S_{\Lambda} \otimes_{\Lambda} Q(\Lambda)\right) \oplus\left(\mathcal{E}_{\Lambda} \otimes_{\Lambda} Q(\Lambda)\right),
$$

where $\mathcal{E}_{\Lambda}$ is the $\Lambda$-module generated by $\mathcal{E}\left(\chi_{1}, \chi_{2}\right)$. Let

$$
s: \mathcal{E}_{\Lambda} \otimes_{\Lambda} Q(\Lambda) \simeq Q(\Lambda)=\Lambda \cdot c_{\infty} \otimes_{\Lambda} Q(\Lambda) \longrightarrow M_{\Lambda} \otimes_{\Lambda} Q(\Lambda)
$$

be the splitting map. We have

$$
s(Q(\Lambda)) \cap M_{\Lambda}=\mathcal{E}\left(\chi_{1}, \chi_{2}\right) \Lambda,
$$

and hence the congruence module is isomorphic to $\Lambda / C_{0}\left(\mathcal{E}\left(\chi_{1}, \chi_{2}\right)\right) \Lambda$. From Proposition 5.2.3, we see that the common factor of the constant terms of $\mathcal{E}\left(\chi_{1}, \chi_{2}\right)$ at different cusps is a unit in $\Lambda$ times $A\left(\chi_{1}, \chi_{2}\right)$, which implies that $C_{0}\left(\mathcal{E}\left(\chi_{1}, \chi_{2}\right)\right) \Lambda=A\left(\chi_{1}, \chi_{2}\right) \Lambda$.

\subsection{Proof of Theorem 1.0.2}

Let the notation and the assumption be as in Section 7.1. For simplicity, we write $\mathcal{H}=$ $\mathcal{H}^{\text {ord }}\left(\mathfrak{n}, \chi_{1} \chi_{2} ; \Lambda\right)_{\mathfrak{M}}$ and $h=h^{\text {ord }}\left(\mathfrak{n}, \chi_{1} \chi_{2} ; \Lambda\right)_{\mathfrak{M}}$. The goal of this section is to prove Theorem 1.0.2. The first step is to construct a nice basis of $M_{\Lambda}$. Since $S_{\Lambda}$ is a finitely generated free $\Lambda$-module, we 
may let $\left\{\mathcal{F}_{1}, \ldots, \mathcal{F}_{m}\right\}$ be a basis of $S_{\Lambda}$ over $\Lambda$. Since $\operatorname{Hom}_{\Lambda}\left(S_{\Lambda}, \Lambda\right) \simeq h[\mathrm{H} 1, \S 3]$, there exists a $\Lambda$-basis $\left\{h_{1}, \ldots, h_{m}\right\}$ such that

$$
C\left(1, h_{j} \cdot \mathcal{F}_{i}\right)= \begin{cases}1 & \text { if } i \neq j \\ 0 & \text { otherwise. }\end{cases}
$$

For each $i$, let $H_{i} \in \mathcal{H}$ map to $h_{i}$ via the natural projection $\mathcal{H} \rightarrow h$. Now take any element $\mathcal{F}^{\prime} \in M_{\Lambda}$ such that $C_{0}\left(\mathcal{F}^{\prime}\right)=c_{\infty}$. By Theorem 7.1.3, we know that $C_{0}\left(\mathcal{E}\left(\chi_{1}, \chi_{2}\right)\right)=u A\left(\chi_{1}, \chi_{2}\right) \cdot c_{\infty}$ for some unit $u \in \Lambda^{\times}$. Let $\mathcal{F}=u \cdot \mathcal{F}^{\prime}$, and let

$$
\mathcal{F}_{0}=\mathcal{F}-\sum_{i=1}^{m} C\left(1, H_{i} \cdot \mathcal{F}\right) \mathcal{F}_{i} \in M_{\Lambda}
$$

Then we have $C_{0}\left(\mathcal{F}_{0}\right)=C_{0}\left(u \mathcal{F}^{\prime}\right)=u \cdot c_{\infty}$. Since $\left(\chi_{1}, \chi_{2}\right)$ is not exceptional, the $\Lambda$-rank of $S_{\Lambda}$ is one less than the $\Lambda$-rank of $M_{\Lambda}$. Thus, it follows that the set $\left\{\mathcal{F}_{0}, \ldots, \mathcal{F}_{m}\right\}$ is a $\Lambda$-basis of $M_{\Lambda}$. Before we move to the second step, we make some observations on $\mathcal{F}_{0}$.

Proposition 7.2.1. Let the notation be as above.

1. We have $C_{0}\left(A\left(\chi_{1}, \chi_{2}\right) \mathcal{F}_{0}-\mathcal{E}\left(\chi_{1}, \chi_{2}\right)\right)=0$.

2. We can write $\mathcal{F}_{0}=\frac{\mathcal{E}\left(\chi_{1}, \chi_{2}\right)-\mathcal{F}_{S}}{A\left(\chi_{1}, \chi_{2}\right)}$ for some $\mathcal{F}_{S} \in S_{\Lambda}$.

3. Modulo $S_{\Lambda}, \mathcal{F}_{0}$ is a common eigenform whose eigenvalues are the same as those of $\mathcal{E}\left(\chi_{1}, \chi_{2}\right)$.

4. We have $C\left(1, H_{i} \cdot \mathcal{F}_{0}\right)=0$ for all $i=1, \ldots, m$.

Proof. (1) follows from the construction of $\mathcal{F}_{0}$. (2) and (3) follow from (1) and the exactness of (7.1). For (4), we have

$$
\begin{aligned}
C\left(1, H_{i} \cdot \mathcal{F}_{0}\right) & =C\left(1, H_{i} \cdot \mathcal{F}\right)-\sum_{j=1}^{m} C\left(1, H_{j} \cdot \mathcal{F}\right) C\left(1, H_{i} \cdot \mathcal{F}_{j}\right) \\
& =C\left(1, H_{i} \cdot \mathcal{F}\right)-C\left(1, H_{i} \cdot \mathcal{F}\right)=0
\end{aligned}
$$

for all $i=1, \ldots, m$. 
We now fix a cusp form $\mathcal{F}_{S}$ satisfying Proposition 7.2.1(2). Note that the cusp form $\mathcal{F}_{S}$ is congruent to the Eisenstein series $\mathcal{E}\left(\chi_{1}, \chi_{2}\right)$ modulo $A\left(\chi_{1}, \chi_{2}\right)$. We define the surjective $\Lambda$-algebra homomorphism in Theorem 1.0.2 as

$$
\Psi: h \longrightarrow \Lambda / A\left(\chi_{1}, \chi_{2}\right) ; T \mapsto C\left(1, T \cdot \mathcal{F}_{S}\right)
$$

Now we assume that $\chi_{2}=1$. To prove that $\Psi$ is an isomorphism on $h / I$, by Theorem 7.2.3, we shall see that it suffices to prove the following lemma. Recall that $I$ is the ideal of $h$ defined in Definition 5.2.4.

Lemma 7.2.2. Let the notation be as above. Then there exists an element $H \in \mathcal{H}$ such that $C(1, H$. $\left.\mathcal{F}_{0}\right) \in \Lambda$ is a unit.

Proof. Suppose that $C\left(1, H \cdot \mathcal{F}_{0}\right)$ is not a unit in $\Lambda$ for all $H \in \mathcal{H}$. Note that $\Lambda$ is a local ring with maximal ideal $m=(T, \varpi)$, where $\varpi$ is a uniformizer of $\mathbb{Z}_{p}\left[\chi_{1}\right]$. Also, note that $C\left(\mathfrak{a}, \mathcal{F}_{0}\right)=$ $C\left(1, T(\mathfrak{a}) \cdot \mathcal{F}_{0}\right) \in m$ for all integral $\mathfrak{a}$ of $\mathcal{O}_{F}$ since $\Lambda$ is a local ring and $C\left(1, T(\mathfrak{a}) \cdot \mathcal{F}_{0}\right)$ is not a unit. Moreover, since $C\left(1, \mathcal{E}\left(\chi_{1}, \mathbf{1}\right)\right)=1$, for $\mathfrak{p} \mid p$, we have $T(\mathfrak{p}) \cdot \mathcal{F}_{0}=\mathcal{F}_{0}+\mathcal{F}_{T \mathfrak{p}}$ for some $\mathcal{F}_{T_{\mathfrak{p}}} \in S_{\Lambda}$. Thus for all integral ideals $\mathfrak{a}$ and for all prime ideals $\mathfrak{p} \mid p$, we have

$$
C\left(1, T(\mathfrak{a}) T(\mathfrak{p}) \cdot \mathcal{F}_{0}\right)=C\left(\mathfrak{a}, T(\mathfrak{p}) \cdot \mathcal{F}_{0}\right)=C\left(\mathfrak{a}, \mathcal{F}_{0}\right)+C\left(\mathfrak{a}, \mathcal{F}_{T_{\mathfrak{p}}}\right)
$$

Therefore, we know that $C\left(\mathfrak{a}, \mathcal{F}_{T_{\mathfrak{p}}}\right) \in m$ for all integral ideals $\mathfrak{a}$ and prime ideals $\mathfrak{p} \mid p$.

Let $f_{2}=v_{2,1}\left(\mathcal{F}_{0}\right) \in M_{2}\left(\mathfrak{n} p, \chi_{1} ; \mathbb{Z}_{p}\left[\chi_{1}\right]\right)^{\text {ord }}$, and let $f_{0}=C\left(0, v_{2,1}\left(\mathcal{F}_{0}\right)\right) \in M_{0}\left(\mathfrak{n} p ; \mathbb{Z}_{p}\left[\chi_{1}\right]\right)$. Then by the construction of $\mathcal{F}_{0}$, we know that

$$
f_{2} \equiv f_{0} \bmod \varpi \text { and }\langle\mathfrak{q}\rangle f_{2} \equiv \chi_{1}(\mathfrak{q}) f_{2} \bmod \varpi
$$


for all prime ideals $\mathfrak{q}$ coprime to $\mathfrak{n} p$. Thus for any prime ideal $\mathfrak{q}$ coprime to $\mathfrak{n} p$, we have

$$
\begin{aligned}
\left(\chi_{1}(\mathfrak{q})-1\right) f_{0} & =\chi_{1}(\mathfrak{q}) f_{0}-f_{0} \\
& \equiv \chi_{1}(\mathfrak{q}) f_{2}-f_{0} \bmod \varpi \\
& \equiv \chi_{1}(\mathfrak{q}) f_{2}-f_{0} \bmod \varpi \\
& \equiv\langle\mathfrak{q}\rangle\left(f_{2}-f_{0}\right) \equiv 0 \bmod \varpi
\end{aligned}
$$

Furthermore, since $\chi_{1}$ is not a trivial character, we can choose a prime ideal $\mathfrak{q}$ such that $\chi_{1}(\mathfrak{q})-1$ is not congruent to zero modulo $\varpi$. Hence, there exists an element $H \in \mathcal{H}$ such that $C\left(1, H \cdot \mathcal{F}_{0}\right) \in \Lambda^{\times}$.

Let

$$
M_{\Lambda}^{\prime}=\left\{\mathcal{G} \in M_{\Lambda} \otimes_{\Lambda} Q(\Lambda) \mid C(\mathfrak{a}, \mathcal{G}) \in \Lambda \text { for all nonzero integral ideals } \mathfrak{a}\right\}
$$

To complete the proof of Theorem 1.0.2, we prove the following theorem.

Theorem 7.2.3. Let the notation be as above. Assume that $\chi_{2}=1$. Then the following are equivalent.

1. There exists an element $H \in \mathcal{H}$ such that $C\left(1, H \cdot \mathcal{F}_{0}\right) \in \Lambda^{\times}$.

2. There exists an element $H \in \mathcal{H}$ such that $C(1, H \cdot \mathcal{F})=C_{\lambda}(0, \mathcal{F})$ for all $\mathcal{F} \in M_{\Lambda}$ and for all $\lambda=1, \ldots, h_{F}^{+}$.

3. The homomorphism $\Psi: h / I \rightarrow \Lambda / \widehat{G}_{\chi_{1}}(T)$ is an isomorphism.

4. We have $M_{\Lambda}=M_{\Lambda}^{\prime}$. In particular, we have an isomorphism of $\Lambda$-modules $\operatorname{Hom}_{\Lambda}\left(M_{\Lambda}, \Lambda\right) \simeq \mathcal{H}$.

Proof. We are going to show that (1)-(3) are equivalent and (3)-(4) are equivalent.

First, we prove $(1) \Rightarrow(2)$. Let $H \in \mathcal{H}$ satisfy $C\left(1, H \cdot \mathcal{F}_{0}\right) \in \Lambda^{\times}$. We define

$$
H_{0}=\frac{u}{C\left(1, H \cdot \mathcal{F}_{0}\right)}\left(H-\sum_{i=1}^{m} C\left(1, H \cdot \mathcal{F}_{i}\right) H_{i}\right) \in \mathcal{H} .
$$


We claim that $C\left(1, H_{0} \cdot F\right)=C_{\lambda}(0, F)$ for all $F \in M_{\Lambda}$ and $\lambda=1, \ldots, h_{F}^{+}$. Since the set $\left\{\mathcal{F}_{0}, \ldots, \mathcal{F}_{m}\right\}$ is a $\Lambda$-basis of $M_{\Lambda}$, it suffices to show that $C\left(1, H_{0} \cdot \mathcal{F}_{j}\right)=0$ for all $j=1, \ldots, m$ and $C\left(1, H_{0} \cdot \mathcal{F}_{0}\right)=$ $C_{\lambda}\left(0, \mathcal{F}_{0}\right)$. The computation is as follows. For $1 \leq j \leq m$, we have

$$
\begin{aligned}
C\left(1, H_{0} \cdot \mathcal{F}_{j}\right) & =\frac{u}{C\left(1, H \cdot \mathcal{F}_{0}\right)}\left(C\left(1, H \cdot \mathcal{F}_{j}\right)-\sum_{i=1}^{m} C\left(1, H \cdot \mathcal{F}_{i}\right) C\left(1, H_{i} \cdot \mathcal{F}_{j}\right)\right) \\
& =\frac{u}{C\left(1, H \cdot \mathcal{F}_{0}\right)}\left(C\left(1, H \cdot \mathcal{F}_{j}\right)-C\left(1, H \cdot \mathcal{F}_{j}\right)\right)=0 .
\end{aligned}
$$

Moreover, we have

$$
\begin{aligned}
C\left(1, H_{0} \cdot \mathcal{F}_{0}\right) & =\frac{u}{C\left(1, H \cdot \mathcal{F}_{0}\right)}\left(C\left(1, H \cdot \mathcal{F}_{0}\right)-\sum_{i=1}^{m} C\left(1, H \cdot \mathcal{F}_{i}\right) C\left(1, H_{i} \cdot \mathcal{F}_{0}\right)\right) \\
& ={ }^{(*)} u=C_{\lambda}\left(0, \mathcal{F}_{0}\right)
\end{aligned}
$$

for all $\lambda=1, \ldots, h_{F}^{+}$. Note that the equality $(*)$ is obtained by Proposition 7.2.1(4) and the last equality is obtained by the discussion at the beginning of this section. Thus the statement (2) holds.

Next, we show that $(2) \Rightarrow(3)$. Let $H \in \mathcal{H}$ satisfy $C(1, H \cdot \mathcal{F})=C_{\lambda}(0, \mathcal{F})$ for some $\lambda=1, \ldots, h_{F}^{+}$ and for all $\mathcal{F} \in M_{\Lambda}$. We define

$$
H_{0}=u^{-1} H \in \mathcal{H}
$$

Then $C\left(1, H_{0} \cdot S\right)=0$ for all $S \in S_{\Lambda}$. Moreover, we have

$$
C\left(1, H_{0} \cdot \mathcal{F}_{0}\right)=u^{-1} C\left(1, H \cdot \mathcal{F}_{0}\right)=u^{-1} C_{\lambda}\left(0, \mathcal{F}_{0}\right)=1
$$

Recall that the $\Lambda$-homomorphism

$$
\Psi: h \longrightarrow \Lambda /\left(\widehat{G}_{\chi_{1}}(T)\right) ; T \mapsto C\left(1, T \cdot \mathcal{F}_{S}\right)
$$

is surjective. Therefore, it suffices to show that $\operatorname{ker} \Psi=I$. It is clear that $I \subset \operatorname{ker} \Psi$. It remains to show ker $\Psi \subset I$. Now given any $T \in \operatorname{ker} \Psi \subset h$, we let $H^{\prime} \in \mathcal{H}$ be any lifting of $T$ via $\mathcal{H} \rightarrow h$. We define

$$
H^{\prime \prime}=H^{\prime}-\frac{C\left(1, H^{\prime} \cdot \mathcal{E}\left(\chi_{1}, \mathbf{1}\right)\right)}{C\left(1, H_{0} \cdot \mathcal{E}\left(\chi_{1}, \mathbf{1}\right)\right)} H_{0}
$$


We claim that $H^{\prime \prime}$ kills $\mathcal{E}\left(\chi_{1}, \mathbf{1}\right)$. To see this, we note that

$$
C\left(1, H^{\prime \prime} \cdot \mathcal{E}\left(\chi_{1}, \mathbf{1}\right)\right)=C\left(1, H^{\prime} \cdot \mathcal{E}\left(\chi_{1}, \mathbf{1}\right)\right)-\frac{C\left(1, H^{\prime} \cdot \mathcal{E}\left(\chi_{1}, \mathbf{1}\right)\right)}{C\left(1, H_{0} \cdot \mathcal{E}\left(\chi_{1}, \mathbf{1}\right)\right)} C\left(1, H_{0} \cdot \mathcal{E}\left(\chi_{1}, \mathbf{1}\right)\right)=0
$$

Since $H^{\prime \prime}$ projects to $T$, we obtain that $T \in I$. Thus the statement (3) holds.

Now we show that $(3) \Rightarrow(1)$. Recall that by Proposition 7.2 .1 , we know that $\widehat{G}_{\chi_{1}}(T) \mathcal{F}_{0}=$ $\mathcal{E}\left(\chi_{1}, \mathbf{1}\right)+\mathcal{F}_{S}$ for some $\mathcal{F}_{S} \in S_{\Lambda}$. Then we have

$$
\mathcal{F}_{S} \equiv \mathcal{E}\left(\chi_{1}, \mathbf{1}\right) \bmod \widehat{G}_{\chi_{1}}(T)
$$

Since $\Psi$ is an isomorphism, there exists $H \in h$ such that $C\left(1, H \cdot \mathcal{F}_{S}\right) \in \Lambda^{\times}$. Then we have $\widehat{G}_{\chi_{1}}(T) H \in I$. Let $\widetilde{H} \in \mathcal{I}$ be a lift of $\widehat{G}_{\chi_{1}}(T) H$. Then we have

$$
\begin{aligned}
C\left(1, \widetilde{H} \cdot \mathcal{F}_{0}\right) & =\frac{1}{\widehat{G}_{\chi_{1}}(T)} C\left(1, \widetilde{H} \cdot \mathcal{E}\left(\chi_{1}, \mathbf{1}\right)+\widetilde{H} \cdot \mathcal{F}_{S}\right) \\
& =\frac{1}{\widehat{G}_{\chi_{1}}(T)} C\left(1, \widetilde{H} \cdot \mathcal{F}_{S}\right)=C\left(1, H \cdot \mathcal{F}_{S}\right) \in \Lambda^{\times} .
\end{aligned}
$$

Thus, the statement (1) holds.

Next, we show that $(3) \Rightarrow(4)$. It is clear that $M_{\Lambda} \subset M_{\Lambda}^{\prime}$. We have to show that $M_{\Lambda}^{\prime} \subset M_{\Lambda}$. Given any $\mathcal{F} \in M_{\Lambda}^{\prime}$, we write $\mathcal{F}=\frac{P}{Q} \mathcal{E}\left(\chi_{1}, \mathbf{1}\right)+\frac{S}{T} f$ for some $f \in S_{\Lambda}$ and $P, Q, S, T \in \Lambda$ with $(P, Q)=1,(S, T)=1$ and $Q, T \neq 0$. To show $\mathcal{F} \in M_{\Lambda}$, it suffices to show that $C_{\lambda}(0, \mathcal{F}) \in \Lambda$ for all $\lambda=1, \ldots, h_{F}^{+}$. Moreover, we know that $C_{\lambda}(0, \mathcal{F})=\frac{P}{Q} C_{\lambda}(0, \mathcal{E}(\chi, \mathbf{1}))=\frac{P}{Q} \widehat{G}_{\chi_{1}}(T)$ for all $\lambda=1, \ldots, h_{F}^{+}$, so it is enough to show that $Q$ divides $\widehat{G}_{\chi_{1}}(T)$. We set $\mathcal{F}^{\prime}:=Q \cdot \frac{S}{T} f=Q \mathcal{F}-P \mathcal{E}\left(\chi_{1}, \mathbf{1}\right) \in S_{\Lambda}$. Then $\mathcal{F}^{\prime}$ has the same eigenvalues as those of $\mathcal{E}\left(\chi_{1}, \mathbf{1}\right)$ modulo $Q$. We obtain a surjective homomorphism of $\Lambda$-modules $h / I \rightarrow P \cdot \Lambda / Q \simeq \Lambda / Q$ defined by $H \mapsto C\left(1, H \cdot \mathcal{F}^{\prime}\right)$. Since the statement (3) holds, we have $\Lambda / \widehat{G}_{\chi_{1}}(T) \simeq h / I \rightarrow \Lambda / Q$, which implies that $Q$ divides $\widehat{G}_{\chi_{1}}(T)$.

Finally, we show that $(4) \Rightarrow(3)$. Since $\mathcal{H} \simeq \operatorname{Hom}_{\Lambda}\left(M_{\Lambda}, \Lambda\right)$, there exist $H_{0}, \ldots, H_{m}$ in $\mathcal{H}$ satisfying $C\left(1, H_{i} \cdot \mathcal{F}_{j}\right)=\delta_{i, j}$ for $i, j=0, \ldots, m$, where $\delta_{i, j}$ is 1 if $i=j$ and is 0 if $i \neq j$. In particular, (1) holds. We have seen that (1) and (3) are equivalent, so we know that $(4) \Rightarrow(3)$. 
If $\chi_{2}$ is not a trivial character, then Theorem 7.2 .3 (4) holds automatically since $C_{\lambda}(0, \mathcal{F})=0$ for all $\mathcal{F} \in M_{\Lambda}$. It follows from the exactness of (7.1) that there exists a cusp form $\mathcal{F}_{S}^{\prime}$ which is congruent to $\mathcal{E}\left(\chi_{1}, \chi_{2}\right)$ modulo $A\left(\chi_{1}, \chi_{2}\right)$. We define the $\Lambda$-module homomorphism $\Psi$ by

$$
\Psi: h / I \longrightarrow \Lambda / A\left(\chi_{1}, \chi_{2}\right) ; T \mapsto C\left(1, T \cdot \mathcal{F}_{S}^{\prime}\right)
$$

Note that in the proof of Theorem 7.2.3, the idea to prove (2) $\Rightarrow(3)$ is to construct $H_{0} \in \mathcal{H}$ such that $C\left(1, H_{0} \cdot \mathcal{F}_{0}\right)=1$, which automatically exists if Theorem 7.2 .3 (4) holds. Thus, by the same argument in Theorem 7.2.3, we again see that $\Psi$ is an isomorphism if $\chi_{2}$ is nontrivial. This completes the proof of Theorem 1.0.2.

\subsection{Proof of Theorem 1.0.1: Part 2}

The goal in this subsection is to compute the congruence module of the second short exact sequence in (1.1). First of all, we have a short exact sequence of flat $\Lambda$-modules

$$
0 \longrightarrow \mathcal{I} \longrightarrow \mathcal{H} \longrightarrow \Lambda \longrightarrow 0
$$

where the surjecive homomorphism $\mathcal{H} \longrightarrow \Lambda$ is defined by $T \mapsto C\left(1, T \cdot \mathcal{E}\left(\chi_{1}, \chi_{2}\right)\right)$. This is split after tensoring with $Q(\Lambda)$ since we have

$$
\mathcal{H} \otimes_{\Lambda} Q(\Lambda)=\operatorname{Hom}_{\Lambda}\left(M_{\Lambda}, Q(\Lambda)\right)=\operatorname{Hom}_{\Lambda}\left(S_{\Lambda}, Q(\Lambda)\right) \oplus \operatorname{Hom}_{\Lambda}(\mathcal{E}, Q(\Lambda))
$$

and $\operatorname{Hom}_{\Lambda}(\mathcal{E}, Q(\Lambda)) \simeq Q(\Lambda)$. Here $\mathcal{E}$ is the space generated by the Eisenstein series $\mathcal{E}\left(\chi_{1}, \chi_{2}\right)$. By Theorem 1.0.2, it suffices to show that the congruence module associated to these data is $h / I$. To see this, we recall the following result in [Ohta4, Lemma 1.1.4].

Lemma 7.3.1. Let $R$ be an integral domain with quotient field $Q(R)$, and let

$$
0 \longrightarrow A \stackrel{i}{\longrightarrow} B \stackrel{p}{\longrightarrow} C \longrightarrow 0 \text {. }
$$


be a short exact sequence of flat $R$-modules. Assume that this short exact sequence splits after tensoring with $Q(R)$ over $R$, i.e., we have

$$
0 \longleftarrow A \otimes_{R} Q(R) \stackrel{t}{\longleftarrow} B \otimes_{R} Q(R) \stackrel{s}{\longleftarrow} C \otimes_{R} Q(R) \longleftarrow 0 .
$$

Then we have an isomorphism of R-modules

$$
\mathcal{C}:=C / p(B \cap s(C)) \simeq t(B) / A .
$$

Let $t: \mathcal{H} \otimes_{\Lambda} Q(\Lambda) \longrightarrow \mathcal{I} \otimes_{\Lambda} Q(\Lambda)$ be the splitting map. It follows from (7.2) that

$$
\mathcal{I} \otimes_{\Lambda} Q(\Lambda) \simeq \operatorname{Hom}_{\Lambda}\left(S_{\Lambda} \otimes Q(\Lambda)\right) \simeq h \otimes_{\Lambda} Q(\Lambda) .
$$

Moreover, the image of $\mathcal{H}$ in $h \otimes_{\Lambda} Q(\Lambda)$ is identified with $h \subset h \otimes_{\Lambda} Q(\Lambda)$, and the image of $\mathcal{I}$ in $h$ is identified with $I$. Thus, by Lemma 7.3.1, we see that the congruence module is $t(\mathcal{H}) / \mathcal{I}=h / I$. This completes the proof. 


\section{REFERENCES}

[B] D. Bump, Automorphic forms and representations, Cambridge Studies in Advanced Mathematics 55, Cambridge University Press, Cambridge, 1997.

[Cas] W. Casselman, On Some Results of Atkin and Lehner, Math. Ann. 201 (1973), 301-314.

[Cas-Fro] J. W. S. Cassels and A. Frohlich, Algebraic Number Theory, New York, Academic Press., 1968.

[Cha] C.-L. Chai, Arithmetic minimal compactification of the Hilbert-Blumenthal moduli space, Appendix to [Wil3], Ann. of Math. 131 (1990), 541-554.

[Col] P. Colmez, Résidu en $\mathrm{s}=1$ des fonctions zeta p-adiques, Invent. Math. 91 (1988), 371-389.

[Dpp] S. Dasgupta, H. Darmon, and R. Pollack, Hilbert modular forms and the Gross-Stark conjecture, Ann of Math. 174 (2011), 439-484.

[DR] P. Deligne and K. A. Ribet, Values of abelian $L$-functions at negative integers over totally real fields, Invent. Math. 59 (1980), 227-286.

[DS] F. Diamond and J. Shurman, A first course in modular forms, Grad. Text. Math. 228, Springer-Verlag, Springer-Verlag, New York 2005.

[Dim1] M. Dimitrov, Compactifications arithmétiques des variétés de Hilbert et forms modulaires de Hilbert pour $\Gamma_{1}(\mathfrak{c}, \mathfrak{n})$, Geometric Aspects of Dwork Theory (A. Adolphson, F. Baldassarri, P. Berthelot, N. Katz and F. Loeser, eds.), Walter de Gruyter, Berlin (2004), 525-551.

[Dim2] M. Dimitrov, On Ihara's lemma for Hilbert modular varieties, Compos. Math. 145 (2009), 1114-1146.

[DT] M. Dimitrov and J. Tilouine, Variétés et forms modulaires de Hilbert arithétiques pour $\Gamma_{1}(\mathfrak{c}, \mathfrak{n})$. Geometric Aspects of Dwork Theory (A. Adolphson, F. Baldassarri, P. Berthelot, N. Katz and F. Loeser, eds.), Walter de Gruyter, Berlin (2004), 553-609.

[E] M. Emerton, The Eisenstein ideal in Hida's ordinary Hecke algebra, Int. Math. Res. Not. 15 (1999), 793-802.

[Gor] E. Z. Goren, Lectures on Hilbert modular varieties and modular forms. CRM Monograph Series, 2001.

[H-P] G Harder and R. Pink, Modular konstruierte unverzweigte abelsche $p$-Erweiterungen von $\mathbb{Q}\left(\zeta_{p}\right)$ und die Struktur ihrer Galoisgruppen, Math. Nachr. 159 (1992), 83-99.

[H1] H. Hida, On $p$-adic Hecke algebras for $\mathrm{GL}_{2}$ over totally real fields, Annals of Math. 128 (1988), 295-384. 
[H2] H. Hida, Elementary theory of $L$-functions and Eisenstein series, London Math. Soc. Stud. Texts, Vol. 26, Cambridge Univ. Press, 1993.

[H3] H. Hida, $p$-adic automorphic forms on Shimura varieties, Springer Monographs in Mathematics, Springer-Verlag, New York, 2004.

[Hsieh] M.-L. Hsieh, Eisenstein congruence on unitary groups and Iwasawa main conjectures for CM fields, J. Amer. Math. Soc. 27-3 (2014), 753-862.

[M] T. Miyake, Modular forms, Springer-Verlag, New York, 1989, translated from the Japannese by Yoshitaka Maeda.

[MW] B. Mazur and A. Wiles, Class fields of abelian extension of $\mathbb{Q}$, Invent. Math. 76 (1984), 179-330.

[Neu] J. Neukirch and N. Schappacher, Algebra number theory, vol. 9, Springer Berlin, 1999.

[Ohta1] M. Ohta, On the $p$-adic Eichler-Shimura isomorphism for $\Lambda$-adic cusps froms, J. Reine Angew. Math. 463 (1995), 49-98.

[Ohta2] M. Ohta, Ordinary $p$-adic étale cohomology groups attached to towers of elliptic modular curves, Compos. Math. 115 (1999), 241-301.

[Ohta3] M. Ohta, Ordinary p-adic étale cohomology groups attached to towers of elliptic modular curves II, Math. Ann. 318 (2000), 557-583.

[Ohta4] M. Ohta, Congruence modules related to Eisenstein series, Ann. Sci. École Norm Sup. (4) 36 (2003), 225-269.

[Oz] T. Ozawa, Constant terms of Eisenstein series over a totally real field, Int. J. Number Theory (2017), 309-324.

[Rap] M. Rapoport, Compactifications de l'espace de modules de Hilbert-Blumenthal, Compos. Math. 36 (1978), 255-335.

[Shi] G. Shimura, The special values of the zeta functions associated with Hilbert modular forms, Duke Math. J. 45 (1978), 637-679.

[Sch] R. Schmidt, Some remarks on local newforms for GL(2), J. Ramanujan Math. Soc. 17 (2002), no. 2, 115-147.

[Sha] R. Sharifi, A reciprocity map and the two-variable $p$-adic $L$-function, Ann. of Math. 173 (2011), 251-300.

[Tate] J. Tate, Number theoretic background, Automorphic forms, representations and L-functions (Proc. Sympos. Pure Math., Oregon State Univ., Corvallis, Ore., 1977), Part 2, 326.

[Ven] K. Ventullo, On the rank one abelian Gross-Stark conjecture, Comment. Math. Helv. 90 (2015), 939-963.

[Jun] J. Wang, On the Jacobi sums mod $P^{n}$, J. Number Theory 39 (1991), 50-64. 
[Wa] L. Washington, Introduction to cyclotomic fields, Second edition, Grad. Texts. Math. 83, Springer-Verlag, New York, 1997.

[Wil1] A. Wiles, On p-adic representations for totally real fields, Ann. of Math. 123 (1986), 407-456.

[Wil2] A. Wiles, On ordinary $\lambda$-adic representations associated to modular forms, Invent. Math. 94 (1988), 539-573.

[Wil3] A. Wiles, The Iwasawa conjecture for totally real fields, Ann. of Math 131 (1990), 493-540. 